Supplementary Information

\title{
Tandem Photoredox-Chiral Phosphoric Acid Catalyzed Radical-Radical Cross-Coupling for Enantioselective Synthesis of 3-Hydroxyoxindoles
}

\author{
Yang Zhang, Dan Ye, Lei Shen, Kangjiang Liang, and Chengfeng Xia* \\ Key Laboratory of Medicinal Chemistry for Natural Resource, Ministry of Education, \\ Yunnan Provincial Center for Research \& Development of Natural Products, School \\ of Chemical Science and Technology, Yunnan University, Kunming 650091, China. \\ E-mail: xiacf@ynu.edu.cn
}




\section{Table of Contents}

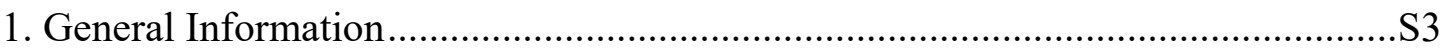

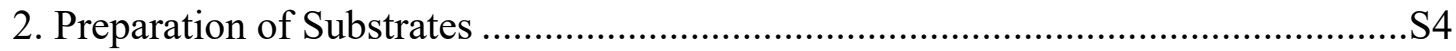

2.1. General Procedures for the Preparation of $\alpha$-ketoesters.................................S4

2.2. General Procedures for the Preparation of Diarylamines...............................S8

3. General Procedures for Enantioselective Synthesis of 3-Hydroxyoxindoles .........S10

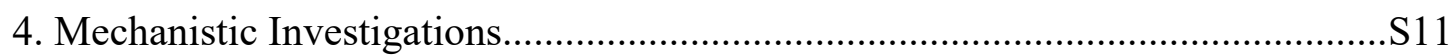

4.1. The Luminescence Quenching Experiments..................................................S11

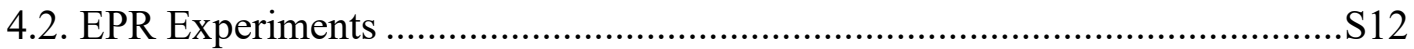

4.3. The Light On-Off Experiment....................................................................... 15

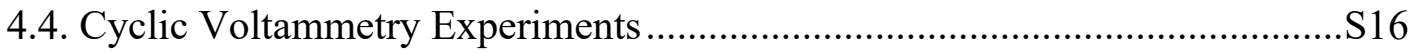

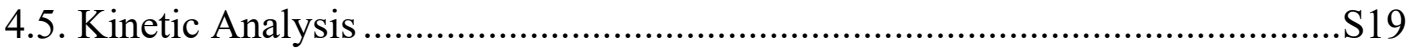

4.6. Controlled Experiments................................................................................ 21

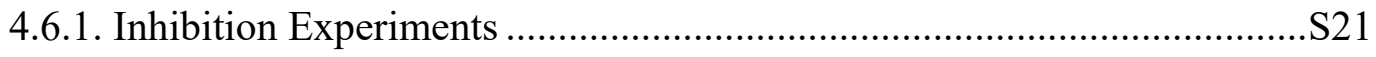

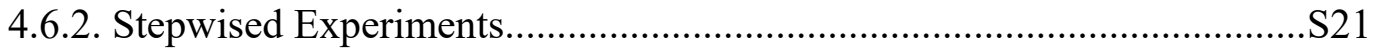

5. Crystal Data and Structure Refinement for Compound 8 ..................................S23

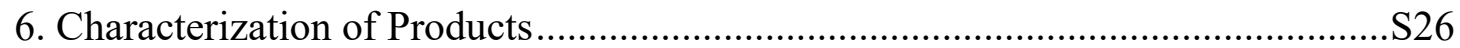

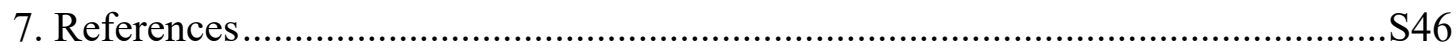

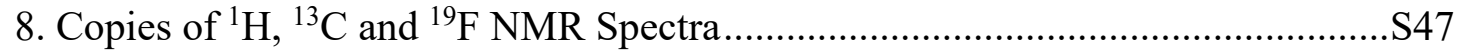

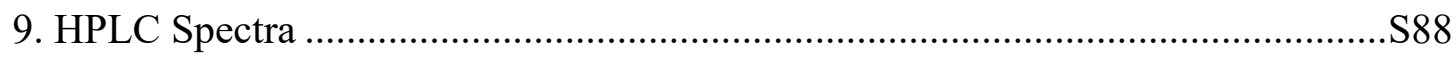




\section{General Information}

All reactions were performed under argon atmosphere using flame-dried glassware unless otherwise noted. DCE was distilled over $\mathrm{CaH}_{2}$ and rigorously degassed by freeze/pump/thaw. Unless otherwise noted, all other reagents and starting materials were purchased from commercial sources and used without further purification. Thin layer chromatography was carried out on GF254 plates ( $0.25 \mathrm{~mm}$ layer thickness). Flash chromatography was performed with 200-300 mesh silica gels. Reactions were monitored by TLC and visualized by a dual short wave/long wave UV lamp. Photochemical reactions were carried with $18 \mathrm{~W}$ blue LED (composed of 18 LED units each with $1.0 \mathrm{~W}) .{ }^{1} \mathrm{H}$ NMR, ${ }^{13} \mathrm{C}$ NMR and ${ }^{19} \mathrm{~F}$ NMR spectra were recorded on Bruker Avance 500 and $600 \mathrm{MHz}$ spectrophotometers. Chemical shifts in ${ }^{1} \mathrm{H}$ NMR spectra were reported in parts per million (ppm) on the $\delta$ scale from an internal standard of residual chloroform (7.26 ppm). Data for ${ }^{1} \mathrm{H}$ NMR were reported as follows: chemical shift, multiplicity $(\mathrm{s}=$ singlet, $\mathrm{d}=$ doublet, $\mathrm{t}=$ triplet, $\mathrm{q}=$ quartet, $\mathrm{m}=$ multiplet or unresolved, br $=$ broad), coupling constant in Herts $(\mathrm{Hz})$ and integration. Data for ${ }^{13} \mathrm{C}$ NMR spectra were reported in terms of chemical shift in ppm from the central peak of $\mathrm{CDCl}_{3}$ (77.16 ppm). High performance liquid chromatography (HPLC) was carried out with Agilent 1260 II Infinity on a UV spectrophotometric detector (Agilent). ESI-MS and HR-ESI-MS were taken on Agilent 6540 Q-TOF spectrometer. Cyclic voltammetry studies was carried out on a CHI $760 \mathrm{E}$ electrochemical workstation (Shanghai $\mathrm{CH}$ Instruments Co., China). The emission spectra were recorded in a Hitachi F-7000 fluorescence spectrometer. Optical rotations were recorded on a JASCO P-2000 polarimeter. EPR spectra were recorded by an ADANI SPINSCAN X spectrometer. 


\section{Preparation of Substrates}

\subsection{General Procedures for the Preparation of $\alpha$-ketoesters}

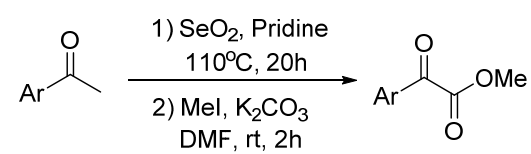

$\alpha$-Ketoesters were prepared according to the known Procedures. ${ }^{[1]}$ To a solution of functionalized acetophenone in pyridine $(1.0 \mathrm{M})$ was added selenium dioxide (2.0 eq.) at room temperature and the mixture was heated to $110{ }^{\circ} \mathrm{C}$ at oil bath for $20 \mathrm{~h}$. After filtration of the reaction mixture, the residue was washed with DCM and the filtrate was concentrated in vacuo. The resulting residue was used for the next reaction without further purification. To a solution of above residue in DMF $(0.6 \mathrm{M})$ was added $\mathrm{K}_{2} \mathrm{CO}_{3}$ (3.0 eq.) and iodomethane (3.0 eq.) at $0{ }^{\circ} \mathrm{C}$ and the mixture was stirred at room temperature for $2 \mathrm{~h}$. After quenching with $1 \mathrm{~N} \mathrm{HCl}$ aq., the resulting mixture was extracted with $\mathrm{Et}_{2} \mathrm{O}$. The combined organic layers were washed with sat. $\mathrm{NaHCO}_{3}$ aq., $10 \% \mathrm{Na}_{2} \mathrm{~S}_{2} \mathrm{O}_{3}$ aq., and brine, then dried over $\mathrm{Na}_{2} \mathrm{SO}_{4}$. After concentration in vacuo, the resulting residue was purified by silica gel chromatography (petroleum ether/ethyl acetate) to give the corresponding $\alpha$-ketoesters.

Methyl 2-(4-iodophenyl)-2-oxoacetate (2b) $)^{[2]}$<smiles>COC(=O)C(=O)c1ccc(I)cc1</smiles>

Following the general procedures, $\mathbf{2 b}$ was isolated as a white powder, $70 \%$ yield; ${ }^{1} \mathrm{H}$ NMR (500 MHz, $\left.\mathrm{CDCl}_{3}\right) \delta 7.89(\mathrm{~d}, J=8.6 \mathrm{~Hz}, 2 \mathrm{H}), 7.75(\mathrm{~d}, J=8.6 \mathrm{~Hz}, 2 \mathrm{H}), 3.98(\mathrm{~s}$, $3 \mathrm{H})$.

\section{Methyl 2-(3-bromo-4-fluorophenyl)-2-oxoacetate (2c)}<smiles>COC(=O)C(=O)c1ccc(F)c(Br)c1</smiles>

Following the general procedures, $2 \mathbf{c}$ was isolated as a yellow powder, 55\% yield; mp: $59.0-61.2{ }^{\circ} \mathrm{C} ;{ }^{1} \mathrm{H}$ NMR $\left(500 \mathrm{MHz}, \mathrm{CDCl}_{3}\right) \delta 8.32(\mathrm{dd}, J=6.6,2.1 \mathrm{~Hz}, 1 \mathrm{H}), 8.05(\mathrm{~m}$, $1 \mathrm{H}), 7.26(\mathrm{t}, J=8.3,1 \mathrm{H}), 3.99(\mathrm{~s}, 3 \mathrm{H}) .{ }^{13} \mathrm{C} \mathrm{NMR}\left(125 \mathrm{MHz}, \mathrm{CDCl}_{3}\right) \delta=182.8,164.2$, 
$162.9,162.1,136.1,131.7,131.6,130.1,117.2,117.0,110.4,110.2,53.1 .{ }^{19} \mathrm{~F}$ NMR $\left(471 \mathrm{MHz}, \mathrm{CDCl}_{3}\right) \delta=-95.5$. HR-ESI-MS calcd. for $\mathrm{C}_{9} \mathrm{H}_{7} \mathrm{BrFO}_{3}[\mathrm{M}+\mathrm{H}]^{+}$: 260.9557, found: 260.9555 .

Methyl 2-(3,5-dichlorophenyl)-2-oxoacetate (2d)<smiles>COC(=O)c1cc(Cl)cc(Cl)c1</smiles>

Following the general procedures, $\mathbf{2 d}$ was isolated as a white powder, $72 \%$ yield; $\mathrm{mp}$ : $54.6-56.9{ }^{\circ} \mathrm{C} ;{ }^{1} \mathrm{H}$ NMR $\left(500 \mathrm{MHz}, \mathrm{CDCl}_{3}\right) \delta=7.93(\mathrm{~d}, J=1.8,2 \mathrm{H}), 7.63(\mathrm{t}, J=1.8$, 1H), $4.00(\mathrm{~s}, 3 \mathrm{H}) .{ }^{13} \mathrm{C} \mathrm{NMR}\left(125 \mathrm{MHz}, \mathrm{CDCl}_{3}\right) \delta=183.0,162.5,136.0,134.9,134.5$, 128.4, 53.2. HR-ESI-MS calcd. for $\mathrm{C}_{9} \mathrm{H}_{7} \mathrm{Cl}_{2} \mathrm{O}_{3}[\mathrm{M}+\mathrm{H}]^{+}$: 232.9767 , found: 232.9769 .

Methyl 2-(3,4-dimethylphenyl)-2-oxoacetate (2e $)^{[3]}$<smiles>COC(=O)C(=O)c1ccc(C)c(C)c1</smiles>

Following the general procedures, $2 \mathrm{e}$ was isolated as a yellow oil, $88 \%$ yield; ${ }^{1} \mathrm{H}$ NMR $\left(600 \mathrm{MHz}, \mathrm{CDCl}_{3}\right) \delta 7.88-7.60(\mathrm{~m}, 2 \mathrm{H}), 7.26(\mathrm{~d}, J=8.0 \mathrm{~Hz}, 1 \mathrm{H}), 3.97(\mathrm{~s}, 3 \mathrm{H}), 2.34$ (s, 3H), $2.32(\mathrm{~s}, 3 \mathrm{H})$.

Methyl 2-oxo-2-(4-propylphenyl)acetate (2f)

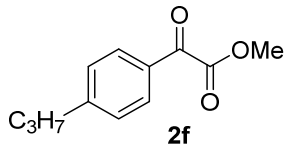

Following the general procedures, $2 \mathbf{f}$ was isolated as a yellow oil, $90 \%$ yield; ${ }^{1} \mathrm{H}$ NMR $\left(500 \mathrm{MHz}, \mathrm{CDCl}_{3}\right) \delta 7.92(\mathrm{~d}, J=8.3,2 \mathrm{H}), 7.30(\mathrm{~d}, J=8.3,2 \mathrm{H}), 3.96(\mathrm{~s}, 3 \mathrm{H}), 2.64(\mathrm{t}, J$ $=7.4,3 \mathrm{H}), 1.61-1.66(\mathrm{~m}, 2 \mathrm{H}), 0.93(\mathrm{t}, J=7.4,3 \mathrm{H}) .{ }^{13} \mathrm{C} \mathrm{NMR}\left(125 \mathrm{MHz}, \mathrm{CDCl}_{3}\right) \delta=$ 185.8, 164.3, 151.0, 130.2, 129.1, 52.7, 38.2, 24.1. HR-ESI-MS calcd. for $\mathrm{C}_{12} \mathrm{H}_{15} \mathrm{O}_{3}[\mathrm{M}$ $+\mathrm{H}]^{+}:$207.1016, found: 207.1014.

Methyl 2-(4-heptylphenyl)-2-oxoacetate (2g)<smiles>CCC(=O)C(=O)c1ccc(C)cc1</smiles> 
Following the general procedures, $2 \mathrm{~g}$ was isolated as a yellow oil, 87\% yield; ${ }^{1} \mathrm{H}$ NMR $\left(600 \mathrm{MHz} \mathrm{CDCl}_{3}\right) \delta 7.93(\mathrm{~d}, J=8.2 \mathrm{~Hz}, 2 \mathrm{H}), 7.31(\mathrm{~d}, J=8.2 \mathrm{~Hz}, 2 \mathrm{H}), 3.98(\mathrm{~s}, 3 \mathrm{H})$, $2.73-2.62(\mathrm{~m}, 2 \mathrm{H}), 1.70-1.57(\mathrm{~m}, 2 \mathrm{H}), 1.38-1.20(\mathrm{~m}, 8 \mathrm{H}), 0.88$ (t, J=7.0 Hz, 3H).

${ }^{13} \mathrm{C} \mathrm{NMR}\left(150 \mathrm{MHz}, \mathrm{CDCl}_{3}\right) \delta=185.7,164.3,151.3,130.3,130.2,129.0,52.6,36.2$, 31.7, 31.0, 29.2, 29.1, 22.6, 14.0. HR-ESI-MS calcd. for $\mathrm{C}_{16} \mathrm{H}_{23} \mathrm{O}_{3}[\mathrm{M}+\mathrm{H}]^{+}$: 263.1642, found: 263.1640 .

Methyl 2-(4-bromo-2-methoxyphenyl)-2-oxoacetate (2h)<smiles>COC(=O)c1ccc(Br)cc1OC</smiles>

Following the general procedures, $\mathbf{2 h}$ was isolated as a brown powder, $83 \%$ yield; $\mathrm{mp}$ : $71.2-73.6{ }^{\circ} \mathrm{C} ;{ }^{1} \mathrm{H}$ NMR $\left(500 \mathrm{MHz}, \mathrm{CDCl}_{3}\right) \delta 7.73(\mathrm{~d}, J=8.3 \mathrm{~Hz}, 1 \mathrm{H}), 7.23(\mathrm{dd}, J=$ 8.3, $1.6 \mathrm{~Hz}, 1 \mathrm{H}), 7.15(\mathrm{~d}, J=1.6 \mathrm{~Hz}, 1 \mathrm{H}), 3.91(\mathrm{~s}, 3 \mathrm{H}), 3.88(\mathrm{~s}, 3 \mathrm{H}) .{ }^{13} \mathrm{C}$ NMR $(125$ $\left.\mathrm{MHz}, \mathrm{CDCl}_{3}\right) \delta=185.4,165.2,160.4,131.9,131.1,124.9,121.8,115.8,56.6,52.5$. HR-ESI-MS calcd. for $\mathrm{C}_{10} \mathrm{H}_{10} \mathrm{BrO}_{4}[\mathrm{M}+\mathrm{H}]^{+}:$272.9757, found: 272.9758 .

Methyl 2-(5-chloro-2-methoxyphenyl)-2-oxoacetate (2i)<smiles>COC(=O)c1cc(Cl)ccc1OC</smiles>

Following the general procedures, $\mathbf{2 i}$ was isolated as a brown powder, $77 \%$ yield; mp: $67.7-69.5{ }^{\circ} \mathrm{C} ;{ }^{1} \mathrm{H}$ NMR $\left(500 \mathrm{MHz}, \mathrm{CDCl}_{3}\right) \delta=7.81(\mathrm{~d}, J=2.3,1 \mathrm{H}), 7.61-7.45(\mathrm{~m}$, $1 \mathrm{H}), 6.94(\mathrm{~d}, J=8.9,1 \mathrm{H}), 3.91(\mathrm{~s}, 3 \mathrm{H}), 3.86(\mathrm{~s}, 3 \mathrm{H}) .{ }^{13} \mathrm{C} \mathrm{NMR}\left(125 \mathrm{MHz}, \mathrm{CDCl}_{3}\right) \delta=$ 185.1, 165.0, 158.8, 135.8, 130.2, 126.9, 124.0, 113.7, 56.7, 52.6. HR-ESI-MS calcd. for $\mathrm{C}_{10} \mathrm{H}_{10} \mathrm{ClO}_{4}[\mathrm{M}+\mathrm{H}]^{+}:$229.0262, found: 229.0266 .

\section{Methyl 2-(2-fluoro-4-methoxyphenyl)-2-oxoacetate $(2 \mathbf{j})^{[4]}$}

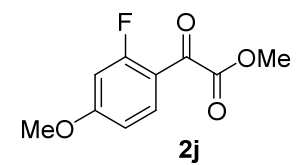

Following the general procedures, $2 \mathbf{j}$ was isolated as a white powder, $65 \%$ yield; ${ }^{1} \mathrm{H}$ NMR (500 MHz, $\left.\mathrm{CDCl}_{3}\right) \delta 7.90(\mathrm{t}, J=8.6 \mathrm{~Hz}, 1 \mathrm{H}), 6.82(\mathrm{dd}, J=8.9,2.3 \mathrm{~Hz}, 1 \mathrm{H}), 6.63$ 
(dd, $J=12.8,2.3 \mathrm{~Hz}, 1 \mathrm{H}), 3.95(\mathrm{~s}, 3 \mathrm{H}), 3.89(\mathrm{~s}, 3 \mathrm{H})$.

\section{Methyl 4-(2-methoxy-2-oxoacetyl)benzoate (2k $)^{[5]}$}

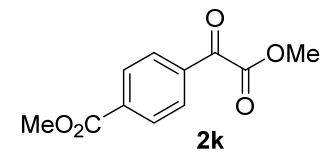

Following the general procedures, $\mathbf{2 k}$ was isolated as a yellow powder, $75 \%$ yield; ${ }^{1} \mathrm{H}$ NMR $\left(500 \mathrm{MHz}, \mathrm{CDCl}_{3}\right) \delta 8.17(\mathrm{~d}, J=8.4,2 \mathrm{H}), 8.10(\mathrm{~d}, J=8.4,2 \mathrm{H}), 4.00(\mathrm{~s}, 3 \mathrm{H})$, $3.97(\mathrm{~s}, 3 \mathrm{H})$.

\section{Methyl 3-(2-methoxy-2-oxoacetyl)benzoate (2l)}

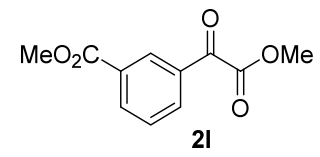

Following the general procedures, $2 \mathbf{I}$ was isolated as a yellow powder, $69 \%$ yield; mp: $57.4-58.9^{\circ} \mathrm{C} ;{ }^{1} \mathrm{H}$ NMR $\left(500 \mathrm{MHz}, \mathrm{CDCl}_{3}\right) \delta=8.65(\mathrm{~s}, 1 \mathrm{H}), 8.32(\mathrm{~d}, J=7.7,1 \mathrm{H}), 8.22$ $(\mathrm{d}, J=7.8,1 \mathrm{H}), 7.61(\mathrm{t}, J=7.8,1 \mathrm{H}), 4.00(\mathrm{~s}, 3 \mathrm{H}), 3.95(\mathrm{~s}, 3 \mathrm{H}) .{ }^{13} \mathrm{C} \mathrm{NMR}(125 \mathrm{MHz}$, $\left.\mathrm{CDCl}_{3}\right) \delta=185.0,165.8,163.4,135.6,134.0,132.8,131.2,131.1,129.2,53.0,52.5$. HR-ESI-MS calcd. for $\mathrm{C}_{11} \mathrm{H}_{11} \mathrm{O}_{5}[\mathrm{M}+\mathrm{H}]^{+}:$223.0601, found: 223.0603 .

\section{Methyl 2-(2-methoxy-2-oxoacetyl)benzoate (2m) ${ }^{[6]}$

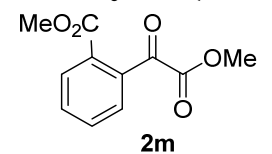

Following the general procedures, $\mathbf{2 m}$ was isolated as a white powder, $70 \%$ yield; ${ }^{1} \mathrm{H}$ NMR $\left(600 \mathrm{MHz}, \mathrm{CDCl}_{3}\right) \delta 8.03(\mathrm{dd}, J=7.7,1.0 \mathrm{~Hz}, 1 \mathrm{H}), 7.68(\mathrm{dd}, J=7.4,1.2 \mathrm{~Hz}$, 1H), $7.64-7.61(\mathrm{~m}, 1 \mathrm{H}), 7.56(\mathrm{dd}, J=7.5,1.1 \mathrm{~Hz}, 1 \mathrm{H}), 3.90(\mathrm{~s}, 3 \mathrm{H}), 3.89$ (s, 3H).

\section{Methyl 2-(9H-fluoren-2-yl)-2-oxoacetate (2n)}

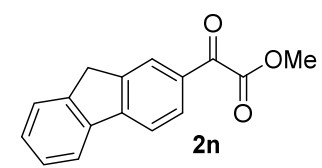

Following the general procedures, $\mathbf{2 n}$ was isolated as a yellow solid, $80 \%$ yield, mp: $104.4-106.5{ }^{\circ} \mathrm{C} ;{ }^{1} \mathrm{H}$ NMR $\left(500 \mathrm{MHz}, \mathrm{CDCl}_{3}\right) \delta=8.17(\mathrm{~s}, 1 \mathrm{H}), 8.05(\mathrm{dd}, J=8.0,0.6$, $1 \mathrm{H}), 7.86(\mathrm{~d}, J=7.9,2 \mathrm{H}), 7.59(\mathrm{~d}, J=6.4,1 \mathrm{H}), 7.48-7.37(\mathrm{~m}, 2 \mathrm{H}), 4.01(\mathrm{~s}, 3 \mathrm{H}), 3.95$ 
(s, 2H). $\left.{ }^{13} \mathrm{C} \mathrm{NMR} \mathrm{(125} \mathrm{MHz,} \mathrm{CDCl}_{3}\right) \delta=185.9,164.5,148.5,144.9,143.5,140.1$, 130.7, 129.7, 128.7, 127.3, 126.6, 125.4, 121.3, 120.1, 52.8, 36.8. HR-ESI-MS calcd. for $\mathrm{C}_{16} \mathrm{H}_{13} \mathrm{O}_{3}[\mathrm{M}+\mathrm{H}]^{+}: 253.0859$, found: 253.0854 .

Methyl 2-oxo-2-(phenanthren-2-yl)acetate (2o)

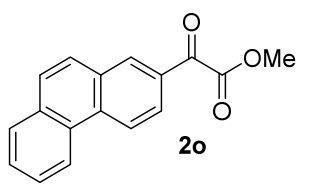

Following the general procedures, 20 was isolated as a yellow solid, 73\% yield, mp: $124.5-126.2{ }^{\circ} \mathrm{C} ;{ }^{1} \mathrm{H}$ NMR $\left(500 \mathrm{MHz}, \mathrm{CDCl}_{3}\right) \delta=8.74(\mathrm{~d}, J=8.7,1 \mathrm{H}), 8.68(\mathrm{~d}, J=$ 8.6, 1H), $8.57(\mathrm{~d}, J=1.0,1 \mathrm{H}), 8.23(\mathrm{dd}, J=8.7,1.2,1 \mathrm{H}), 7.91(\mathrm{dd}, J=6.0,3.0,1 \mathrm{H})$, $7.80(\mathrm{~s}, 2 \mathrm{H}), 7.75-7.64(\mathrm{~m}, 2 \mathrm{H}), 4.06(\mathrm{~s}, 3 \mathrm{H}) .{ }^{13} \mathrm{C} \mathrm{NMR}\left(125 \mathrm{MHz}, \mathrm{CDCl}_{3}\right) \delta=185.7$, 164.1, 134.7, 133.4, 132.6, 131.4, 130.2, 129.5, 128.8, 128.4, 127.3, 125.8, 123.58, 123.56, 52.9. HR-ESI-MS calcd. for $\mathrm{C}_{17} \mathrm{H}_{13} \mathrm{O}_{3}[\mathrm{M}+\mathrm{H}]^{+}: 265.0859$, found: 265.0858 .

\section{Methyl 2-oxo-2-(5,6,7,8-tetrahydronaphthalen-2-yl)acetate (2p)}

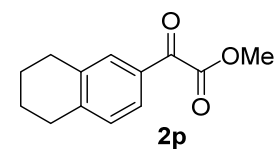

Following the general procedures, $2 \mathbf{p}$ was isolated as a yellow oil, $85 \%$ yield; ${ }^{1} \mathrm{H}$ NMR $\left(500 \mathrm{MHz} \mathrm{CDCl}_{3}\right) \delta=7.70(\mathrm{~d}, J=8.0,2 \mathrm{H}), 7.18(\mathrm{~d}, J=7.8,1 \mathrm{H}), 3.96(\mathrm{~s}, 3 \mathrm{H}), 2.82(\mathrm{~d}$, $J=6.1,4 \mathrm{H}), 1.82(\mathrm{t}, J=3.1,4 \mathrm{H}) .{ }^{13} \mathrm{C} \mathrm{NMR}\left(125 \mathrm{MHz}, \mathrm{CDCl}_{3}\right) \delta=185.4,165.2,160.4$, 131.9, 131.1, 124.9, 121.8, 115.8, 56.6, 52.5. HR-ESI-MS calcd. for $\mathrm{C}_{13} \mathrm{H}_{15} \mathrm{O}_{3}[\mathrm{M}+$ $\mathrm{H}]^{+}:$219.1016, found:219.1011.

\subsection{General Procedures for the Preparation of Diarylamines ${ }^{[7]}$}

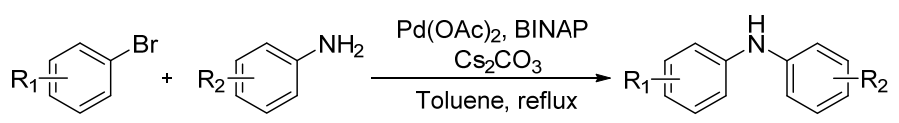

To a nitrogen flushed round bottom flask was charged with bromobenzene (1.0 eq.), arylamine (1.1 eq.), BINAP (10.0 mol\%), $\mathrm{Pd}(\mathrm{OAc}) 2$ (9.0 mol\%), and $\mathrm{Cs}_{2} \mathrm{CO}_{3}$ (1.6 eq.). Toluene was then added and the mixture was bubbled with nitrogen for $15 \mathrm{~min}$. The mixture was refluxed at oil bath for $24 \mathrm{~h}$ before quenching by water. Then the solution 
was extracted for three times and the combined organic layer was dried with $\mathrm{Na}_{2} \mathrm{SO}_{4}$ for two hours. After removal of solvent, the crude product was purified by column chromatography on silica gel.

\section{4-Methoxy- $N$-(4-methoxyphenyl)-2,6-dimethylaniline (1b)}

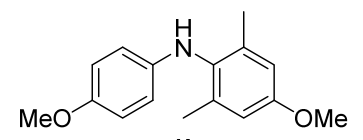

$1 \mathrm{~b}$

Following the general procedures, $\mathbf{1 b}$ was isolated as a white powder, $40 \%$ yield; $\mathrm{mp}$ : $104.5-106.6{ }^{\circ} \mathrm{C} ;{ }^{1} \mathrm{H}$ NMR $(600 \mathrm{MHz}, \mathrm{DMSO}) \delta=6.96(\mathrm{~d}, J=7.7,2 \mathrm{H}), 6.83(\mathrm{~d}, J=$ 8.8, 2H), $6.58(\mathrm{~s}, 2 \mathrm{H}), 3.69$ (s, 3H), 3.57 (s, 3H), $2.13(\mathrm{~s}, 6 \mathrm{H}) .{ }^{13} \mathrm{C}$ NMR (150 MHz, DMSO) $\delta=153.8,149.9141 .1,137.6,130.9,120.0,116.2,115.0,59.9,55.7,16.5$. HRESI-MS calcd. for $\mathrm{C}_{16} \mathrm{H}_{20} \mathrm{NO}_{2}[\mathrm{M}+\mathrm{H}]^{+}:$258.1489, found: 258.1484 .

\section{$N$-(4-methoxyphenyl)-2,4,6-trimethylaniline (1c) $)^{[8]}$}

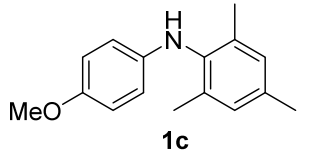

Following the general procedures, 1c was isolated as a brown powder, $45 \%$ yield; ${ }^{1} \mathrm{H}$ NMR (600 MHz, DMSO) $\delta=6.90(\mathrm{~s}, 2 \mathrm{H}), 6.70(\mathrm{~d}, J=8.7,2 \mathrm{H}), 6.46-6.32(\mathrm{~m}, 2 \mathrm{H})$, $3.64(\mathrm{~s}, 3 \mathrm{H}), 2.25(\mathrm{~s}, 3 \mathrm{H}), 2.13(\mathrm{~s}, 6 \mathrm{H})$. 


\section{General Procedures for Enantioselective Synthesis of 3-Hydroxyoxindoles}

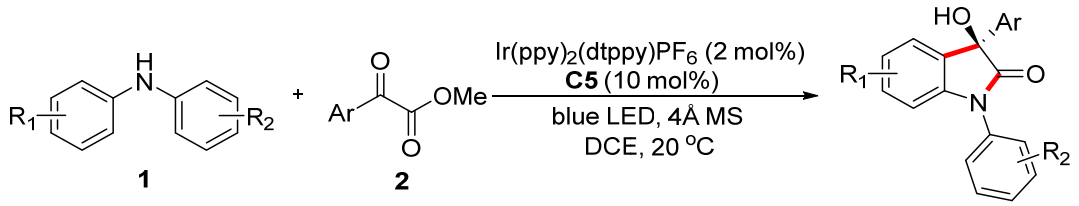

To a $10 \mathrm{~mL}$ flame-dry Schlenk reaction tube equipped with a magnetic stir bar, diarylamine 1 (0.10 mmol), $\alpha$-ketoester 2 (0.12 mmol), Ir(ppy)2(dtbbpy)PF6 (0.0020 mmol), C5 (0.010 mmol), $4 \AA \mathrm{MS}(25.0 \mathrm{mg})$, and dry DCE $(1.0 \mathrm{~mL})$ were sequentially added. The reaction mixture was degassed three times by freeze-pump-thaw method. The tube was placed approximately $10 \mathrm{~cm}$ away from a $18 \mathrm{~W}$ blue light emitting diode (LED) lamp (Ouying-5313, $18 \mathrm{~W}$ LED, $80 \mathrm{~cd} / \mathrm{w}$ ), and the reaction mixture was stirred at $20^{\circ} \mathrm{C}$ under an argon atmosphere. After the reaction was complete (by TLC analysis), the reaction mixture was directly loaded onto a short silica gel column. The crude was chromatographed by gradient elution (petroleum ether/ethyl acetate) to afford desired products.
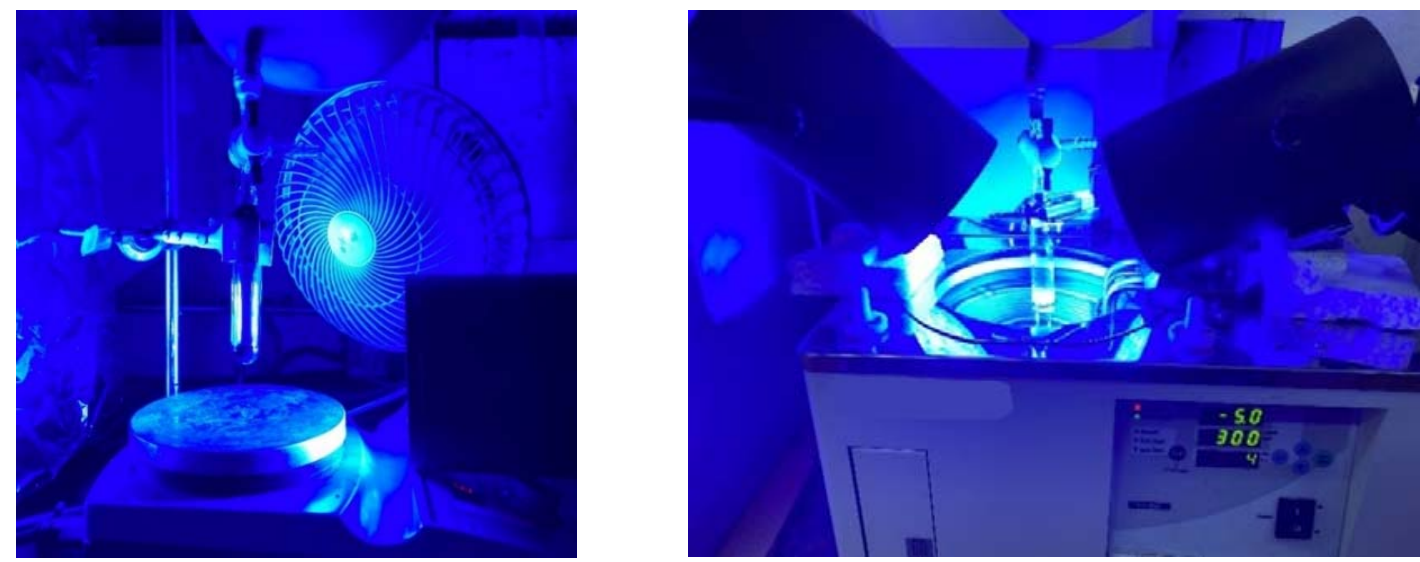

Figure S1. Details for the photochemical reaction setup at room temperature (left) and $-5{ }^{\circ} \mathrm{C}$ (right).

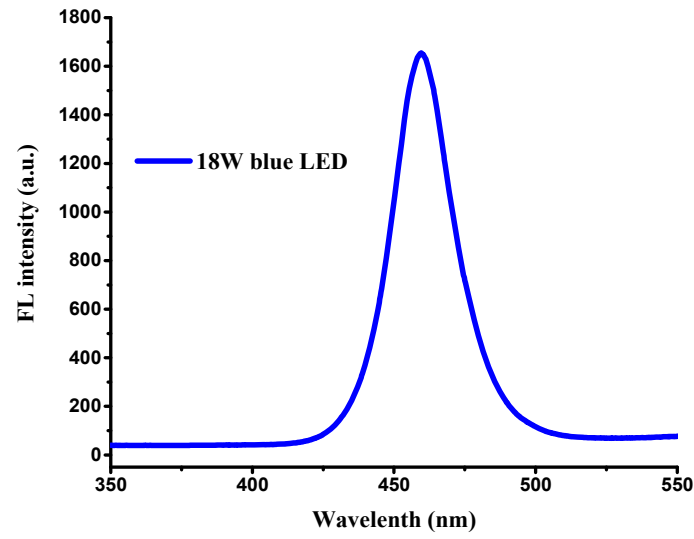

Figure S2. Emission spectra of the $18 \mathrm{~W}$ blue LED lamp (maximum emission at $\lambda=460 \mathrm{~nm}$ ). 


\section{Mechanistic Investigations}

\subsection{The Luminescence Quenching Experiments}

Fluorescence quenching experiments were performed on a Hitachi F-7000 fluorescence spectrometer. All $\operatorname{Ir}(\mathrm{ppy})_{2}(\mathrm{dtbbpy}) \mathrm{PF}_{6}$ solutions were excited at $450 \mathrm{~nm}$ and the emission intensity was collected from 475 to $675 \mathrm{~nm}$. In a typical experiment, to a $5 \times 10^{-5} \mathrm{M}$ solution of $\operatorname{Ir}(\text { ppy })_{2}(\mathrm{dtbbpy}) \mathrm{PF}_{6}$ in DCE (rigorously degassed by freeze/pump/thaw) was added the appropriate amount of quencher in a $10 \times 10 \mathrm{~mm}$ light path quartz fluorescence cuvette. After degassing the sample with a stream of argon for 10 minutes, the emission of the sample was collected.

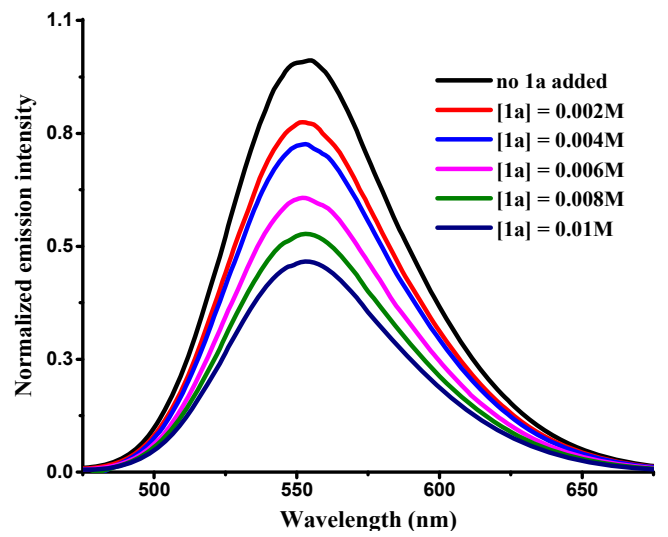

Figure S3. Quenching of the $\operatorname{Ir}(\mathrm{ppy})_{2}(\mathrm{dtbbpy}) \mathrm{PF}_{6}$ emission $\left(5 \times 10^{-5} \mathrm{M}\right.$ in DCE $)$ in the presence of increasing amounts of $\mathbf{1 a}$.

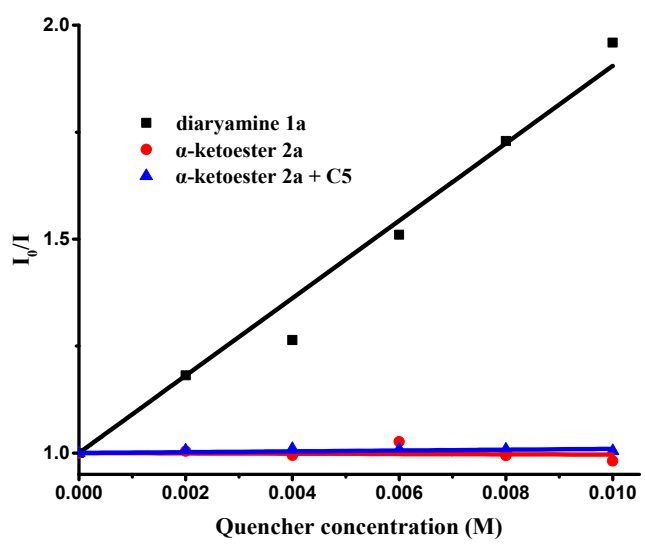

Figure S4. $\operatorname{Ir}(\mathrm{ppy})_{2}(\mathrm{dtbbpy}) \mathrm{PF}_{6}$ emission quenching with ketoester 2a, ketoester 2a in the presence of phosphoric acid C5 (10 mol\%) and diarylamine $\mathbf{1 a}$. 


\subsection{EPR Experiments}

EPR spectra were recorded at room temperature on an ANANI SPINSCAN $\mathrm{X}$ spectrometer.

EPR spectrometer was operated at $9.446598 \mathrm{GHz}$. Typical spectrometer parameters are shown as following: scan range: $15.00 \mathrm{mT}$; center field set: $336.00 \mathrm{mT}$; time constant: $0.046 \mathrm{~s}$; scan time: $60.0 \mathrm{~s}$; modulation amplitude: $100 \mu \mathrm{T}$; modulation frequency: $93750 \mathrm{~Hz}$; power attenuation: $10 \mathrm{~dB}$. A mixture of 4,4'dimethoxydiphenylamine 1a $(0.10 \mathrm{mmol}), \operatorname{Ir}(\mathrm{ppy})_{2}(\mathrm{dtbbpy}) \mathrm{PF}_{6}(0.0020 \mathrm{mmol}), \mathrm{PBN}$ $(0.20 \mathrm{mmol})$ in degassed dry DCE $(1.0 \mathrm{~mL})$ was stirred under an argon atmosphere with irradiation by a commercially available blue LED for $15 \mathrm{~min}$. Afterwards, $20 \mu \mathrm{L}$ of the mixture was quickly taken out into a small tube and analyzed by EPR.

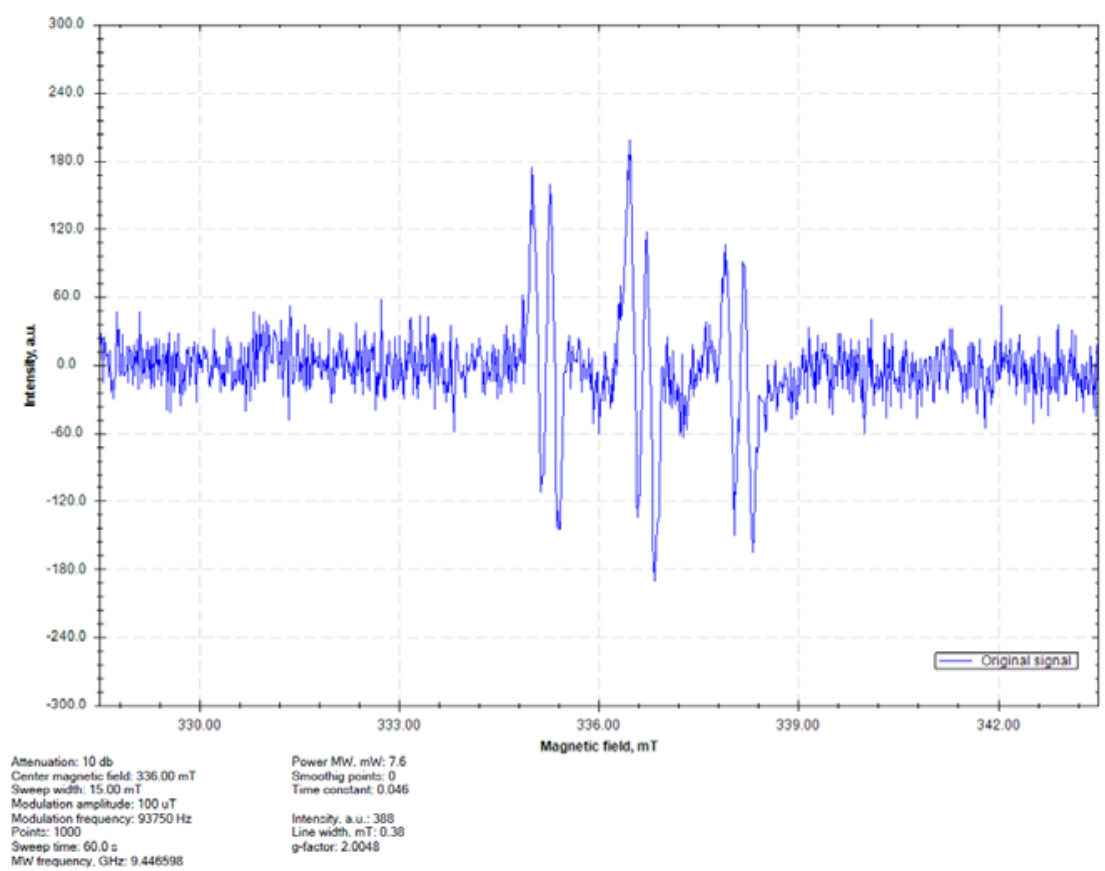

Figure S5. EPR spectra of 1a. 




MS Formula Results: + Scan (0.8231 min) Sub (XZD-PBN-3.d)

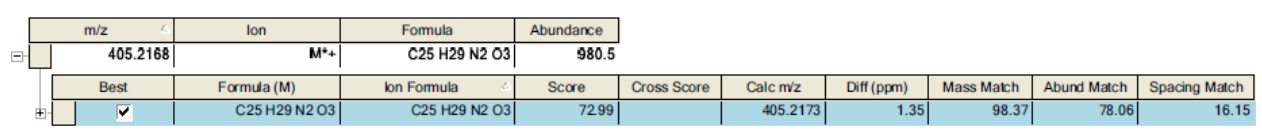

Figure S6. HR-MS spectra of 39.

A mixture of methyl benzoylformate $2 \mathrm{a}(0.10 \mathrm{mmol}), \operatorname{Ir}(\mathrm{ppy})_{2}(\mathrm{dtbbpy}) \mathrm{PF}_{6}(0.0020$ mmol), and PBN (0.20 mmol) in degassed dry DCE $(1.0 \mathrm{~mL})$ was stirred under an argon atmosphere with irradiation by a commercially available blue LED for $15 \mathrm{~min}$. Afterwards, $20 \mu \mathrm{L}$ of the mixture was quickly taken out into a small tube and analyzed by EPR.

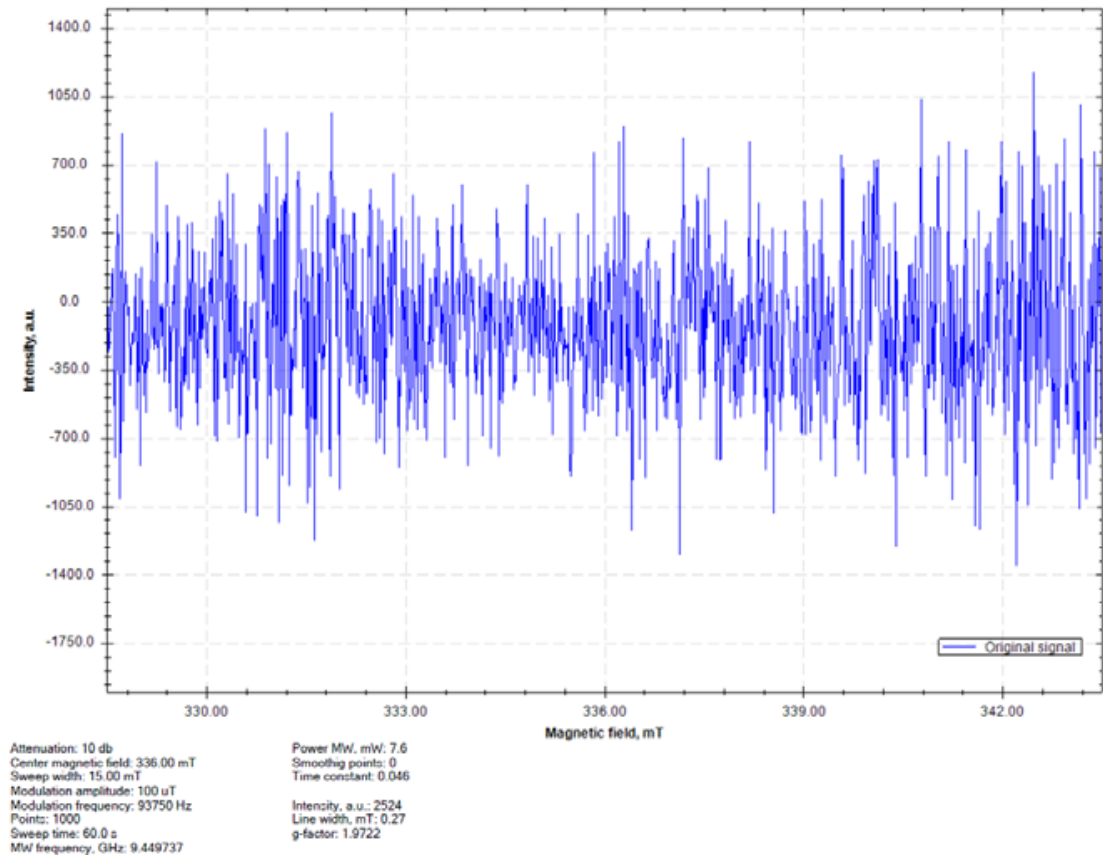

Figure S7. EPR spectra of 2 a. 
A mixture of methyl benzoylformate 2a $(0.10 \mathrm{mmol}), \operatorname{Ir}(\mathrm{ppy})_{2}(\mathrm{dtbbpy}) \mathrm{PF}_{6}$ (0.0020 mmol), C5 (0.010 mmol), and PBN (0.20 mmol) in degased dry DCE (1.0 $\mathrm{mL}$ ) was stirred under an argon atmosphere with irradiation by a commercially available blue LED for 15 min. Afterwards, $20 \mu \mathrm{L}$ of the mixture was quickly taken out into a small tube and analyzed by EPR.

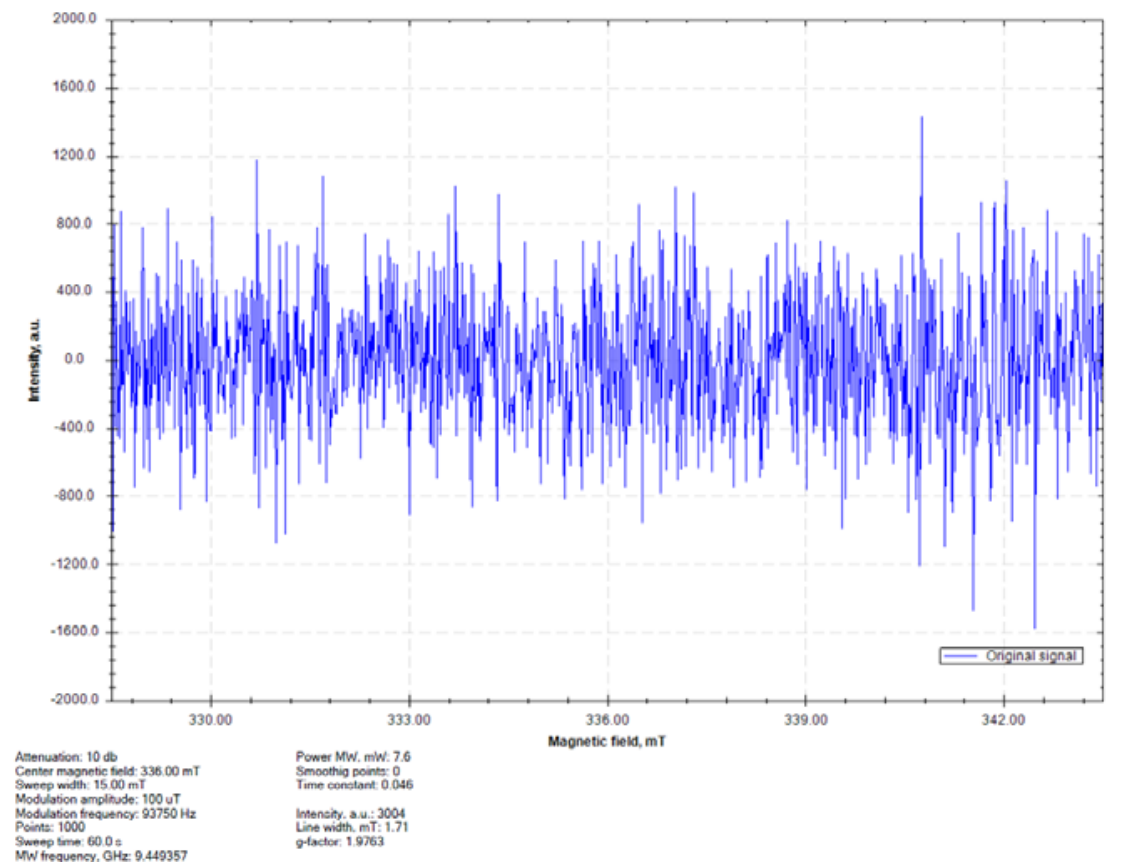

Figure S8. EPR spectra of 2a in the presence of C5. 


\subsection{The Light On-Off Experiment}

Following the standard procedures, the reaction between 4,4'dimethoxydiphenylamine $1 \mathbf{a}(0.10 \mathrm{mmol})$ and methyl benzoylformate $2 \mathbf{a}(0.12 \mathrm{mmol})$ was conducted for light-dark experiment. Aliquots of samples were taken out at various time points during the reaction. The crude NMR was taken on the concentrated crude reaction mixture and calculated using diphenylacetonitrile as an internal standard.

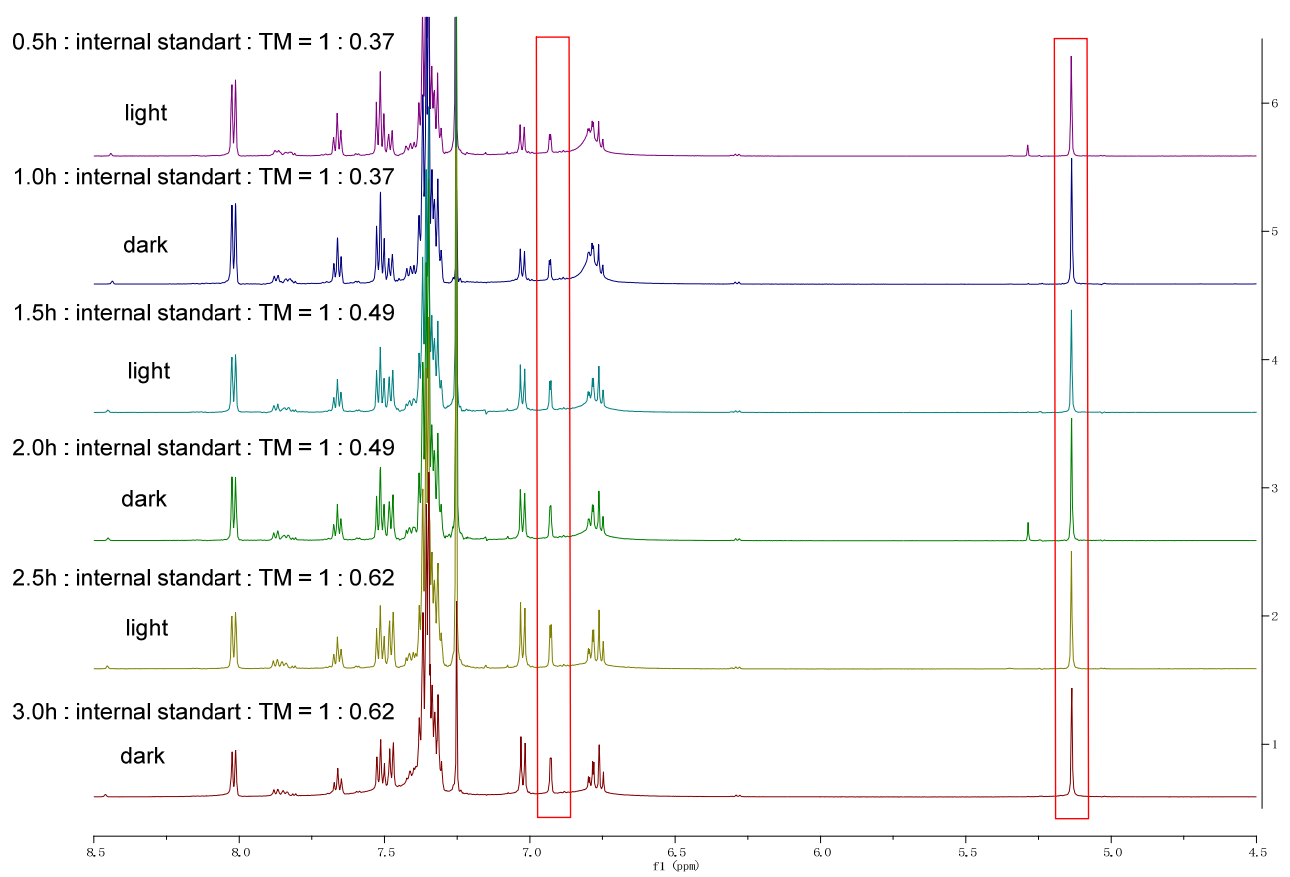

Figure S9. The light on-off experiment (NMR Traces). 


\subsection{Cyclic Voltammetry Experiments}

Tetrabutylammonium hexafluorophosphate (1.0 mmol) and 4,4'dimethoxydiphenylamine 1a $(0.10 \mathrm{mmol})$ were dissolved in dry DCE $(10 \mathrm{~mL})$ and the solution was vigorously bubbled with $\mathrm{N}_{2}$ for 5 minutes prior to the measurement. The oxidation potential was measured using a glassy carbon working electrode, a platinum wire counter electrode, and a saturated calomel electrode (SCE) at $0.1 \mathrm{~V} / \mathrm{s}$ scan rate.

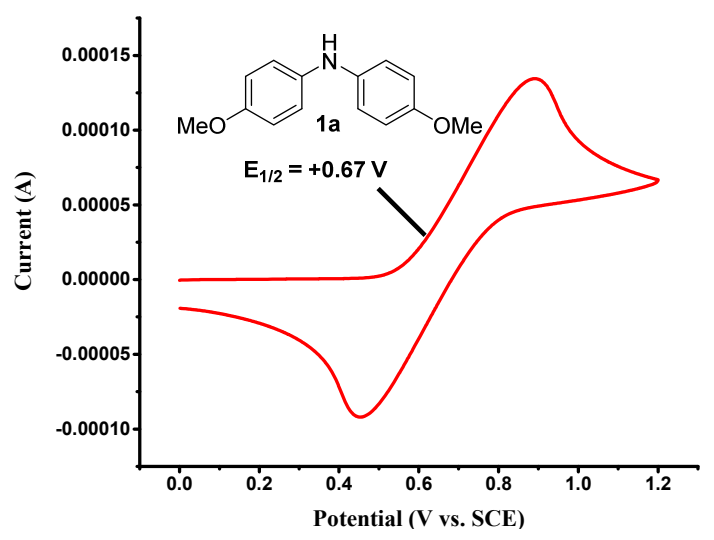

Figure S10. Cyclic voltammogram of substrate 1a in DCE.

Tetrabutylammonium hexafluorophosphate $(1.0 \mathrm{mmol})$ and methyl benzoylformate 2a $(0.10 \mathrm{mmol})$ were dissolved in dry DCE $(10 \mathrm{~mL})$ and the solution was vigorously bubbled with $\mathrm{N}_{2}$ for 5 minutes prior to the measurement. The reduction potential was measured using a glassy carbon working electrode, a platinum wire counter electrode, and a saturated calomel electrode (SCE) at $0.1 \mathrm{~V} / \mathrm{s}$ scan rate.

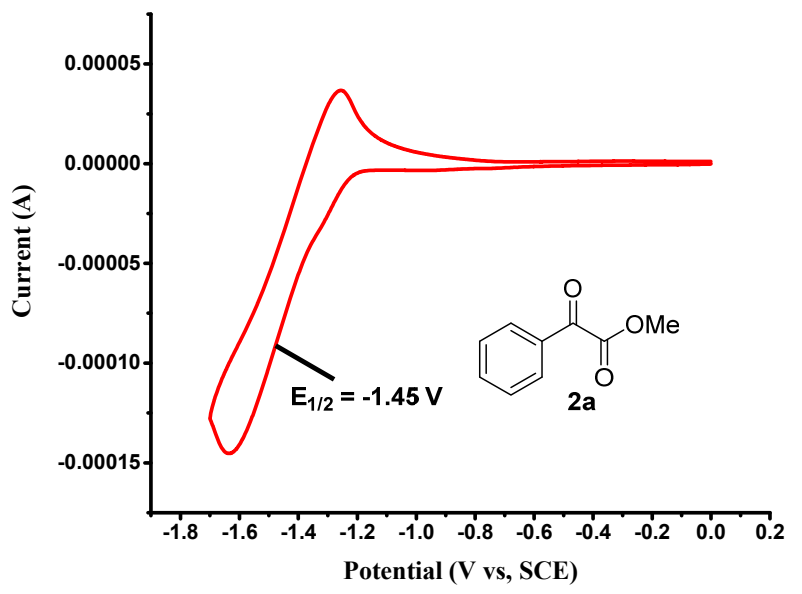

Figure S11. Cyclic voltammogram of substrate $\mathbf{2 a}$ in DCE 
Tetrabutylammonium hexafluorophosphate $(1.0 \mathrm{mmol})$, methyl benzoylformate $2 \mathrm{a}$ $(0.10 \mathrm{mmol})$, and phosphoric acid C5 $(0.10 \mathrm{mmol})$ were dissolved in dry DCE $(10 \mathrm{~mL})$ and the solution was vigorously bubbled with $\mathrm{N}_{2}$ for 5 minutes prior to the measurement. The reduction potential was measured using a glassy carbon working electrode, a platinum wire counter electrode, and a saturated calomel electrode (SCE) at $0.1 \mathrm{~V} / \mathrm{s}$ scan rate.

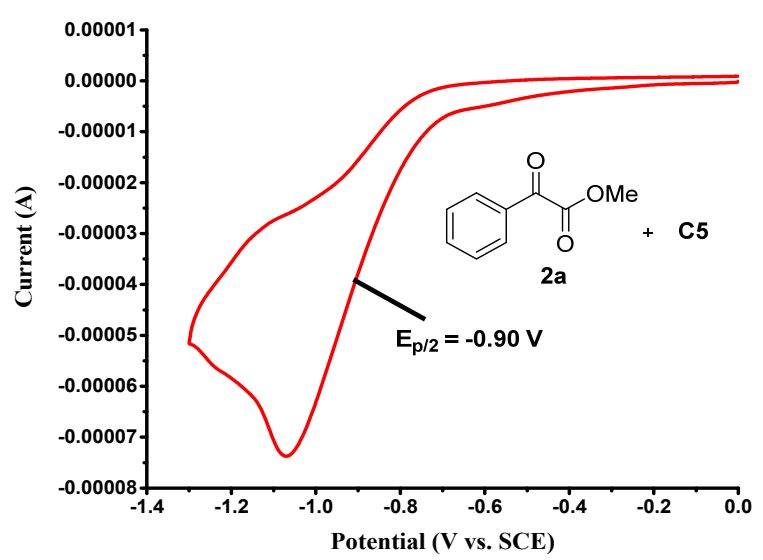

Figure S12. Cyclic voltammogram of substrate 2a with C5 in DCE.

The zero-zero vibrational state excitation energy $E_{0,0}$ was estimated by the corresponding energy of the wavelength at which emission and absorption overlap. This wavelength was determined setting the intensity of emission $\lambda_{\max }$ to the absorbance at excitation wavelength $(508 \mathrm{~nm})$. Excited state oxidation and reduction potentials were calculated by the following approximating formulas:

$$
E_{0,0}=\mathrm{hc} / \lambda_{\max }, E_{1 / 2}\left(\mathrm{M}^{*} / \mathrm{M}^{-}\right)=E_{1 / 2}\left(\mathrm{M} / \mathrm{M}^{-}\right)+E_{0,0} \text { and } E_{1 / 2}\left(\mathrm{PC}^{+} / \mathrm{PC}^{*}\right)=E_{1 / 2}
$$
$\left(\mathrm{PC}^{+} / \mathrm{PC}\right)-E_{0,0}$

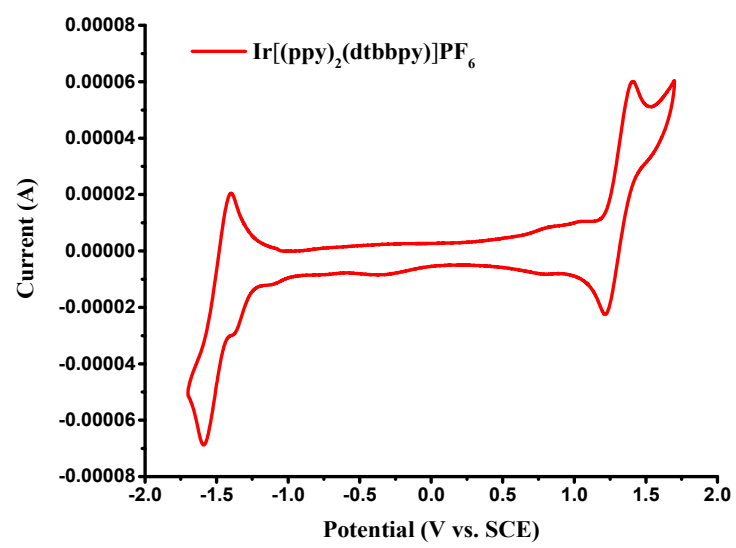

Figure S13. CV scans (scan rate $0.1 \mathrm{~V} / \mathrm{s}$ ) of substrates: $\operatorname{Ir}\left[(\mathrm{ppy})_{2}(\mathrm{dtbbpy})\right] \mathrm{PF}_{6}(3 \mathrm{mM})$ in $0.1 \mathrm{M}$ ${ }^{\mathrm{n}} \mathrm{Bu}_{4} \mathrm{NPF}_{6} / \mathrm{DCE}$. 


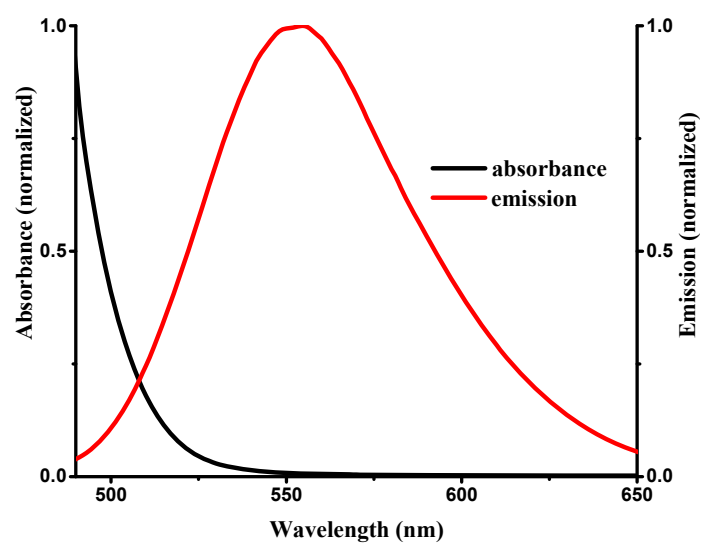

Figure S14. Normalized absorption and emission spectra of $\operatorname{Ir}\left[(\mathrm{ppy})_{2}(\mathrm{dtbbpy})\right] \mathrm{PF}_{6}$ in dry DCE, the intersect wavelength was $508 \mathrm{~nm}$.

Table S1. The redox potential of $\operatorname{Ir}\left[(\text { ppy })_{2}(\mathrm{dtbbpy})\right] \mathrm{PF}_{6}$ in DCE

\begin{tabular}{cccccc}
\hline $\begin{array}{c}{[\mathrm{Ir}]} \\
E_{1 / 2}\left(\mathrm{Ir}^{\mathrm{IV}} / \mathrm{Ir}^{\mathrm{III}}\right) \\
{[\mathrm{v}]}\end{array}$ & $\begin{array}{c}E_{1 / 2}\left(\mathrm{Ir}^{\mathrm{II}}{ }^{*} / \mathrm{Ir}^{\mathrm{II}}\right) \\
{[\mathrm{v}]}\end{array}$ & $\begin{array}{c}E_{1 / 2}\left(\mathrm{Ir}_{\mathrm{IV}} / \mathrm{Ir}_{\mathrm{III}}\right) \\
{[\mathrm{v}]}\end{array}$ & $\begin{array}{c}E_{1 / 2}\left(\mathrm{Ir}^{\mathrm{III}} / / \mathrm{Ir}^{\mathrm{II}}\right) \\
{[\mathrm{v}]}\end{array}$ & $\begin{array}{c}\text { Emission } \\
\lambda \max \\
{[\mathrm{nm}]}\end{array}$ & $\begin{array}{c}E_{0,0} \\
{[\mathrm{ev}]}\end{array}$ \\
\hline-1.13 & +0.94 & +1.31 & -1.50 & 508 & 2.44 \\
\hline
\end{tabular}




\subsection{Kinetic Analysis}

The standard series of product 3 solutions were prepared with concentration of 0.01 , $0.02,0.03,0.04,0.05,0.06,0.07$, and $0.08 \mathrm{mmol} / \mathrm{mL}$. Under the condition of the same injection volume, the peak area was detected separately by HPLC. The linear regression was performed for the concentration using the peak area where $\mathrm{x}$ is the peak area and $\mathrm{y}$ is the concentration $(\mathrm{mmol} / \mathrm{mL})$.

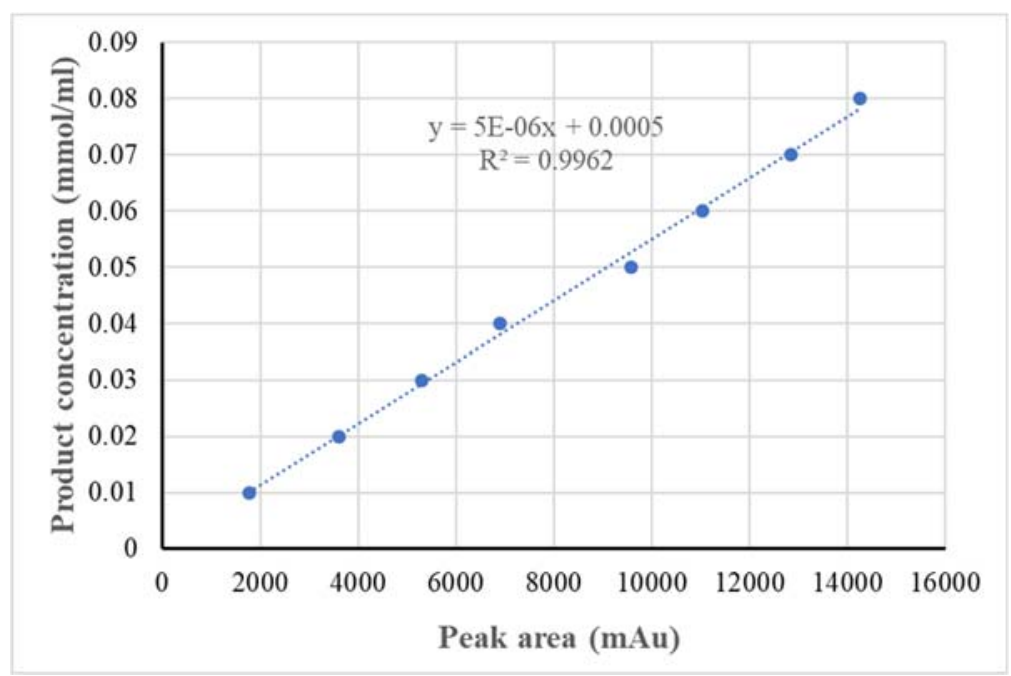

Figure S15. Standard curve of the product 3 .

To a $10 \mathrm{~mL}$ flame-dry Schlenk reaction tubes equipped with a magnetic stir bar, 4,4'dimethoxydiphenylamine 1a $(0.10 \mathrm{mmol})$, methyl benzoylformate $2 \mathbf{2 a}(0.12 \mathrm{mmol})$, $\operatorname{Ir}(\text { ppy })_{2}(\mathrm{dtbbpy}) \mathrm{PF}_{6}(0.0020 \mathrm{mmol}), 4 \AA \mathrm{MS}(25.0 \mathrm{mg})$, dry DCE $(1.0 \mathrm{~mL})$, and 0.010 mmol different chiral phosphoric acid $(\mathbf{C 1}, \mathbf{C 2}, \mathbf{C 3}, \mathbf{C 4}, \mathbf{C 5}, \mathbf{C 6}, \mathbf{C 7})$ were sequentially added. The reaction mixture was degassed three times by freeze-pump-thaw method. The tube was placed approximately $10 \mathrm{~cm}$ away from a $18 \mathrm{~W}$ blue LED lamp, and detect the concentration of the product in the crude reaction mixture by HPLC after different time intervals: 20, 40, 60, 80, 100, 120, 140, 160, 180, 200, and 220 minutes. 


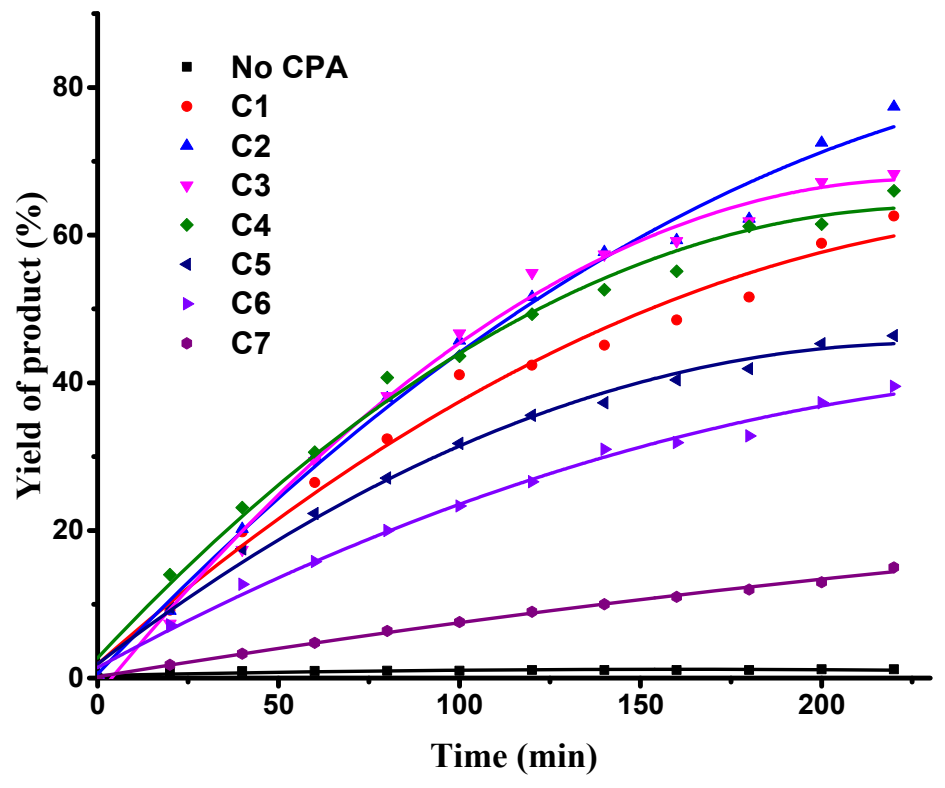

Figure S16. Kinetic analysis of the cross-coupling reaction in the presence of phosphoric acid catalyst. 


\subsection{Controlled Experiments}

\subsubsection{Inhibition Experiments}

Table S2. Verification of the radical pathway by inhibitors.

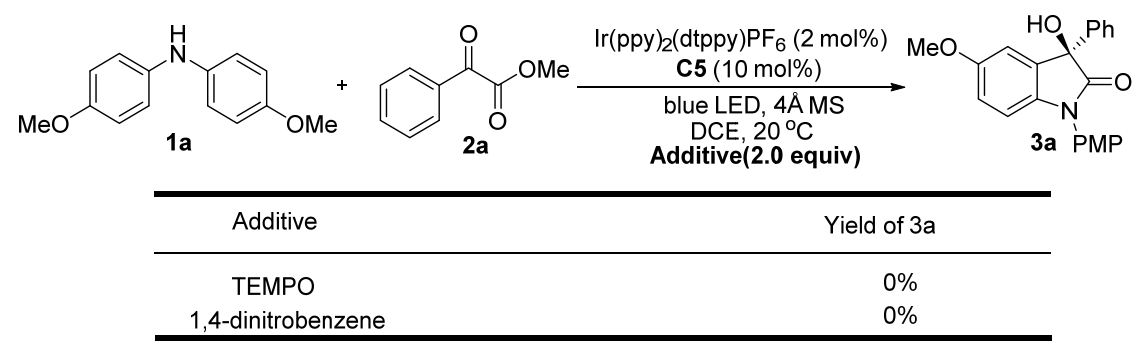

Following the standard procedure, to a $10 \mathrm{~mL}$ flame-dry Schlenk reaction tube equipped with a magnetic stir bar. 4,4'-dimethoxydiphenylamine 1a $(0.10 \mathrm{mmol})$, methyl benzoylformate 2a (0.12 mmol), $\operatorname{Ir}(\mathrm{ppy})_{2}(\mathrm{dtbbpy}) \mathrm{PF}_{6}(0.002 \mathrm{mmol}), \mathbf{C 5}(0.010$ mmol), $4 \AA \mathrm{MS}(25.0 \mathrm{mg})$, additive $(0.20 \mathrm{mmol})$ and dry DCE $(1.0 \mathrm{~mL})$ were sequentially added. The reaction mixture was degassed three times by freeze-pumpthaw method. The tube was placed approximately $10 \mathrm{~cm}$ away from a $18 \mathrm{~W}$ blue LED lamp, and the reaction mixture was stirred at $20^{\circ} \mathrm{C}$ under an argon atmosphere for 24 h.

\subsubsection{Stepwised Experiments}

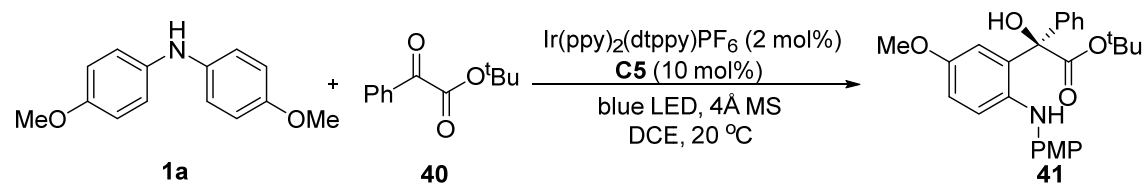

Following the standard procedure, to a $10 \mathrm{~mL}$ flame-dry Schlenk reaction tube equipped with a magnetic stir bar. 4,4'-dimethoxydiphenylamine 1a $(0.10 \mathrm{mmol})$, tertbutyl 2-oxo-2-phenylacetate 40 (0.12 mmol), $\operatorname{Ir}($ ppy)2(dtbbpy)PF 6 (0.0020 mmol), C5 (0.010 mmol), $4 \AA \mathrm{MS}(25.0 \mathrm{mg})$, and dry DCE $(1.0 \mathrm{~mL})$ were sequentially added. The reaction mixture was degassed three times by freeze-pump-thaw method. The tube was placed approximately $10 \mathrm{~cm}$ away from a $18 \mathrm{~W}$ blue LED lamp, and the reaction mixture was stirred at $20{ }^{\circ} \mathrm{C}$ under an argon atmosphere. After the reaction was complete (by TLC analysis), the reaction mixture was directly loaded onto a short silica gel column, the crude product was subjected to column chromatography (petroleum 
ether/ethyl acetate $=5 / 1$ ) on silica gel. Removing the solvent in vacuo, afforded product 41 in $99 \%$ of yield with $86 \%$ ee. $[\alpha]_{\mathrm{D}}{ }^{20}=-78.3\left(c 1.0, \mathrm{CHCl}_{3}\right) ;{ }^{1} \mathrm{H}$ NMR $(600 \mathrm{MHz}$, $\left.\mathrm{CDCl}_{3}\right) \delta=7.68(\mathrm{~d}, J=7.4,2 \mathrm{H}), 7.40(\mathrm{t}, J=7.5,2 \mathrm{H}), 7.35(\mathrm{t}, J=7.2,1 \mathrm{H}), 7.23(\mathrm{~d}, J=$ $8.8,1 \mathrm{H}), 6.85(\mathrm{~d}, J=8.9,2 \mathrm{H}), 6.81-6.73(\mathrm{~m}, 3 \mathrm{H}), 6.35(\mathrm{~d}, J=2.9,1 \mathrm{H}), 3.77(\mathrm{~s}, 3 \mathrm{H})$, $3.62(\mathrm{~s}, 3 \mathrm{H}), 1.27(\mathrm{~s}, 9 \mathrm{H}) .{ }^{13} \mathrm{C} \mathrm{NMR}\left(150 \mathrm{MHz}, \mathrm{CDCl}_{3}\right) \delta=174.2,153.7,153.6,140.3$, $137.9,136.9,134.3,128.2,128.1,126.9,120.9,118.3,116.4,114.6,113.6,83.9,80.7$, 55.7, 55.4, 27.9. HR-ESI-MS calcd. for $\mathrm{C}_{26} \mathrm{H}_{29} \mathrm{NO}_{5} \mathrm{Na}[\mathrm{M}+\mathrm{Na}]^{+}$: 458.1938, found: 458.1936. HPLC (Daicel Chiralpak AD-H $(25 \mathrm{~cm})$, Hexanes/IPA = 90/10, flow rate $=$ $0.5 \mathrm{~mL} / \mathrm{min}, \mathrm{I}=280 \mathrm{~nm}$ ) $\mathrm{t}_{\mathrm{R}}=8.71 \mathrm{~min}$ (major), $9.72 \mathrm{~min}$ (minor).

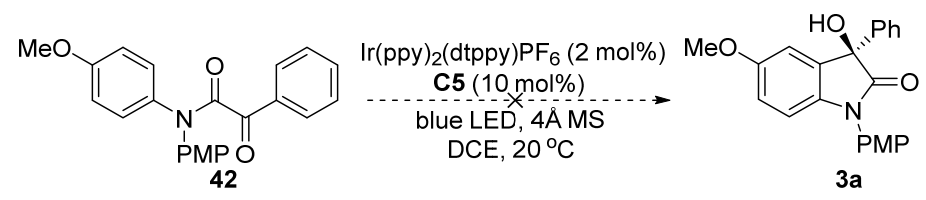

Following the standard procedure, to a $10 \mathrm{~mL}$ flame-dry Schlenk reaction tube equipped with a magnetic stir bar. $42(0.10 \mathrm{mmol}), \operatorname{Ir}(\mathrm{ppy})_{2}(\mathrm{dtbbpy}) \mathrm{PF}_{6}(0.0020 \mathrm{mmol})$, C5 $(0.010 \mathrm{mmol}), 4 \AA \mathrm{MS}(25.0 \mathrm{mg})$, and dry DCE $(1.0 \mathrm{~mL})$ were sequentially added. The reaction mixture was degassed three times by freeze-pump-thaw method. The tube was placed approximately $10 \mathrm{~cm}$ away from a $18 \mathrm{~W}$ blue LED lamp, and the reaction mixture was stirred at $20{ }^{\circ} \mathrm{C}$ under an argon atmosphere. No coupling products were identified after $24 \mathrm{~h}$. 


\section{Crystal Data and Structure Refinement for Compound 8}

Single crystal of $\mathbf{8}$ was obtained from a mixed solution of ethyl acetate/petroleum ether $(1 / 10)$ at room temperature with slow volatilization. The absolute structure of product 8 was determined by X-ray diffraction analysis of a single crystal (Bruker D8 Quest diffractometer). The X-ray data have been deposited at the Cambridge Crystallographic Data Center (CCDC2072968). The stereochemistry of other products was assumed by analogy.

Crystal data for 8: $\mathrm{C}_{22} \mathrm{H}_{18} \mathrm{BrNO}_{4}, M=440.28, a=8.3791(12) \AA, b=23.073(3) \AA, c$ $=10.1816(14) \AA, \alpha=90^{\circ}, \beta=100.393(5)^{\circ}, \gamma=90^{\circ}, V=1936.1(5) \AA^{3}, T=100 .(2) \mathrm{K}$, space group $P 1211, Z=4, \mu(\mathrm{Cu} \mathrm{K \alpha})=3.136 \mathrm{~mm}^{-1}, 30156$ reflections measured, 7515 independent reflections $\left(R_{\text {int }}=0.0777\right)$. The final $R_{l}$ values were $0.0607(I>2 \sigma(I))$. The final $w R\left(F^{2}\right)$ values were $0.1615(I>2 \sigma(I))$. The final $R_{l}$ values were 0.0639 (all data). The final $w R\left(F^{2}\right)$ values were 0.1674 (all data). The goodness of fit on $F^{2}$ was 1.106 . Flack parameter $=0.129(11)$.

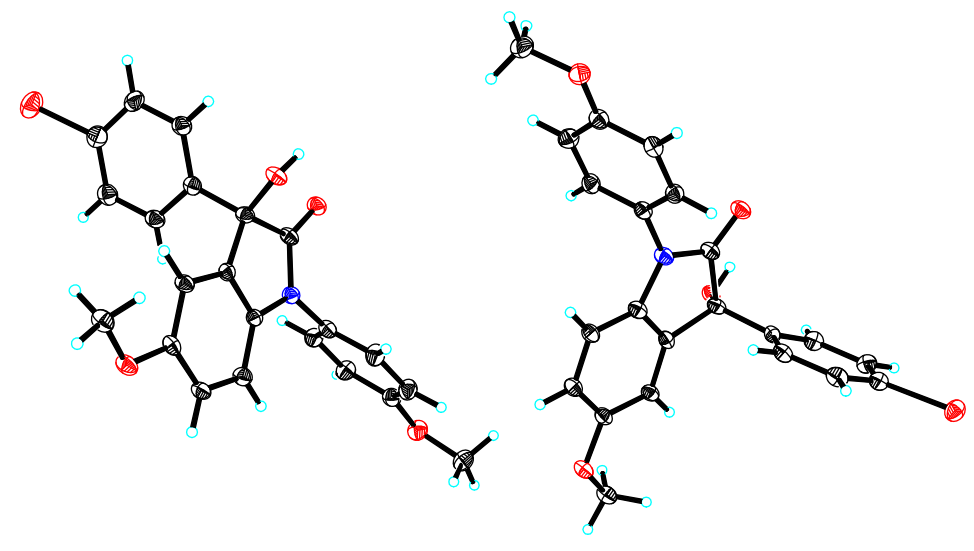

View of the molecules in an asymmetric unit. Displacement ellipsoids are drawn at the $30 \%$ probability level. 


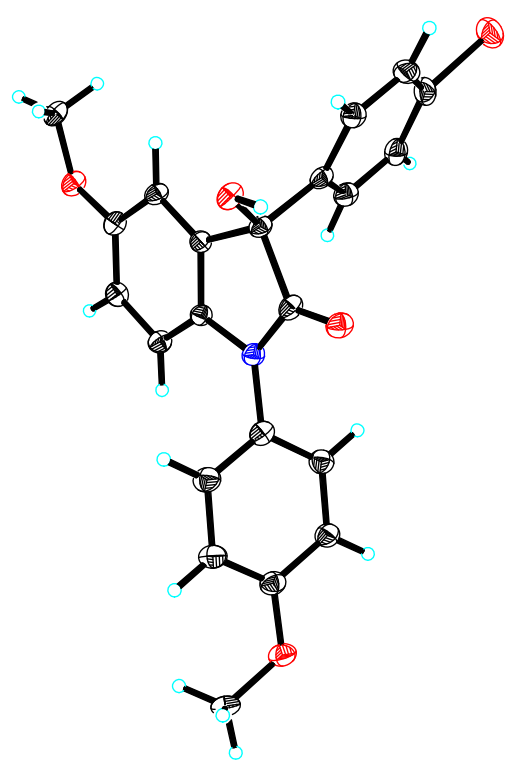

View of a molecule of $\mathbf{8}$ with the atom-labelling scheme.

Displacement ellipsoids are drawn at the $30 \%$ probability level.

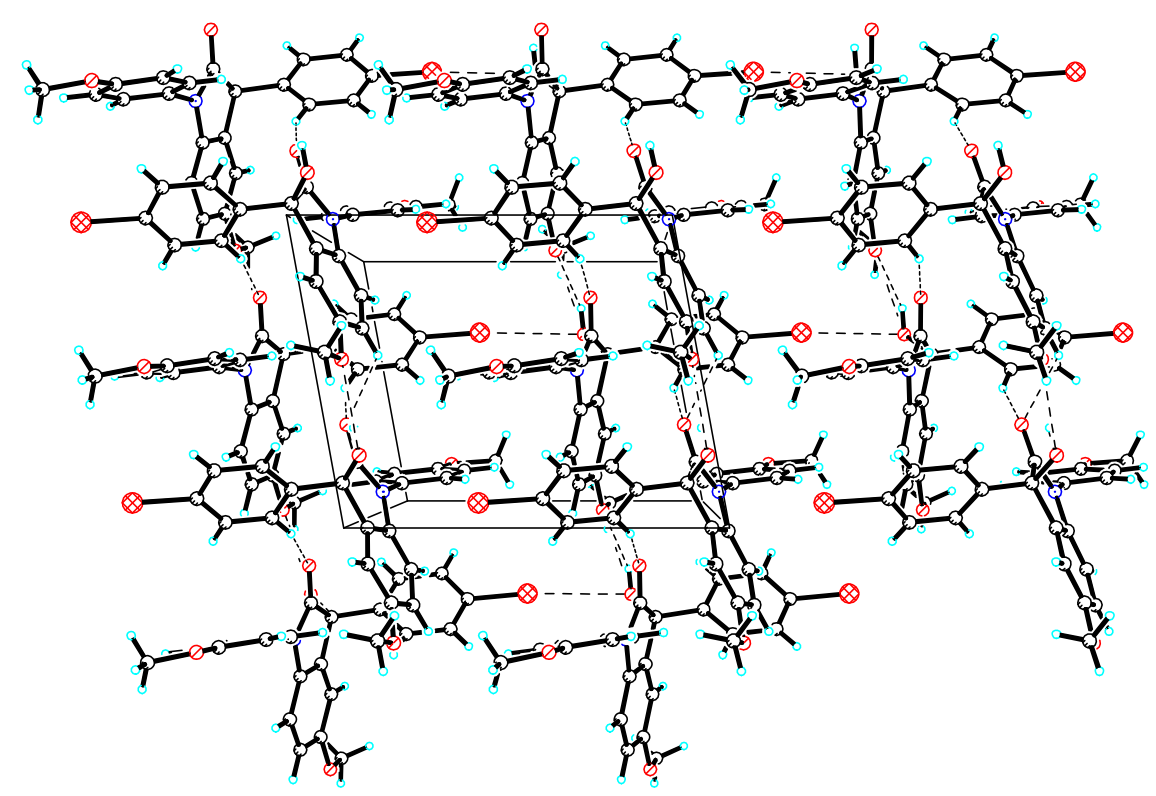

View of the pack drawing of $\mathbf{8}$.

Hydrogen-bonds are shown as dashed lines.

Table S3. Crystal data and structure refinement for 8 .

Identification code

Empirical formula

Formula weight global

$\mathrm{C} 22 \mathrm{H} 18 \mathrm{Br}$ N O4

440.28 
Temperature

Wavelength

Crystal system

Space group

Unit cell dimensions

Volume

Z

Density (calculated)

Absorption coefficient

$\mathrm{F}(000)$

Crystal size

Theta range for data collection

Index ranges

Reflections collected

Independent reflections

Completeness to theta $=73.06^{\circ}$

Absorption correction

Max. and min. transmission

Refinement method

Data / restraints / parameters

Goodness-of-fit on $\mathrm{F}^{2}$

Final $\mathrm{R}$ indices [I $>2 \operatorname{sigma}(\mathrm{I})]$

$\mathrm{R}$ indices (all data)

Absolute structure parameter

Largest diff. peak and hole
100(2) K

$1.54178 \AA$

Monoclinic

P 1211

$\begin{array}{ll}\mathrm{a}=8.3791(12) \AA & \mathrm{a}=90^{\circ} . \\ \mathrm{b}=23.073(3) \AA & \mathrm{b}=100.393(5)^{\circ} . \\ \mathrm{c}=10.1816(14) \AA & \mathrm{g}=90^{\circ} .\end{array}$

1936.1(5) $\AA^{3}$

4

$1.510 \mathrm{Mg} / \mathrm{m}^{3}$

$3.136 \mathrm{~mm}^{-1}$

896

$0.440 \times 0.200 \times 0.120 \mathrm{~mm}^{3}$

3.83 to $73.06^{\circ}$.

$-8<=\mathrm{h}<=10,-28<=\mathrm{k}<=28,-12<=\mathrm{l}<=12$

30156

$7515[\mathrm{R}(\mathrm{int})=0.0777]$

$98.9 \%$

Semi-empirical from equivalents

0.70 and 0.35

Full-matrix least-squares on $\mathrm{F}^{2}$

$7515 / 1 / 511$

1.106

$\mathrm{R} 1=0.0607, \mathrm{wR} 2=0.1615$

$\mathrm{R} 1=0.0639, \mathrm{wR} 2=0.1674$

$0.129(11)$

1.195 and -0.637 e. $\AA^{-3}$ 


\section{Characterization of Products}

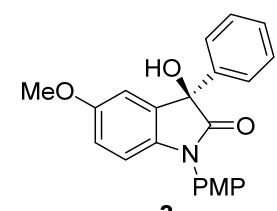

\section{(R)-3-Hydroxy-5-methoxy-1-(4-methoxyphenyl)-3-phenylindolin-2-one}

(3):

Prepared according to general procedures. Colorless oil (35.7 mg, 99\% yield, 96\% ee). Flash column chromatography conditions: petroleum ether/ethyl acetate $=2.5 / 1 .[\alpha]_{\mathrm{D}^{20}}$ $=+26.1\left(c\right.$ 1.0, $\left.\mathrm{CHCl}_{3}\right) ;{ }^{1} \mathrm{H}$ NMR $\left(600 \mathrm{MHz}, \mathrm{CDCl}_{3}\right) \delta=7.48-7.43(\mathrm{~m}, 2 \mathrm{H}), 7.37-$ $7.28(\mathrm{~m}, 5 \mathrm{H}), 7.03-6.98(\mathrm{~m}, 2 \mathrm{H}), 6.91(\mathrm{~d}, J=2.5,1 \mathrm{H}), 6.78(\mathrm{dd}, J=8.6,2.5,1 \mathrm{H})$, $6.74(\mathrm{~d}, J=8.6,1 \mathrm{H}), 3.85(\mathrm{~s}, 3 \mathrm{H}), 3.74(\mathrm{~s}, 3 \mathrm{H}) .{ }^{13} \mathrm{C} \mathrm{NMR}\left(150 \mathrm{MHz}, \mathrm{CDCl}_{3}\right) \delta=177.0$, $159.2,156.8,140.5,137.2,132.5,128.7,128.3,127.7,126.9,125.3,115.0,114.8,111.6$, 110.6, 78.4, 55.8, 55.6. HR-ESI-MS calcd. for $\mathrm{C}_{22} \mathrm{H}_{19} \mathrm{NO}_{4} \mathrm{Na}[\mathrm{M}+\mathrm{Na}]^{+}$: 384.1206, found: 384.1202. HPLC (Daicel Chiralpak AD-H (25 cm), Hexanes/IPA = 50/50, flow rate $=0.5 \mathrm{~mL} / \mathrm{min}, \mathrm{I}=280 \mathrm{~nm}$ ) $\mathrm{t}_{\mathrm{R}}=39.52 \mathrm{~min}$ (major), $60.71 \mathrm{~min}$ (minor).

\section{Scale-up Experiment}

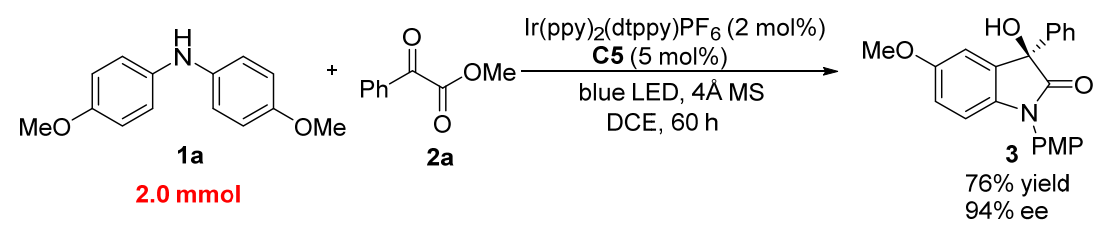

To an oven dried $100 \mathrm{~mL}$ round-bottom flask equipped with a magnetic stir bar. Diarylamine 1a (458.2 mg, $2.0 \mathrm{mmol})$, $\alpha$-ketoester 2a (394.0 mg, $2.4 \mathrm{mmol})$, $\operatorname{Ir}($ ppy)2(dtbbpy)PF6 (37.0 mg, $0.040 \mathrm{mmol}$ ) ), C5 (67.0 mg, $0.10 \mathrm{mmol}), 4 \AA \mathrm{MS}$ (500 $\mathrm{mg})$, and dry DCE (20 mL) were sequentially added. The reaction mixture was degassed three times by freeze-pump-thaw method. The flask was placed approximately $10 \mathrm{~cm}$ away from two $18 \mathrm{~W}$ blue LED lamp. The reaction mixture was stirred at room temperature under an argon atmosphere for $60 \mathrm{~h}$. The reaction mixture was concentrated. The residue was purified by silica gel chromatography (petroleum ether/ethyl acetate $=$ 2.5/1) to afford products 3 (548.0 $\mathrm{mg}, 76 \%$ yield, $94 \%$ ee). 


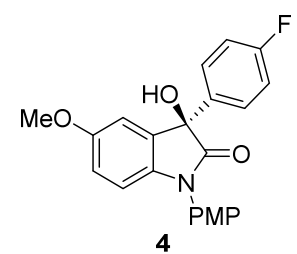

(R)-3-(4-Fluorophenyl)-3-hydroxy-5-methoxy-1-(4-methoxyphenyl)indolin-2-one

(4): Prepared according to general procedures. Colorless oil (34.1 mg, 90\% yield, 93\% ee). Flash column chromatography conditions: petroleum ether/ethyl acetate $=2.5 / 1$. $[\alpha]_{\mathrm{D}}^{20}=+56.9\left(c\right.$ 1.0, $\left.\mathrm{CHCl}_{3}\right) ;{ }^{1} \mathrm{H} \mathrm{NMR}\left(600 \mathrm{MHz}, \mathrm{CDCl}_{3}\right) \delta=7.42(\mathrm{dd}, J=8.7,5.3$, 2H), $7.29(\mathrm{~d}, J=8.9,2 \mathrm{H}), 6.89(\mathrm{~d}, J=2.5,1 \mathrm{H}), 6.78(\mathrm{dd}, J=8.6,2.5,1 \mathrm{H}), 6.73(\mathrm{~d}, J=$ 8.6, 1H), $4.51(\mathrm{~s}, 1 \mathrm{H}), 3.84(\mathrm{~s}, 3 \mathrm{H}), 3.72(\mathrm{~s}, 3 \mathrm{H}) .{ }^{13} \mathrm{C} \mathrm{NMR}\left(150 \mathrm{MHz}, \mathrm{CDCl}_{3}\right) \delta=177.0$, $163.5,161.9,159.2,156.9,137.1,136.3,132.6,127.6,127.5,127.4,126.8,115.6,115.4$, 115.0, 114.8, 111.6, 110.7, 78.0, 55.8, 55.6. $\left.{ }^{19} \mathrm{~F} \mathrm{NMR} \mathrm{(565} \mathrm{MHz,} \mathrm{CDCl}_{3}\right) \delta=-113.8$. HR-ESI-MS calcd. for $\mathrm{C}_{22} \mathrm{H}_{18} \mathrm{NO}_{4} \mathrm{FNa}[\mathrm{M}+\mathrm{Na}]^{+}:$402.1112, found: 402.1109. HPLC (Daicel Chiralpak AD-H $(25 \mathrm{~cm})$, Hexanes $/ \mathrm{IPA}=50 / 50$, flow rate $=0.5 \mathrm{~mL} / \mathrm{min}, \mathrm{I}=$ $280 \mathrm{~nm}) \mathrm{t}_{\mathrm{R}}=34.53 \mathrm{~min}$ (major), $56.24 \mathrm{~min}$ (minor).

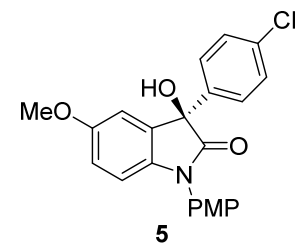

(R)-3-(4-Chlorophenyl)-3-hydroxy-5-methoxy-1-(4-methoxyphenyl)indolin-2-one

(5) : Prepared according to general procedures. Colorless oil (33.2 mg, 84\% yield, 92\% ee). Flash column chromatography conditions: petroleum ether/ethyl acetate $=2.5 / 1$. $[\alpha]_{\mathrm{D}}{ }^{20}=+46.3\left(c 1.0, \mathrm{CHCl}_{3}\right) ;{ }^{1} \mathrm{H} \mathrm{NMR}\left(600 \mathrm{MHz}, \mathrm{CDCl}_{3}\right) \delta=7.37(\mathrm{~d}, J=8.5,2 \mathrm{H})$, $7.30(\mathrm{~d}, J=7.1,4 \mathrm{H}), 7.01(\mathrm{~d}, J=8.8,2 \mathrm{H}), 6.88(\mathrm{~d}, J=2.3,1 \mathrm{H}), 6.79(\mathrm{dd}, J=8.6,2.4$, $1 \mathrm{H}), 6.74(\mathrm{~d}, J=8.6,1 \mathrm{H}), 4.23(\mathrm{~s}, 1 \mathrm{H}), 3.85(\mathrm{~s}, 3 \mathrm{H}), 3.73(\mathrm{~s}, 3 \mathrm{H}) .{ }^{13} \mathrm{C} \mathrm{NMR}(150 \mathrm{MHz}$, $\left.\mathrm{CDCl}_{3}\right) \delta=176.7,159.3,156.9,139.0,137.1,134.3,132.2,128.8,127.6,126.9,126.7$, 115.0, 111.5, 110.7, 78.0, 55.8, 55.6. HR-ESI-MS calcd. for $\mathrm{C}_{22} \mathrm{H}_{19} \mathrm{NO}_{4} \mathrm{Cl}[\mathrm{M}+\mathrm{H}]^{+}$: 396.0997, found: 396.0998. HPLC (Daicel Chiralpak AD-H (25 cm), Hexanes/IPA = $50 / 50$, flow rate $=0.5 \mathrm{~mL} / \mathrm{min}, \mathrm{I}=280 \mathrm{~nm}$ ) $\mathrm{t}_{\mathrm{R}}=46.30 \mathrm{~min}$ (major), $82.18 \mathrm{~min}$ (minor). 


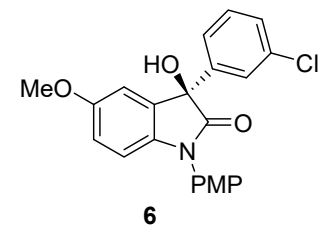

(R)-3-(3-Chlorophenyl)-3-hydroxy-5-methoxy-1-(4-methoxyphenyl)indolin-2-one

(6): Prepared according to general procedures. White powder $(34.4 \mathrm{mg}, 87 \%$ yield, $92 \%$

ee). mp: $143.8-145.3{ }^{\circ} \mathrm{C}$. Flash column chromatography conditions: petroleum ether/ethyl acetate $=2.5 / 1 .[\alpha]_{\mathrm{D}}{ }^{20}=+100.8\left(c\right.$ 1.0, $\left.\mathrm{CHCl}_{3}\right) ;{ }^{1} \mathrm{H} \mathrm{NMR}\left(600 \mathrm{MHz}, \mathrm{CDCl}_{3}\right)$ $\delta=7.44(\mathrm{~d}, J=1.5,1 \mathrm{H}), 7.34-7.18(\mathrm{~m}, 5 \mathrm{H}), 7.03-6.95(\mathrm{~m}, 2 \mathrm{H}), 6.85(\mathrm{~d}, J=2.3$, 1H), $6.77(\mathrm{dd}, J=8.6,2.3,1 \mathrm{H}), 6.74-6.69(\mathrm{~m}, 1 \mathrm{H}), 4.56(\mathrm{~s}, 1 \mathrm{H}), 3.82(\mathrm{~s}, 3 \mathrm{H}), 3.70$ (s, 3H). ${ }^{13} \mathrm{C} \mathrm{NMR}\left(150 \mathrm{MHz}, \mathrm{CDCl}_{3}\right) \delta 176.6,159.3,156.9,142.6,137.0,134.6,132.3$, 130.0, 128.5, 127.6, 126.7, 125.7, 123.7, 115.0, 111.5, 110.8, 78.1, 55.9, 55.6. HR-ESIMS calcd. for $\mathrm{C}_{22} \mathrm{H}_{19} \mathrm{NO}_{4} \mathrm{Cl}[\mathrm{M}+\mathrm{H}]^{+}$: 396.0997, found: 396.0998. HPLC (Daicel Chiralpak AD-H $(25 \mathrm{~cm})$, Hexanes $/ \mathrm{IPA}=50 / 50$, flow rate $=0.5 \mathrm{~mL} / \mathrm{min}, \mathrm{I}=280 \mathrm{~nm})$ $\mathrm{t}_{\mathrm{R}}=30.66 \mathrm{~min}$ (major), $42.01 \mathrm{~min}$ (minor).

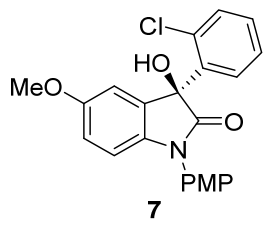

(S)-3-(2-Chlorophenyl)-3-hydroxy-5-methoxy-1-(4-methoxyphenyl)indolin-2-one

(7): Prepared according to general procedures. White powder $(39.1 \mathrm{mg}, 99 \%$ yield, $81 \%$ ee). mp: $151.7-153.5{ }^{\circ} \mathrm{C}$. Flash column chromatography conditions: petroleum ether/ethyl acetate $=2.5 / 1 .[\alpha]_{\mathrm{D}}{ }^{20}=+65.4\left(c\right.$ 1.0, $\left.\mathrm{CHCl}_{3}\right) ;{ }^{1} \mathrm{H}$ NMR $(600 \mathrm{MHz}, \mathrm{MeOD})$ $\delta 8.22(\mathrm{dd}, J=7.9,1.3 \mathrm{~Hz}, 1 \mathrm{H}), 7.49-7.43(\mathrm{~m}, 1 \mathrm{H}), 7.40-7.28(\mathrm{~m}, 4 \mathrm{H}), 7.13-7.04$ $(\mathrm{m}, 2 \mathrm{H}), 6.82(\mathrm{dd}, J=8.6,2.6 \mathrm{~Hz}, 1 \mathrm{H}), 6.68(\mathrm{~d}, J=8.6 \mathrm{~Hz}, 1 \mathrm{H}), 6.56(\mathrm{~d}, J=2.5 \mathrm{~Hz}$, 1H), 3.85 (s, 3H), $3.66(\mathrm{~s}, 3 \mathrm{H}) .{ }^{13} \mathrm{C}$ NMR (150 MHz, MeOD) $\delta$ 176.2, 159.5, 156.8, 138.6, 138.0, 131.8, 131.1, 129.8, 129.4, 128.6, 127.4, 127.1, 126.6, 114.6, 113.9, 110.9, 109.9, 76.9, 54.8, 54.7. HR-ESI-MS calcd. for $\mathrm{C}_{22} \mathrm{H}_{19} \mathrm{NO}_{4} \mathrm{Cl}[\mathrm{M}+\mathrm{H}]^{+}: 396.0997$, found: 396.1001. HPLC (Daicel Chiralpak AD-H $(25 \mathrm{~cm})$, Hexanes/IPA $=50 / 50$, flow rate $=$ 
$0.5 \mathrm{~mL} / \mathrm{min}, \mathrm{I}=280 \mathrm{~nm}$ ) $\mathrm{t}_{\mathrm{R}}=17.47 \mathrm{~min}$ (major), $41.34 \mathrm{~min}$ (minor).

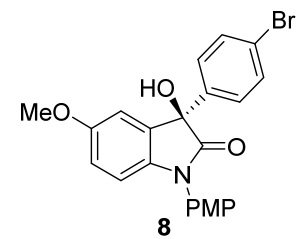

(R)-3-(4-Bromophenyl)-3-hydroxy-5-methoxy-1-(4-methoxyphenyl)indolin-2-one (8): Prepared according to general procedures. White solid (38.2 mg, 87\% yield, 92\% ee), mp: $154.6-156.5{ }^{\circ} \mathrm{C}$. Flash column chromatography conditions: petroleum ether/acetone $=3 / 1 .[\alpha]_{\mathrm{D}}^{20}=+41.2\left(c 1.0, \mathrm{CHCl}_{3}\right) ;{ }^{1} \mathrm{H}$ NMR $\left(600 \mathrm{MHz}, \mathrm{CDCl}_{3}\right) \delta=$ $7.44(\mathrm{~d}, J=8.3,2 \mathrm{H}), 7.29(\mathrm{t}, J=8.7,4 \mathrm{H}), 7.00(\mathrm{~d}, J=8.7,2 \mathrm{H}), 6.87(\mathrm{~d}, J=2.2,1 \mathrm{H})$, $6.78(\mathrm{dd}, J=8.6,2.3,1 \mathrm{H}), 6.73(\mathrm{~d}, J=8.6,1 \mathrm{H}), 4.57(\mathrm{~s}, 1 \mathrm{H}), 3.83(\mathrm{~s}, 3 \mathrm{H}), 3.70(\mathrm{~s}, 3 \mathrm{H})$. ${ }^{13} \mathrm{C} \mathrm{NMR}\left(150 \mathrm{MHz}, \mathrm{CDCl}_{3}\right) \delta=176.7,159.3,156.9,139.6,137.0,132.3,131.7,127.6$, 127.3, 126.7, 122.4, 115.0, 111.4, 110.7, 78.1, 55.8, 55.6. HR-ESI-MS calcd. for $\mathrm{C}_{22} \mathrm{H}_{19} \mathrm{NO}_{4} \mathrm{Br}\left[\mathrm{M}+\mathrm{H}^{+}:\right.$440.0492, found: 440.0493. HPLC (Daicel Chiralpak AD-H $(25 \mathrm{~cm})$, Hexanes $/ \mathrm{IPA}=50 / 50$, flow rate $=0.5 \mathrm{~mL} / \mathrm{min}, \mathrm{I}=280 \mathrm{~nm}) \mathrm{t}_{\mathrm{R}}=55.17 \mathrm{~min}$ (major), $99.41 \mathrm{~min}$ (minor).

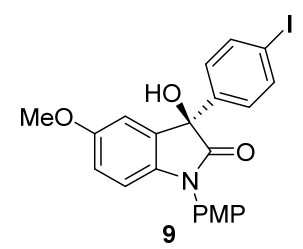

(R)-3-Hydroxy-3-(4-iodophenyl)-5-methoxy-1-(4-methoxyphenyl)indolin-2-one

(9): Prepared according to general procedures. White foam (39.9 mg, 82\% yield, 91\% ee). mp: $165.6-167.1{ }^{\circ} \mathrm{C}$. Flash column chromatography conditions: petroleum ether/ethyl acetate $=2.5 / 1 .[\alpha]_{\mathrm{D}}{ }^{20}=+58.7\left(c 1.0, \mathrm{CHCl}_{3}\right) ;{ }^{1} \mathrm{H} \mathrm{NMR}\left(600 \mathrm{MHz}, \mathrm{CDCl}_{3}\right)$ $\delta=7.62(\mathrm{~d}, J=8.5,2 \mathrm{H}), 7.26(\mathrm{~d}, J=8.9,2 \mathrm{H}), 7.15(\mathrm{~d}, J=8.5,2 \mathrm{H}), 6.98(\mathrm{~d}, J=8.9$, $2 \mathrm{H}), 6.84(\mathrm{~d}, J=2.5,1 \mathrm{H}), 6.76(\mathrm{dd}, J=8.6,2.5,1 \mathrm{H}), 6.71(\mathrm{~d}, J=8.6,1 \mathrm{H}), 4.61(\mathrm{~s}, 1 \mathrm{H})$, $3.82(\mathrm{~s}, 3 \mathrm{H}), 3.70(\mathrm{~s}, 3 \mathrm{H}) .{ }^{13} \mathrm{C} \mathrm{NMR}\left(150 \mathrm{MHz}, \mathrm{CDCl}_{3}\right) \delta=176.7,159.2,156.9,140.3$, $137.7,137.0,132.3,127.6,127.5,126.7,115.0,111.4,110.7,94.2,78.2$, 55.9, 55.6. HR-ESI-MS calcd. for $\mathrm{C}_{22} \mathrm{H}_{18} \mathrm{NO}_{4} \mathrm{INa}[\mathrm{M}+\mathrm{Na}]^{+}:$510.0173, found: 510.0174. HPLC 
(Daicel Chiralpak AD-H $(25 \mathrm{~cm})$, Hexanes $/ \mathrm{IPA}=50 / 50$, flow rate $=0.5 \mathrm{~mL} / \mathrm{min}, \mathrm{I}=$ $280 \mathrm{~nm}) \mathrm{t}_{\mathrm{R}}=74.86 \mathrm{~min}$ (major), $118.97 \mathrm{~min}$ (minor).

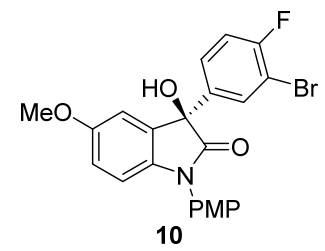

(R)-3-(3-Bromo-4-fluorophenyl)-3-hydroxy-5-methoxy-1-(4-methoxyphenyl)

indolin-2-one (10): Prepared according to general procedures. Colorless oil (33.8 mg, 74\% yield, 90\% ee). Flash column chromatography conditions: petroleum ether/ethyl acetate $=2.5 / 1 .[\alpha]_{\mathrm{D}}{ }^{20}=+40.7\left(c 1.0, \mathrm{CHCl}_{3}\right) ;{ }^{1} \mathrm{H} \mathrm{NMR}\left(600 \mathrm{MHz}, \mathrm{CDCl}_{3}\right) \delta=7.68$ $(\mathrm{dd}, J=6.4,2.0,1 \mathrm{H}), 7.36-7.31(\mathrm{~m}, 1 \mathrm{H}), 7.30(\mathrm{~d}, J=8.8,2 \mathrm{H}), 7.06(\mathrm{t}, J=8.4,1 \mathrm{H})$, $7.02(\mathrm{~d}, J=8.8,2 \mathrm{H}), 6.88(\mathrm{~d}, J=2.4,1 \mathrm{H}), 6.81(\mathrm{dd}, J=8.6,2.4,1 \mathrm{H}), 6.76(\mathrm{~d}, J=8.6$, $1 \mathrm{H}), 4.84(\mathrm{~s}, 1 \mathrm{H}), 3.86(\mathrm{~s}, 3 \mathrm{H}), 3.75(\mathrm{~s}, 3 \mathrm{H}) .{ }^{13} \mathrm{C} \mathrm{NMR}\left(150 \mathrm{MHz}, \mathrm{CDCl}_{3}\right) \delta 176.6,159.7$, $159.3,158.1,157.0,137.9,137.9,136.9,132.2,130.9,127.6,126.5,126.5,126.4,116.6$, 116.5, 115.0, 115.0, 111.5, 110.9, 109.4, 109.2, 77.6, 55.9, 55.6. ${ }^{19} \mathrm{~F}$ NMR (565 MHz, $\left.\mathrm{CDCl}_{3}\right) \delta=-107.8$. HR-ESI-MS calcd. for $\mathrm{C}_{22} \mathrm{H}_{18} \mathrm{NO}_{4} \mathrm{FBr}[\mathrm{M}+\mathrm{H}]^{+}:$458.0398, found: 458.0399. HPLC (Daicel Chiralpak OZ-H $(25 \mathrm{~cm})$, Hexanes/IPA $=50 / 50$, flow rate $=$ $0.5 \mathrm{~mL} / \mathrm{min}, \mathrm{I}=280 \mathrm{~nm}$ ) $\mathrm{t}_{\mathrm{R}}=10.37 \mathrm{~min}$ (major), $13.63 \mathrm{~min}$ (minor).

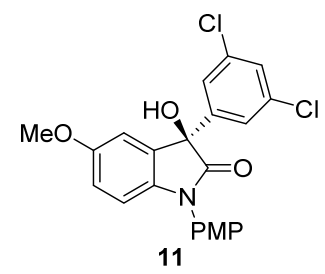

(R)-3-(3,5-Dichlorophenyl)-3-hydroxy-5-methoxy-1-(4-methoxyphenyl)indolin-2one (11): Prepared according to general procedures. Colorless oil (30.1 mg, 70\% yield, $82 \%$ ee). Flash column chromatography conditions: petroleum ether/ethyl acetate $=$ 2.5/1. $[\alpha]_{\mathrm{D}}{ }^{20}=+36.3\left(c 1.0, \mathrm{CHCl}_{3}\right) ;{ }^{1} \mathrm{H} \mathrm{NMR}\left(600 \mathrm{MHz}, \mathrm{CDCl}_{3}\right) \delta=7.36-7.27(\mathrm{~m}$, $5 \mathrm{H}), 7.04-6.99(\mathrm{~m}, 2 \mathrm{H}), 6.83(\mathrm{~d}, J=2.5,1 \mathrm{H}), 6.80(\mathrm{dd}, J=8.6,2.5,1 \mathrm{H}), 6.75(\mathrm{~d}, J=$ 8.6, 1H), $4.62(\mathrm{~s}, 1 \mathrm{H}), 3.85(\mathrm{~s}, 3 \mathrm{H}), 3.73(\mathrm{~s}, 3 \mathrm{H}) .{ }^{13} \mathrm{C} \mathrm{NMR}\left(150 \mathrm{MHz}, \mathrm{CDCl}_{3}\right) \delta 176.2$, $159.4,157.0,143.9,136.9,135.3,131.8,128.5,127.6,126.4,124.1,115.2,115.0,111.5$, 
111.0, 77.8, 55.9, 55.6. HR-ESI-MS calcd. for $\mathrm{C}_{22} \mathrm{H}_{17} \mathrm{NO}_{4} \mathrm{Cl}_{2} \mathrm{Na}[\mathrm{M}+\mathrm{Na}]^{+}$: 452.0427, found: 452.0423.. HPLC (Daicel Chiralpak OZ-H (25 cm), Hexanes/IPA = 80/20, flow rate $=0.5 \mathrm{~mL} / \mathrm{min}, \mathrm{I}=280 \mathrm{~nm}) \mathrm{t}_{\mathrm{R}}=15.62 \mathrm{~min}$ (major), $19.66 \mathrm{~min}$ (minor).

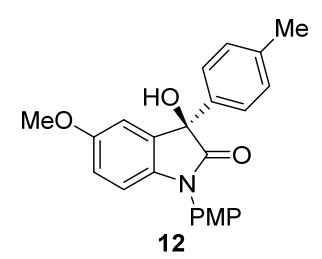

(R)-3-Hydroxy-5-methoxy-1-(4-methoxyphenyl)-3-(p-tolyl)indolin-2-one

Prepared according to general procedures. Colorless oil (27.2 mg, 72\% yield, 94\% ee). Flash column chromatography conditions: petroleum ether/ethyl acetate $=2.5 / 1 .[\alpha]_{\mathrm{D}}{ }^{20}$ $=+83.9\left(c\right.$ 1.0, $\left.\mathrm{CHCl}_{3}\right) ;{ }^{1} \mathrm{H} \mathrm{NMR}\left(600 \mathrm{MHz}, \mathrm{CDCl}_{3}\right) \delta=7.36(\mathrm{~d}, J=8.2,2 \mathrm{H}), 7.33(\mathrm{~d}$, $J=8.9,2 \mathrm{H}), 7.15(\mathrm{~d}, J=8.0,2 \mathrm{H}), 7.01(\mathrm{~d}, J=8.9,2 \mathrm{H}), 6.94(\mathrm{~d}, J=2.5,1 \mathrm{H}), 6.78(\mathrm{dd}$, $J=8.6,2.5,1 \mathrm{H}), 6.74(\mathrm{~d}, J=8.6,1 \mathrm{H}), 4.06(\mathrm{~s}, 1 \mathrm{H}), 3.84(\mathrm{~s}, 3 \mathrm{H}), 3.73(\mathrm{~s}, 3 \mathrm{H}), 2.34(\mathrm{~s}$, $3 \mathrm{H}) .{ }^{13} \mathrm{C} \mathrm{NMR}\left(150 \mathrm{MHz}, \mathrm{CDCl}_{3}\right) \delta 177.2,159.2,156.8,138.1,137.6,137.2,132.7$, $129.4,127.7,127.0,125.3,114.9,114.8,111.5,110.5,78.3,55.8,55.6,21.1$. HR-ESIMS calcd. for $\mathrm{C}_{23} \mathrm{H}_{22} \mathrm{NO}_{4}[\mathrm{M}+\mathrm{H}]^{+}$: 376.1543, found: 376.1543. HPLC (Daicel Chiralpak AD-H $(25 \mathrm{~cm})$, Hexanes $/ \mathrm{IPA}=50 / 50$, flow rate $=0.5 \mathrm{~mL} / \mathrm{min}, \mathrm{I}=280 \mathrm{~nm}$ ) $\mathrm{t}_{\mathrm{R}}=71.49 \min$ (major), $97.34 \mathrm{~min}$ (minor).

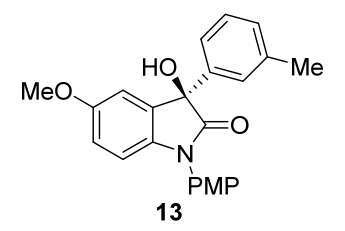

(R)-3-Hydroxy-5-methoxy-1-(4-methoxyphenyl)-3-(m-tolyl)indolin-2-one

Prepared according to general procedures. Colorless oil (31.9 mg, 85\% yield, 95\% ee). Flash column chromatography conditions: petroleum ether/ethyl acetate $=2.5 / 1 .[\alpha]_{\mathrm{D}}{ }^{20}$ $=+47.4\left(c 1.0, \mathrm{CHCl}_{3}\right) ;{ }^{1} \mathrm{H} \mathrm{NMR}\left(600 \mathrm{MHz}, \mathrm{CDCl}_{3}\right) \delta=7.34(\mathrm{~d}, J=9.0,3 \mathrm{H}), 7.25-$ $7.20(\mathrm{~m}, 2 \mathrm{H}), 7.13(\mathrm{~d}, J=6.3,1 \mathrm{H}), 7.01(\mathrm{~d}, J=8.9,2 \mathrm{H}), 6.93(\mathrm{~d}, J=2.4,1 \mathrm{H}), 6.79(\mathrm{dd}$, $J=8.6,2.5,1 \mathrm{H}), 6.75(\mathrm{~d}, J=8.6,1 \mathrm{H}), 4.22(\mathrm{~s}, 1 \mathrm{H}), 3.83(\mathrm{~s}, 3 \mathrm{H}), 3.72(\mathrm{~s}, 3 \mathrm{H}), 2.34(\mathrm{~s}$, $3 \mathrm{H}) .{ }^{13} \mathrm{C} \mathrm{NMR}\left(150 \mathrm{MHz}, \mathrm{CDCl}_{3}\right) \delta 177.2,159.2,156.8,140.5,138.3,137.2,132.9$, 
$129.1,128.6,127.7,127.0,125.9,122.5,114.9,114.7,111.6,110.5,78.4,55.8,55.6$, 21.6. HR-ESI-MS calcd. for $\mathrm{C}_{23} \mathrm{H}_{21} \mathrm{NO}_{4} \mathrm{Na}[\mathrm{M}+\mathrm{Na}]^{+}$: 398.1363 , found: 398.1366 . HPLC (Daicel Chiralpak OZ-H $(25 \mathrm{~cm})$, Hexanes $/ \mathrm{IPA}=50 / 50$, flow rate $=0.5 \mathrm{~mL} / \mathrm{min}$, $\mathrm{I}=280 \mathrm{~nm}) \mathrm{t}_{\mathrm{R}}=23.04 \mathrm{~min}$ (major), $17.36 \mathrm{~min}$ (minor).

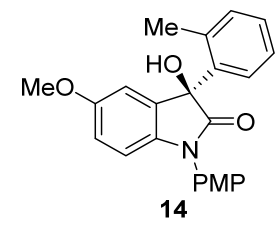

(R)-3-Hydroxy-5-methoxy-1-(4-methoxyphenyl)-3-(o-tolyl)indolin-2-one

(14):

Prepared according to general procedures. Colorless oil (34.9 mg, 93\% yield, 86\% ee).

Flash column chromatography conditions: petroleum ether/ethyl acetate $=2.5 / 1 .[\alpha]_{\mathrm{D}}{ }^{20}$ $=+68.2\left(c\right.$ 1.0, $\left.\mathrm{CHCl}_{3}\right) ;{ }^{1} \mathrm{H} \mathrm{NMR}\left(600 \mathrm{MHz}, \mathrm{CDCl}_{3}\right) \delta=7.99(\mathrm{~d}, J=7.7,1 \mathrm{H}), 7.37(\mathrm{~d}$, $J=8.8,2 \mathrm{H}), 7.30(\mathrm{t}, J=7.4,1 \mathrm{H}), 7.25-7.23(\mathrm{~m}, 1 \mathrm{H}), 7.09(\mathrm{~d}, J=7.3,1 \mathrm{H}), 7.04(\mathrm{~d}, J$ $=8.8,2 \mathrm{H}), 6.79-6.74(\mathrm{~m}, 2 \mathrm{H}), 6.67(\mathrm{~d}, J=2.3,1 \mathrm{H}), 3.85(\mathrm{~s}, 3 \mathrm{H}), 3.67(\mathrm{~s}, 3 \mathrm{H}), 2.01$ (s, 3H). ${ }^{13} \mathrm{C} \mathrm{NMR}\left(150 \mathrm{MHz}, \mathrm{CDCl}_{3}\right) \delta 176.3,159.2,156.8,138.1,137.9,134.5,131.5$, $131.2,128.3,127.4,127.0,126.2,126.1,115.0,114.9,111.6,110.4,77.9,55.8,55.6$, 19.5. HR-ESI-MS calcd. for $\mathrm{C}_{23} \mathrm{H}_{21} \mathrm{NO}_{4} \mathrm{Na}[\mathrm{M}+\mathrm{Na}]^{+}$: 398.1363 , found: 398.1360 . HPLC (Daicel Chiralpak OD-H $(25 \mathrm{~cm})$, Hexanes $/ \mathrm{IPA}=50 / 50$, flow rate $=0.5 \mathrm{~mL} / \mathrm{min}$, $\mathrm{I}=280 \mathrm{~nm}$ ) $\mathrm{t}_{\mathrm{R}}=33.37 \mathrm{~min}$ (major), $16.55 \mathrm{~min}$ (minor).

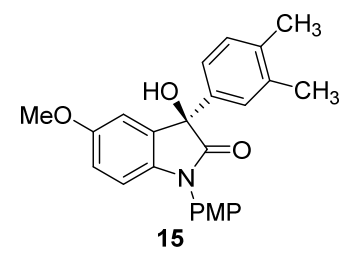

(R)-3-(3,4-Dimethylphenyl)-3-hydroxy-5-methoxy-1-(4-methoxyphenyl)indolin-2one (15): Prepared according to general procedures. White oil $(26.1 \mathrm{mg}, 67 \%$ yield, $95 \%$ ee). Flash column chromatography conditions: petroleum ether/ethyl acetate $=2.5 / 1$. $[\alpha]_{\mathrm{D}}{ }^{20}=+33.9\left(c 1.0, \mathrm{CHCl}_{3}\right) ;{ }^{1} \mathrm{H}$ NMR $\left(600 \mathrm{MHz}, \mathrm{CDCl}_{3}\right) \delta=7.35(\mathrm{~d}, J=8.8,2 \mathrm{H})$, 7.27 (s, 1H), $7.17(\mathrm{dd}, J=7.9,1.7,1 \mathrm{H}), 7.11(\mathrm{~d}, J=7.9,1 \mathrm{H}), 7.02(\mathrm{~d}, J=8.9,2 \mathrm{H}), 6.94$ $(\mathrm{d}, J=2.5,1 \mathrm{H}), 6.78(\mathrm{dd}, J=8.6,2.5,1 \mathrm{H}), 6.75(\mathrm{~d}, J=8.6,1 \mathrm{H}), 3.85(\mathrm{~s}, 3 \mathrm{H}), 3.74(\mathrm{~s}$, 
3H), $2.25(\mathrm{~s}, 6 \mathrm{H}) .{ }^{13} \mathrm{C} \mathrm{NMR}\left(150 \mathrm{MHz}, \mathrm{CDCl}_{3}\right) \delta 177.2,159.2,156.7,137.9,137.2$, $137.0,136.8,132.7,129.9,127.7,127.0,126.4,122.7,114.9,114.7,111.50,110.49$, 78.3, 55.8, 55.6, 20.0, 19.5. HR-ESI-MS calcd. for $\mathrm{C}_{24} \mathrm{H}_{23} \mathrm{NO}_{4} \mathrm{Na}[\mathrm{M}+\mathrm{Na}]^{+}$: 412.1519, found: 412.1523. HPLC (Daicel Chiralpak AD-H $(25 \mathrm{~cm})$, Hexanes/IPA = 50/50, flow rate $=0.5 \mathrm{~mL} / \mathrm{min}, \mathrm{I}=280 \mathrm{~nm}$ ) $\mathrm{t}_{\mathrm{R}}=64.00 \mathrm{~min}$ (major), $51.08 \mathrm{~min}$ (minor).

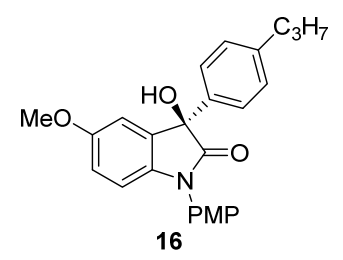

(R)-3-Hydroxy-5-methoxy-1-(4-methoxyphenyl)-3-(4-propylphenyl)indolin-2-one (16) : Prepared according to general procedures. Colorless oil (20.9 mg, 52\% yield, 94\% ee). Flash column chromatography conditions: petroleum ether/ethyl acetate $=2.5 / 1$. $[\alpha]_{\mathrm{D}}^{20}=+61.7\left(c 1.0, \mathrm{CHCl}_{3}\right) ;{ }^{1} \mathrm{H} \mathrm{NMR}\left(600 \mathrm{MHz}, \mathrm{CDCl}_{3}\right) \delta=7.38(\mathrm{~d}, J=8.2,2 \mathrm{H})$, $7.34(\mathrm{dd}, J=8.7,1.7,2 \mathrm{H}), 7.16(\mathrm{~d}, J=7.8,2 \mathrm{H}), 7.05-6.98(\mathrm{~m}, 2 \mathrm{H}), 6.95(\mathrm{~d}, J=2.3$, $1 \mathrm{H}), 6.78(\mathrm{dd}, J=8.6,2.3,1 \mathrm{H}), 6.74(\mathrm{~d}, J=8.6,1 \mathrm{H}), 3.84(\mathrm{~s}, 3 \mathrm{H}), 3.74(\mathrm{~s}, 3 \mathrm{H}), 2.57(\mathrm{t}$, $J=7.5,2 \mathrm{H}), 1.70-1.55(\mathrm{~m}, 2 \mathrm{H}), 0.94(\mathrm{t}, J=7.3,3 \mathrm{H}) .{ }^{13} \mathrm{C} \mathrm{NMR}\left(150 \mathrm{MHz}, \mathrm{CDCl}_{3}\right) \delta$ $=177.1,159.2,156.8,142.9,137.7,137.2,132.7,132.6,128.8,127.7,127.0,125.3$, $114.9,114.7,111.6,110.5,78.3,55.8,55.6,37.7,24.4,13.9$. HR-ESI-MS calcd. for $\mathrm{C}_{25} \mathrm{H}_{26} \mathrm{NO}_{4}[\mathrm{M}+\mathrm{H}]^{+}:$404.1856, found: 404.1859. HPLC (Daicel Chiralpak OZ-H (25 $\mathrm{cm}$ ), Hexanes $/ \mathrm{IPA}=60 / 40$, flow rate $=0.5 \mathrm{~mL} / \mathrm{min}, \mathrm{I}=280 \mathrm{~nm}) \mathrm{t}_{\mathrm{R}}=21.11 \mathrm{~min}$ (major), $26.16 \mathrm{~min}$ (minor).

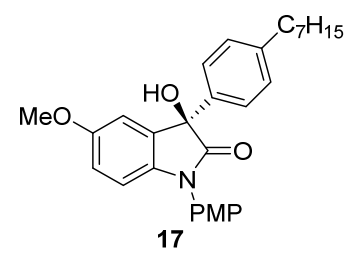

(R)-3-(4-Heptylphenyl)-3-hydroxy-5-methoxy-1-(4-methoxyphenyl)indolin-2-one (17): Prepared according to general procedures. Colorless oil (22.1 mg, 48\% yield, 95\% ee). Flash column chromatography conditions: petroleum ether/ethyl acetate $=2.5 / 1$. $[\alpha]_{\mathrm{D}}{ }^{20}=+51.4\left(c 1.0, \mathrm{CHCl}_{3}\right) ;{ }^{1} \mathrm{H} \mathrm{NMR}\left(600 \mathrm{MHz}, \mathrm{CDCl}_{3}\right) \delta=7.37(\mathrm{~d}, J=8.2,2 \mathrm{H})$, 
$7.34(\mathrm{~d}, J=8.7,2 \mathrm{H}), 7.16(\mathrm{~d}, J=8.2,2 \mathrm{H}), 7.01(\mathrm{~d}, J=8.8,2 \mathrm{H}), 6.95(\mathrm{~d}, J=2.5,1 \mathrm{H})$, $6.78(\mathrm{dd}, J=8.6,2.5,1 \mathrm{H}), 6.74(\mathrm{~d}, J=8.6,1 \mathrm{H}), 3.99(\mathrm{~s}, 1 \mathrm{H}), 3.84(\mathrm{~s}, 3 \mathrm{H}), 3.74(\mathrm{~s}, 3 \mathrm{H})$, $2.66-2.55(\mathrm{~m}, 2 \mathrm{H}), 1.66-1.54(\mathrm{~m}, 2 \mathrm{H}), 1.38-1.20(\mathrm{~m}, 8 \mathrm{H}), 0.89(\mathrm{t}, J=7.0,3 \mathrm{H}) .{ }^{13} \mathrm{C}$ NMR (150 MHz, $\left.\mathrm{CDCl}_{3}\right) \delta=177.2,159.2,156.7,143.2,137.7,137.2,132.7,128.7$, 127.7, 127.0, 125.3, 114.9, 114.7, 111.6, 110.5, 78.3, 55.8, 55.6, 35.7, 31.8, 31.4, 29.3, 29.2, 22.7, 14.1. HR-ESI-MS calcd. for $\mathrm{C}_{29} \mathrm{H}_{34} \mathrm{NO}_{4}[\mathrm{M}+\mathrm{H}]^{+}:$460.2482, found: 460.2482. HPLC (Daicel Chiralpak OZ-H (25 cm), Hexanes $/ \mathrm{IPA}=60 / 40$, flow rate $=$ $0.5 \mathrm{~mL} / \mathrm{min}, \mathrm{I}=280 \mathrm{~nm}$ ) $\mathrm{t}_{\mathrm{R}}=18.01 \mathrm{~min}$ (major), $21.12 \min$ (minor).

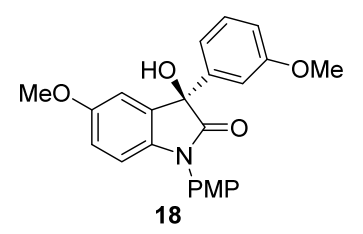

(R)-3-Hydroxy-5-methoxy-3-(3-methoxyphenyl)-1-(4-methoxyphenyl)indolin-2one (18) : Prepared according to general procedures. Colorless oil (34.1 mg, 87\% yield, 95\% ee). Flash column chromatography conditions: petroleum ether/ethyl acetate $=$ 2.5/1. $[\alpha]_{\mathrm{D}}{ }^{20}=+98.6\left(c 1.0, \mathrm{CHCl}_{3}\right) ;{ }^{1} \mathrm{H} \mathrm{NMR}(600 \mathrm{MHz}, \mathrm{MeOD}) \delta=7.86(\mathrm{~s}, 1 \mathrm{H}), 7.29$ $(\mathrm{d}, J=8.8,2 \mathrm{H}), 7.20(\mathrm{t}, J=8.0,1 \mathrm{H}), 7.16(\mathrm{~s}, 1 \mathrm{H}), 7.05(\mathrm{~d}, J=8.8,2 \mathrm{H}), 6.87(\mathrm{~d}, J=$ $7.2,2 \mathrm{H}), 6.86-6.80(\mathrm{~m}, 2 \mathrm{H}), 6.70(\mathrm{~d}, J=8.6,1 \mathrm{H}), 3.80(\mathrm{~s}, 3 \mathrm{H}), 3.75(\mathrm{~s}, 3 \mathrm{H}), 3.69(\mathrm{~s}$, $3 \mathrm{H}) .{ }^{13} \mathrm{C}$ NMR (150 MHz, MeOD) $\delta$ 177.6, 159.9, 159.5, 157.0, 142.1, 137.2, 133.8, 129.2 , 127.7, 126.9, 117.5, 114.7, 114.1, 113.1, 111.4, 111.3, 110.2, 78.1, 78.0, 54.9, 54.7, 54.4. HR-ESI-MS calcd. for $\mathrm{C}_{23} \mathrm{H}_{22} \mathrm{NO}_{5}[\mathrm{M}+\mathrm{H}]^{+}:$392.1492, found: 392.1494 . HPLC (Daicel Chiralpak AD-H $(25 \mathrm{~cm})$, Hexanes $/ \mathrm{IPA}=50 / 50$ flow rate $=0.5 \mathrm{~mL} / \mathrm{min}$, $\mathrm{I}=280 \mathrm{~nm}) \mathrm{t}_{\mathrm{R}}=49.40 \mathrm{~min}$ (major), $27.96 \mathrm{~min}$ (minor).

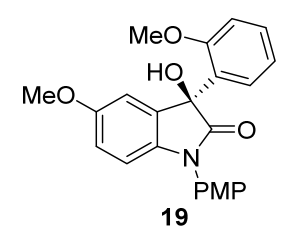

(S)-3-Hydroxy-5-methoxy-3-(2-methoxyphenyl)-1-(4-methoxyphenyl)indolin-2one (19): Prepared according to general procedures. Colorless oil (19.9 mg, 51\% yield, 
90\% ee). Flash column chromatography conditions: petroleum ether/ethyl acetate $=$ 2.5/1. $[\alpha]_{\mathrm{D}}{ }^{20}=+18.3\left(c 1.0, \mathrm{CHCl}_{3}\right) ;{ }^{1} \mathrm{H} \mathrm{NMR}\left(600 \mathrm{MHz}, \mathrm{CDCl}_{3}\right) \delta=7.82(\mathrm{~d}, J=7.5$, 1H), $7.41(\mathrm{~d}, J=8.7,2 \mathrm{H}), 7.31(\mathrm{t}, J=7.8,1 \mathrm{H}), 7.08-7.04(\mathrm{~m}, 3 \mathrm{H}), 6.84(\mathrm{~d}, J=8.2$, $1 \mathrm{H}), 6.75(\mathrm{~s}, 3 \mathrm{H}), 3.86(\mathrm{~s}, 3 \mathrm{H}), 3.71(\mathrm{~s}, 3 \mathrm{H}), 3.66(\mathrm{~s}, 3 \mathrm{H}) .{ }^{13} \mathrm{C} \mathrm{NMR}\left(150 \mathrm{MHz}, \mathrm{CDCl}_{3}\right)$ $\delta 176.9,159.0,156.4,156.1,138.1,131.3,129.6,129.2,127.5,127.4,126.8,121.1$, $114.9,114.4,111.8,111.3,109.8,76.7,56.0,55.8,55.6$. HR-ESI-MS calcd. for $\mathrm{C}_{23} \mathrm{H}_{22} \mathrm{NO} 5[\mathrm{M}+\mathrm{H}]^{+}:$392.1492, found: 392.1488. HPLC (Daicel Chiralpak AD-H (25 $\mathrm{cm})$, Hexanes $/ \mathrm{IPA}=50 / 50$ flow rate $=0.5 \mathrm{~mL} / \mathrm{min}, \mathrm{I}=280 \mathrm{~nm}) \mathrm{t}_{\mathrm{R}}=103.81 \mathrm{~min}$ (major), $49.73 \min$ (minor).

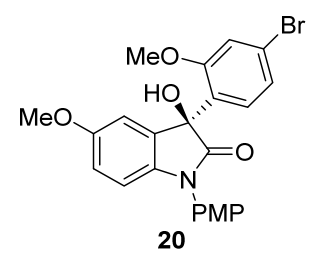

(S)-3-(4-Bromo-2-methoxyphenyl)-3-hydroxy-5-methoxy-1-(4-methoxyphenyl)

indolin-2-one (20): Prepared according to general procedures. Blue oil (29.1 mg, 62\% yield, 92\% ee). Flash column chromatography conditions: petroleum ether/ethyl acetate $=2.5 / 1 .[\alpha]_{\mathrm{D}}{ }^{20}=+18.4\left(c 1.0, \mathrm{CHCl}_{3}\right) ;{ }^{1} \mathrm{H} \mathrm{NMR}\left(600 \mathrm{MHz}, \mathrm{CDCl}_{3}\right) \delta=7.72(\mathrm{~d}, J=8.3$, $1 \mathrm{H}), 7.36(\mathrm{~d}, J=8.7,2 \mathrm{H}), 7.16(\mathrm{~d}, J=8.2,1 \mathrm{H}), 7.04(\mathrm{~d}, J=8.7,2 \mathrm{H}), 6.93(\mathrm{~s}, 1 \mathrm{H}), 6.77$ $-6.70(\mathrm{~m}, 2 \mathrm{H}), 6.65(\mathrm{~s}, 1 \mathrm{H}), 4.36(\mathrm{~s}, 1 \mathrm{H}), 3.85(\mathrm{~s}, 3 \mathrm{H}), 3.67(\mathrm{~s}, 3 \mathrm{H}), 3.59(\mathrm{~s}, 3 \mathrm{H}) .{ }^{13} \mathrm{C}$ NMR $\left(150 \mathrm{MHz}, \mathrm{CDCl}_{3}\right) \delta 176.6,159.0,156.4,156.3,138.0,131.2,128.5,128.3,127.4$, 124.0, 122.8, 114.9, 114.4, 111.1, 109.9, 76.2, 56.2, 55.8, 55.6. HR-ESI-MS calcd. for $\mathrm{C}_{23} \mathrm{H}_{20} \mathrm{NO}_{5} \mathrm{BrNa}[\mathrm{M}+\mathrm{Na}]^{+}:$492.0417, found: 492.0418. HPLC (Daicel Chiralpak AD$\mathrm{H}(25 \mathrm{~cm})$, Hexanes $/ \mathrm{IPA}=50 / 50$ flow rate $=0.5 \mathrm{~mL} / \mathrm{min}, \mathrm{I}=280 \mathrm{~nm}) \mathrm{t}_{\mathrm{R}}=121.54 \mathrm{~min}$ (major), $67.01 \mathrm{~min}$ (minor).

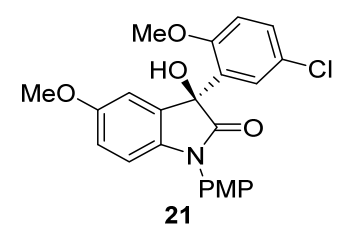

(S)-3-(5-Chloro-2-methoxyphenyl)-3-hydroxy-5-methoxy-1-(4-methoxyphenyl) 
indolin-2-one (21): Prepared according to general procedures. White powder (33.2 mg, $78 \%$ yield, $80 \%$ ee). mp: $229.2-231.9^{\circ} \mathrm{C}$. Flash column chromatography conditions: petroleum ether/ethyl acetate $=2.5 / 1 .[\alpha] \mathrm{D}^{20}=+73.3\left(c 1.0, \mathrm{CHCl}_{3}\right) ;{ }^{1} \mathrm{H} \mathrm{NMR}(600 \mathrm{MHz}$, DMSO) $\delta=7.86(\mathrm{~s}, 1 \mathrm{H}), 7.38-7.35(\mathrm{~m}, 3 \mathrm{H}), 7.15(\mathrm{~d}, J=6.5,2 \mathrm{H}), 7.10-6.93(\mathrm{~m}$, 2H), $6.79(\mathrm{~d}, J=8.5,1 \mathrm{H}), 6.65(\mathrm{dd}, J=8.5,2.3,1 \mathrm{H}), 6.56(\mathrm{~s}, 1 \mathrm{H}), 3.83(\mathrm{~s}, 3 \mathrm{H}), 3.64$ (s, 3H), $3.50(\mathrm{~s}, 3 \mathrm{H}) .{ }^{13} \mathrm{C}$ NMR (150 MHz, DMSO) $\delta=175.7,158.9,156.0,154.8$, $138.3,132.9,132.5,129.1,128.0,127.9,127.2,124.9,115.4,114.0,113.8,111.2,109.5$, 75.1, 56.7, 56.0, 55.9. HR-ESI-MS calcd. for $\mathrm{C}_{23} \mathrm{H}_{20} \mathrm{NO}_{5} \mathrm{CINa}[\mathrm{M}+\mathrm{Na}]^{+}$: 448.0922, found: 448.0923. HPLC (Daicel Chiralpak AD-H $(25 \mathrm{~cm})$, Hexanes/IPA = 50/50 flow rate $=0.5 \mathrm{~mL} / \mathrm{min}, \mathrm{I}=280 \mathrm{~nm}$ ) $\mathrm{t}_{\mathrm{R}}=79.97 \mathrm{~min}$ (major), $25.55 \mathrm{~min}$ (minor).

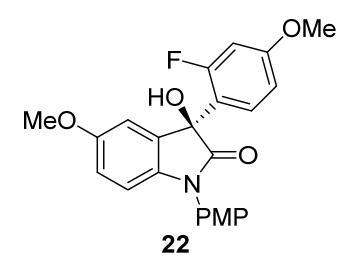

(S)-3-(2-Fluoro-4-methoxyphenyl)-3-hydroxy-5-methoxy-1-(4-methoxyphenyl)

indolin-2-one (22): Prepared according to general procedures. Colorless oil (27.1 mg, $66 \%$ yield, $72 \%$ ee). Flash column chromatography conditions: petroleum ether/ethyl acetate $=2 / 1 .[\alpha]_{\mathrm{D}}{ }^{20}=+48.6\left(c 1.0, \mathrm{CHCl}_{3}\right) ;{ }^{1} \mathrm{H} \mathrm{NMR}\left(600 \mathrm{MHz}, \mathrm{CDCl}_{3}\right) \delta=7.78(\mathrm{t}, J$ $=8.9,1 \mathrm{H}), 7.37(\mathrm{~d}, J=8.8,2 \mathrm{H}), 7.03(\mathrm{~d}, J=8.8,2 \mathrm{H}), 6.75(\mathrm{ddd}, J=13.4,8.8,2.3,3 \mathrm{H})$, $6.69(\mathrm{~d}, J=8.5,1 \mathrm{H}), 6.52(\mathrm{dd}, J=12.8,2.3,1 \mathrm{H}), 3.97(\mathrm{~s}, 1 \mathrm{H}), 3.85(\mathrm{~s}, 3 \mathrm{H}), 3.78(\mathrm{~s}$, 3H), $3.70(\mathrm{~s}, 3 \mathrm{H}) .{ }^{13} \mathrm{C}$ NMR $\left(150 \mathrm{MHz}, \mathrm{CDCl}_{3}\right) \delta 176.4,161.1,161.0,160.5,159.3$, $158.9,156.6,137.6,131.2,127.9,127.8,127.0,120.1,120.0,115.0,114.7,111.3,110.4$, 109.7, 102.3, 102.1, 75.6, 55.8, 55.6, 55.6. $\left.{ }^{19} \mathrm{~F} \mathrm{NMR} \mathrm{(565} \mathrm{MHz,} \mathrm{CDCl}_{3}\right) \delta=-112.3$. HR-ESI-MS calcd. for $\mathrm{C}_{23} \mathrm{H}_{20} \mathrm{NO}_{5} \mathrm{FNa}[\mathrm{M}+\mathrm{Na}]^{+}:$432.1218, found: 432.1219. HPLC (Daicel Chiralpak AS-H $(25 \mathrm{~cm})$, Hexanes/IPA $=50 / 50$ flow rate $=0.5 \mathrm{~mL} / \mathrm{min}, \mathrm{I}=280$ $\mathrm{nm}) \mathrm{t}_{\mathrm{R}}=18.31 \mathrm{~min}$ (major), $39.99 \min ($ minor). 


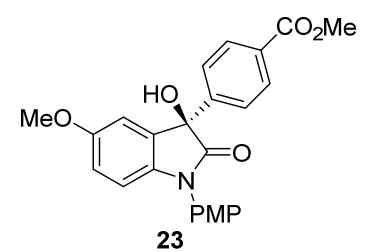

Methyl (R)-4-(3-hydroxy-5-methoxy-1-(4-methoxyphenyl)-2-oxoindolin-3yl)benzoate (23): Prepared according to general procedures. Colorless oil (31.9 mg, 76\% yield, 93\% ee). Flash column chromatography conditions: petroleum ether/ethyl acetate $=2.5 / 1 .[\alpha]_{\mathrm{D}}{ }^{20}=+69.1\left(c 1.0, \mathrm{CHCl}_{3}\right) ;{ }^{1} \mathrm{H} \mathrm{NMR}\left(600 \mathrm{MHz}, \mathrm{CDCl}_{3}\right) \delta 7.94(\mathrm{~d}, J=8.4$ Hz, 2H), $7.48(\mathrm{~d}, J=8.4 \mathrm{~Hz}, 2 \mathrm{H}), 7.28(\mathrm{~d}, J=8.8 \mathrm{~Hz}, 2 \mathrm{H}), 6.98(\mathrm{~d}, J=8.9 \mathrm{~Hz}, 2 \mathrm{H})$, $6.83(\mathrm{~d}, J=2.4 \mathrm{~Hz}, 1 \mathrm{H}), 6.76(\mathrm{~d}, J=2.5 \mathrm{~Hz}, 1 \mathrm{H}), 6.72(\mathrm{~d}, J=8.6 \mathrm{~Hz}, 1 \mathrm{H}), 3.88(\mathrm{~s}, 3 \mathrm{H})$, $3.82(\mathrm{~s}, 3 \mathrm{H}), 3.68(\mathrm{~s}, 3 \mathrm{H}) .{ }^{13} \mathrm{C} \mathrm{NMR}\left(150 \mathrm{MHz}, \mathrm{CDCl}_{3}\right) \delta 176.6,166.8,159.2,156.9$, $145.5,137.0,132.5,129.9,127.6,126.7,125.5,114.98,114.96,111.4,110.7,78.4,55.8$, 55.6, 52.2. HR-ESI-MS calcd. for $\mathrm{C}_{24} \mathrm{H}_{22} \mathrm{NO}_{6}[\mathrm{M}+\mathrm{H}]^{+}$: 420.1442 , found: 420.1444 . HPLC (Daicel Chiralpak OZ-H $(25 \mathrm{~cm})$, Hexanes $/ \mathrm{IPA}=50 / 50$ flow rate $=0.5 \mathrm{~mL} / \mathrm{min}$, $\mathrm{I}=280 \mathrm{~nm}$ ) $\mathrm{t}_{\mathrm{R}}=24.47 \mathrm{~min}$ (major), $49.12 \mathrm{~min}$ (minor).

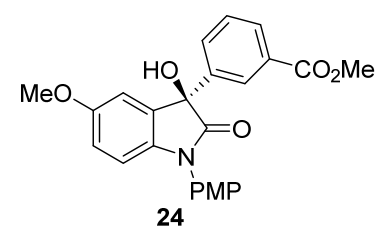

Methyl

(R)-4-(3-hydroxy-5-methoxy-1-(4-methoxyphenyl)-2-oxoindolin-3-

yl)benzoate (24): Prepared according to general procedures. Light blue oil (30.2 mg, 72\% yield, 95\% ee). Flash column chromatography conditions: petroleum ether/ethyl acetate $=2.5 / 1 .[\alpha]_{\mathrm{D}}{ }^{20}=+37.6\left(c 1.0, \mathrm{CHCl}_{3}\right) ;{ }^{1} \mathrm{H} \mathrm{NMR}\left(600 \mathrm{MHz}, \mathrm{CDCl}_{3}\right) \delta=8.12(\mathrm{~s}$, 1H), 7.95 (d, $J=7.8,1 \mathrm{H}), 7.63$ (d, $J=7.9,1 \mathrm{H}), 7.37$ (t, $J=7.8,1 \mathrm{H}), 7.30$ (d, $J=8.9$, 2H), $6.99(\mathrm{~d}, J=8.9,2 \mathrm{H}), 6.85(\mathrm{~d}, J=2.5,1 \mathrm{H}), 6.77(\mathrm{dd}, J=8.6,2.5,1 \mathrm{H}), 6.73(\mathrm{~d}, J=$ 8.6, 1H), $4.60(\mathrm{~s}, 1 \mathrm{H}), 3.86(\mathrm{~s}, 3 \mathrm{H}), 3.82(\mathrm{~s}, 3 \mathrm{H}), 3.69$ (s, 3H). ${ }^{13} \mathrm{C}$ NMR (150 MHz, $\left.\mathrm{CDCl}_{3}\right) \delta 176.7,166.8,159.2,156.9,141.1,137.1,132.4,130.5,130.0,129.5,128.8$, 127.7, 126.7, 126.5, 115.0, 111.5, 110.8, 78.2, 55.8, 55.6, 52.2. HR-ESI-MS calcd. for $\mathrm{C}_{24} \mathrm{H}_{22} \mathrm{NO}_{6}[\mathrm{M}+\mathrm{H}]^{+}:$420.1442, found: 420.1443. HPLC (Daicel Chiralpak AS-H (25 $\mathrm{cm}$ ), Hexanes $/ \mathrm{IPA}=50 / 50$ flow rate $=0.5 \mathrm{~mL} / \mathrm{min}, \mathrm{I}=280 \mathrm{~nm}$ ) $\mathrm{t}_{\mathrm{R}}=15.63 \mathrm{~min}$ (major), 
$34.46 \min$ (minor).

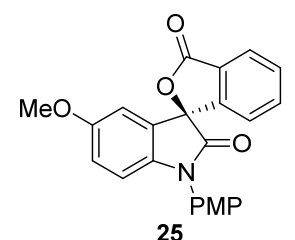

(R)-5-Methoxy-1-(4-methoxyphenyl)-3' H-spiro[indoline-3,1'-isobenzofuran]-2,3'dione (25): Prepared according to general procedures. White powder $(32.9 \mathrm{mg}, 85 \%$ yield, $40 \%$ ee). mp: $151.5-153.1{ }^{\circ} \mathrm{C}$. Flash column chromatography conditions: petroleum ether/ethyl acetate $=2.5 / 1 .[\alpha]_{\mathrm{D}}{ }^{20}=-4.1\left(c 1.0, \mathrm{CHCl}_{3}\right) ;{ }^{1} \mathrm{H}$ NMR $(600 \mathrm{MHz}$, $\left.\mathrm{CDCl}_{3}\right) \delta 8.00(\mathrm{~d}, J=7.6 \mathrm{~Hz}, 1 \mathrm{H}), 7.65(\mathrm{t}, J=7.5 \mathrm{~Hz}, 1 \mathrm{H}), 7.61(\mathrm{t}, J=7.5 \mathrm{~Hz}, 1 \mathrm{H})$, $7.35(\mathrm{~d}, J=8.8 \mathrm{~Hz}, 2 \mathrm{H}), 7.23(\mathrm{~d}, J=7.6 \mathrm{~Hz}, 1 \mathrm{H}), 7.02(\mathrm{~d}, J=8.8 \mathrm{~Hz}, 2 \mathrm{H}), 6.88(\mathrm{dd}, J$ $=8.7,2.5 \mathrm{~Hz}, 1 \mathrm{H}), 6.82(\mathrm{~d}, J=8.7 \mathrm{~Hz}, 1 \mathrm{H}), 6.63(\mathrm{~d}, J=2.5 \mathrm{~Hz}, 1 \mathrm{H}), 3.83(\mathrm{~s}, 3 \mathrm{H}), 3.69$ (s, 3H). ${ }^{13} \mathrm{C}$ NMR $\left(150 \mathrm{MHz}, \mathrm{CDCl}_{3}\right) \delta 170.5,169.6,159.5,157.0,147.1,138.2,135.0$, $130.5,127.6,126.4,126.1,125.8,125.6,121.9,116.7,115.1,111.5,111.2,84.8,55.9$, 55.6. HR-ESI-MS calcd. for $\mathrm{C}_{23} \mathrm{H}_{18} \mathrm{NO}_{5}[\mathrm{M}+\mathrm{H}]^{+}: 388.1179$, found: 388.1183. HPLC (Daicel Chiralpak OZ-H (25 cm), Hexanes $/ \mathrm{IPA}=50 / 50$ flow rate $=0.5 \mathrm{~mL} / \mathrm{min}, \mathrm{I}=280$ $\mathrm{nm}) \mathrm{t}_{\mathrm{R}}=42.01 \mathrm{~min}$ (major), $27.88 \mathrm{~min}$ (minor).

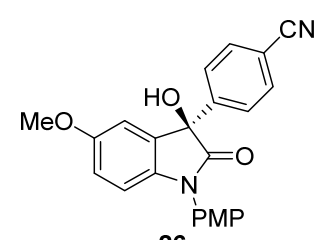

26

(R)-4-(3-Hydroxy-5-methoxy-1-(4-methoxyphenyl)-2-oxoindolin-3-yl)benzonitrile (26): Prepared according to general procedures. Colorless oil (27.4 mg, 71\% yield, 77\% ee). Flash column chromatography conditions: petroleum ether/ethyl acetate $=2.5 / 1$. $[\alpha]_{\mathrm{D}^{20}}=+36.9\left(c 1.0, \mathrm{CHCl}_{3}\right) ;{ }^{1} \mathrm{H} \mathrm{NMR}\left(600 \mathrm{MHz}, \mathrm{CDCl}_{3}\right) \delta=7.58(\mathrm{~d}, J=8.4,2 \mathrm{H})$, $7.52(\mathrm{~d}, J=8.4,2 \mathrm{H}), 7.26(\mathrm{~d}, J=8.8,2 \mathrm{H}), 6.99(\mathrm{~d}, J=8.8,2 \mathrm{H}), 6.83-6.77(\mathrm{~m}, 2 \mathrm{H})$, $6.74(\mathrm{~d}, J=8.4,1 \mathrm{H}), 4.77(\mathrm{~s}, 1 \mathrm{H}), 3.84(\mathrm{~s}, 3 \mathrm{H}), 3.71(\mathrm{~s}, 3 \mathrm{H}) .{ }^{13} \mathrm{C}$ NMR $(150 \mathrm{MHz}$, $\left.\mathrm{CDCl}_{3}\right) \delta=176.2,159.4,157.0,145.6,137.0,132.4,131.9,127.6,126.4,126.3,118.6$, 115.1, 115.0, 112.0, 111.5, 110.9, 78.2, 55.8, 55.6. HR-ESI-MS calcd. for 
$\mathrm{C}_{23} \mathrm{H}_{18} \mathrm{~N}_{2} \mathrm{O}_{4} \mathrm{Na}[\mathrm{M}+\mathrm{Na}]^{+}:$409.1159, found: 409.1158. HPLC (Daicel Chiralpak OZ-H $(25 \mathrm{~cm})$, Hexanes $/ \mathrm{IPA}=50 / 50$ flow rate $=0.5 \mathrm{~mL} / \mathrm{min}, \mathrm{I}=280 \mathrm{~nm}) \mathrm{t}_{\mathrm{R}}=15.24 \mathrm{~min}$ (major), $20.48 \mathrm{~min}$ (minor).

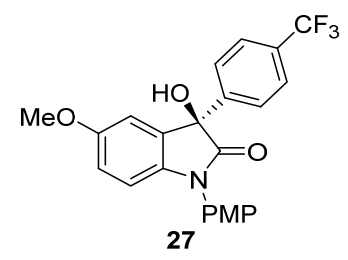

(R)-3-Hydroxy-5-methoxy-1-(4-methoxyphenyl)-3-(4-(trifluoromethyl)phenyl) indolin-2-one (27): Prepared according to general procedures. Colorless oil (37.8 mg, $88 \%$ yield, $90 \%$ ee). Flash column chromatography conditions: petroleum ether/ethyl acetate $=2.5 / 1$. $[\alpha]_{\mathrm{D}}^{20}=+23.5\left(c 1.0, \mathrm{CHCl}_{3}\right) ;{ }^{1} \mathrm{H} \mathrm{NMR}\left(600 \mathrm{MHz}, \mathrm{CDCl}_{3}\right) \delta=7.56(\mathrm{q}, J=8.6,4 \mathrm{H})$, $7.29(\mathrm{~d}, J=8.8,2 \mathrm{H}), 7.00(\mathrm{~d}, J=8.9,2 \mathrm{H}), 6.86(\mathrm{~d}, J=2.4,1 \mathrm{H}), 6.80(\mathrm{dd}, J=8.6,2.5$, 1H), $6.75(\mathrm{~d}, J=8.6,1 \mathrm{H}), 4.91(\mathrm{~s}, 1 \mathrm{H}), 3.84(\mathrm{~s}, 3 \mathrm{H}), 3.71(\mathrm{~s}, 3 \mathrm{H}) .{ }^{13} \mathrm{C} \mathrm{NMR}(150 \mathrm{MHz}$, $\left.\mathrm{CDCl}_{3}\right) \delta=176.7,159.3,157.0,144.5,137.0,132.3,130.6,130.4,130.2,130.0,127.6$, $126.8,126.6,126.0,125.6,125.6,125.5,125.0$, 123.2, 121.4, 115.02, 114.97, 111.5, 110.8, 78.3, 55.8, 55.5. ${ }^{19} \mathrm{~F} \mathrm{NMR}\left(565 \mathrm{MHz}, \mathrm{CDCl}_{3}\right) \delta=-62.6$. HR-ESI-MS calcd. for $\mathrm{C}_{23} \mathrm{H}_{18} \mathrm{NO}_{4} \mathrm{~F}_{3} \mathrm{Na}[\mathrm{M}+\mathrm{Na}]^{+}: 452.1080$, found: 452.1080. HPLC (Daicel Chiralpak OZ$\mathrm{H}(25 \mathrm{~cm})$, Hexanes $/ \mathrm{IPA}=50 / 50$ flow rate $=0.5 \mathrm{~mL} / \mathrm{min}, \mathrm{I}=280 \mathrm{~nm}) \mathrm{t}_{\mathrm{R}}=8.62 \mathrm{~min}$ (major), $20.63 \min$ (minor).

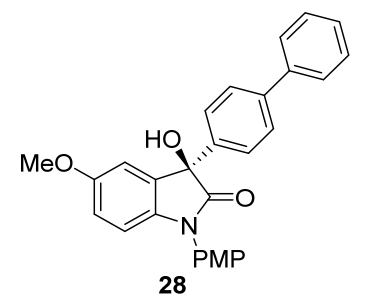

(R)-3-([1,1'-Biphenyl]-4-yl)-3-hydroxy-5-methoxy-1-(4-methoxyphenyl)indolin-2one (28): Prepared according to general procedures. Colorless oil (35.8 mg, 82\% yield, 90\% ee). Flash column chromatography conditions: petroleum ether/ethyl acetate $=2 / 1$. $[\alpha]_{\mathrm{D}}{ }^{20}=+83.9\left(c 1.0, \mathrm{CHCl}_{3}\right) ;{ }^{1} \mathrm{H}$ NMR $\left(600 \mathrm{MHz}, \mathrm{CDCl}_{3}\right) \delta=7.56(\mathrm{q}, J=8.4,6 \mathrm{H})$, $7.43(\mathrm{t}, J=7.6,2 \mathrm{H}), 7.35(\mathrm{t}, J=7.9,3 \mathrm{H}), 7.02(\mathrm{~d}, J=8.8,2 \mathrm{H}), 6.99(\mathrm{~d}, J=2.4,1 \mathrm{H})$, 
$6.81(\mathrm{dd}, J=8.7,2.4,1 \mathrm{H}), 6.77(\mathrm{~d}, J=8.7,1 \mathrm{H}), 4.29(\mathrm{~s}, 1 \mathrm{H}), 3.84(\mathrm{~s}, 3 \mathrm{H}), 3.75(\mathrm{~s}$, $3 \mathrm{H}) .{ }^{13} \mathrm{C} \mathrm{NMR}\left(150 \mathrm{MHz}, \mathrm{CDCl}_{3}\right) \delta=177.1,159.2,156.9,141.2,140.6,139.5,137.2$, $132.6,128.8,127.7,127.4,127.1,126.9,125.9,115.0,114.9,111.6,110.7,78.4,55.9$, 55.6. HR-ESI-MS calcd. for $\mathrm{C}_{28} \mathrm{H}_{23} \mathrm{NO}_{4} \mathrm{Na}[\mathrm{M}+\mathrm{Na}]^{+}$: 460.1519 , found: 460.1514 . HPLC (Daicel Chiralpak OZ-H $(25 \mathrm{~cm})$, Hexanes $/ \mathrm{IPA}=50 / 50$ flow rate $=0.5 \mathrm{~mL} / \mathrm{min}$, $\mathrm{I}=280 \mathrm{~nm}) \mathrm{t}_{\mathrm{R}}=15.04 \mathrm{~min}$ (major), $26.23 \mathrm{~min}$ (minor).

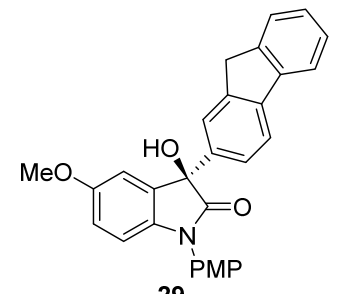

29

(R)-3-(9H-Fluoren-2-yl)-3-hydroxy-5-methoxy-1-(4-methoxyphenyl)indolin-2-one (29): Prepared according to general procedures. Colorless oil (17.9 mg, 40\% yield, 94\% ee). Flash column chromatography conditions: petroleum ether/ethyl acetate $=2.5 / 1$. $[\alpha]_{\mathrm{D}}{ }^{20}=+22.1\left(c 1.0, \mathrm{CHCl}_{3}\right) ;{ }^{1} \mathrm{H} \mathrm{NMR}\left(600 \mathrm{MHz}, \mathrm{CDCl}_{3}\right) \delta=7.78-7.64(\mathrm{~m}, 3 \mathrm{H})$, $7.51(\mathrm{~d}, J=7.4,1 \mathrm{H}), 7.45(\mathrm{~d}, J=7.9,1 \mathrm{H}), 7.36(\mathrm{t}, J=8.8,3 \mathrm{H}), 7.30(\mathrm{t}, J=7.3,1 \mathrm{H})$, $7.02(\mathrm{~d}, J=8.8,2 \mathrm{H}), 6.97(\mathrm{~d}, J=2.1,1 \mathrm{H}), 6.81-6.77(\mathrm{~m}, 2 \mathrm{H}), 4.19(\mathrm{~s}, 1 \mathrm{H}), 3.83(\mathrm{~s}$, 5H), $3.74(\mathrm{~s}, 3 \mathrm{H}) .{ }^{13} \mathrm{C} \mathrm{NMR}\left(150 \mathrm{MHz}, \mathrm{CDCl}_{3}\right) \delta 177.3,159.2,156.8,143.7,143.5$, 142.0, 141.2, 139.0, 137.2, 132.8, 127.7, 126.9, 126.8, 125.1, 124.1, 122.1, 120.1, 120.0, 115.0, 114.8, 111.6, 110.6, 78.7, 55.9, 55.6, 37.0. HR-ESI-MS calcd. for $\mathrm{C}_{29} \mathrm{H}_{23} \mathrm{NO}_{4} \mathrm{Na}$ $[\mathrm{M}+\mathrm{Na}]^{+}:$472.1519, found: 472.1523. HPLC (Daicel Chiralpak OZ-H $(25 \mathrm{~cm})$, Hexanes $/ \mathrm{IPA}=50 / 50$ flow rate $=0.5 \mathrm{~mL} / \mathrm{min}, \mathrm{I}=280 \mathrm{~nm}$ ) $\mathrm{t}_{\mathrm{R}}=19.45 \mathrm{~min}$ (major), $32.45 \min$ (minor).

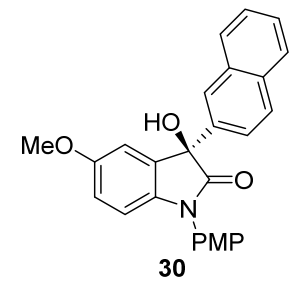

(R)-3-Hydroxy-5-methoxy-1-(4-methoxyphenyl)-3-(naphthalen-2-yl)indolin-2-one (30): Prepared according to general procedures. Light blue oil (32.9 mg, 80\% yield, 94\% 
ee). Flash column chromatography conditions: petroleum ether/ethyl acetate $=2.5 / 1$. $[\alpha]_{\mathrm{D}^{20}}=+76.4\left(c 1.0, \mathrm{CHCl}_{3}\right) ;{ }^{1} \mathrm{H} \mathrm{NMR}\left(600 \mathrm{MHz}, \mathrm{CDCl}_{3}\right) \delta=8.03(\mathrm{~s}, 1 \mathrm{H}), 7.80(\mathrm{~d}, J$ $=7.1,2 \mathrm{H}), 7.76(\mathrm{~d}, J=8.6,1 \mathrm{H}), 7.53-7.41(\mathrm{~m}, 3 \mathrm{H}), 7.36(\mathrm{~d}, J=8.8,2 \mathrm{H}), 7.01(\mathrm{~d}, J$ $=8.8,2 \mathrm{H}), 6.96(\mathrm{~s}, 1 \mathrm{H}), 6.82-6.78((\mathrm{~m}, 2 \mathrm{H}), 4.65(\mathrm{~s}, 1 \mathrm{H}), 3.83(\mathrm{~s}, 3 \mathrm{H}), 3.70(\mathrm{~s}, 3 \mathrm{H})$. ${ }^{13} \mathrm{C} \mathrm{NMR}\left(150 \mathrm{MHz}, \mathrm{CDCl}_{3}\right) \delta 177.2,159.2,156.9,137.9,137.2,133.17,133.15,132.8$, $128.7,128.4,127.71,127.65,126.9,126.4,126.3,124.4,123.3,115.0,111.6,110.7$, 78.7, 55.8, 55.6. HR-ESI-MS calcd. for $\mathrm{C}_{26} \mathrm{H}_{21} \mathrm{NO}_{4} \mathrm{Na}[\mathrm{M}+\mathrm{Na}]^{+}: 434.1363$, found: 434.1365. HPLC (Daicel Chiralpak OZ-H (25 cm), Hexanes/IPA = 50/50 flow rate = $0.5 \mathrm{~mL} / \mathrm{min}, \mathrm{I}=320 \mathrm{~nm}$ ) $\mathrm{t}_{\mathrm{R}}=18.68 \mathrm{~min}$ (major), $27.02 \mathrm{~min}$ (minor).

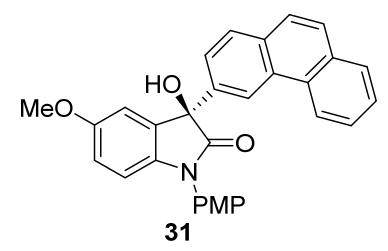

\section{(R)-3-Hydroxy-5-methoxy-1-(4-methoxyphenyl)-3-(phenanthren-3-yl)indolin-2-}

one (31): Prepared according to general procedures. yellow oil $(23.9 \mathrm{mg}, 52 \%$ yield, 90\% ee). Flash column chromatography conditions: petroleum ether/ethyl acetate $=$ 2.5/1. $[\alpha]_{\mathrm{D}}{ }^{20}=+7.7\left(c 1.0, \mathrm{CHCl}_{3}\right) ;{ }^{1} \mathrm{H} \mathrm{NMR}\left(600 \mathrm{MHz}, \mathrm{CDCl}_{3}\right) \delta=8.55(\mathrm{~d}, J=8.2$, $2 \mathrm{H}), 8.03(\mathrm{~s}, 1 \mathrm{H}), 7.86(\mathrm{~d}, J=7.3,1 \mathrm{H}), 7.72-7.55(\mathrm{~m}, 5 \mathrm{H}), 7.39(\mathrm{~d}, J=7.2,2 \mathrm{H}), 7.01$ $(\mathrm{d}, J=9.3,3 \mathrm{H}), 6.86-6.76(\mathrm{~m}, 2 \mathrm{H}), 4.82(\mathrm{~s}, 1 \mathrm{H}), 3.81(\mathrm{~s}, 3 \mathrm{H}), 3.70(\mathrm{~s}, 3 \mathrm{H}) .{ }^{13} \mathrm{C} \mathrm{NMR}$ $\left(150 \mathrm{MHz} \mathrm{CDCl}_{3}\right) \delta=177.3,159.2,156.9,138.7,137.2,132.8,132.2,132.0,130.1$, 130.0, 128.6, 127.7, 127.4, 127.1, 126.9, 126.8, 126.7, 125.2, 123.8, 123.4, 122.8, 115.0, 111.6, 110.7, 78.7, 55.8, 55.6. HR-ESI-MS calcd. for $\mathrm{C}_{30} \mathrm{H}_{23} \mathrm{NO}_{4} \mathrm{Na}[\mathrm{M}+\mathrm{Na}]^{+}$: 484.1519, found: 484.1519. HPLC (Daicel Chiralpak OZ-H $(25 \mathrm{~cm})$, Hexanes/IPA = $50 / 50$ flow rate $=0.5 \mathrm{~mL} / \mathrm{min}, \mathrm{I}=280 \mathrm{~nm}$ ) $\mathrm{t}_{\mathrm{R}}=17.55 \mathrm{~min}$ (major), $31.08 \mathrm{~min}$ (minor).

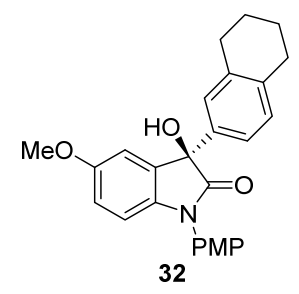




\section{(R)-3-Hydroxy-5-methoxy-1-(4-methoxyphenyl)-3-(5,6,7,8-tetrahydronaphthalen}

-2-yl)indolin-2-one (32): Prepared according to general procedures. Colorless oil (22.8 mg, 55\% yield, 92\% ee). Flash column chromatography conditions: petroleum ether/ethyl acetate $=2.5 / 1 .[\alpha]_{\mathrm{D}}{ }^{20}=+40.3\left(c 1.0, \mathrm{CHCl}_{3}\right) ;{ }^{1} \mathrm{H} \mathrm{NMR}\left(600 \mathrm{MHz}, \mathrm{CDCl}_{3}\right)$ $\delta=7.35(\mathrm{~d}, J=8.8,2 \mathrm{H}), 7.21(\mathrm{~s}, 1 \mathrm{H}), 7.14(\mathrm{~d}, J=8.0,1 \mathrm{H}), 7.03(\mathrm{t}, J=8.2,3 \mathrm{H}), 6.95$ $(\mathrm{d}, J=2.4,1 \mathrm{H}), 6.78(\mathrm{dd}, J=8.6,2.4,1 \mathrm{H}), 6.75(\mathrm{~d}, J=8.6,1 \mathrm{H}), 3.85(\mathrm{~s}, 3 \mathrm{H}), 3.75(\mathrm{~s}$, $3 \mathrm{H}), 3.68(\mathrm{~s}, 1 \mathrm{H}), 2.74(\mathrm{~s}, 4 \mathrm{H}), 1.88-1.72(\mathrm{~m}, 4 \mathrm{H}) .{ }^{13} \mathrm{C} \mathrm{NMR}\left(150 \mathrm{MHz}, \mathrm{CDCl}_{3}\right) \delta$ 177.2, 159.2, 156.7, 137.6, 137.5, 137.4, 137.2, 132.6, 129.5, 127.7, 125.8, 122.4, 114.9, 114.7, 111.5, 110.5, 78.3, 55.8, 55.6, 29.6, 29.2, 23.1. HR-ESI-MS calcd. for $\mathrm{C}_{26} \mathrm{H}_{26} \mathrm{NO}_{4}[\mathrm{M}+\mathrm{H}]^{+}:$416.1856, found: 416.1858. HPLC (Daicel Chiralpak OZ-H (25 $\mathrm{cm})$, Hexanes $/ \mathrm{IPA}=60 / 40$ flow rate $=0.5 \mathrm{~mL} / \mathrm{min}, \mathrm{I}=280 \mathrm{~nm}) \mathrm{t}_{\mathrm{R}}=19.65 \mathrm{~min}$ (major), $22.85 \mathrm{~min}$ (minor).

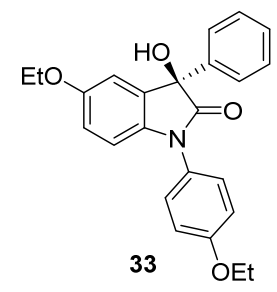

(R)-5-Ethoxy-1-(4-ethoxyphenyl)-3-hydroxy-3-phenylindolin-2-one (33): Prepared according to general procedures. Light yellow oil (38.5 mg, 99\% yield, 93\% ee). Flash column chromatography conditions: petroleum ether/ethyl acetate $=2.5 / 1 .[\alpha] \mathrm{D}^{20}=$ $+56.3\left(c\right.$ 1.0, $\left.\mathrm{CHCl}_{3}\right) ;{ }^{1} \mathrm{H} \mathrm{NMR}\left(600 \mathrm{MHz}, \mathrm{CDCl}_{3}\right) \delta=7.47(\mathrm{~d}, J=7.2,2 \mathrm{H}), 7.40-7.28$ $(\mathrm{m}, 5 \mathrm{H}), 7.01(\mathrm{~d}, J=8.9,2 \mathrm{H}), 6.92(\mathrm{~d}, J=2.5,1 \mathrm{H}), 6.78(\mathrm{dd}, J=8.6,2.5,1 \mathrm{H}), 6.74(\mathrm{~d}$, $J=8.6,1 \mathrm{H}), 4.08(\mathrm{q}, J=7.0,2 \mathrm{H}), 3.95(\mathrm{q}, J=7.0,2 \mathrm{H}), 3.75(\mathrm{~s}, 1 \mathrm{H}), 1.44(\mathrm{t}, J=7.0$, $3 \mathrm{H}), 1.36(\mathrm{t}, J=7.0,3 \mathrm{H}) .{ }^{13} \mathrm{C} \mathrm{NMR}\left(150 \mathrm{MHz}, \mathrm{CDCl}_{3}\right) \delta 177.0,158.6,156.1,140.6$, 137.1, 132.5, 128.7, 128.3, 127.6, 126.8, 125.3, 115.48, 115.45, 112.3, 110.6, 78.4, 64.2, 63.8, 14.80, 14.78. HR-ESI-MS calcd. for $\mathrm{C}_{24} \mathrm{H}_{23} \mathrm{NO}_{4} \mathrm{Na}[\mathrm{M}+\mathrm{Na}]^{+}: 412.1519$, found: 412.1520. HPLC (Daicel Chiralpak OZ-H $(25 \mathrm{~cm})$, Hexanes/IPA $=50 / 50$ flow rate $=$ $0.5 \mathrm{~mL} / \mathrm{min}, \mathrm{I}=280 \mathrm{~nm}$ ) $\mathrm{t}_{\mathrm{R}}=22.41 \mathrm{~min}$ (major), $15.83 \mathrm{~min}$ (minor). 


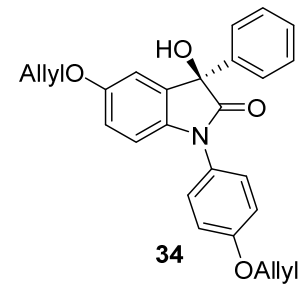

(R)-5-(Allyloxy)-1-(4-(allyloxy)phenyl)-3-hydroxy-3-phenylindolin-2-one

(34):

Prepared according to general procedures. Colorless oil (40.9 mg, 99\% yield, 93\% ee). Flash column chromatography conditions: petroleum ether/ethyl acetate $=2.5 / 1 .[\alpha]_{\mathrm{D}}^{20}$ $=+50.4\left(c 1.0, \mathrm{CHCl}_{3}\right) ;{ }^{1} \mathrm{H} \mathrm{NMR}\left(600 \mathrm{MHz}, \mathrm{CDCl}_{3}\right) \delta=7.46(\mathrm{~d}, J=7.3,2 \mathrm{H}), 7.39-$ $7.28(\mathrm{~m}, 5 \mathrm{H}), 7.03(\mathrm{~d}, J=8.8,2 \mathrm{H}), 6.94(\mathrm{~d}, J=2.5,1 \mathrm{H}), 6.81(\mathrm{dd}, J=8.6,2.5,1 \mathrm{H})$, $6.75(\mathrm{~d}, J=8.6,1 \mathrm{H}), 6.07(\mathrm{ddd}, J=22.4,10.5,5.3,1 \mathrm{H}), 5.99(\mathrm{ddd}, J=22.4,10.5,5.3$, $1 \mathrm{H}), 5.45(\mathrm{~d}, J=17.2,1 \mathrm{H}), 5.40-5.30(\mathrm{~m}, 2 \mathrm{H}), 5.25(\mathrm{~d}, J=10.5,1 \mathrm{H}), 4.58(\mathrm{~d}, J=5.2$, $2 \mathrm{H}), 4.45(\mathrm{~d}, J=5.3,2 \mathrm{H}), 3.96(\mathrm{~s}, 1 \mathrm{H}) .{ }^{13} \mathrm{C} \mathrm{NMR}\left(150 \mathrm{MHz}, \mathrm{CDCl}_{3}\right) \delta$ 177.0, 158.2, 155.8, 140.5, 137.3, 133.1, 133.0, 132.6, 128.7, 128.3, 127.6, 127.0, 125.3, 118.0, 117.8, 115.8, 115.7, 112.6, 110.5, 78.4, 69.5, 69.1. HR-ESI-MS calcd. for $\mathrm{C}_{26} \mathrm{H}_{23} \mathrm{NO}_{4} \mathrm{Na}[\mathrm{M}+$ $\mathrm{Na}]^{+}:$436.1519, found: 436.1520. HPLC (Daicel Chiralpak OZ-H $(25 \mathrm{~cm})$, Hexanes $/ \mathrm{IPA}=50 / 50$ flow rate $=0.5 \mathrm{~mL} / \mathrm{min}, \mathrm{I}=280 \mathrm{~nm}$ ) $\mathrm{t}_{\mathrm{R}}=24.35 \mathrm{~min}$ (major), $15.98 \min$ (minor).

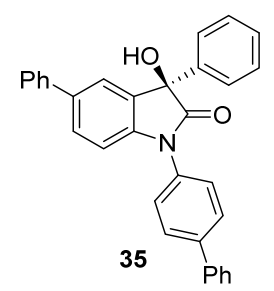

(R)-1-([1,1'-Biphenyl]-4-yl)-3-hydroxy-3,5-diphenylindolin-2-one (35): Prepared according to general procedures. Brown oil $(23.1 \mathrm{mg}, 51 \%$ yield, $87 \%$ ee). Flash column chromatography conditions: petroleum ether/ethyl acetate $=5 / 1 .[\alpha]_{\mathrm{D}}{ }^{20}=+10.1$ $\left(c\right.$ 1.0, $\left.\mathrm{CHCl}_{3}\right) ;{ }^{1} \mathrm{H} \mathrm{NMR}\left(600 \mathrm{MHz}, \mathrm{CDCl}_{3}\right) \delta=7.77(\mathrm{~d}, J=8.3,2 \mathrm{H}), 7.66(\mathrm{~d}, J=8.3$, 3H), $7.61-7.54(\mathrm{~m}, 7 \mathrm{H}), 7.52(\mathrm{t}, J=7.6,2 \mathrm{H}), 7.45-7.39(\mathrm{~m}, 5 \mathrm{H}), 7.37-7.34(\mathrm{~m}$, 2H), $7.07(\mathrm{~d}, J=8.2,1 \mathrm{H}), 4.35(\mathrm{~s}, 1 \mathrm{H}) .{ }^{13} \mathrm{C} \mathrm{NMR}\left(150 \mathrm{MHz}, \mathrm{CDCl}_{3}\right) \delta 177.3,142.7$, $141.3,140.42,140.37,140.2$, 137.5, 133.2, 132.3, 129.0, 128.9, 128.8, 128.51, 128.46, 127.8, 127.33, 127.28, 126.9, 126.7, 125.5, 124.3, 110.5, 78.4. HR-ESI-MS calcd. for 
$\mathrm{C}_{32} \mathrm{H}_{23} \mathrm{NO}_{2} \mathrm{Na}[\mathrm{M}+\mathrm{Na}]^{+}:$476.1621, found: 476.1625. HPLC (Daicel Chiralpak OZ-H $(25 \mathrm{~cm})$, Hexanes $/ \mathrm{IPA}=50 / 50$ flow rate $=0.5 \mathrm{~mL} / \mathrm{min}, \mathrm{I}=280 \mathrm{~nm}) \mathrm{t}_{\mathrm{R}}=25.10 \mathrm{~min}$ (major), $18.68 \min$ (minor).

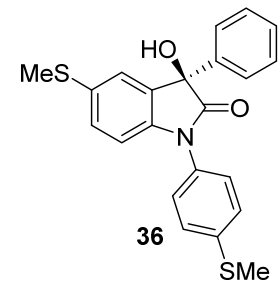

\section{(R)-3-Hydroxy-5-(methylthio)-1-(4-(methylthio)phenyl)-3-phenylindolin-2-one}

(36): Prepared according to general procedures. Colorless oil (21.3 mg, 54\% yield, 91\% ee). Flash column chromatography conditions: petroleum ether/ethyl acetate $=5 / 1$. $[\alpha]_{\mathrm{D}^{20}}=+0.9\left(c 1.0, \mathrm{CHCl}_{3}\right) ;{ }^{1} \mathrm{H} \mathrm{NMR}\left(600 \mathrm{MHz}, \mathrm{CDCl}_{3}\right) \delta=7.44(\mathrm{~d}, J=7.0,2 \mathrm{H})$, $7.40-7.29(\mathrm{~m}, 7 \mathrm{H}), 7.27(\mathrm{~d}, J=1.8,1 \mathrm{H}), 7.20(\mathrm{dd}, J=8.3,1.8,1 \mathrm{H}), 6.80(\mathrm{~d}, J=8.3$, 1H), $4.29(\mathrm{~s}, 1 \mathrm{H}), 2.51(\mathrm{~s}, 3 \mathrm{H}), 2.41(\mathrm{~s}, 3 \mathrm{H}) .{ }^{13} \mathrm{C} \mathrm{NMR}\left(150 \mathrm{MHz}, \mathrm{CDCl}_{3}\right) \delta 176.78$, $176.76,141.3,140.1,139.2,133.6,132.5,132.4,130.8,129.29,129.26,128.8,128.5$, 127.4, 126.7, 125.4, 125.3, 125.1, 110.4, 78.1, 17.3, 15.8. HR-ESI-MS calcd. for $\mathrm{C}_{22} \mathrm{H}_{19} \mathrm{NO}_{2} \mathrm{~S}_{2} \mathrm{Na}[\mathrm{M}+\mathrm{Na}]^{+}:$416.0749, found: 416.0748. HPLC (Daicel Chiralpak OZ$\mathrm{H}(25 \mathrm{~cm})$, Hexanes $/ \mathrm{IPA}=65 / 35$ flow rate $=0.5 \mathrm{~mL} / \mathrm{min}, \mathrm{I}=280 \mathrm{~nm}) \mathrm{t}_{\mathrm{R}}=31.91 \mathrm{~min}$ (major), $36.66 \min$ (minor).

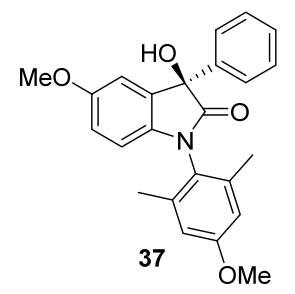

(R)-3-Hydroxy-5-methoxy-1-(4-methoxy-2,6-dimethylphenyl)-3-phenylindolin-2one (37): Prepared according to general procedures. Colorless oil (22.9 mg, 59\% yield, 93\% ee). Flash column chromatography conditions: petroleum ether/ethyl acetate $=4 / 1$. $[\alpha]_{\mathrm{D}}^{20}=+24.4\left(c 1.0, \mathrm{CHCl}_{3}\right) ;{ }^{1} \mathrm{H} \mathrm{NMR}\left(600 \mathrm{MHz}, \mathrm{CDCl}_{3}\right) \delta=7.50-7.44(\mathrm{~m}, 2 \mathrm{H})$, $7.36(\mathrm{t}, J=7.4,2 \mathrm{H}), 7.34-7.28(\mathrm{~m}, 1 \mathrm{H}), 7.08(\mathrm{~s}, 2 \mathrm{H}), 6.92(\mathrm{~d}, J=2.2,1 \mathrm{H}), 6.82-6.74$ $(\mathrm{m}, 2 \mathrm{H}), 3.76(\mathrm{~s}, 3 \mathrm{H}), 3.74(\mathrm{~s}, 3 \mathrm{H}), 2.33(\mathrm{~s}, 6 \mathrm{H}) .{ }^{13} \mathrm{C} \mathrm{NMR}\left(150 \mathrm{MHz}, \mathrm{CDCl}_{3}\right) \delta=176.9$, 
$156.8,156.7,140.5,137.2,132.5,129.5,128.7,128.3,126.6,125.3,114.8,111.5,110.8$, 78.4, 59.7, 55.8, 16.2. HR-ESI-MS calcd. for $\mathrm{C}_{24} \mathrm{H}_{23} \mathrm{NO}_{4} \mathrm{Na}[\mathrm{M}+\mathrm{Na}]^{+}$: 412.1519, found: 412.1521. HPLC (Daicel Chiralpak OZ-H $(25 \mathrm{~cm})$, Hexanes/IPA = 50/50 flow rate $=0.5 \mathrm{~mL} / \mathrm{min}, \mathrm{I}=280 \mathrm{~nm}$ ) $\mathrm{t}_{\mathrm{R}}=12.02 \mathrm{~min}$ (major), $10.62 \mathrm{~min}$ (minor).

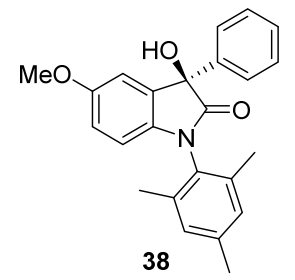

(R)-3-Hydroxy-1-mesityl-5-methoxy-3-phenylindolin-2-one (38): $\quad$ Prepared according to general procedures. Colorless oil $(23.9 \mathrm{mg}, 64 \%$ yield, $60 \%$ ee). Flash column chromatography conditions: petroleum ether/ethyl acetate $=2.5 / 1 .[\alpha]_{\mathrm{D}}^{20}=$ 40.3 (c 1.0, $\left.\mathrm{CHCl}_{3}\right) ;{ }^{1} \mathrm{H} \mathrm{NMR}\left(600 \mathrm{MHz}, \mathrm{CDCl}_{3}\right) \delta=7.49$ (d, $\left.J=7.3,2 \mathrm{H}\right), 7.37$ (t, $J=$ $7.4,2 \mathrm{H}), 7.33(\mathrm{t}, J=7.2,1 \mathrm{H}), 7.00(\mathrm{~d}, J=7.5,2 \mathrm{H}), 6.94(\mathrm{~d}, J=2.4,1 \mathrm{H}), 6.76(\mathrm{dd}, J=$ 8.5, 2.5, 1H), $6.36(\mathrm{~d}, J=8.5,1 \mathrm{H}), 3.74(\mathrm{~s}, 3 \mathrm{H}), 3.61(\mathrm{~s}, 1 \mathrm{H}), 2.34(\mathrm{~s}, 3 \mathrm{H}), 2.13(\mathrm{~s}, 3 \mathrm{H})$, $2.11(\mathrm{~s}, 3 \mathrm{H}) .{ }^{13} \mathrm{C} \mathrm{NMR}\left(150 \mathrm{MHz}, \mathrm{CDCl}_{3}\right) \delta=176.4,156.8,140.3,139.0,136.6,136.5$, $132.5,129.7,129.4,129.0,128.7,128.3,125.5,115.1,111.9,110.2,78.5,55.8,21.1$, 17.9, 17.7. HR-ESI-MS calcd. for $\mathrm{C}_{24} \mathrm{H}_{23} \mathrm{NO}_{3} \mathrm{Na}[\mathrm{M}+\mathrm{Na}]^{+}: 396.1570$, found: 396.1568 . HPLC (Daicel Chiralpak OZ-H $(25 \mathrm{~cm})$, Hexanes $/ \mathrm{IPA}=70 / 30$ flow rate $=0.5 \mathrm{~mL} / \mathrm{min}$, $\mathrm{I}=280 \mathrm{~nm}) \mathrm{t}_{\mathrm{R}}=14.02 \mathrm{~min}$ (major), $15.80 \mathrm{~min}$ (minor). 


\section{References}

[1] T. Karasawa, R. Oriez, N. Kumagai, M. Shibasaki, J. Am. Chem. Soc. 2018, 140, 12290-12295.

[2] S. K. Alamsetti, G. Sekar, Chem. Commun. 2010, 46, 7235-7237.

[3] Y. Wang, Y. Liu, K. Li, G. Yang, W. Zhang, Adv.Synth. Catal. 2017, 359, 1933 1941.

[4] Z. Zhang, J. Su, Z. Zha, Z. Wang, Chem. Eur. J. 2013, 19, 17711-17714.

[5] K. A. Rykaczewski, C. S. Schindler, Org. Lett. 2020, 22, 6516-6519.

[6] D. K. Das, V. K. Kumar Pampana, K. C. Hwang, Chem. Sci. 2018, 9, 73187326.

[7] T. Gensch, M. Rönnefahrt, R. Czerwonka, A. Jäger, O. Kataeva, I. Bauer, H.-J. Knölker, Chem. Eur. J. 2012, 18, 770-776.

[8] L. L. Hill, J. L. Crowell, S. L. Tutwiler, N. L. Massie, C. C. Hines, S. T. Griffin, R. D. Rogers, K. H. Shaughnessy, G. A. Grasa, C. C. C. Johansson Seechurn, H. Li, T. J. Colacot, J. Chou, C. J. Woltermann, J. Org. Chem. 2010, $75,6477-6488$. 


\section{Copies of ${ }^{1} \mathrm{H},{ }^{13} \mathrm{C}$ and ${ }^{19} \mathrm{~F}$ NMR Spectra}

${ }^{1} \mathrm{H} \mathrm{NMR}\left(600 \mathrm{MHz}, \mathrm{CDCl}_{3}\right)$ of $\mathbf{3}$
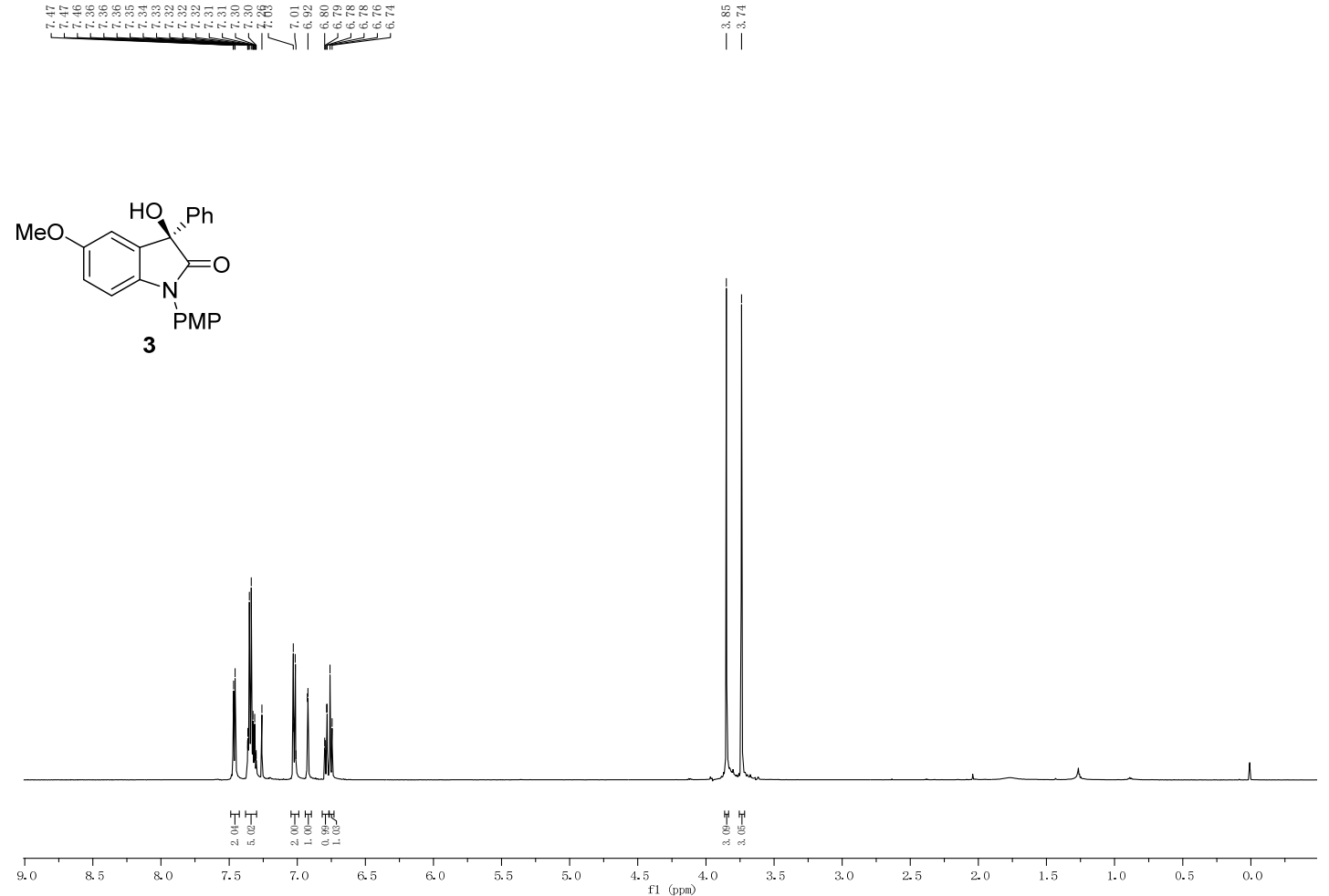

${ }^{13} \mathrm{C} \mathrm{NMR}\left(150 \mathrm{MHz}, \mathrm{CDCl}_{3}\right)$ of 3

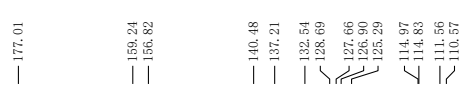
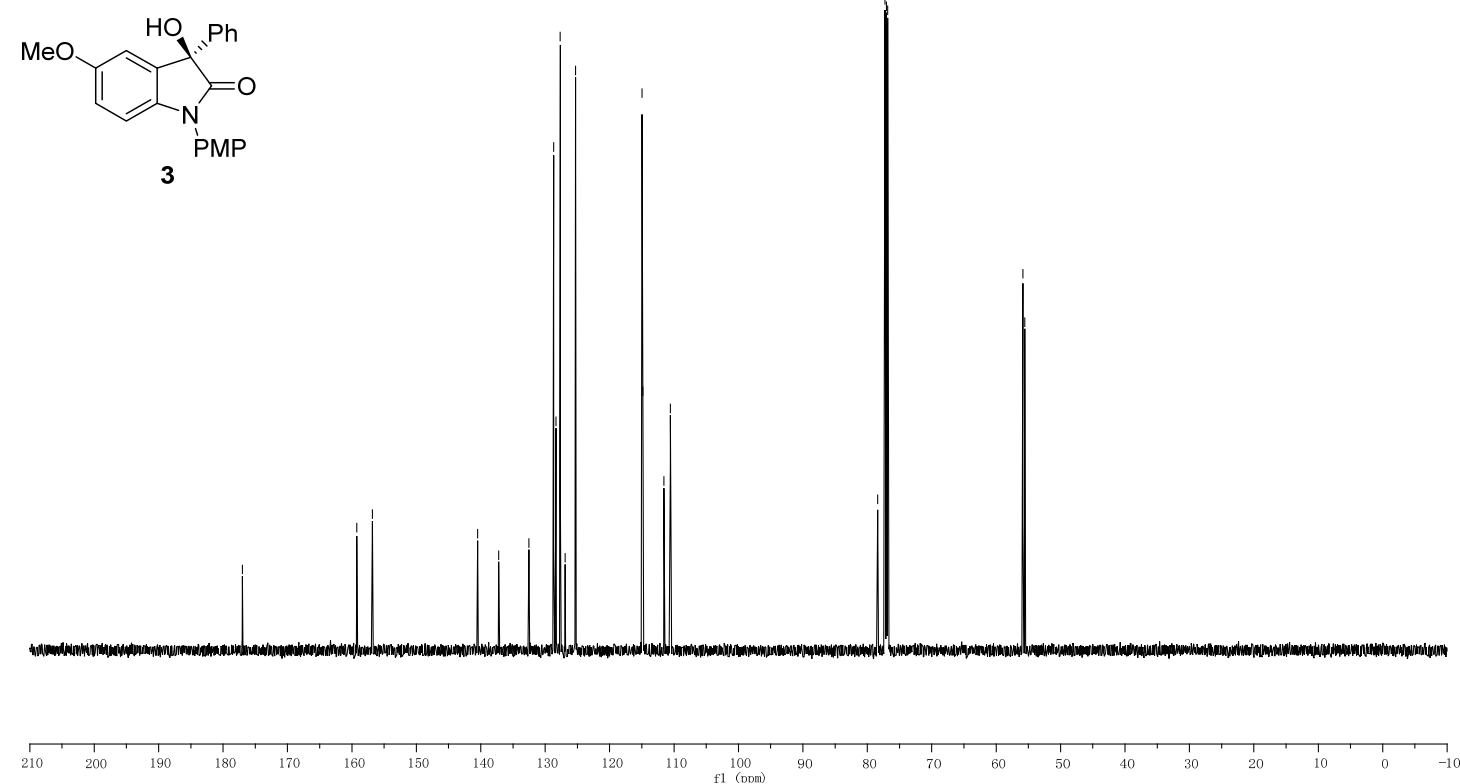
${ }^{1} \mathrm{H}$ NMR (600 MHz, $\left.\mathrm{CDCl}_{3}\right)$ of 4

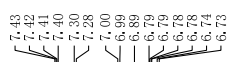


${ }^{13} \mathrm{C}$ NMR $\left(150 \mathrm{MHz}, \mathrm{CDCl}_{3}\right)$ of 4
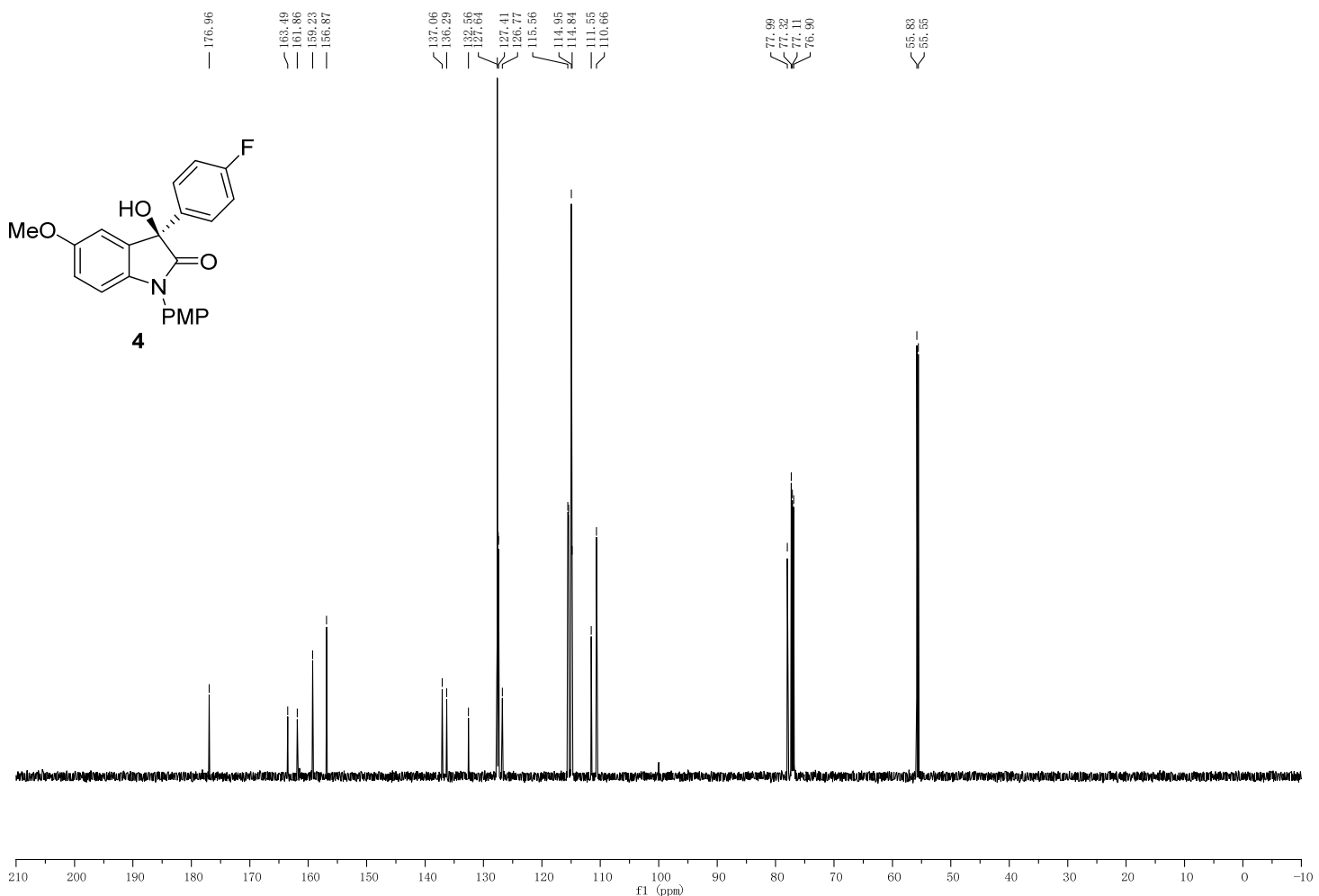
${ }^{19} \mathrm{~F}$ NMR $\left(565 \mathrm{MHz}, \mathrm{CDCl}_{3}\right)$ of 4
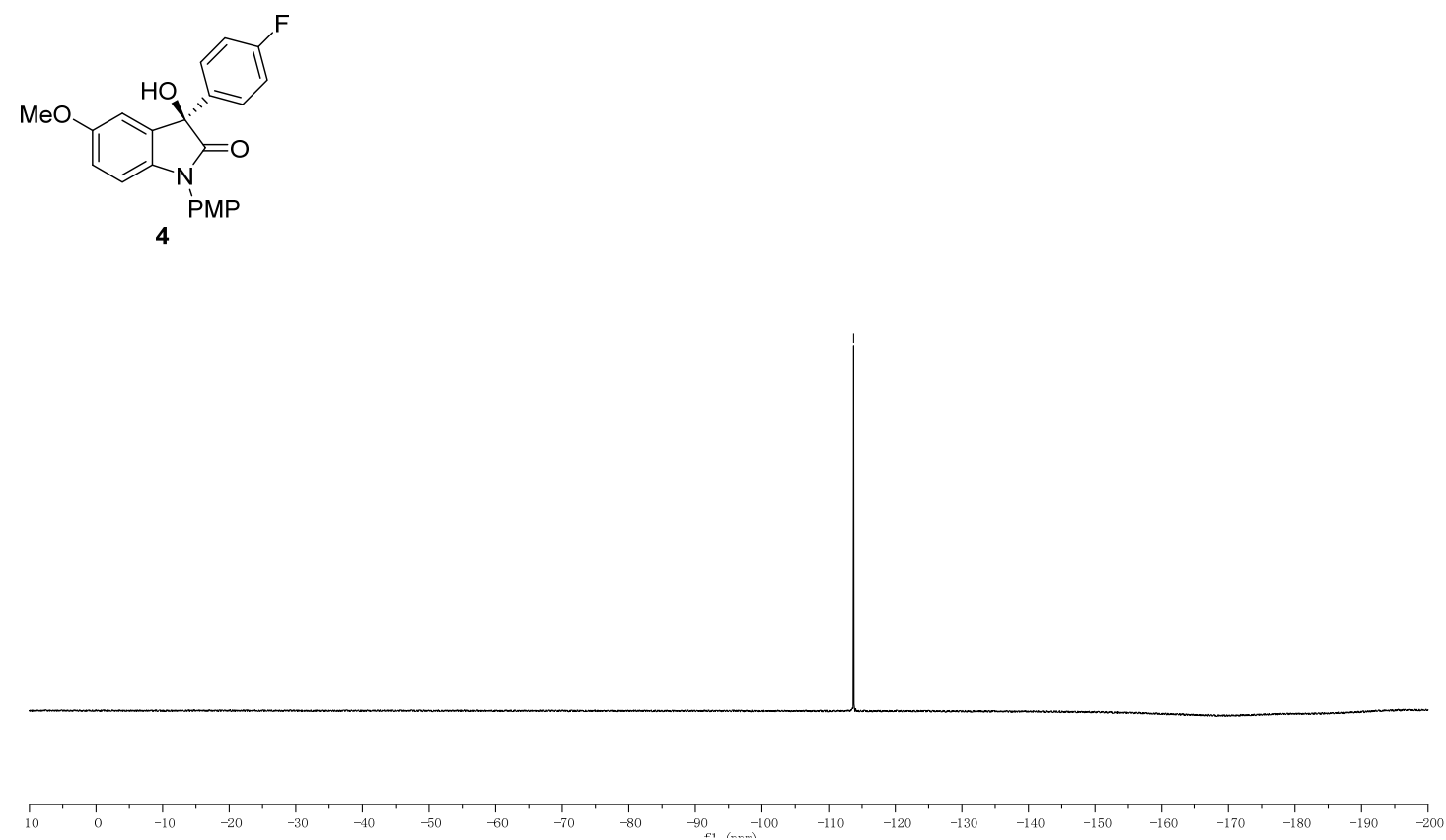
${ }^{1} \mathrm{H}$ NMR (600 MHz, $\left.\mathrm{CDCl}_{3}\right)$ of 5

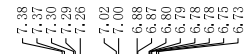<smiles>COc1ccc2c(c1)C(c1ccc(Cl)cc1)C(=O)N2[13C](=O)[O-]</smiles>
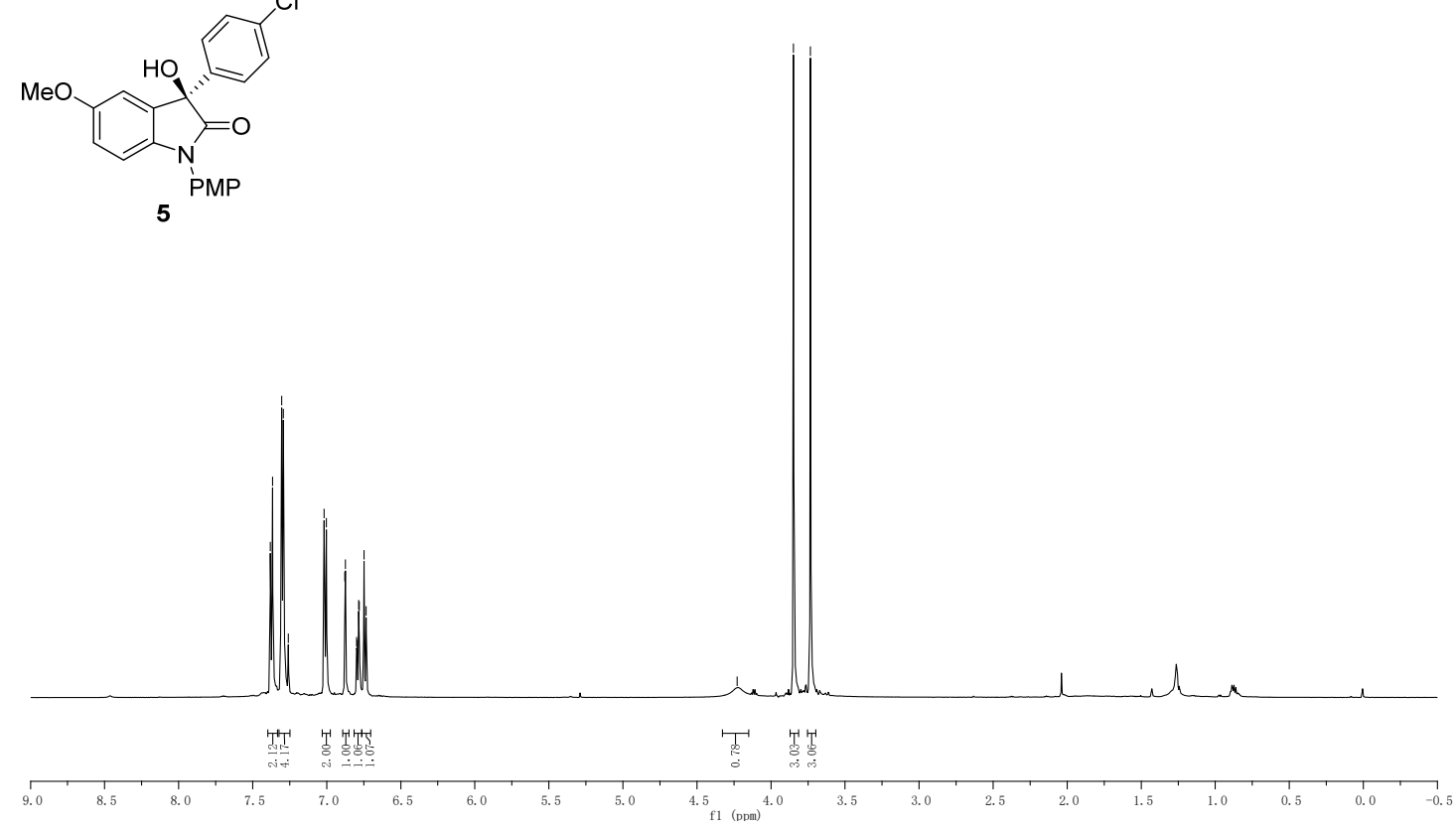

${ }^{13} \mathrm{C}$ NMR (150 MHz, $\left.\mathrm{CDCl}_{3}\right)$ of 5
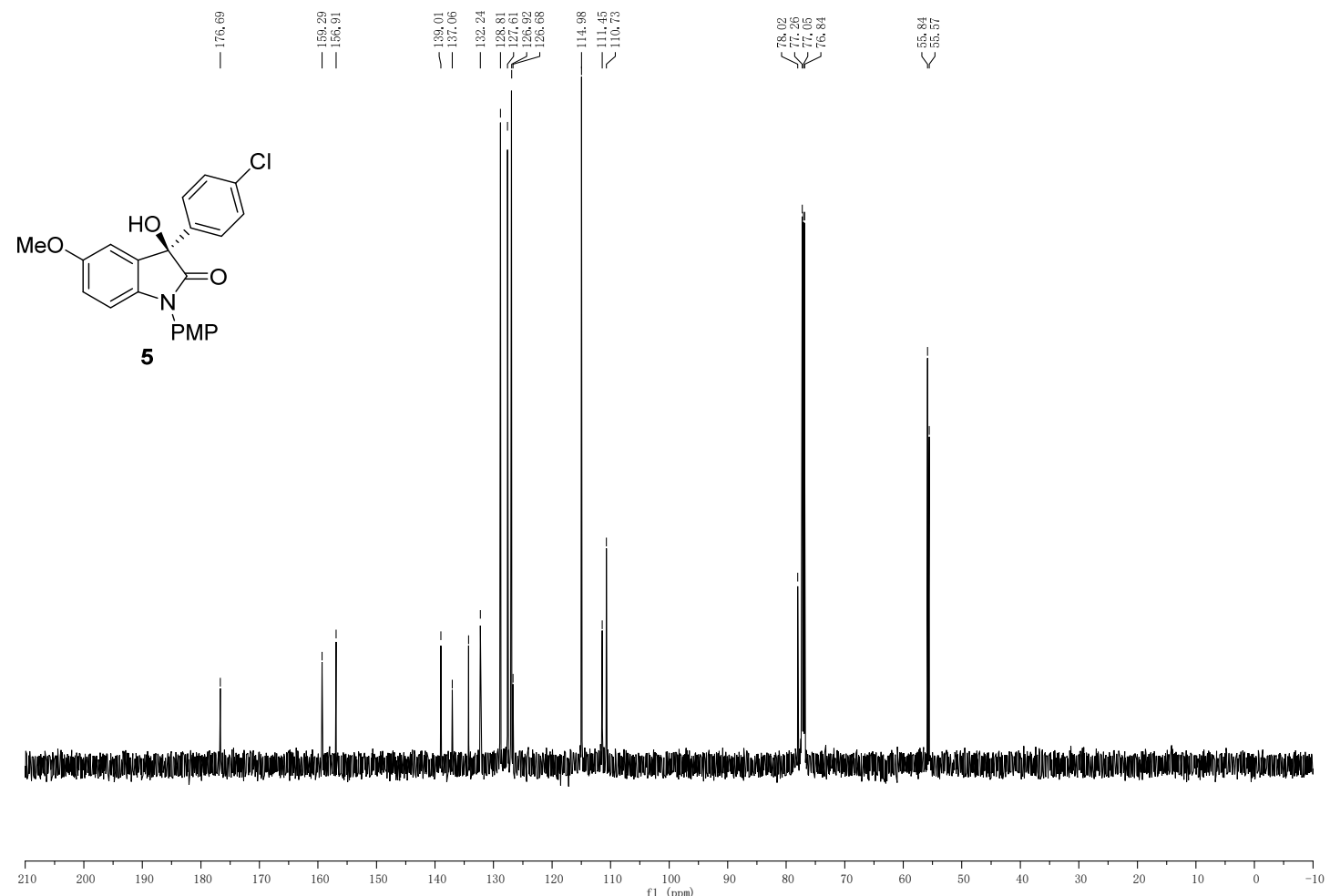
${ }^{1} \mathrm{H}$ NMR (600 MHz, $\left.\mathrm{CDCl}_{3}\right)$ of $\mathbf{6}$

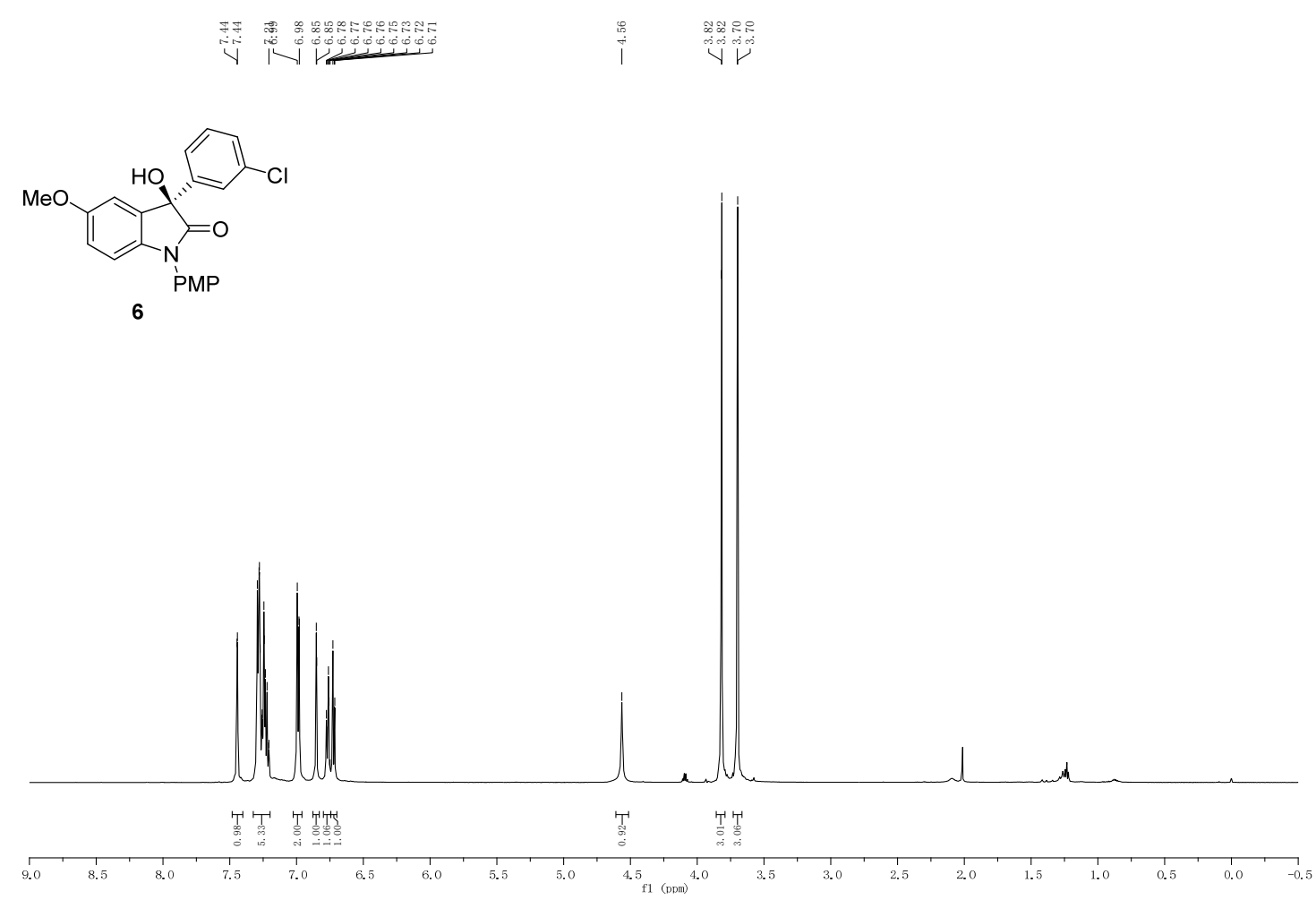

${ }^{13} \mathrm{C}$ NMR (150 MHz, $\left.\mathrm{CDCl}_{3}\right)$ of 6
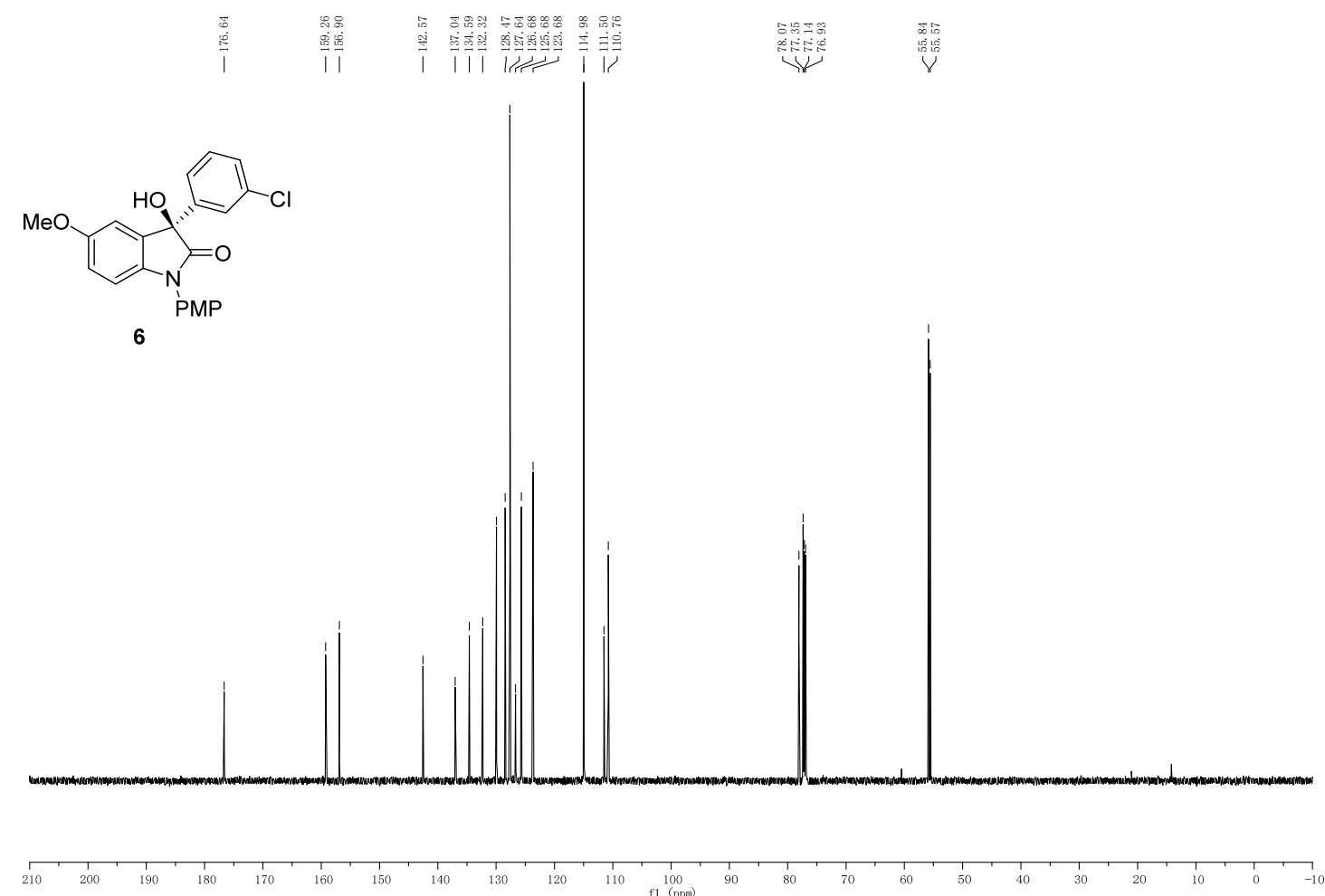
${ }^{1} \mathrm{H}$ NMR (600 MHz, MeOD) of 7

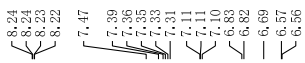
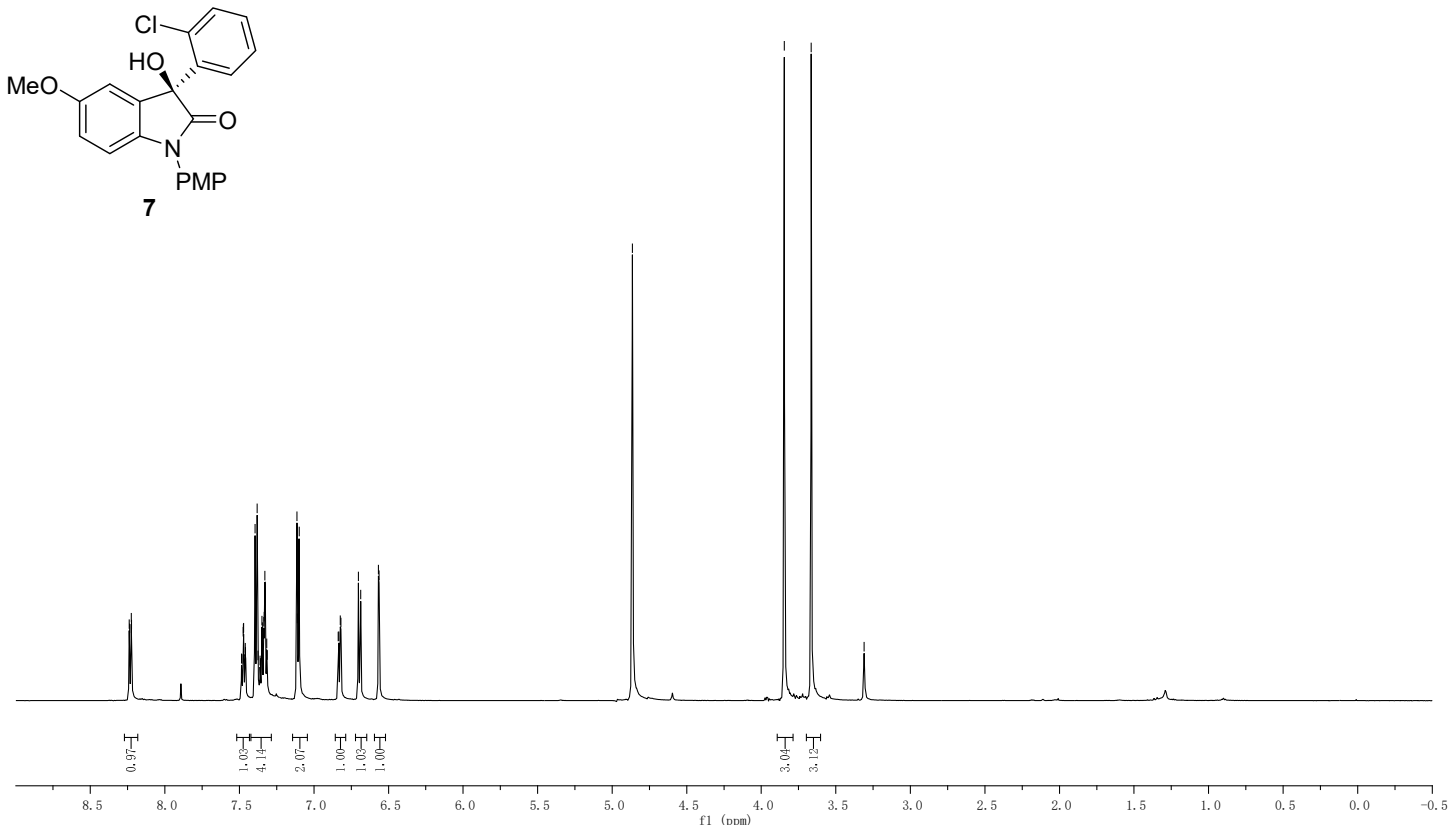

${ }^{13} \mathrm{C}$ NMR (150 MHz, MeOD) of 7

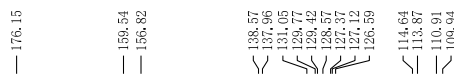

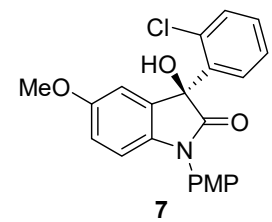

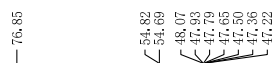

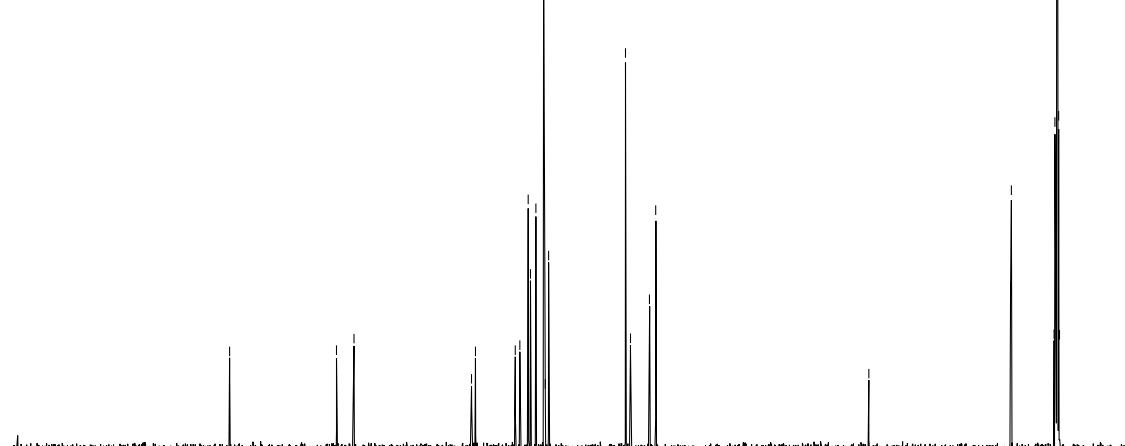

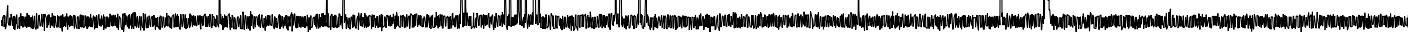

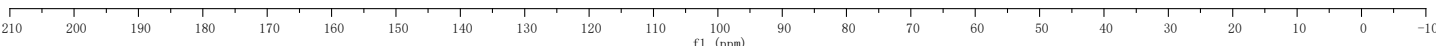


${ }^{1} \mathrm{H}$ NMR (600 MHz, $\left.\mathrm{CDCl}_{3}\right)$ of 8

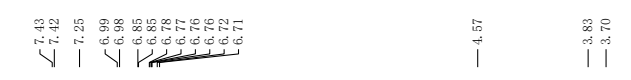

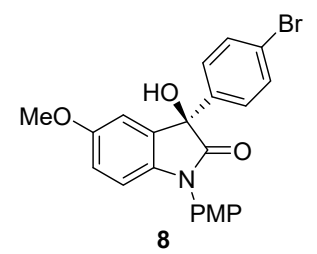

8
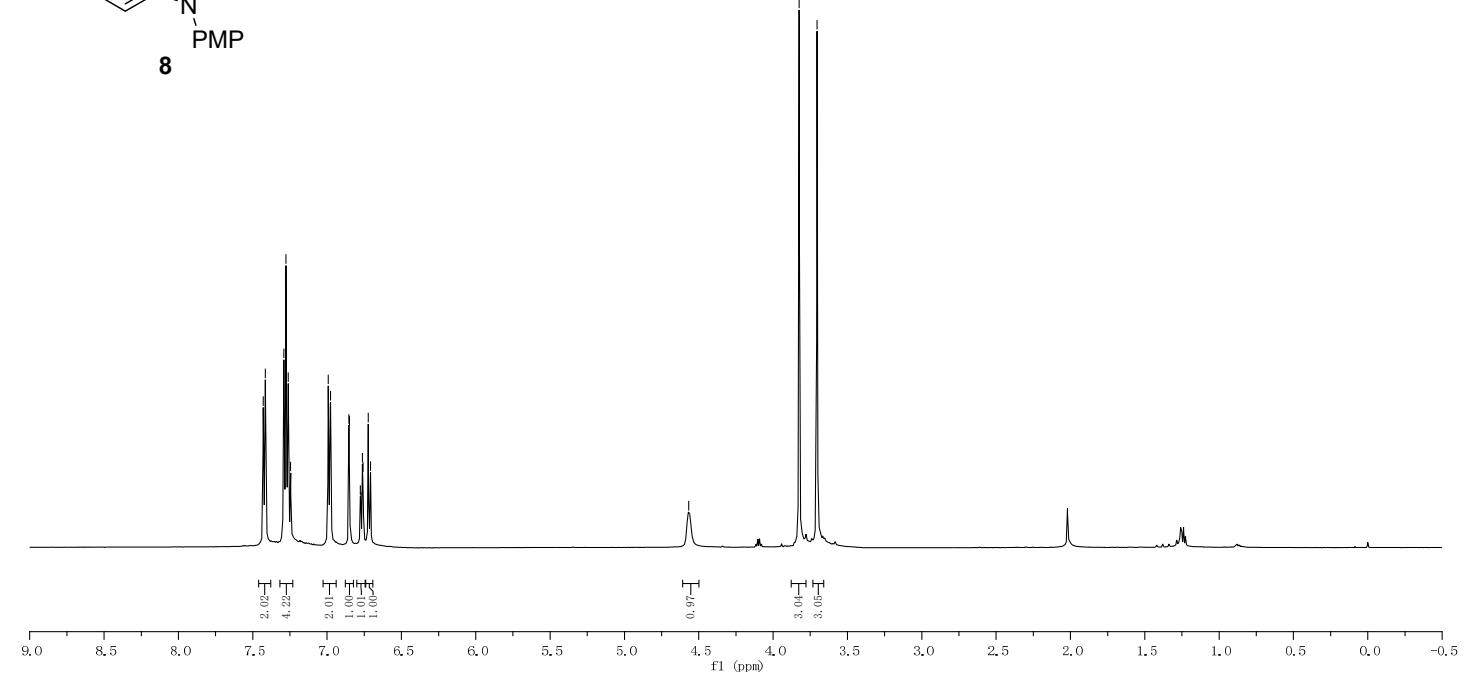

${ }^{13} \mathrm{C}$ NMR (150 MHz, $\left.\mathrm{CDCl}_{3}\right)$ of 8
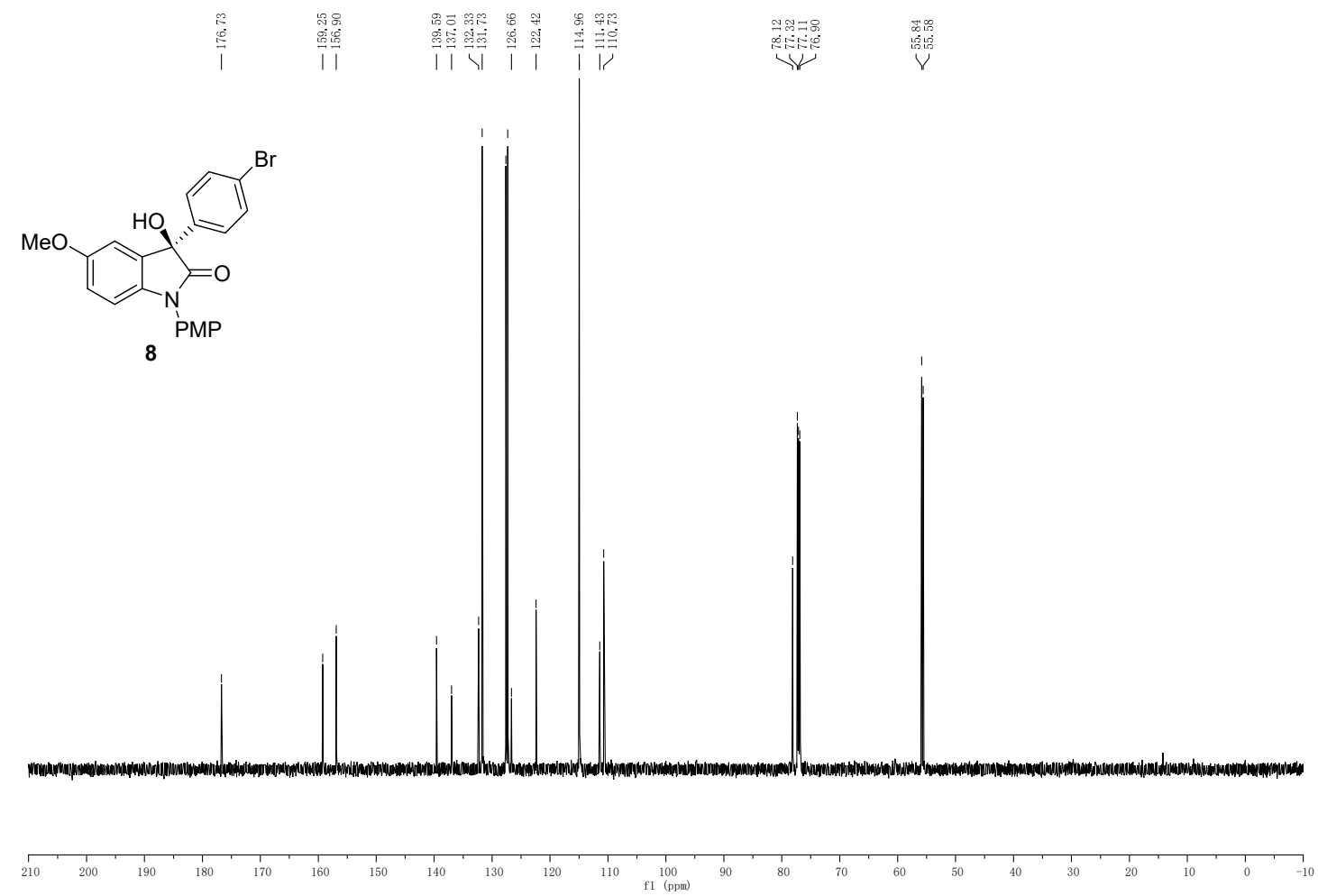
${ }^{1} \mathrm{H}$ NMR $\left(600 \mathrm{MHz}, \mathrm{CDCl}_{3}\right)$ of 9
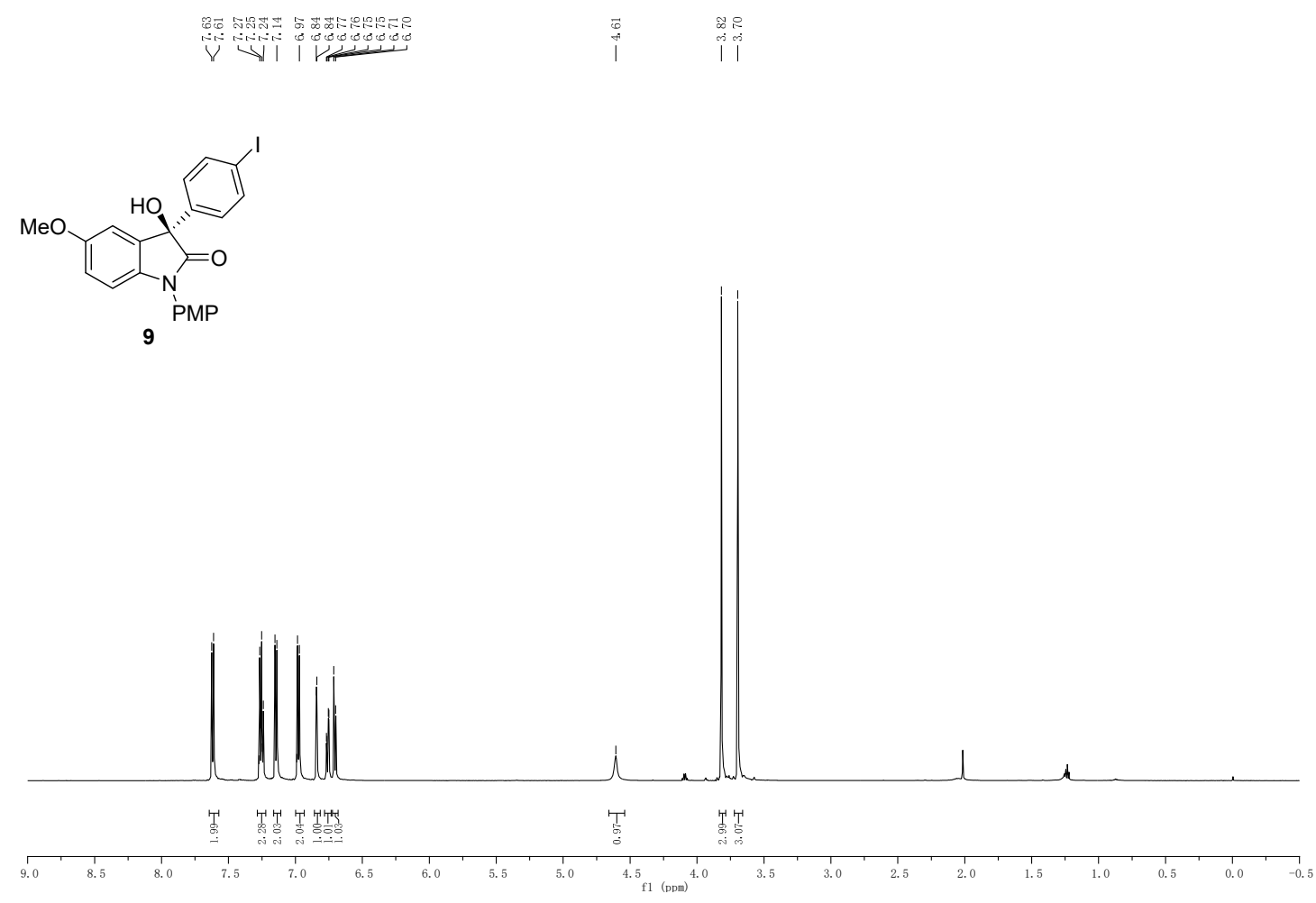

${ }^{13} \mathrm{C}$ NMR (150 MHz, $\left.\mathrm{CDCl}_{3}\right)$ of 9
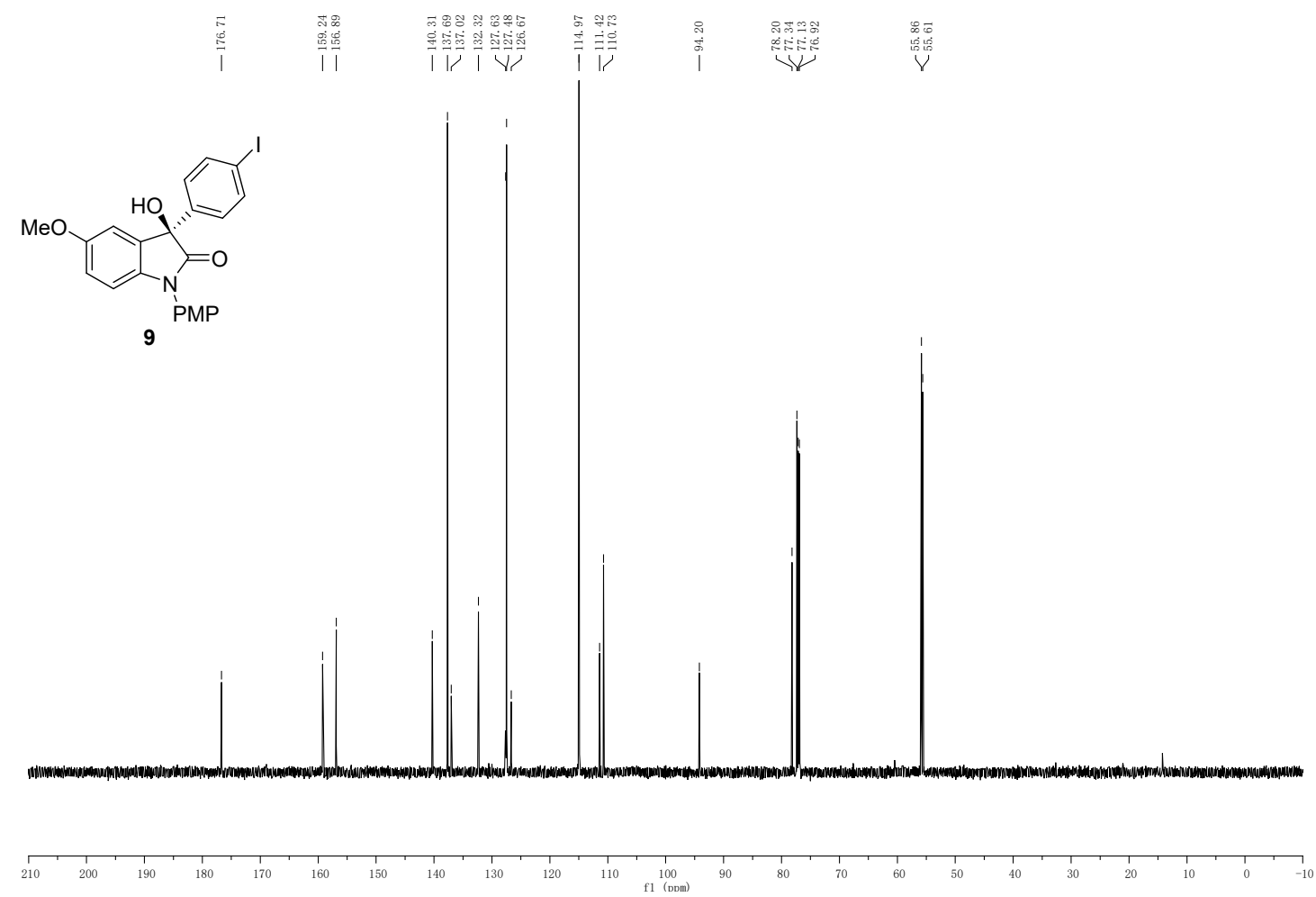
${ }^{1} \mathrm{H}$ NMR $\left(600 \mathrm{MHz}, \mathrm{CDCl}_{3}\right)$ of $\mathbf{1 0}$

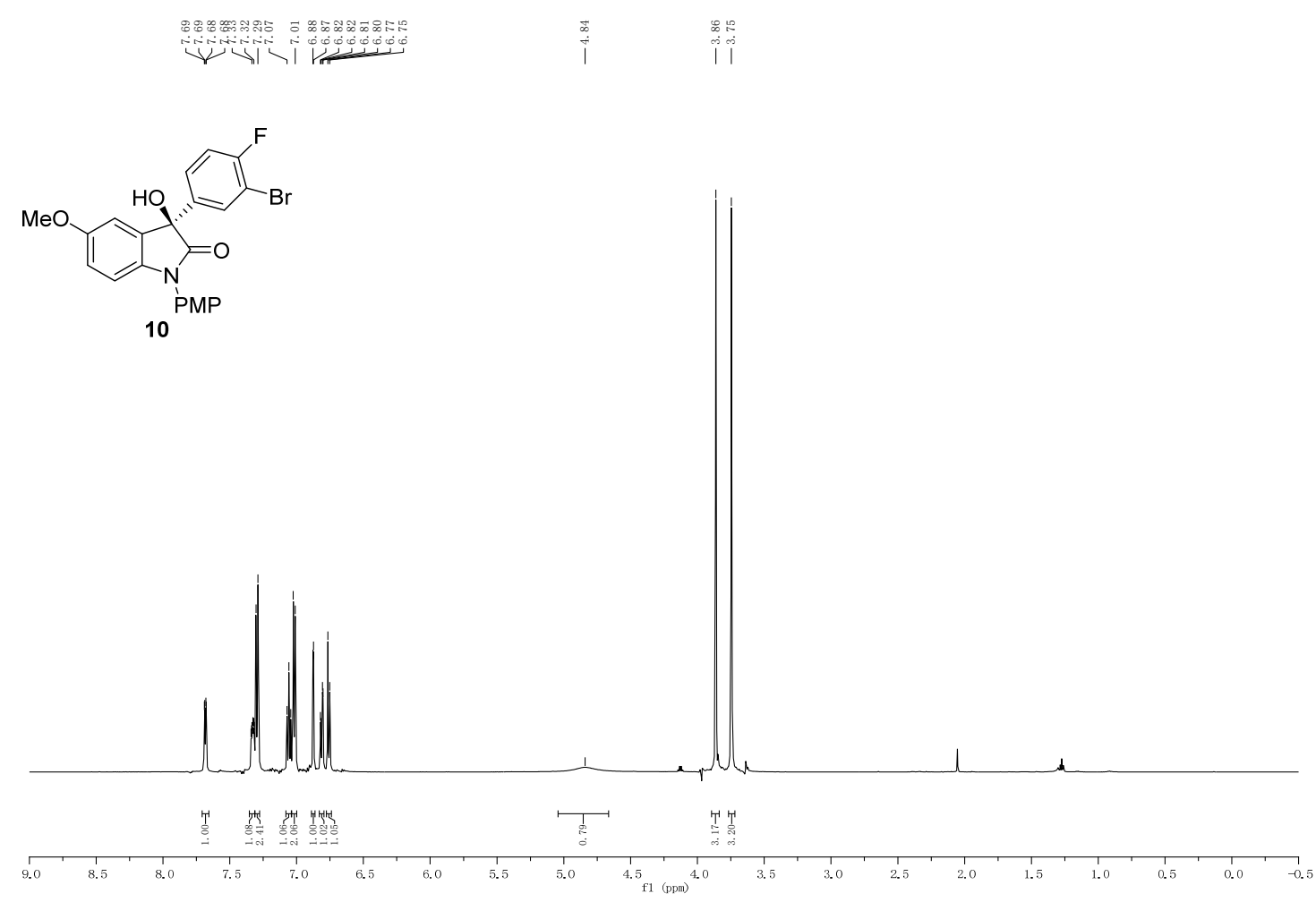

${ }^{13} \mathrm{C}$ NMR (150 MHz, $\left.\mathrm{CDCl}_{3}\right)$ of $\mathbf{1 0}$
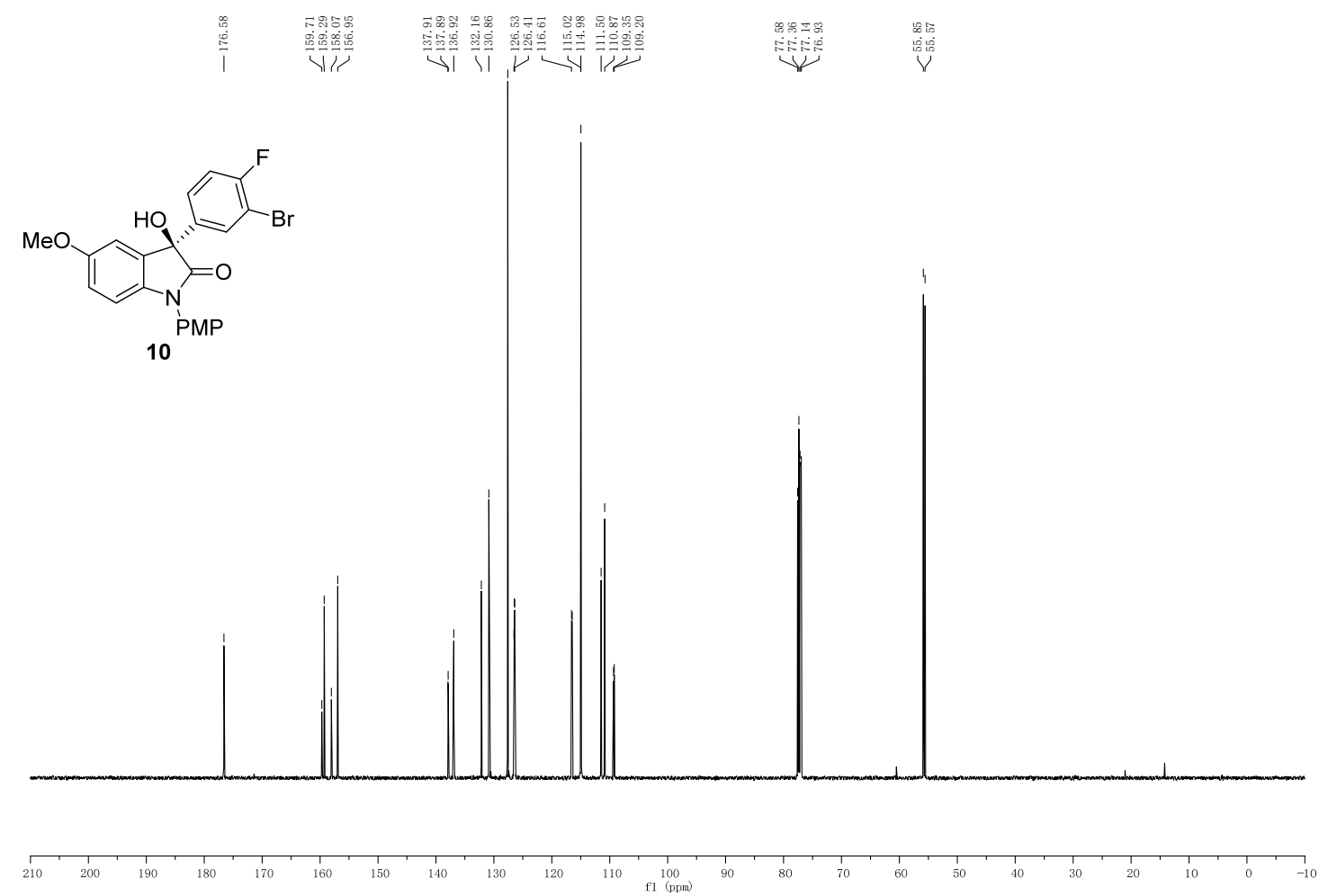
${ }^{19} \mathrm{~F}$ NMR (565 MHz, $\left.\mathrm{CDCl}_{3}\right)$ of $\mathbf{1 0}$
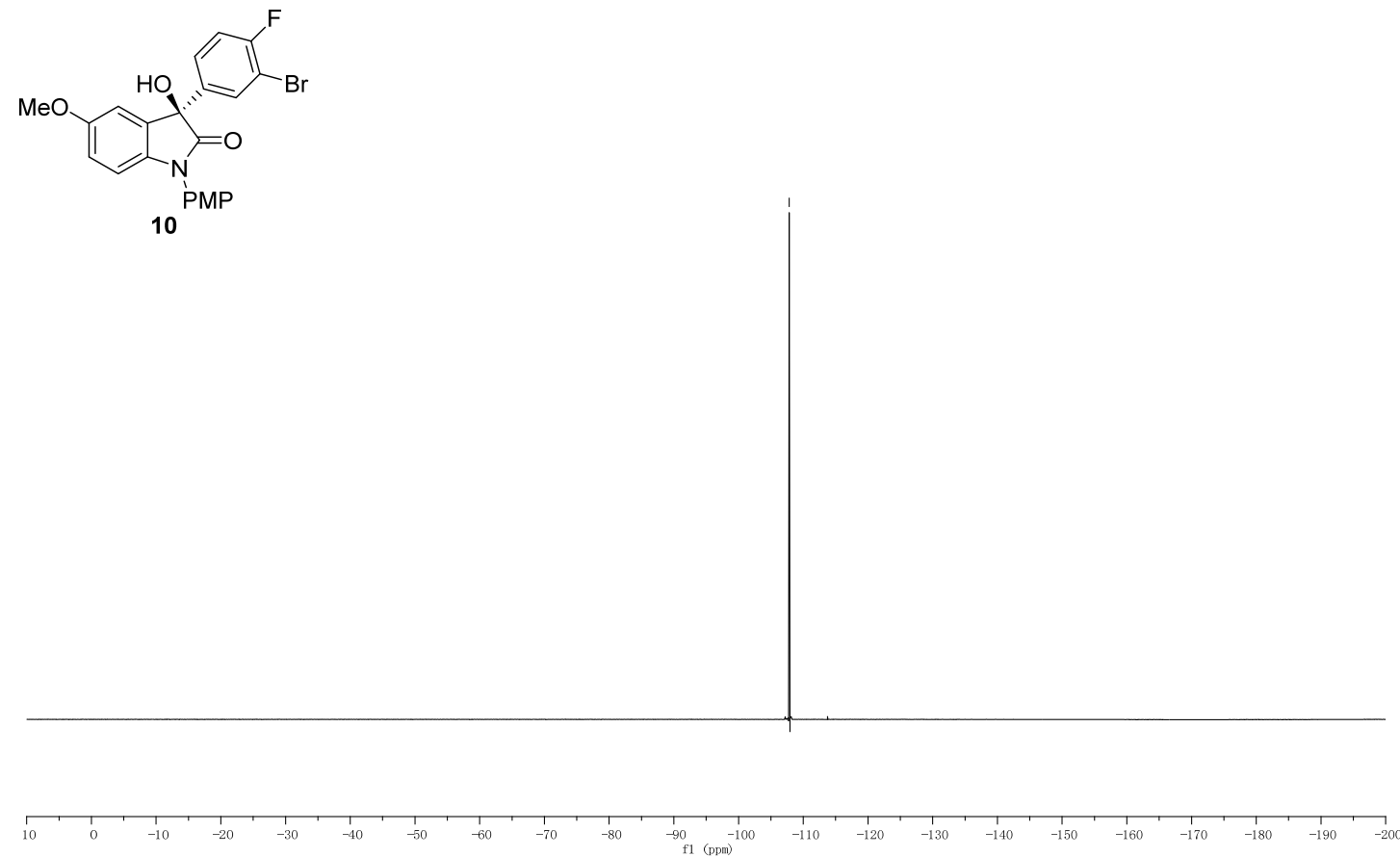
${ }^{1} \mathrm{H}$ NMR (600 MHz, $\left.\mathrm{CDCl}_{3}\right)$ of $\mathbf{1 1}$

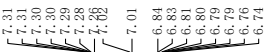
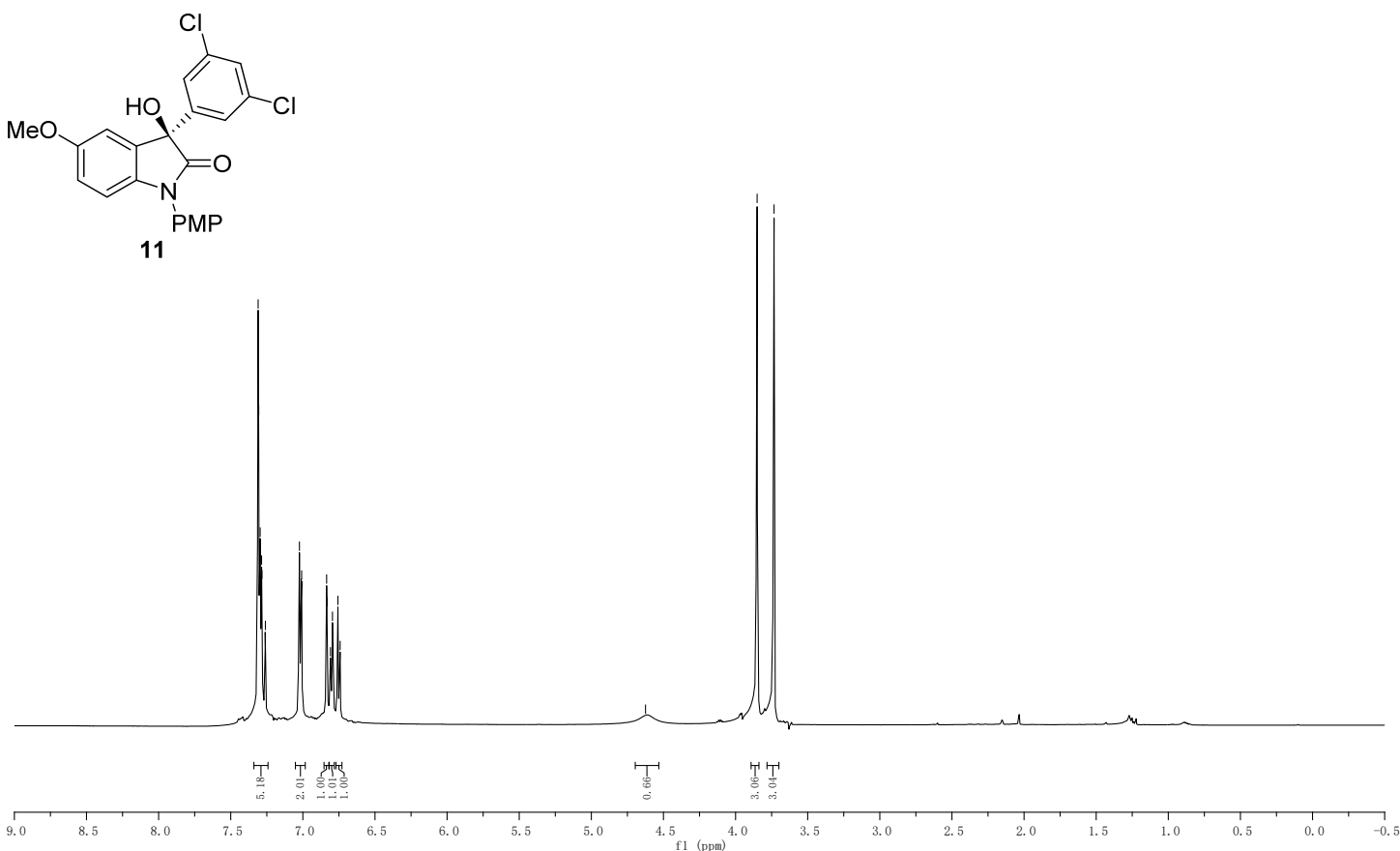

${ }^{13} \mathrm{C} \mathrm{NMR}\left(150 \mathrm{MHz}, \mathrm{CDCl}_{3}\right)$ of $\mathbf{1 1}$

${ }^{1} \mathrm{H}$ NMR (600 MHz, $\mathrm{CDCl}_{3}$ ) of $\mathbf{1 2}$

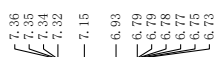

总部昂

(12)

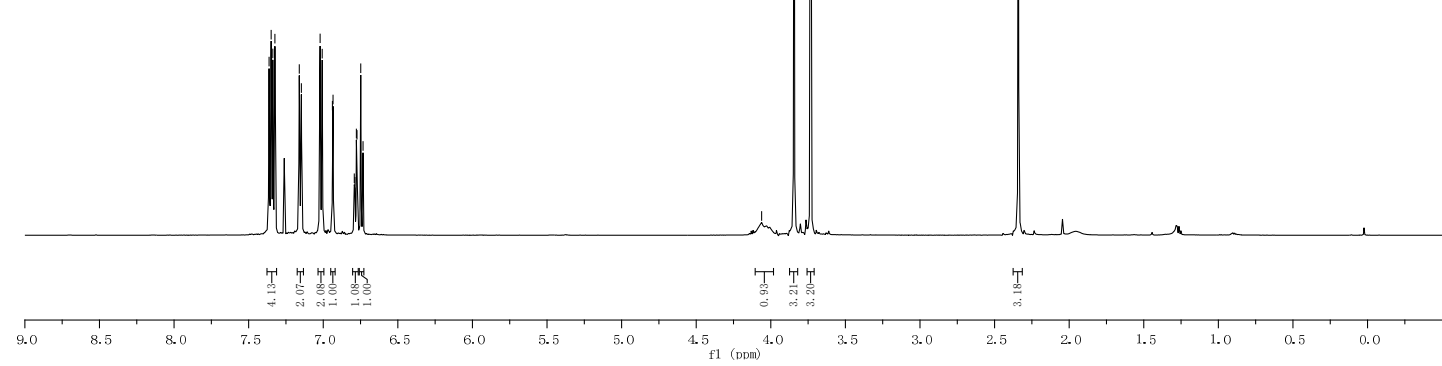

${ }^{13} \mathrm{C}$ NMR (150 MHz, $\mathrm{CDCl}_{3}$ ) of $\mathbf{1 2}$
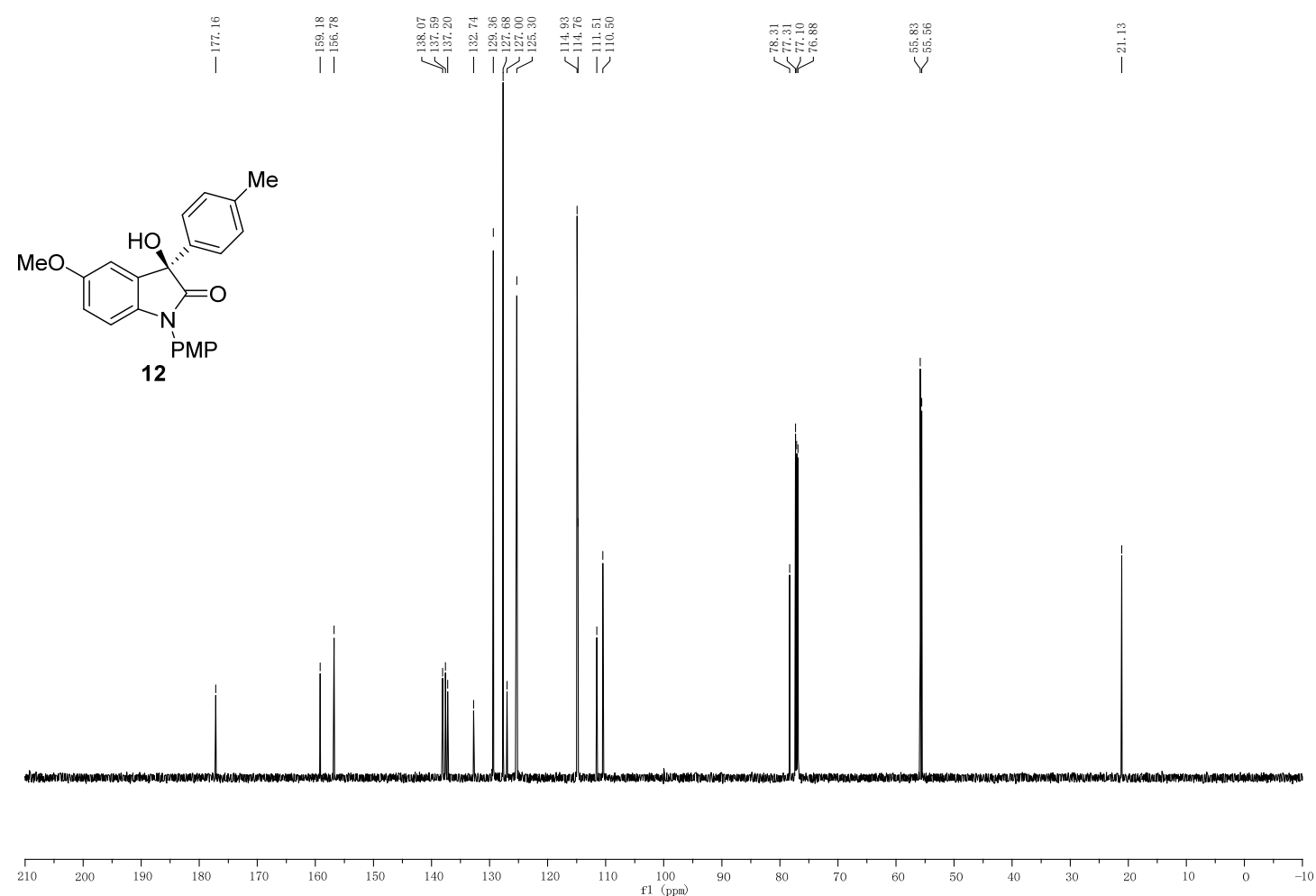
${ }^{1} \mathrm{H} \mathrm{NMR}\left(600 \mathrm{MHz}, \mathrm{CDCl}_{3}\right)$ of $\mathbf{1 3}$

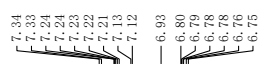

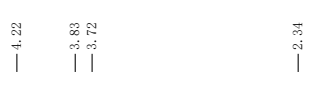

$\underbrace{\text { PMP }}_{13}$

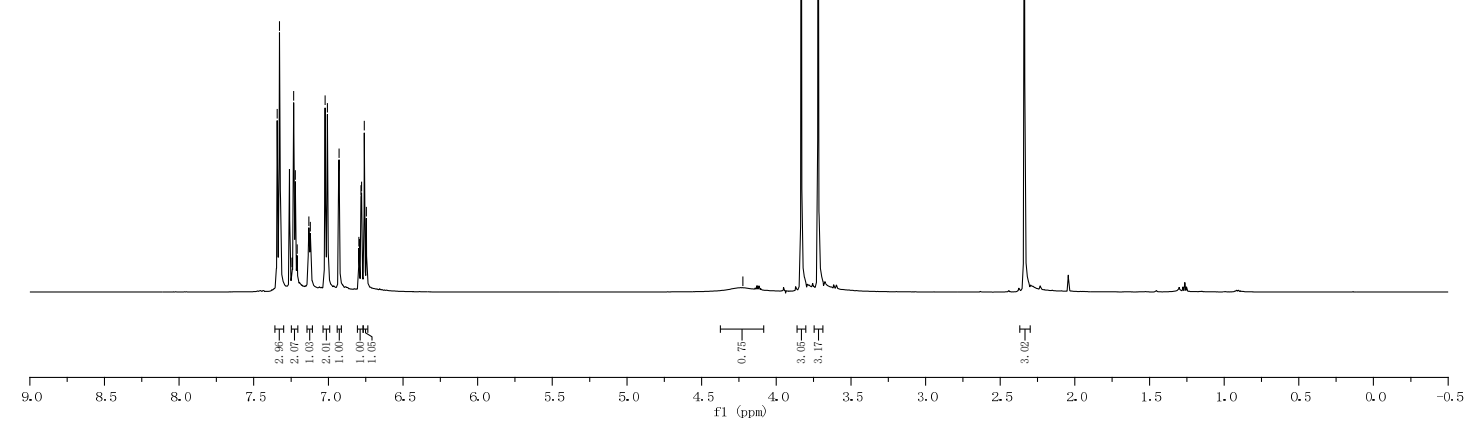

${ }^{13} \mathrm{C}$ NMR (150 MHz, $\mathrm{CDCl}_{3}$ ) of $\mathbf{1 3}$

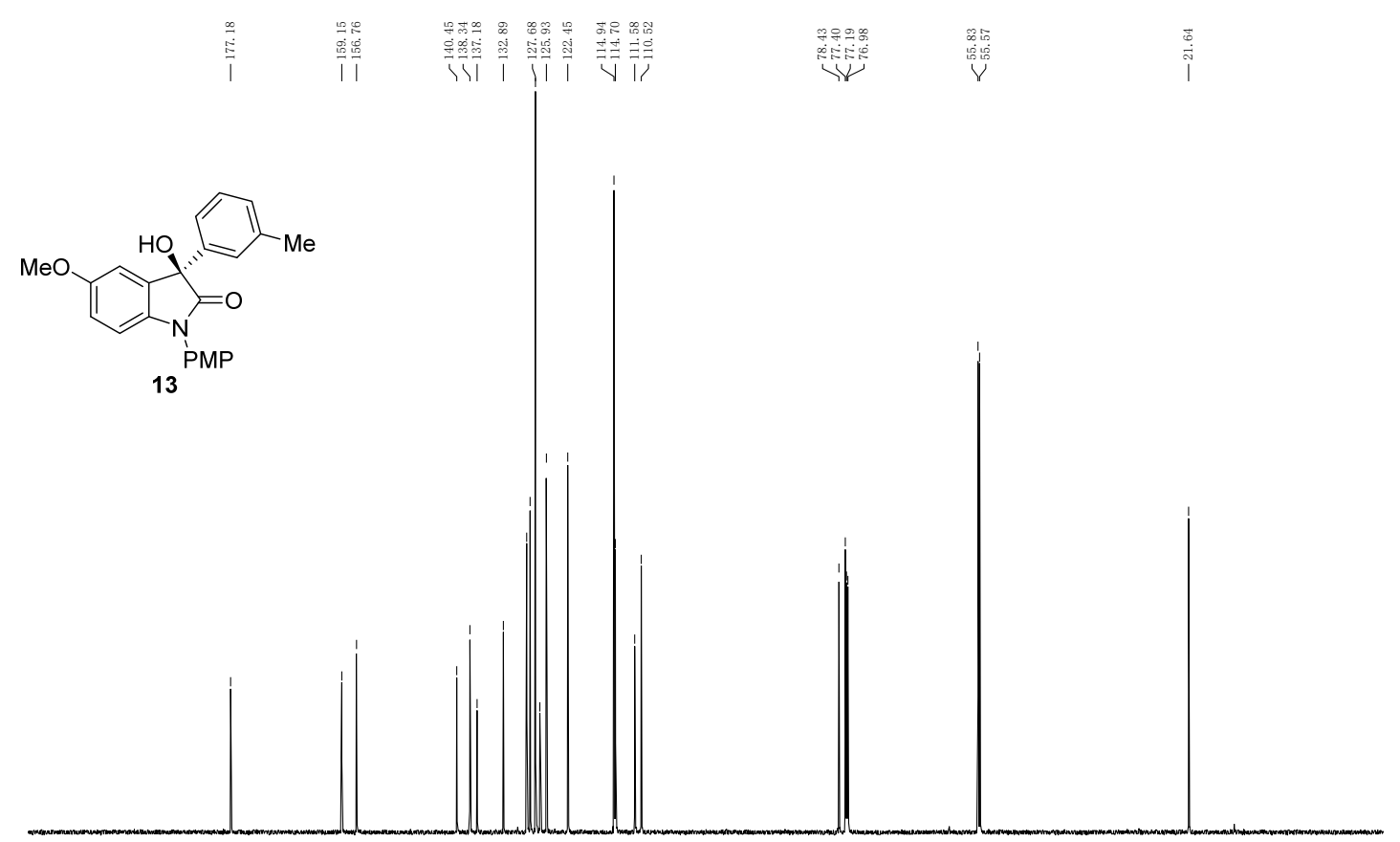

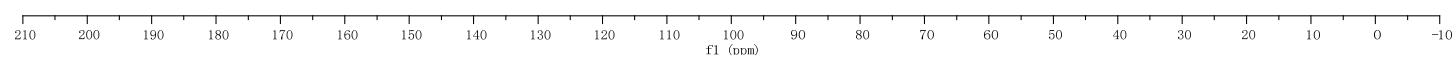


${ }^{1} \mathrm{H} \mathrm{NMR}\left(600 \mathrm{MHz}, \mathrm{CDCl}_{3}\right)$ of $\mathbf{1 4}$

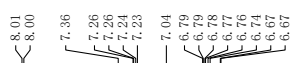

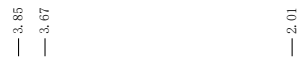
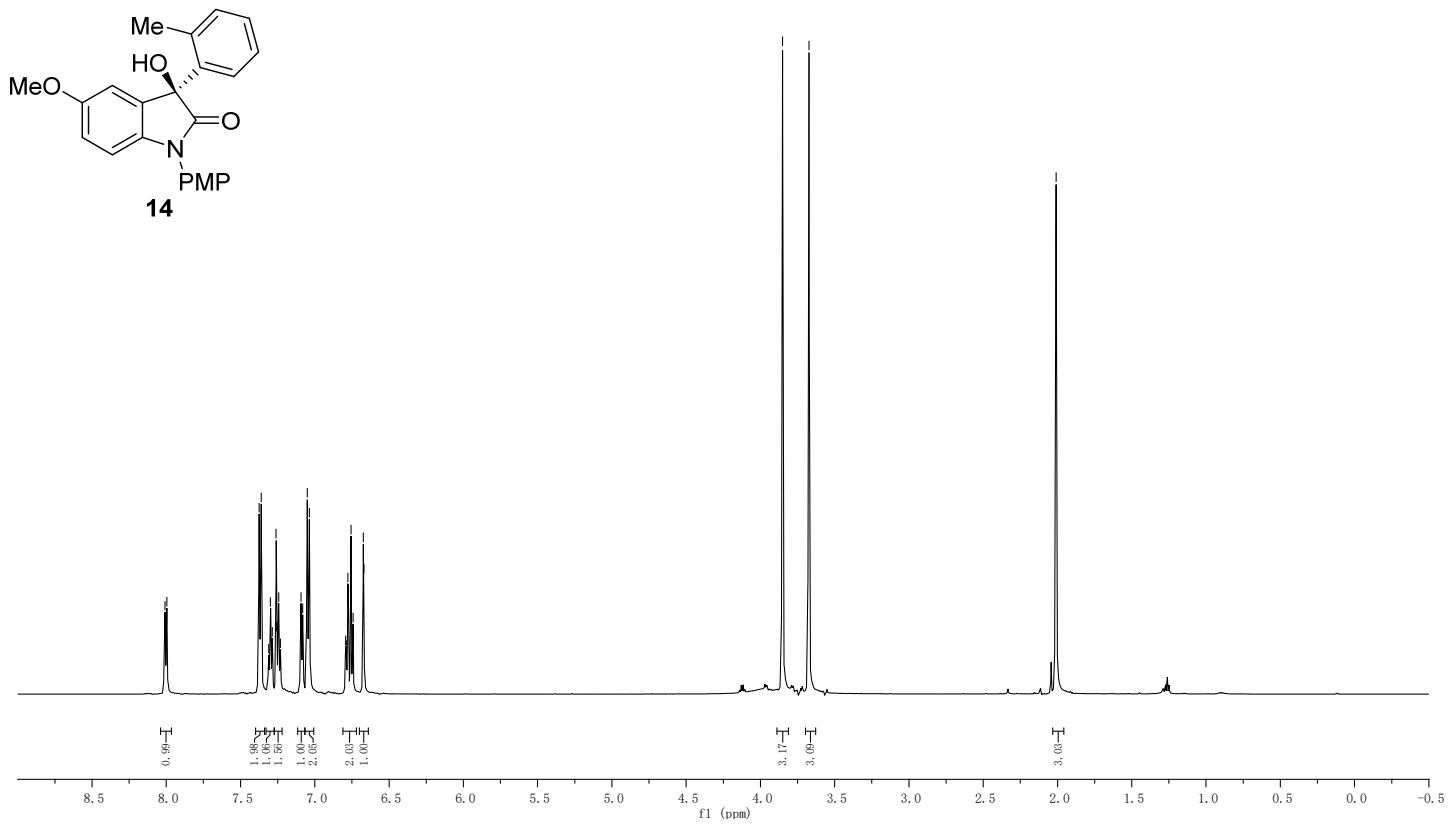

${ }^{13} \mathrm{C} \mathrm{NMR}\left(150 \mathrm{MHz}, \mathrm{CDCl}_{3}\right)$ of 14
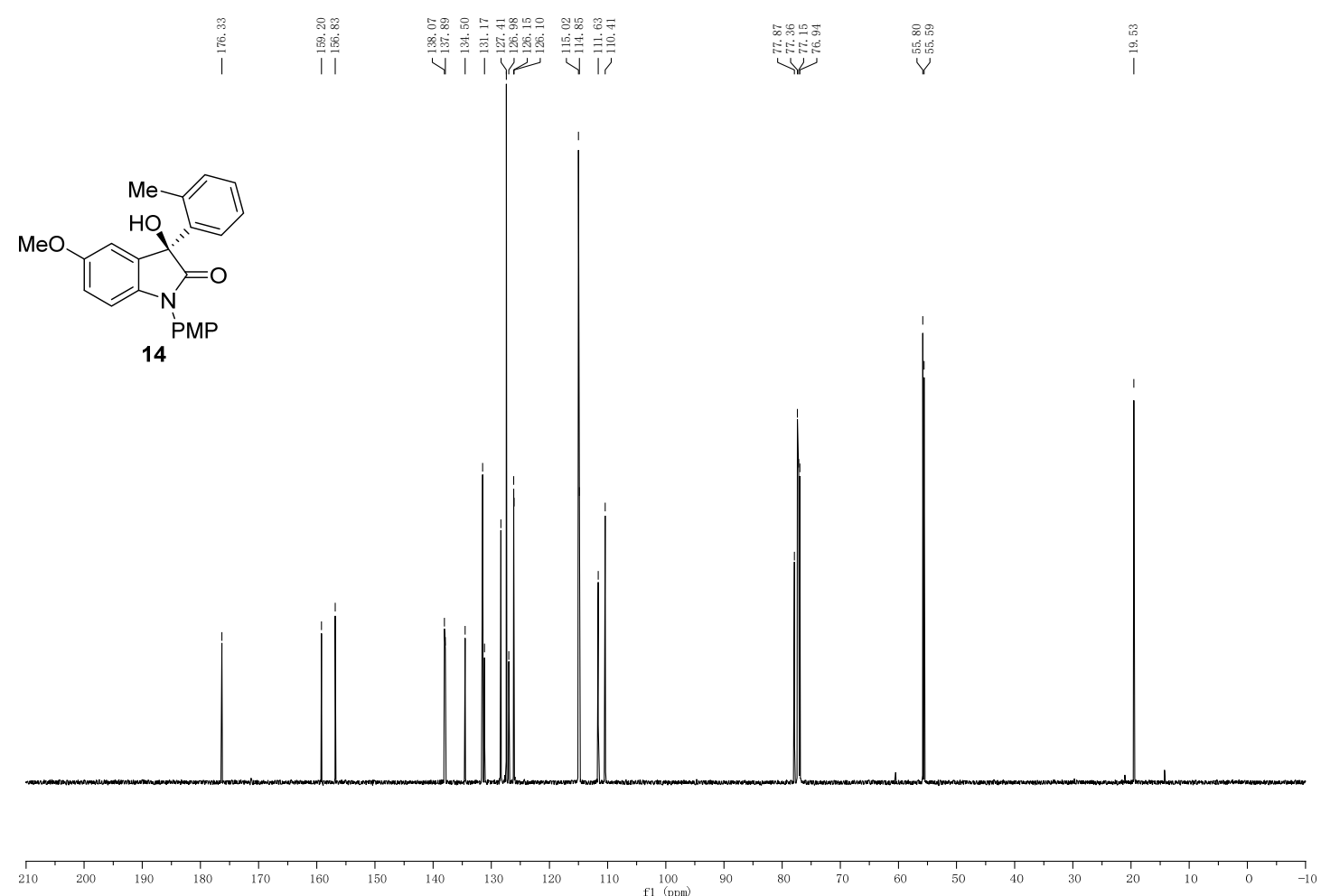
${ }^{1} \mathrm{H}$ NMR (600 MHz, $\mathrm{CDCl}_{3}$ ) of $\mathbf{1 5}$

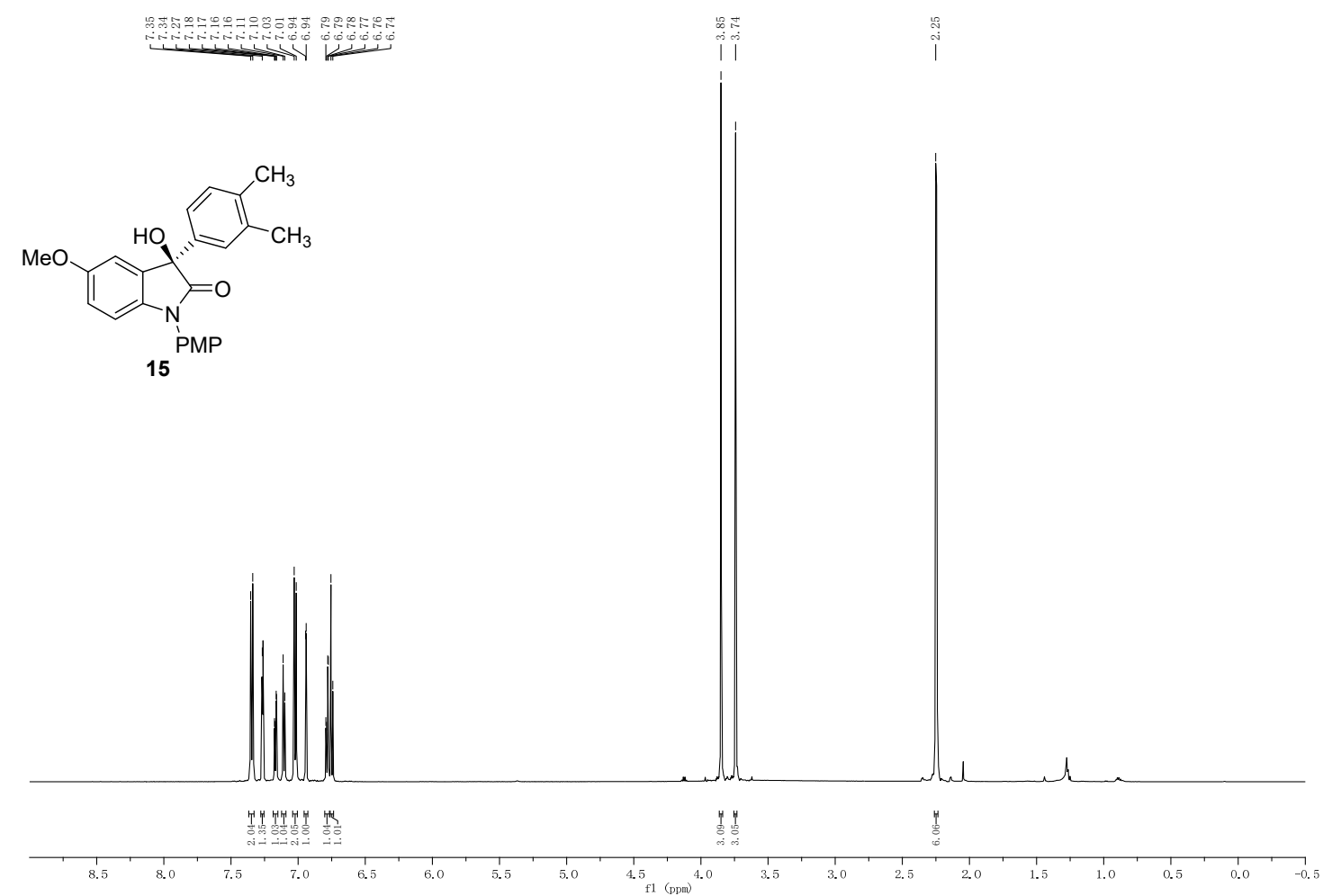

${ }^{13} \mathrm{C}$ NMR (150 MHz, $\left.\mathrm{CDCl}_{3}\right)$ of $\mathbf{1 5}$
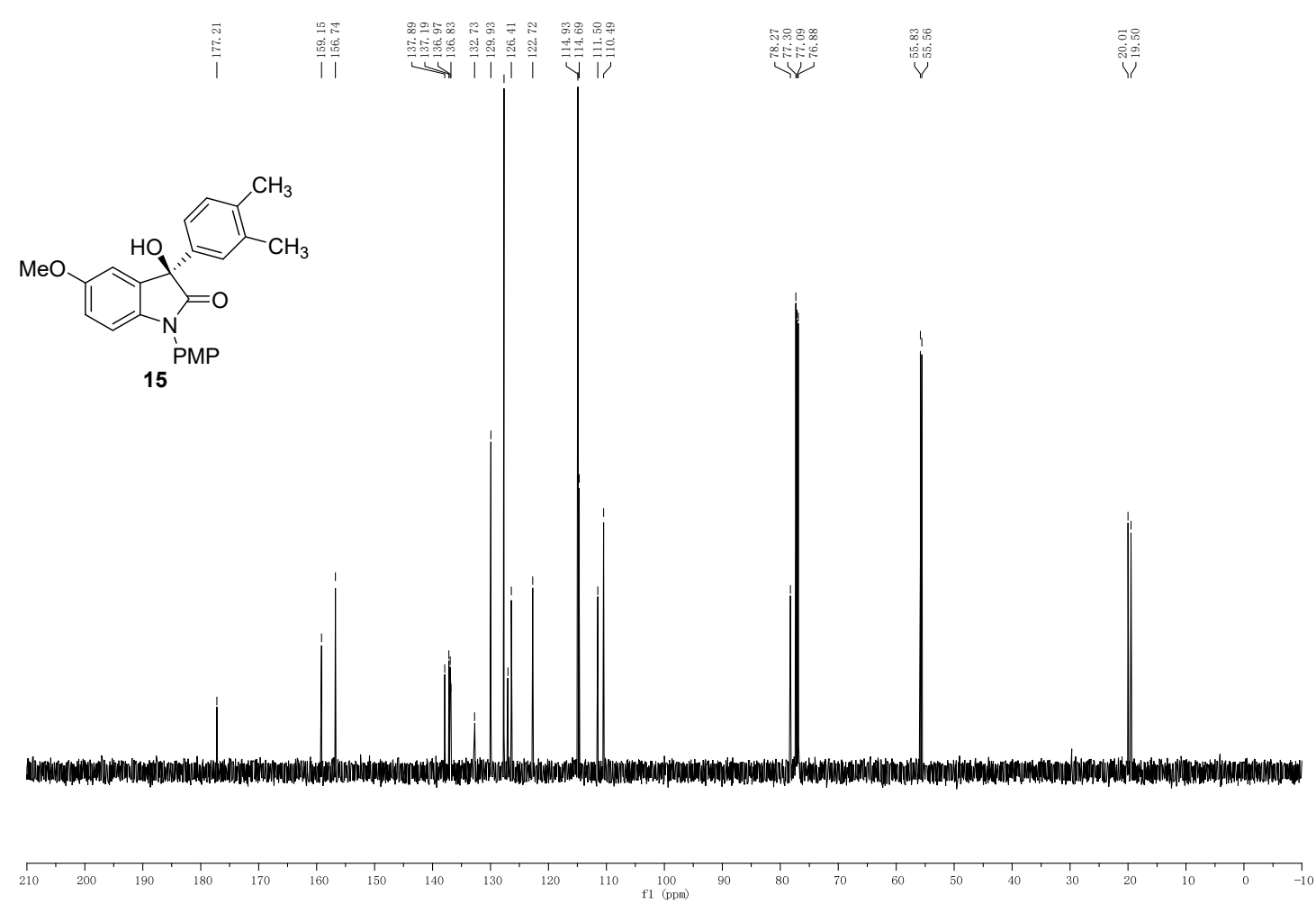
${ }^{1} \mathrm{H} \mathrm{NMR}\left(600 \mathrm{MHz}, \mathrm{CDCl}_{3}\right)$ of $\mathbf{1 6}$

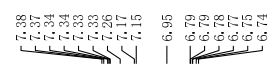
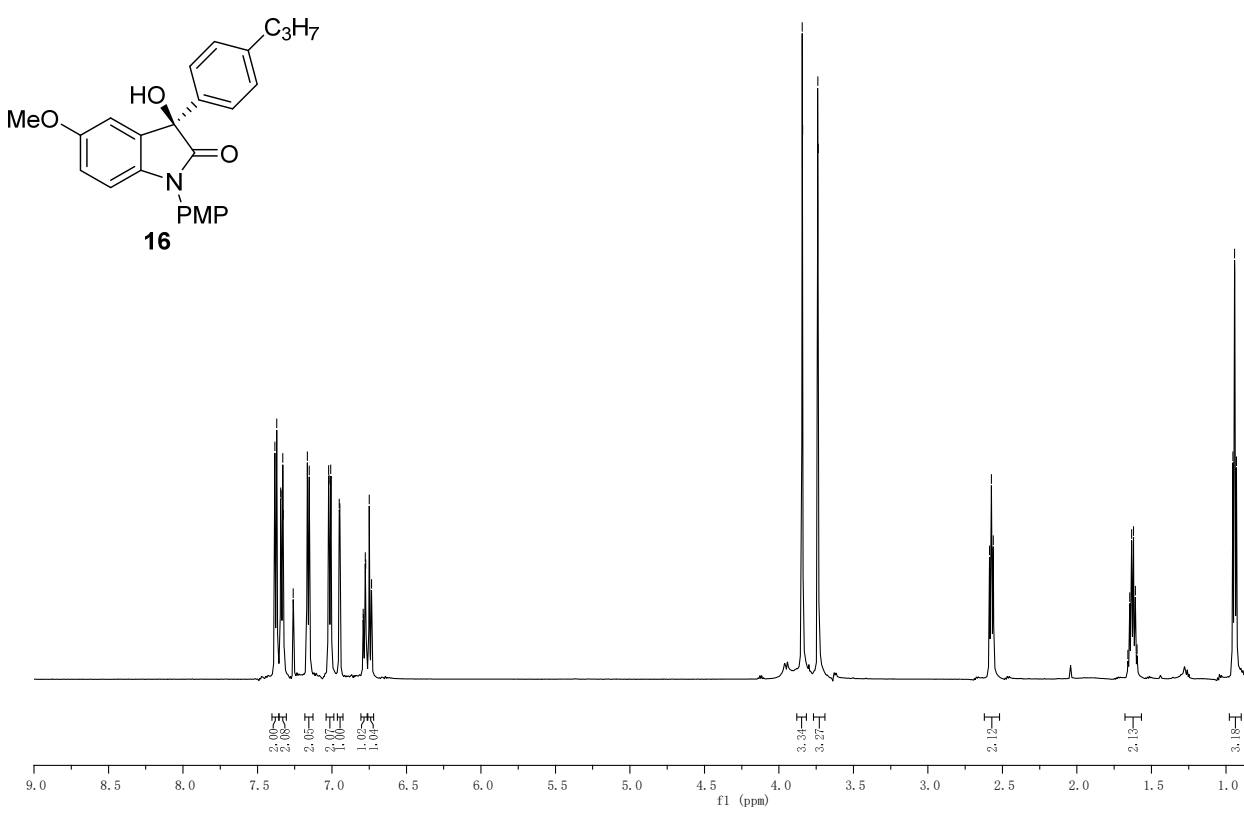

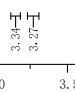

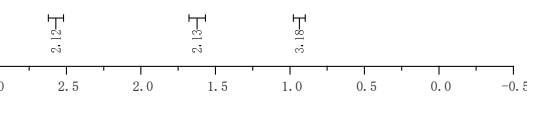

${ }^{13} \mathrm{C}$ NMR $\left(150 \mathrm{MHz}, \mathrm{CDCl}_{3}\right)$ of $\mathbf{1 6}$

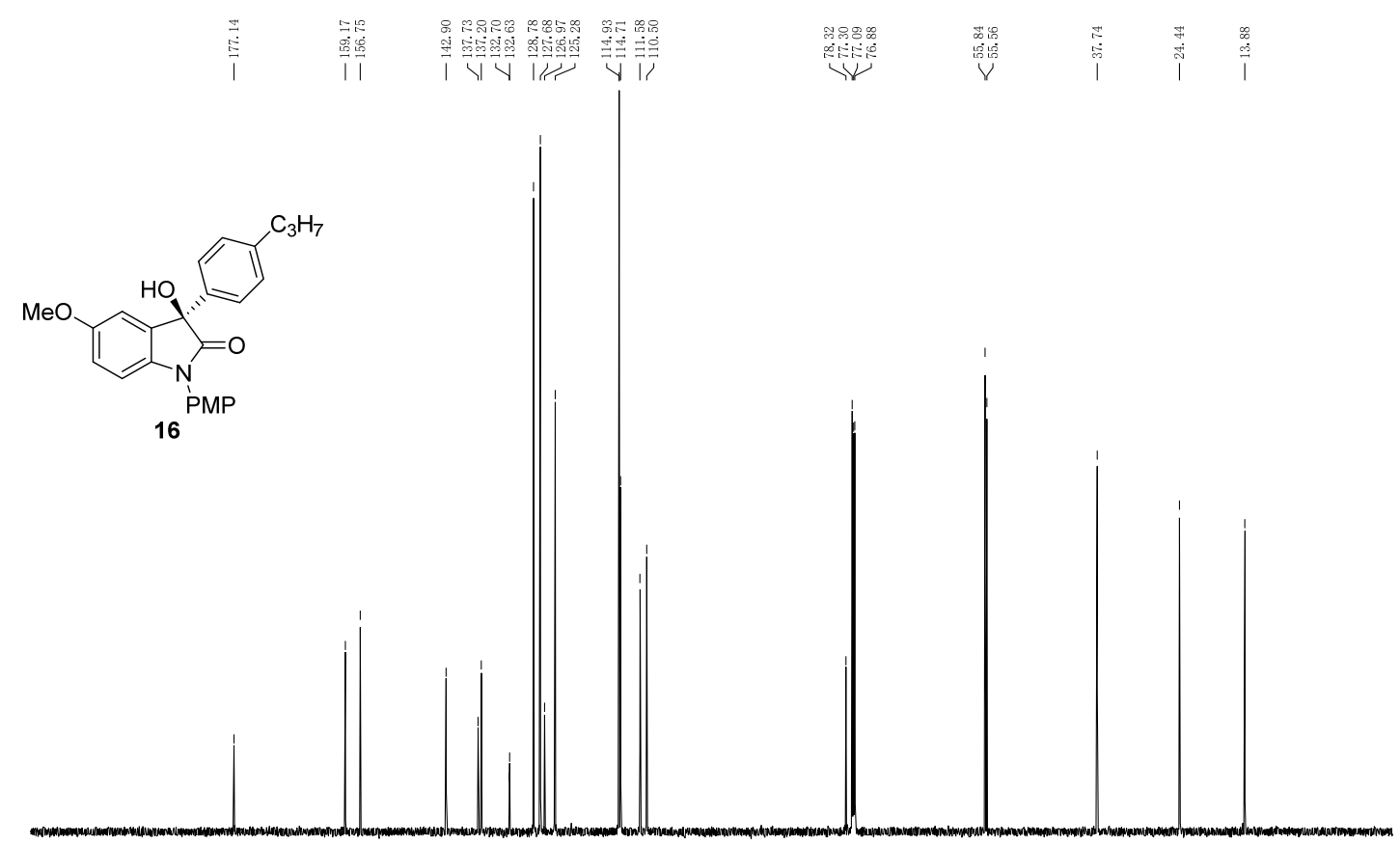

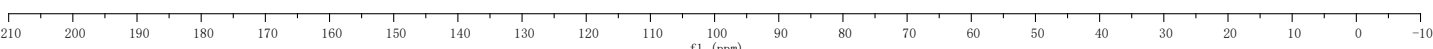


${ }^{1} \mathrm{H} \mathrm{NMR}\left(600 \mathrm{MHz}, \mathrm{CDCl}_{3}\right)$ of $\mathbf{1 7}$

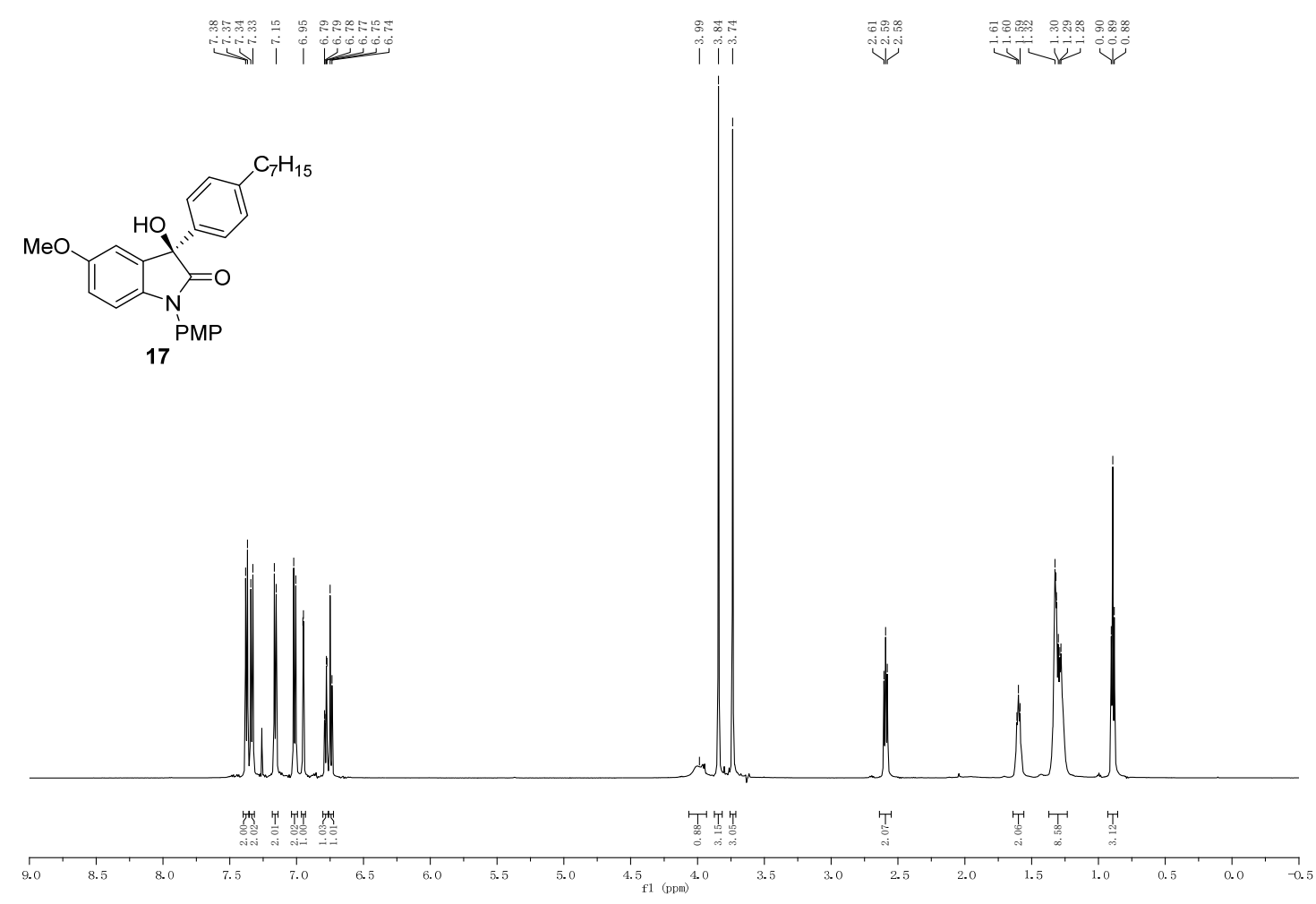

${ }^{13} \mathrm{C}$ NMR $\left(150 \mathrm{MHz}, \mathrm{CDCl}_{3}\right)$ of $\mathbf{1 7}$

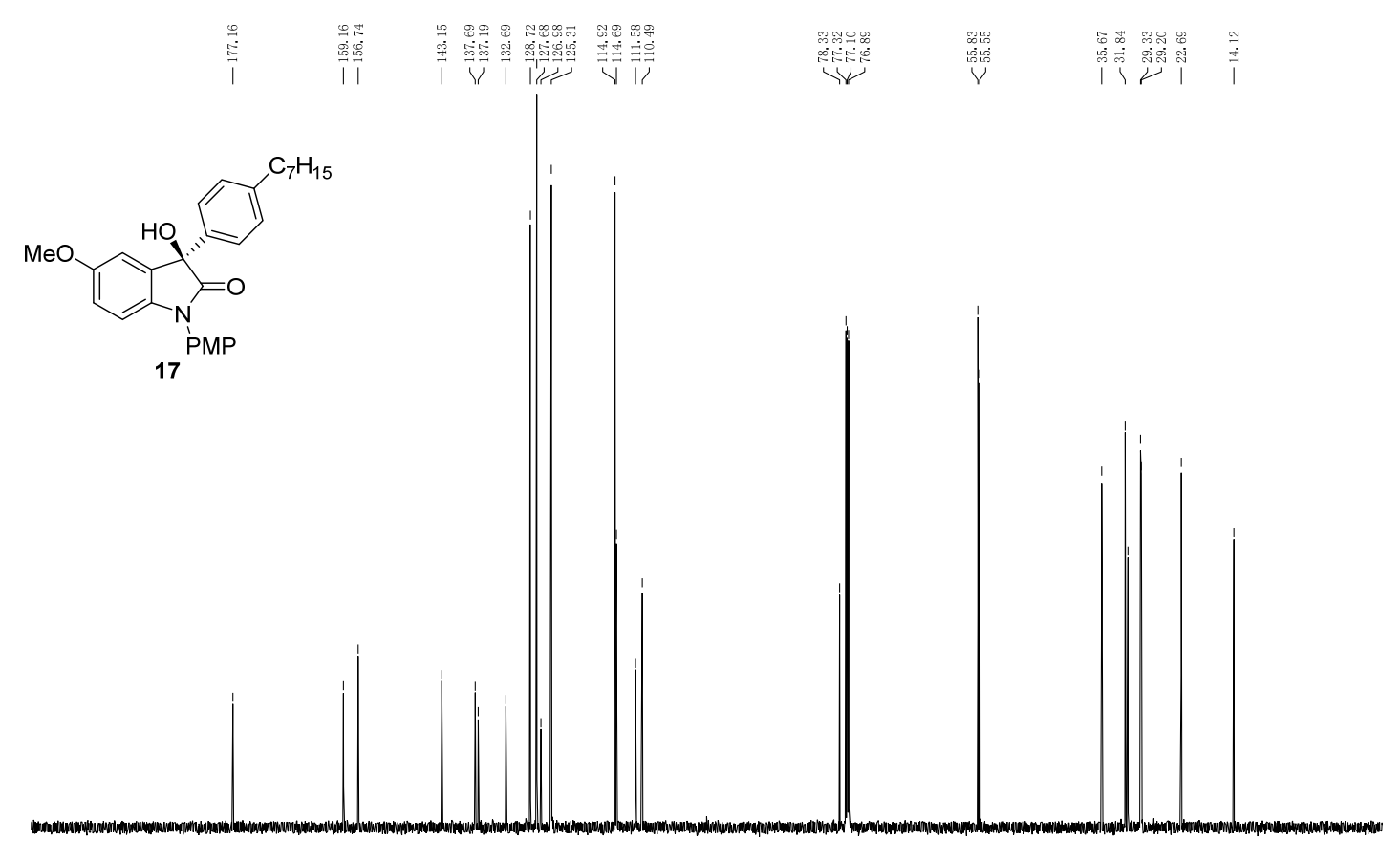

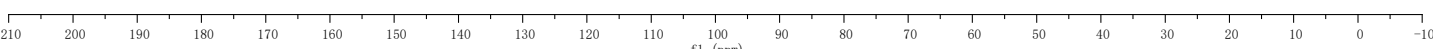


${ }^{1} \mathrm{H}$ NMR (600 MHz, MeOD) of $\mathbf{1 8}$

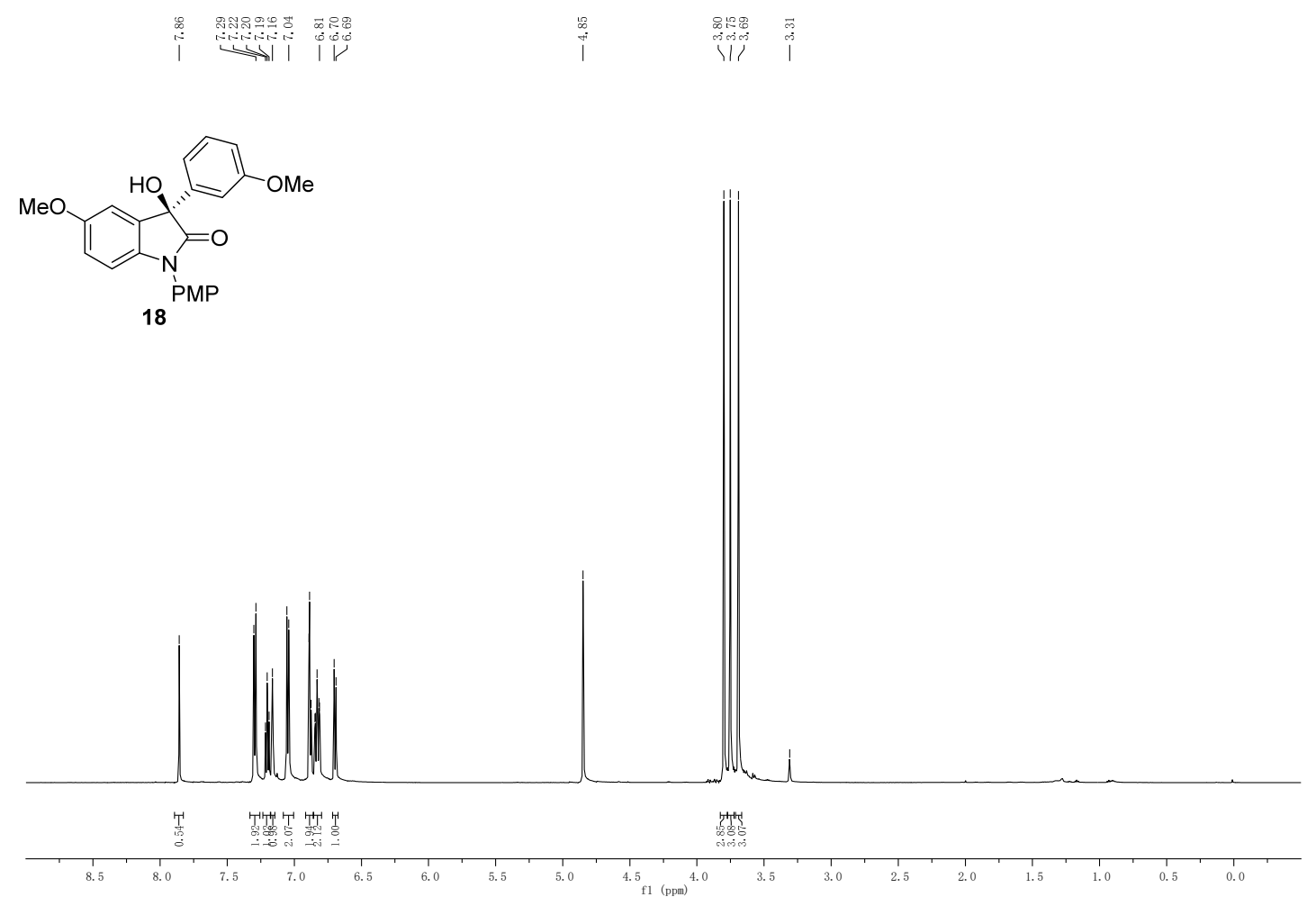

${ }^{13} \mathrm{C}$ NMR $(150 \mathrm{MHz}, \mathrm{MeOD})$ of $\mathbf{1 8}$

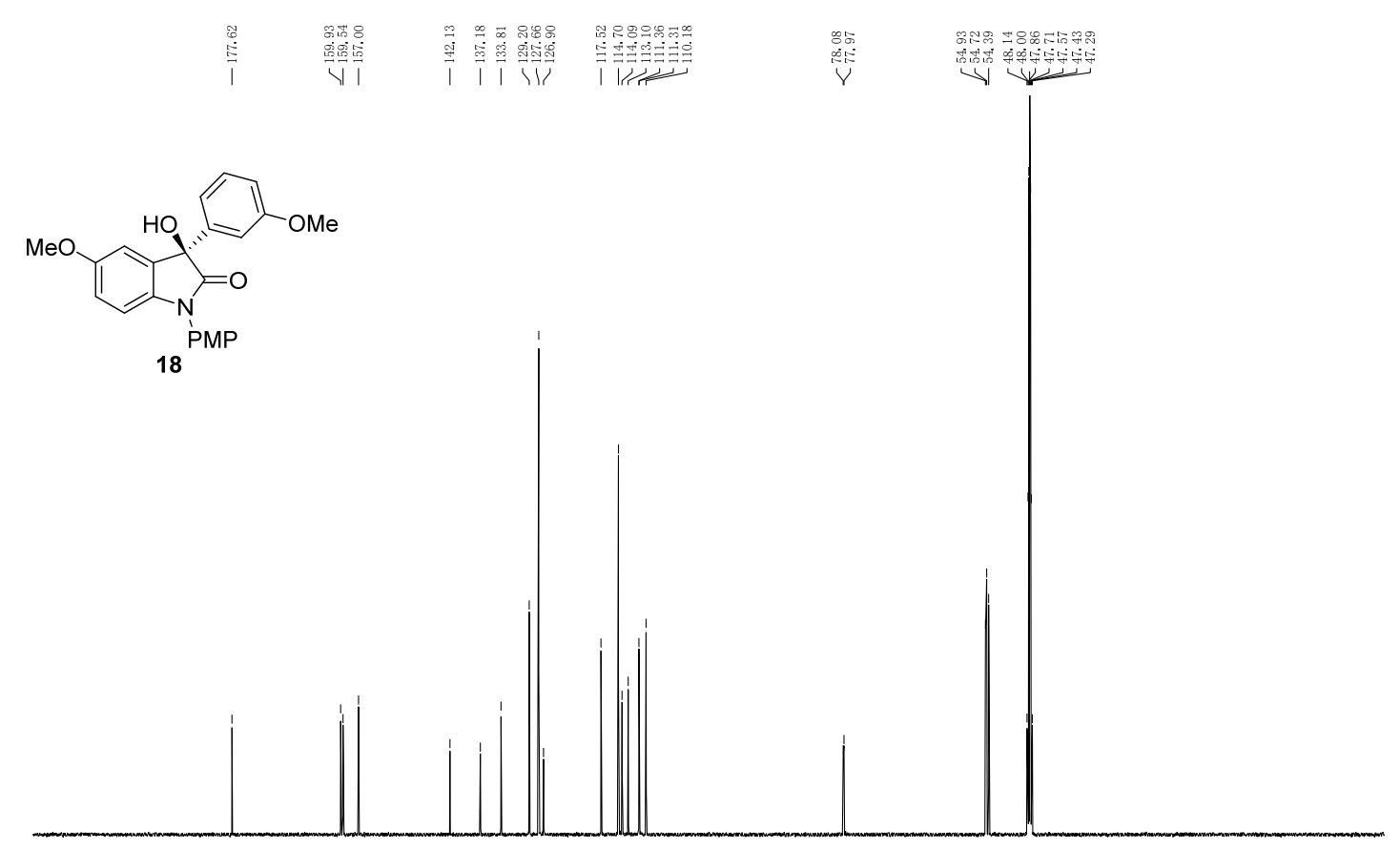

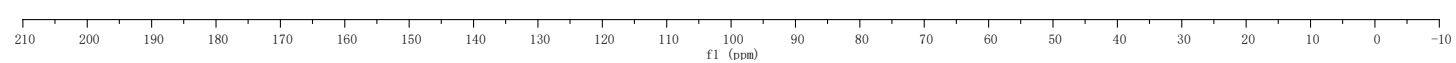


${ }^{1} \mathrm{H}$ NMR (600 MHz, $\mathrm{CDCl}_{3}$ ) of 19

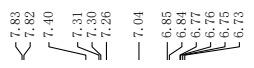
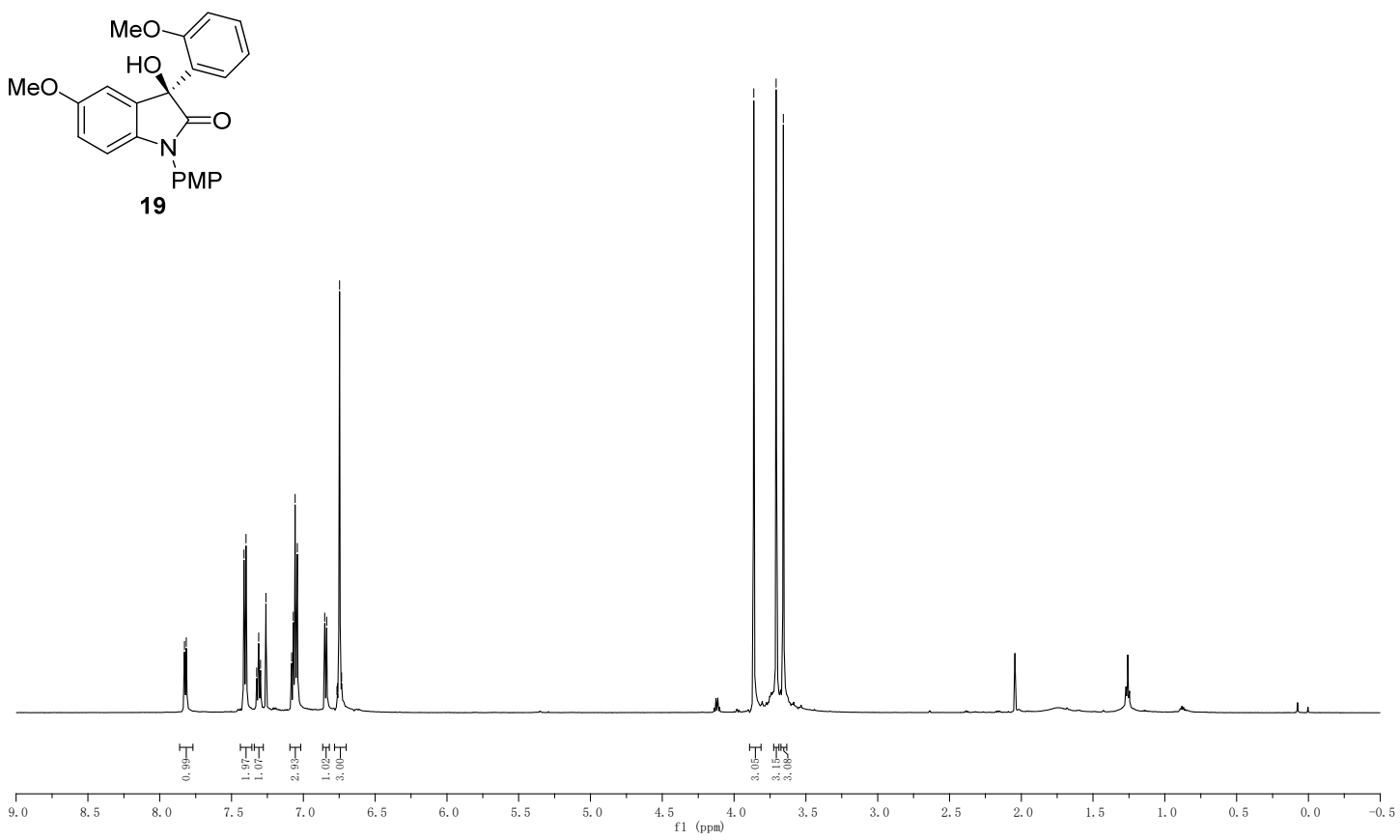

${ }^{13} \mathrm{C}$ NMR (150 MHz, $\mathrm{CDCl}_{3}$ ) of 19
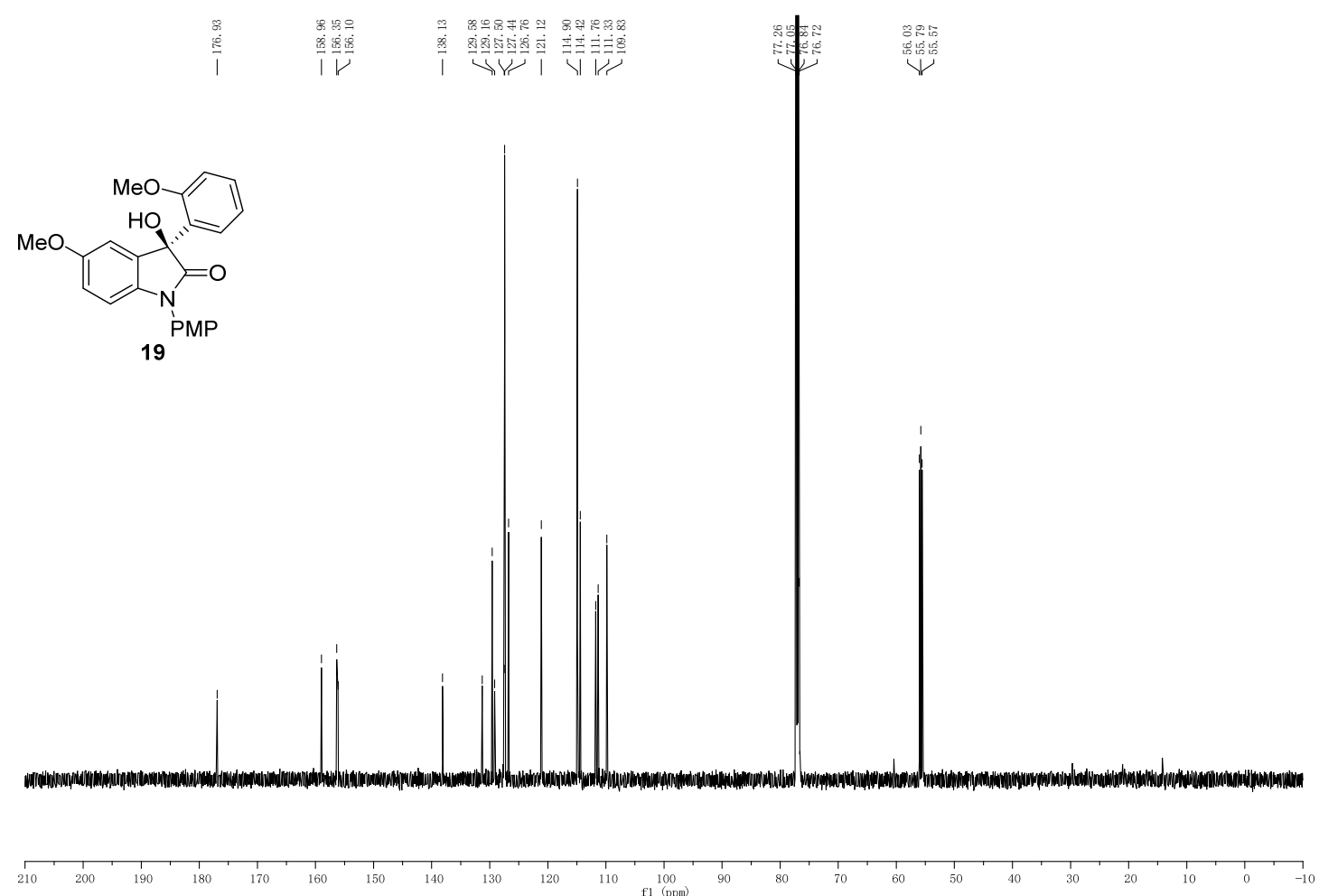
${ }^{1} \mathrm{H}$ NMR $\left(600 \mathrm{MHz}, \mathrm{CDCl}_{3}\right)$ of $\mathbf{2 0}$

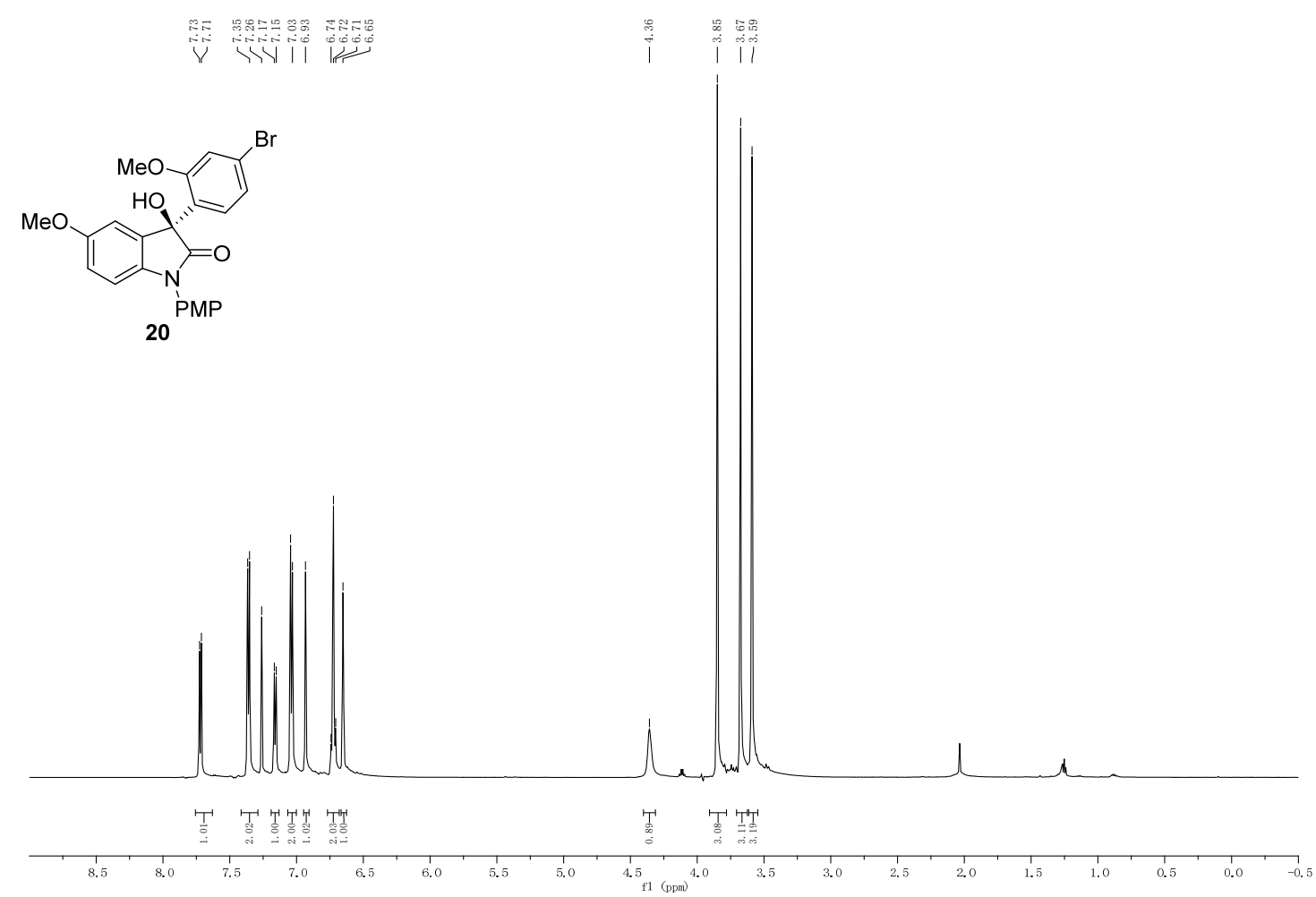

${ }^{13} \mathrm{C}$ NMR $\left(150 \mathrm{MHz}, \mathrm{CDCl}_{3}\right)$ of 20
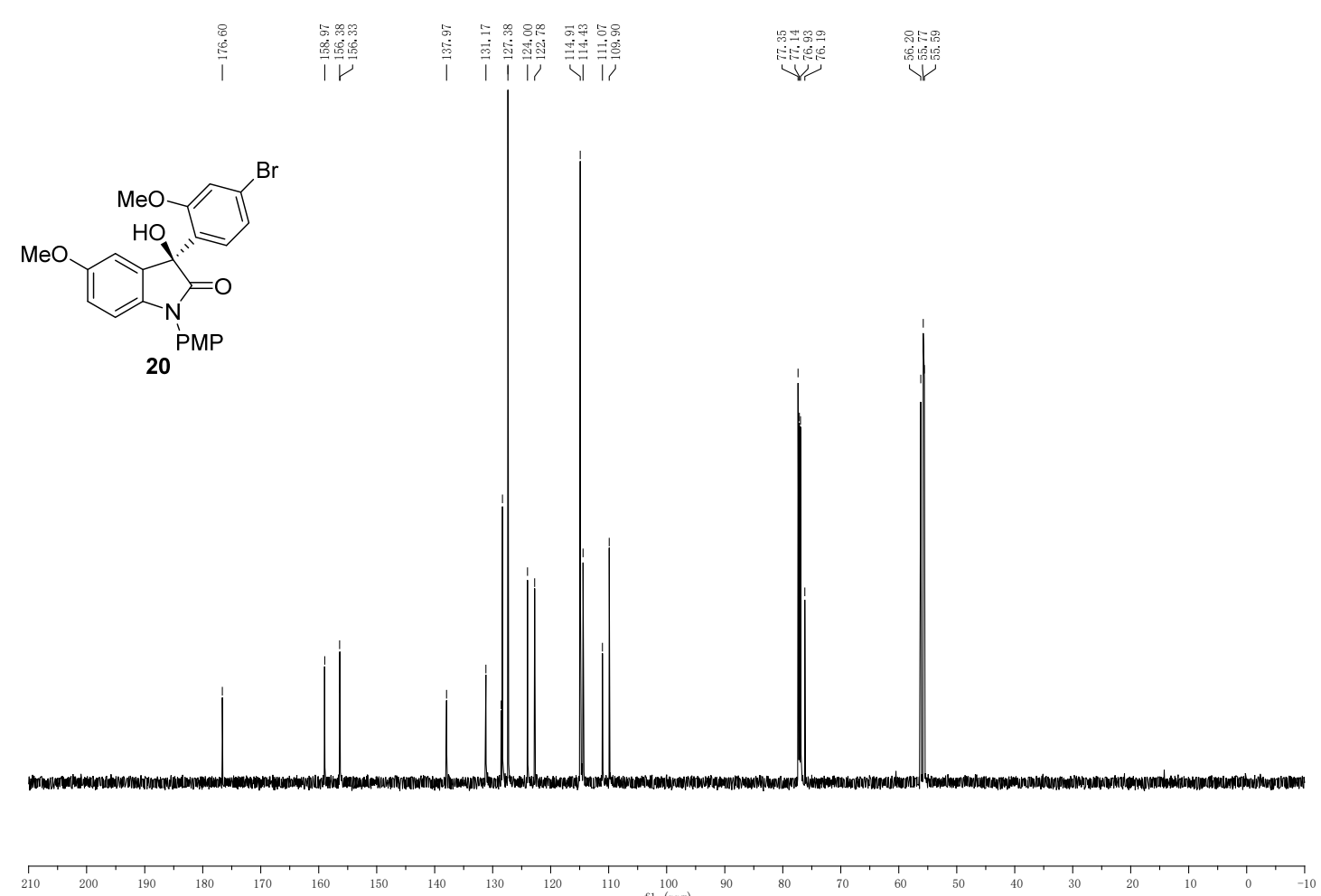
${ }^{1} \mathrm{H}$ NMR (600 MHz, DMSO) of 21

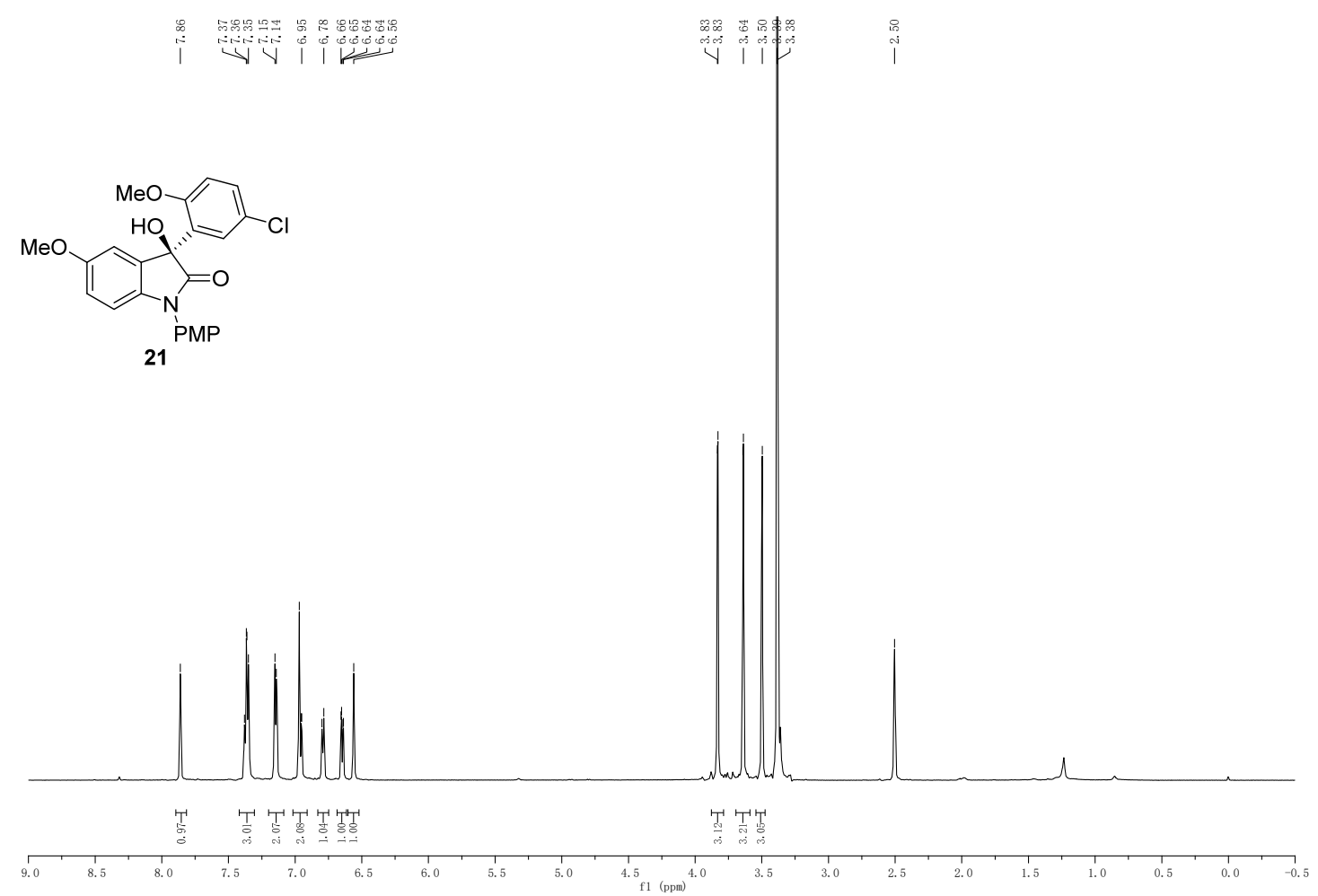

${ }^{13} \mathrm{C}$ NMR (150 MHz, DMSO) of 21

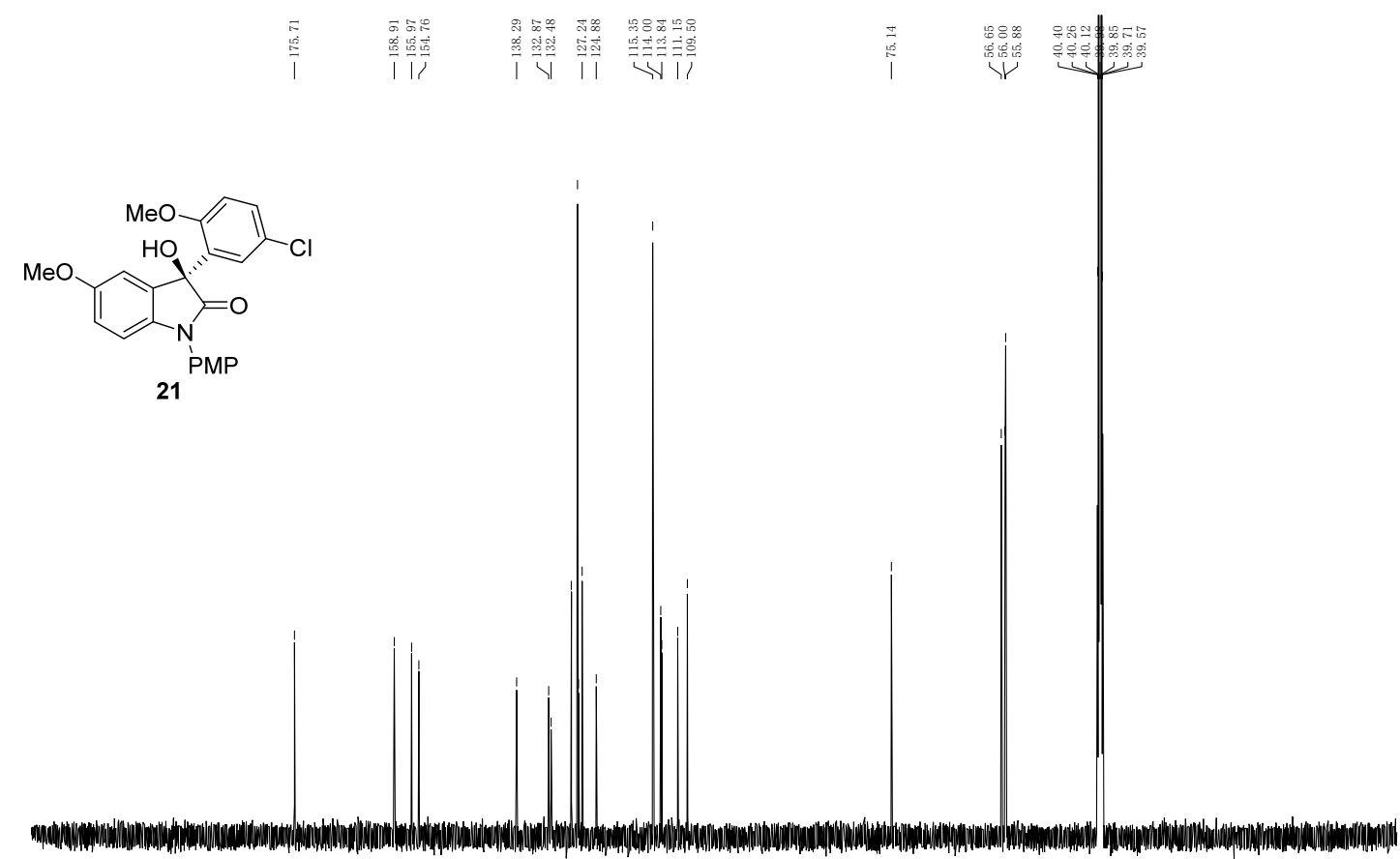

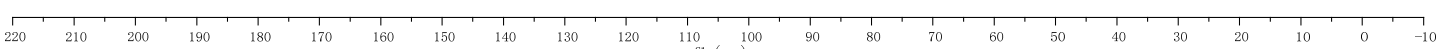


${ }^{1} \mathrm{H}$ NMR (600 MHz, $\mathrm{CDCl}_{3}$ ) of 22

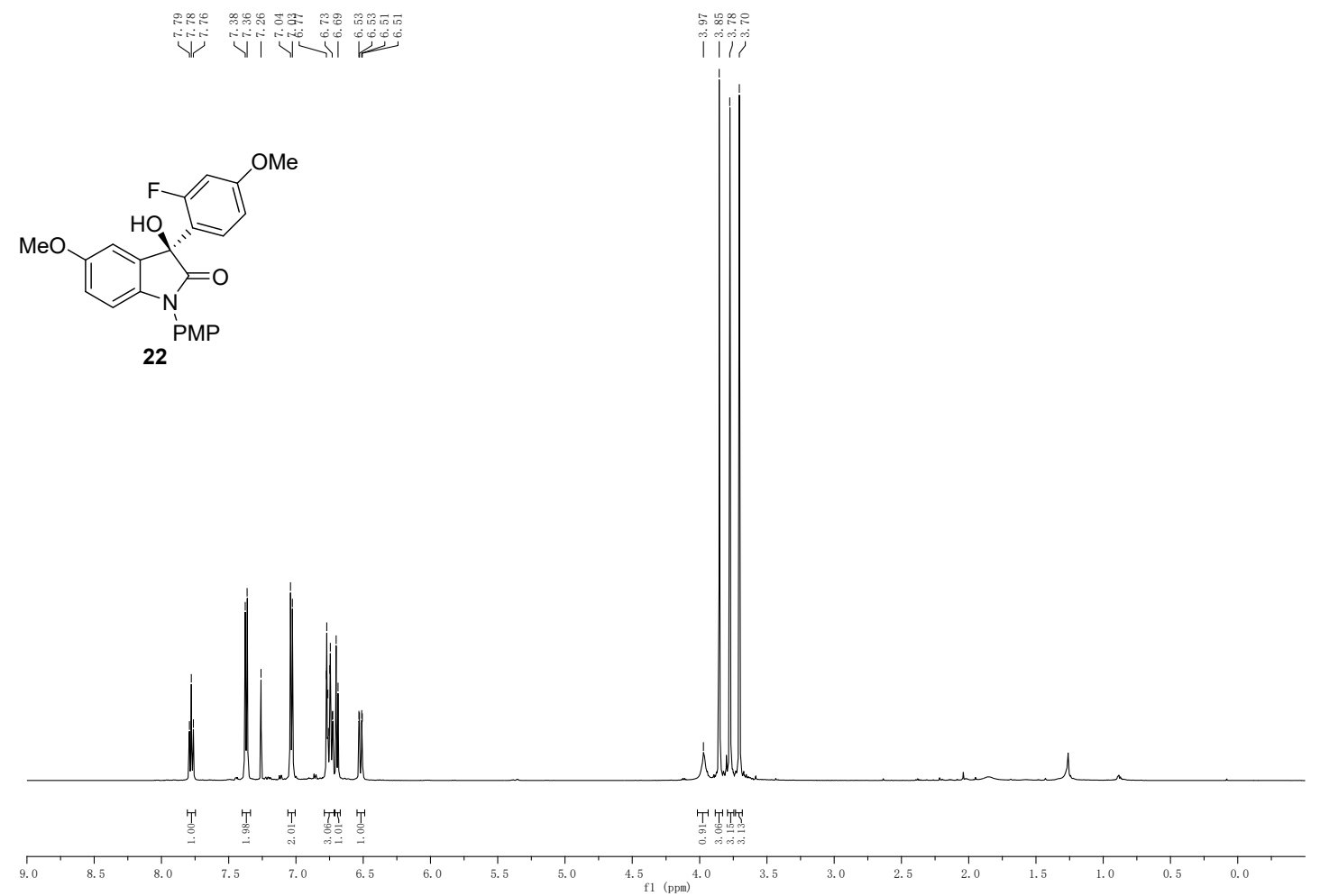

${ }^{13} \mathrm{C}$ NMR $\left(150 \mathrm{MHz}, \mathrm{CDCl}_{3}\right)$ of 22

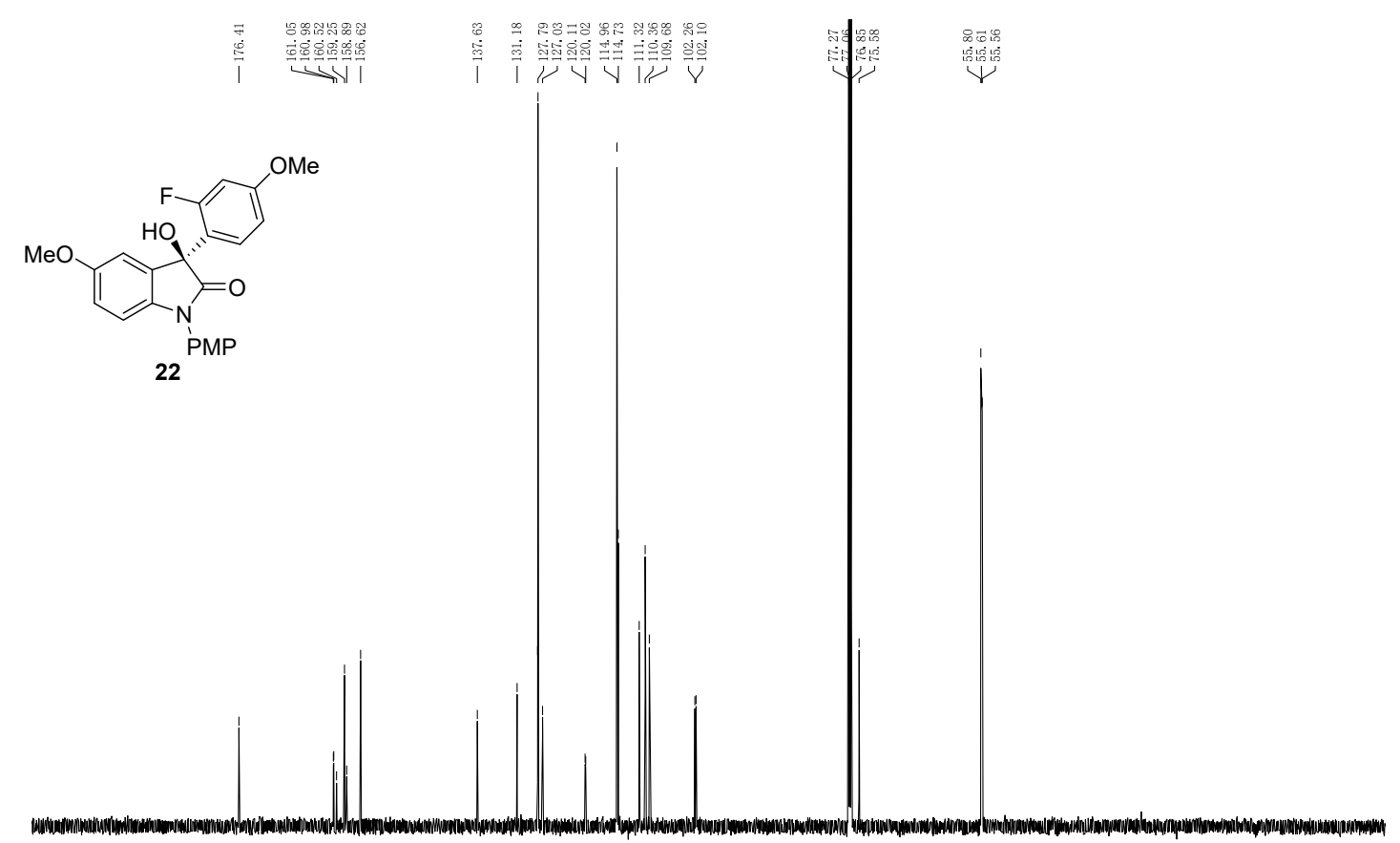

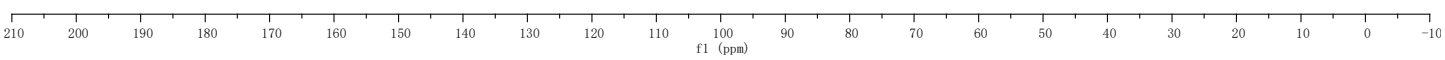


${ }^{19} \mathrm{~F}$ NMR $\left(565 \mathrm{MHz}, \mathrm{CDCl}_{3}\right)$ of 22

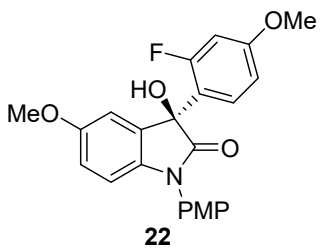

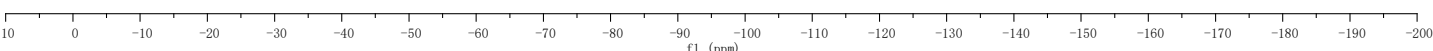


${ }^{1} \mathrm{H}$ NMR (600 MHz, $\mathrm{CDCl}_{3}$ ) of $\mathbf{2 3}$

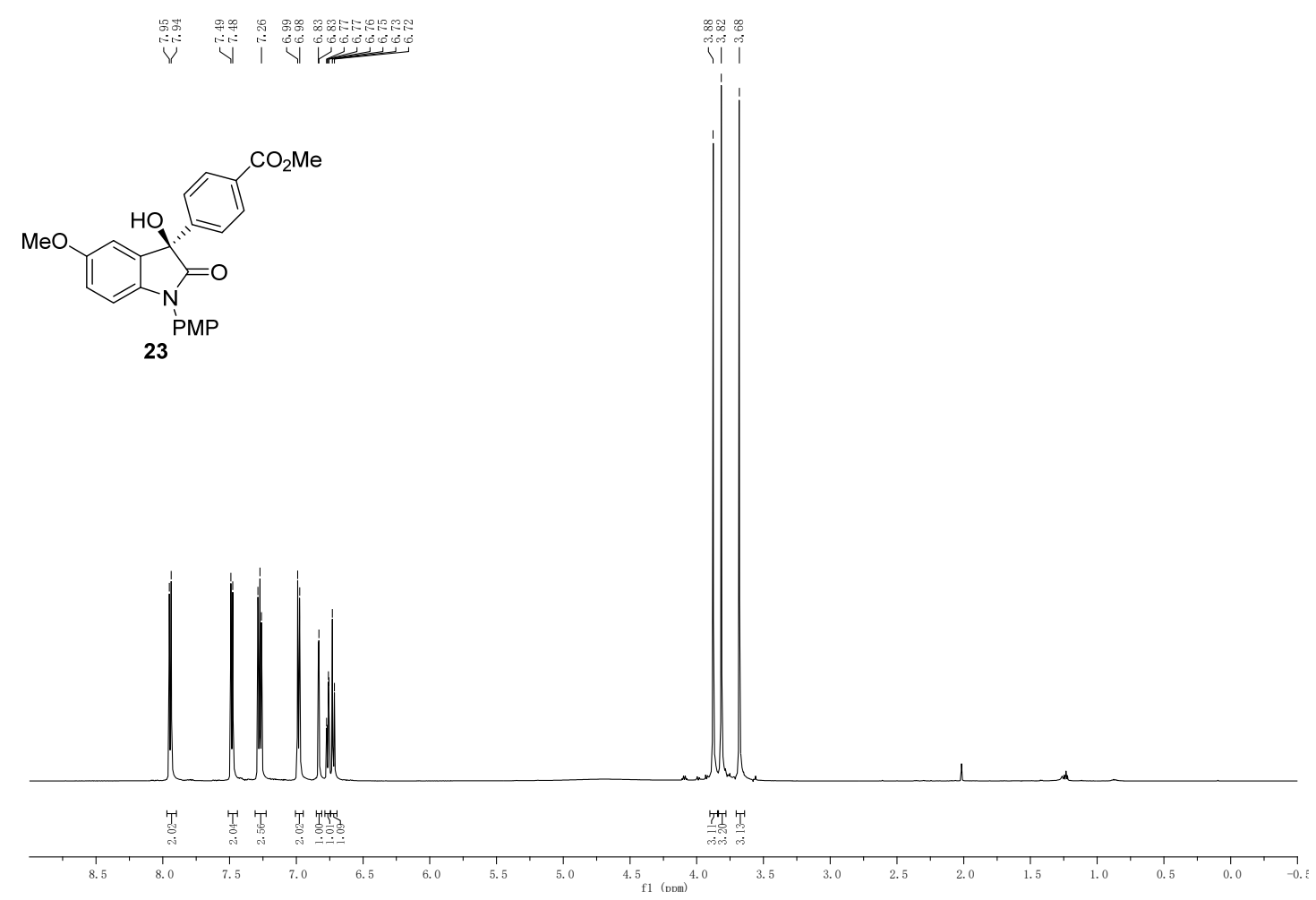

${ }^{13} \mathrm{C}$ NMR (150 MHz, $\mathrm{CDCl}_{3}$ ) of $\mathbf{2 3}$

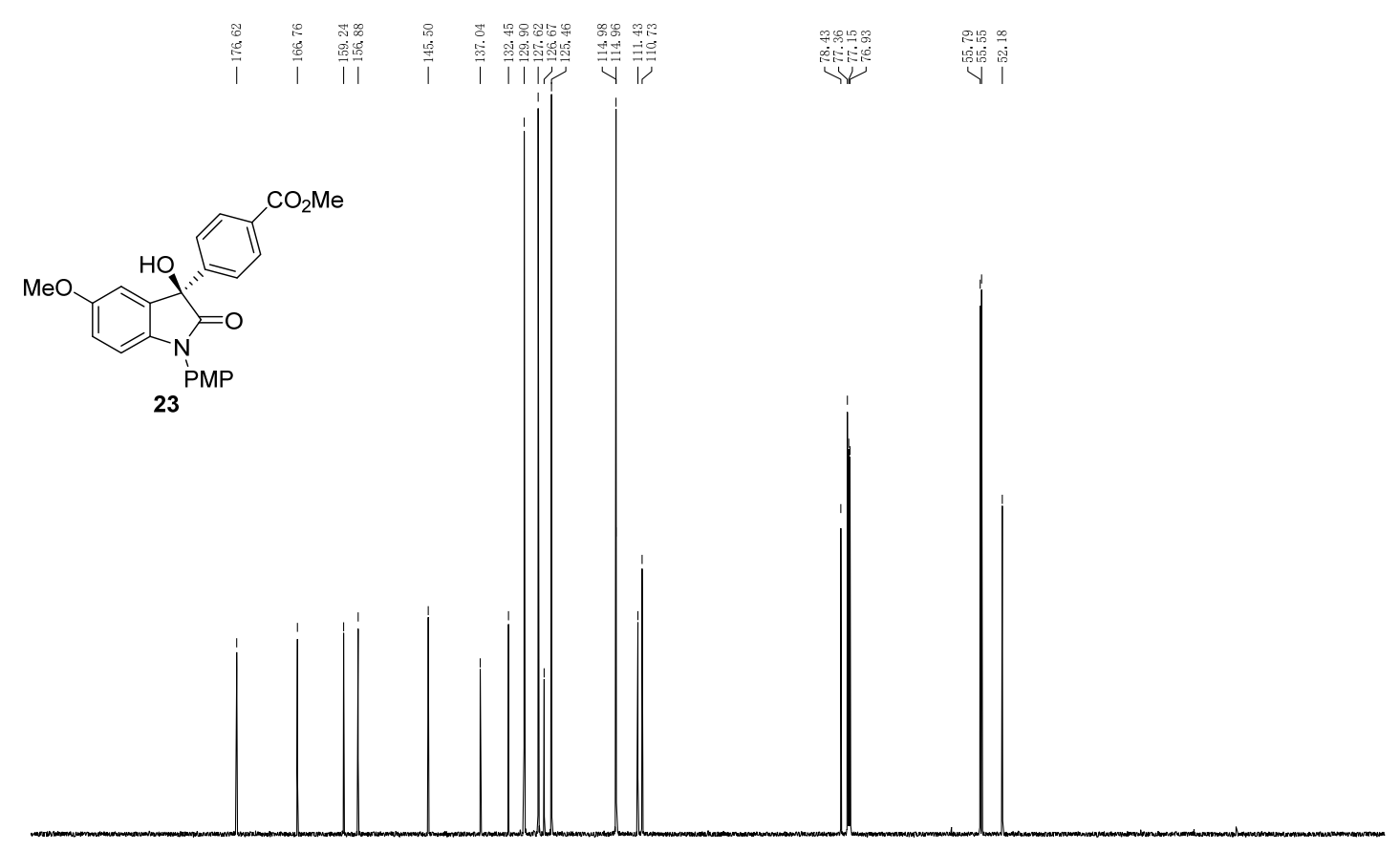




${ }^{1} \mathrm{H}$ NMR $\left(600 \mathrm{MHz}, \mathrm{CDCl}_{3}\right)$ of $\mathbf{2 4}$

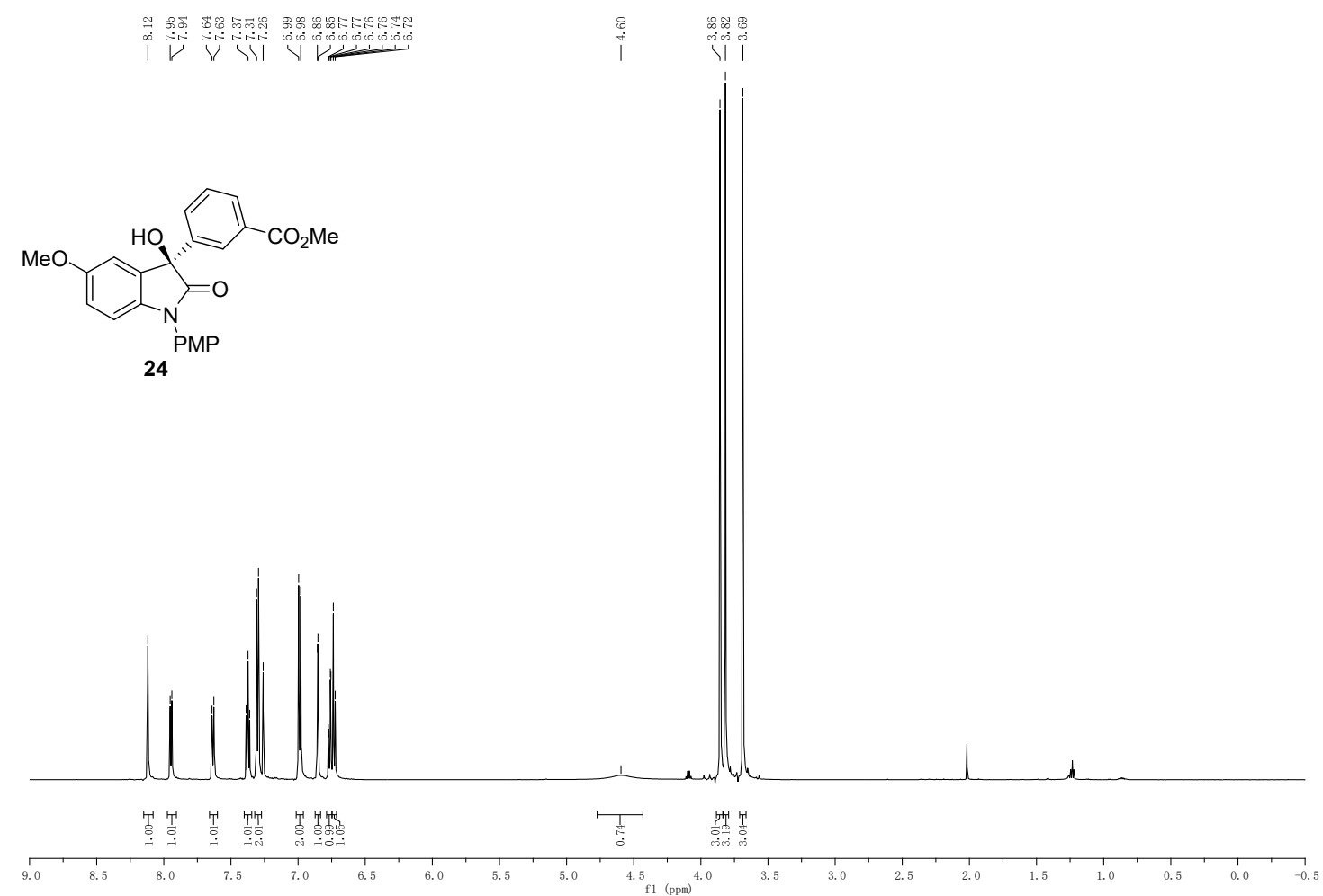

${ }^{13} \mathrm{C}$ NMR $\left(150 \mathrm{MHz}, \mathrm{CDCl}_{3}\right)$ of 24

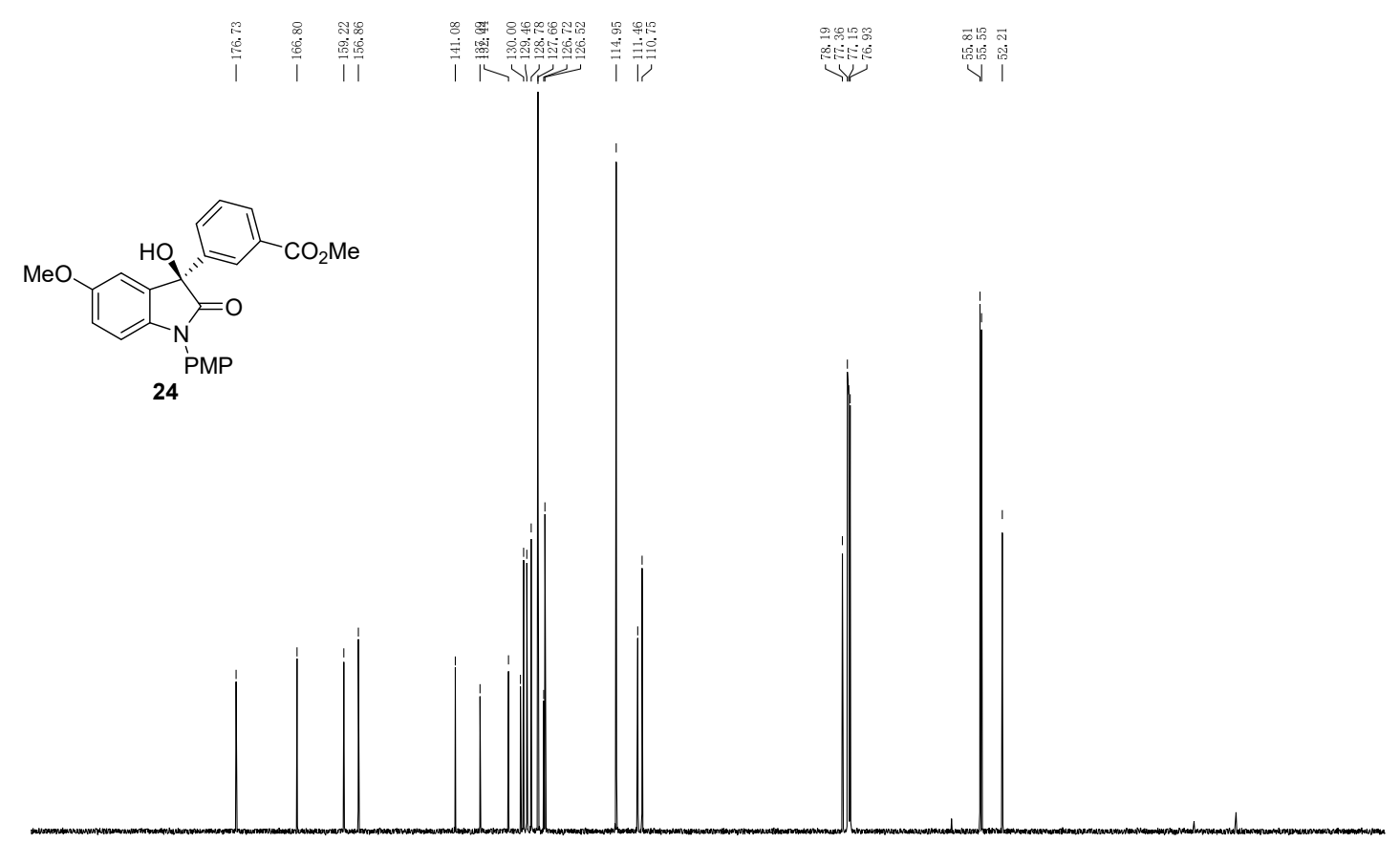

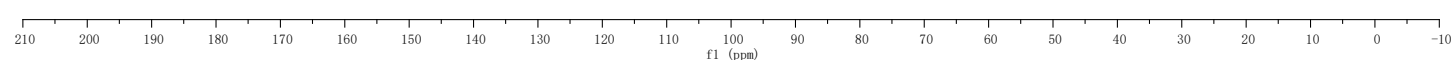


${ }^{1} \mathrm{H}$ NMR (600 MHz, $\mathrm{CDCl}_{3}$ ) of $\mathbf{2 5}$

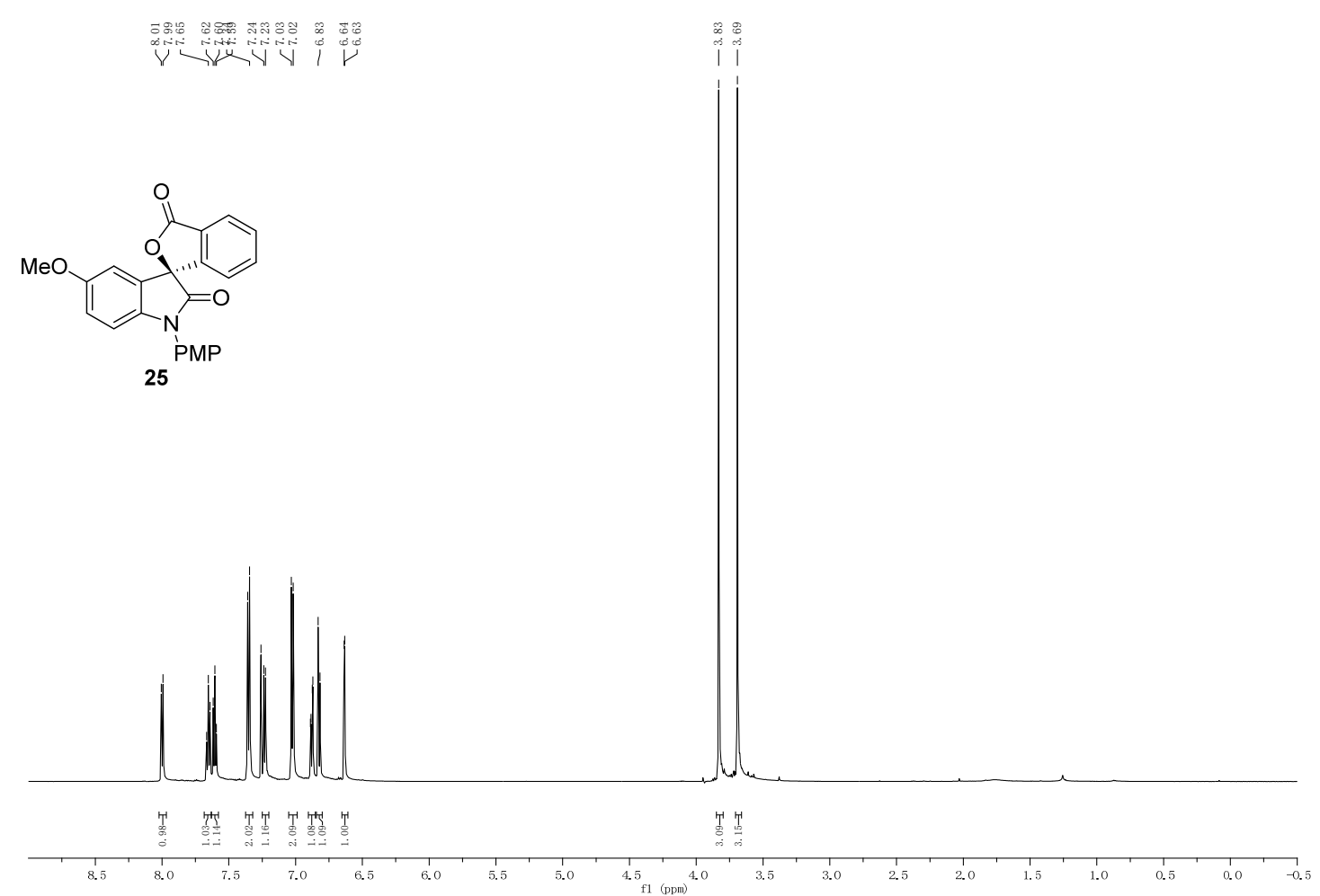

${ }^{13} \mathrm{C}$ NMR (150 MHz, $\mathrm{CDCl}_{3}$ ) of $\mathbf{2 5}$
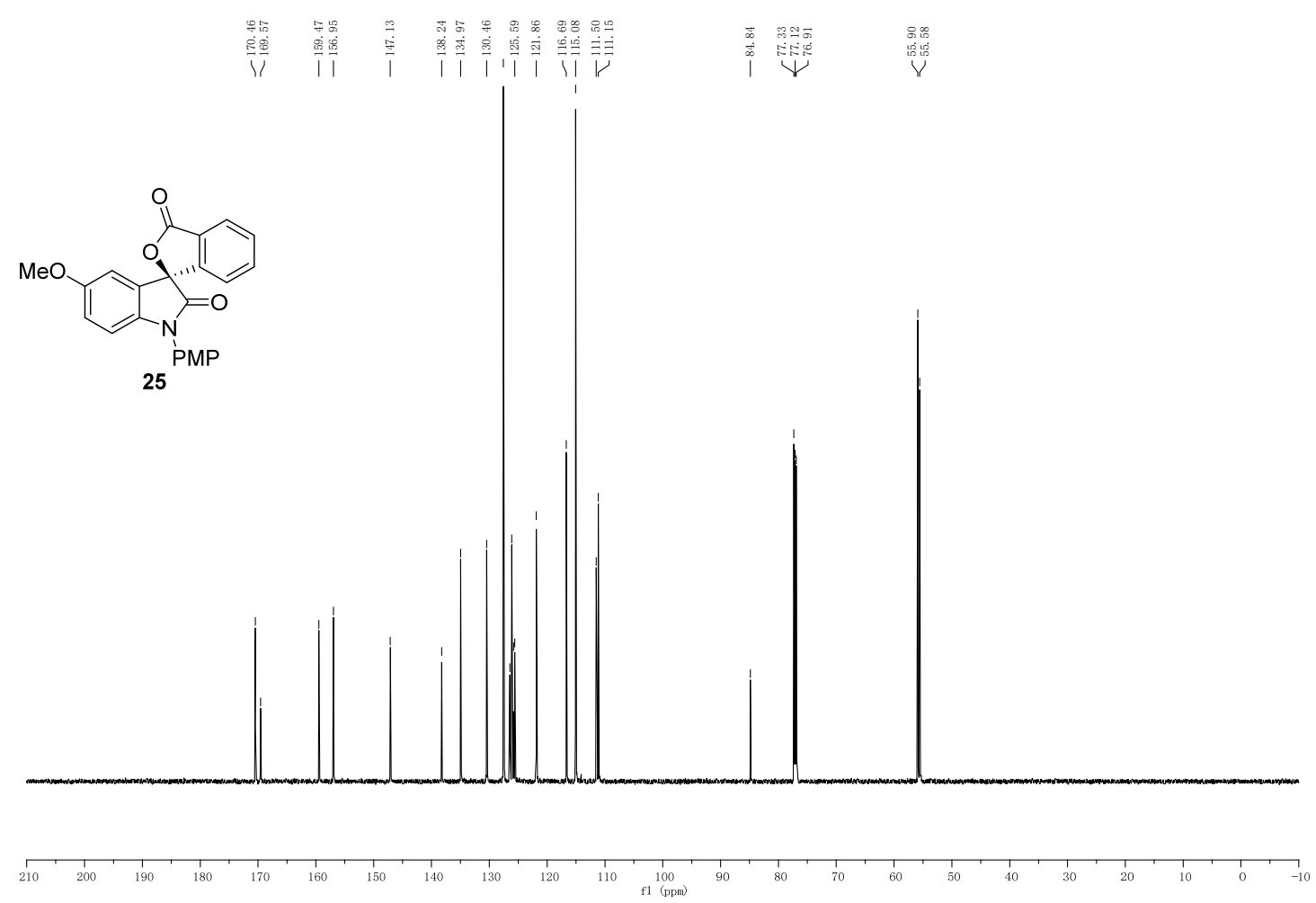
${ }^{1} \mathrm{H}$ NMR (600 MHz, $\mathrm{CDCl}_{3}$ ) of $\mathbf{2 6}$

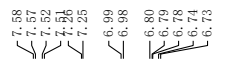

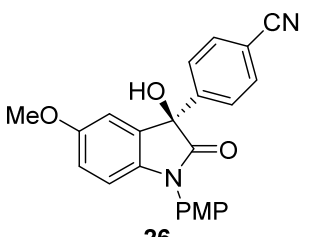

26

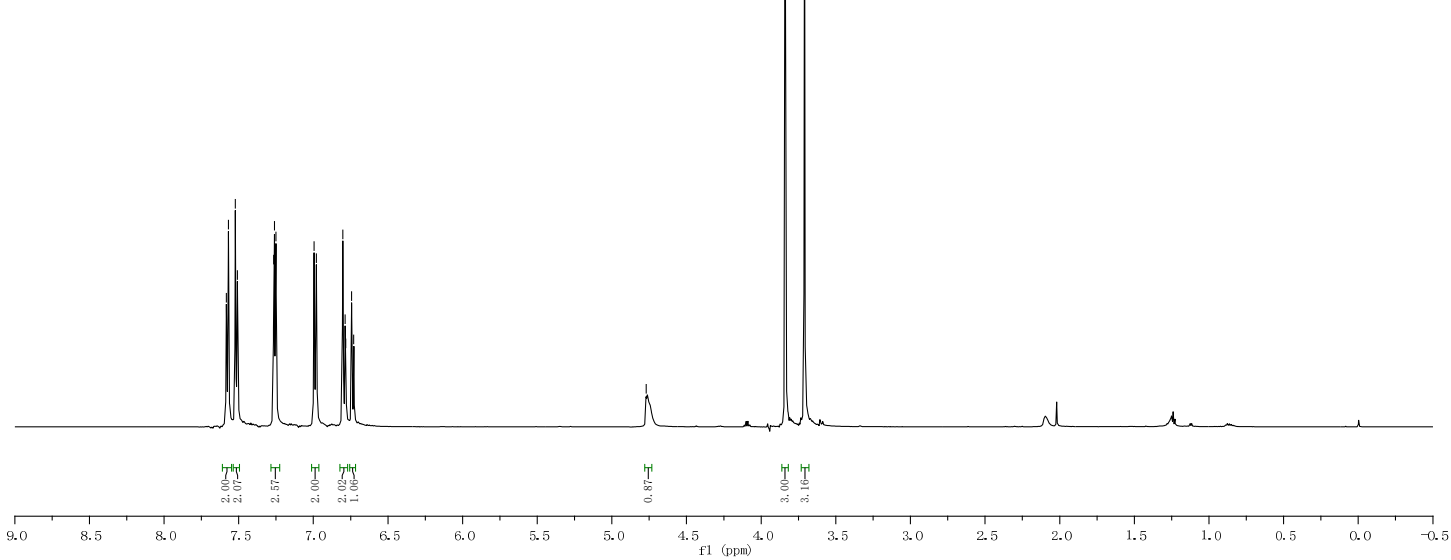

${ }^{13} \mathrm{C}$ NMR $\left(150 \mathrm{MHz}, \mathrm{CDCl}_{3}\right)$ of 26

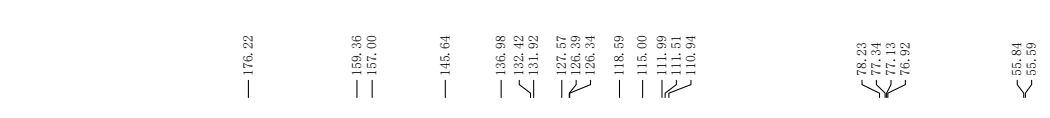
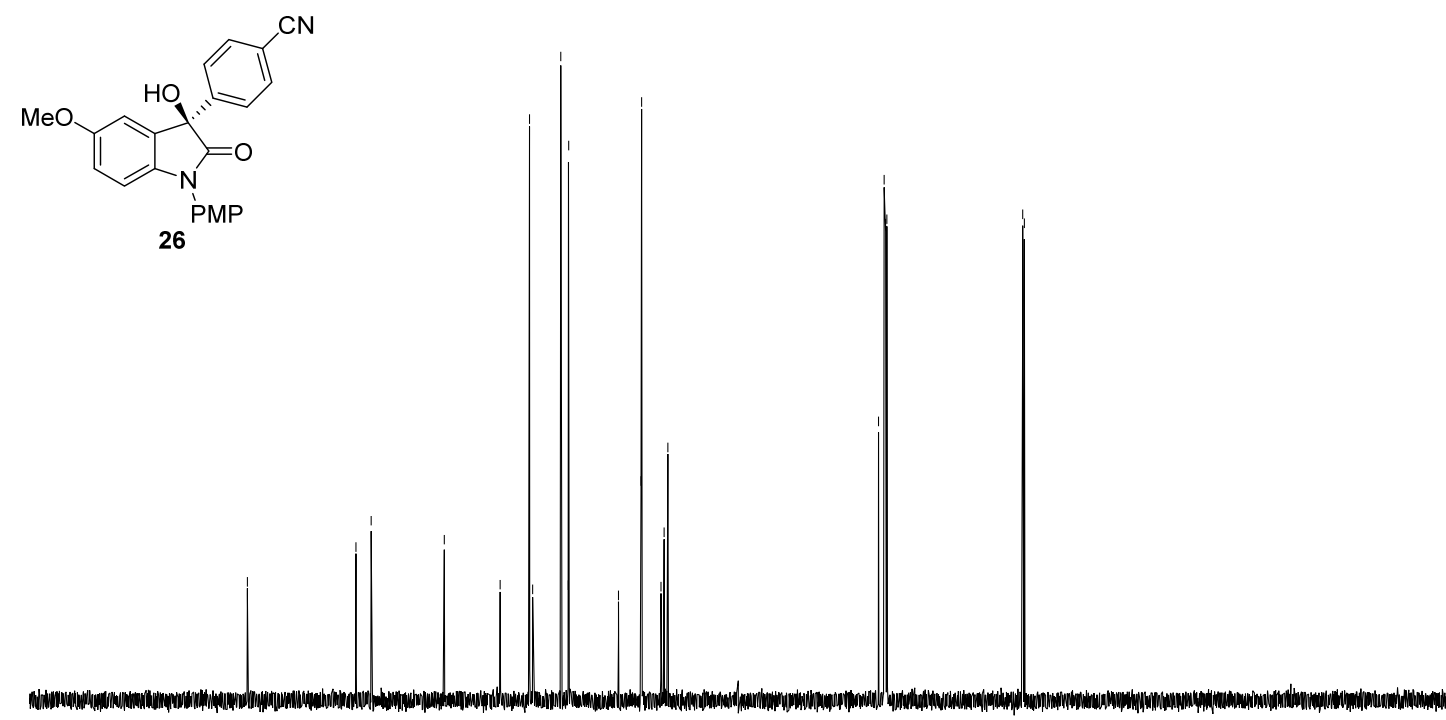

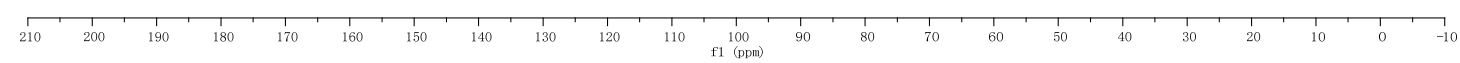


${ }^{1} \mathrm{H}$ NMR (600 MHz, $\mathrm{CDCl}_{3}$ ) of 27

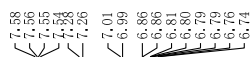

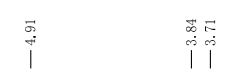

(1)

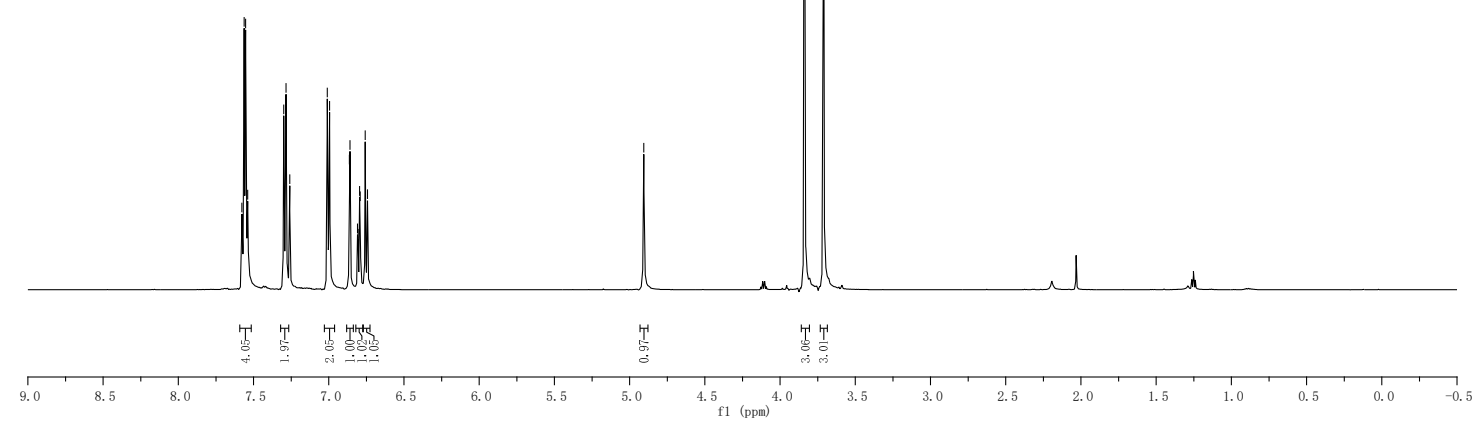

${ }^{13} \mathrm{C}$ NMR $\left(150 \mathrm{MHz}, \mathrm{CDCl}_{3}\right)$ of 27

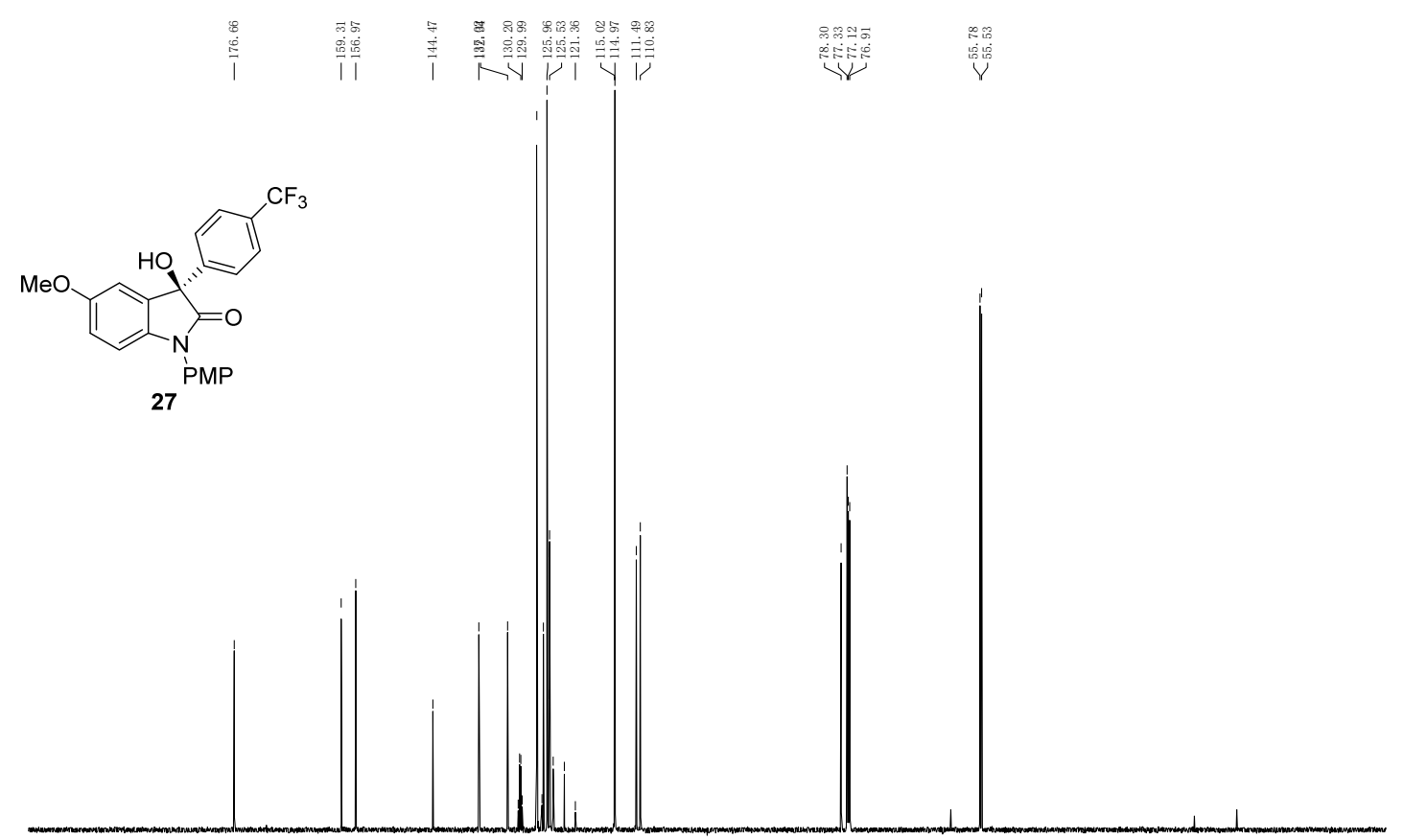

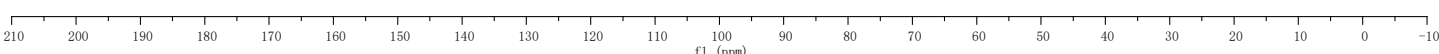


${ }^{19}$ F NMR (565 MHz, $\left.\mathrm{CDCl}_{3}\right)$ of 27

(1)

0
$\substack{2 \\ 0}$
1

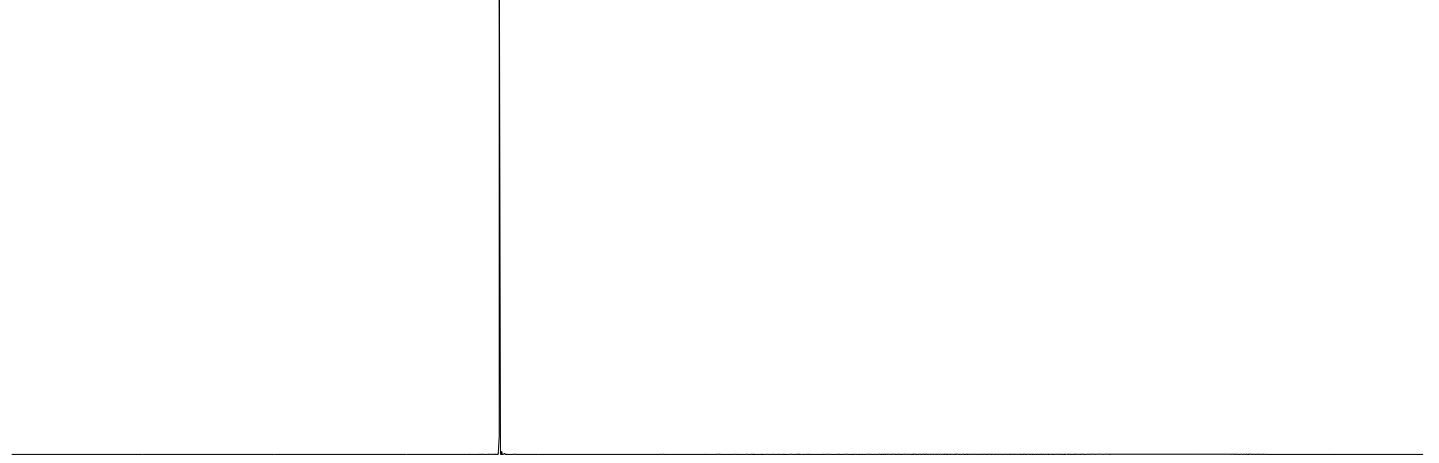

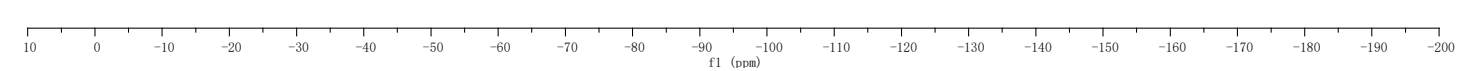


${ }^{1} \mathrm{H}$ NMR (600 MHz, $\mathrm{CDCl}_{3}$ ) of $\mathbf{2 8}$

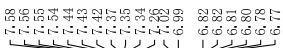
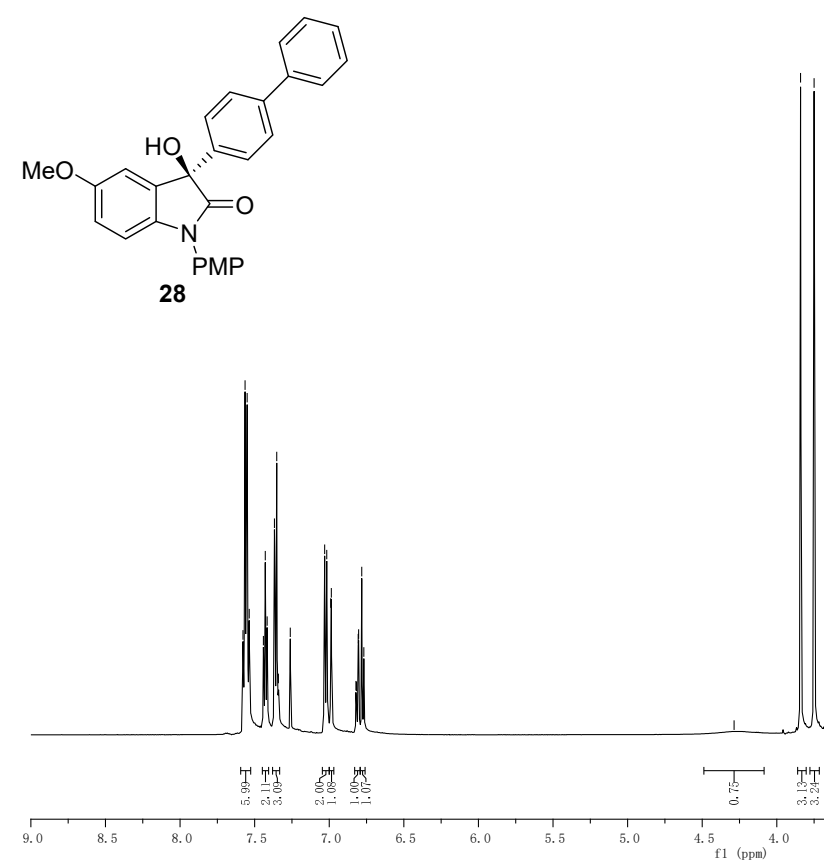

${ }^{13} \mathrm{C}$ NMR $\left(150 \mathrm{MHz}, \mathrm{CDCl}_{3}\right)$ of $\mathbf{2 8}$
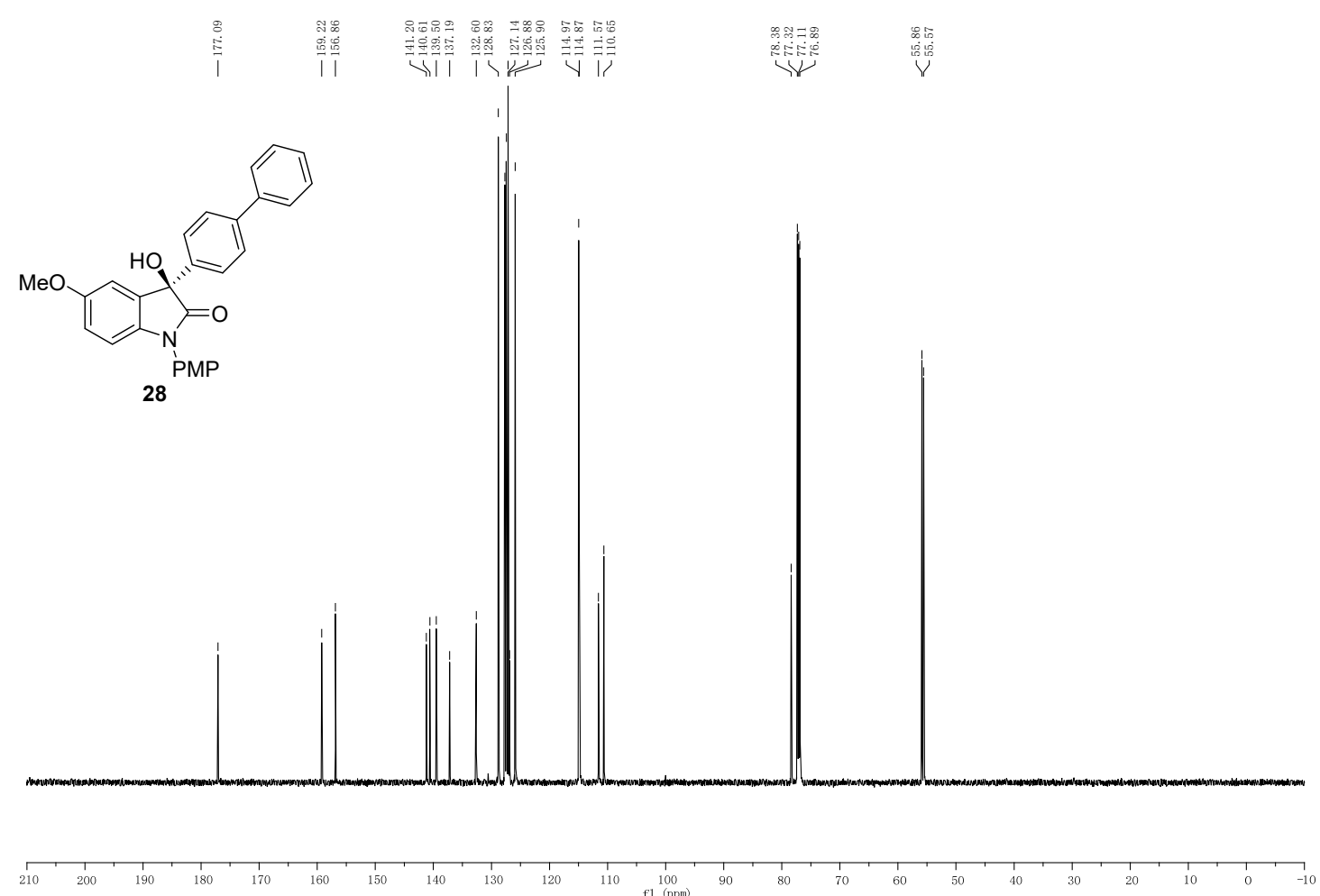
${ }^{1} \mathrm{H}$ NMR (600 MHz, $\mathrm{CDCl}_{3}$ ) of 29

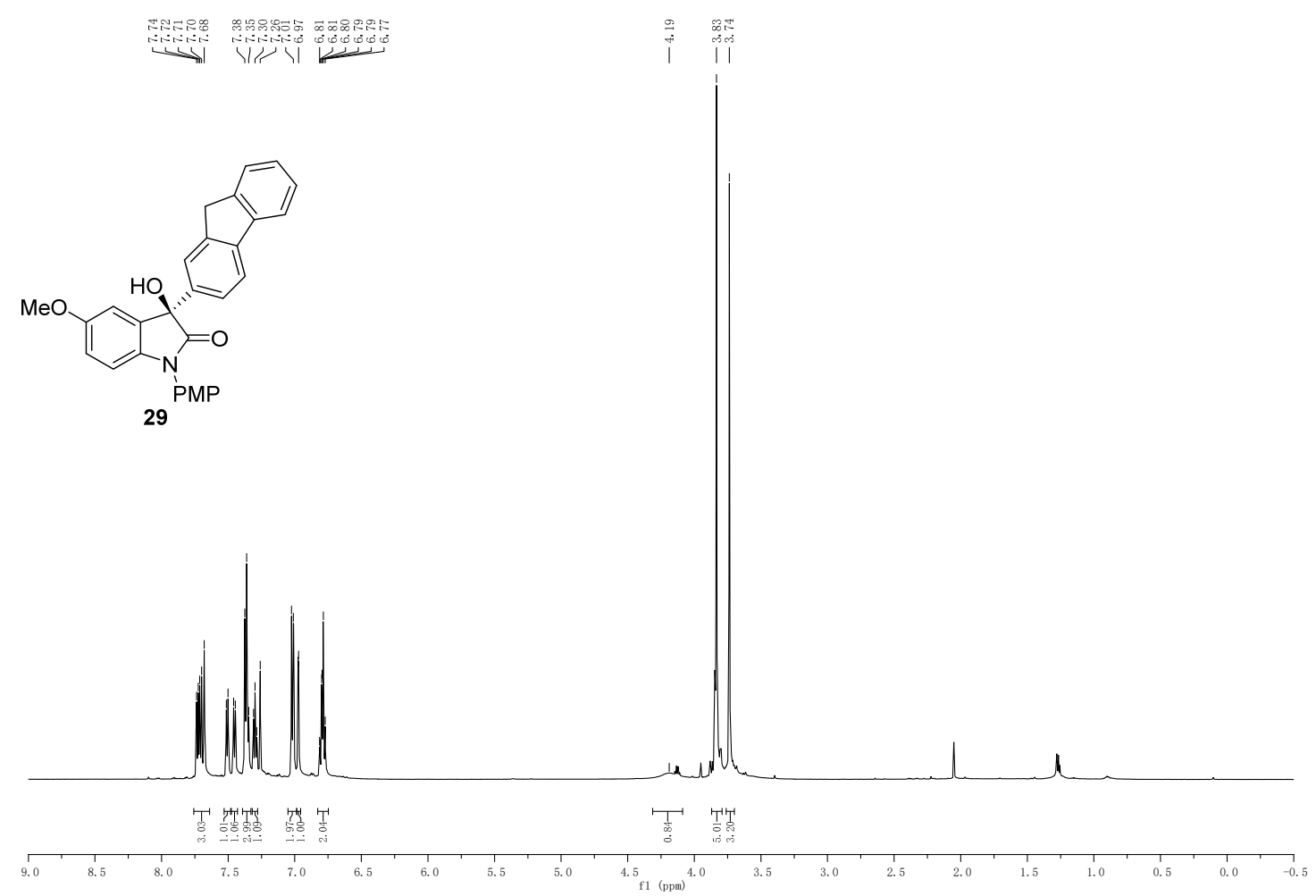

${ }^{13} \mathrm{C}$ NMR (150 MHz, $\mathrm{CDCl}_{3}$ ) of 29

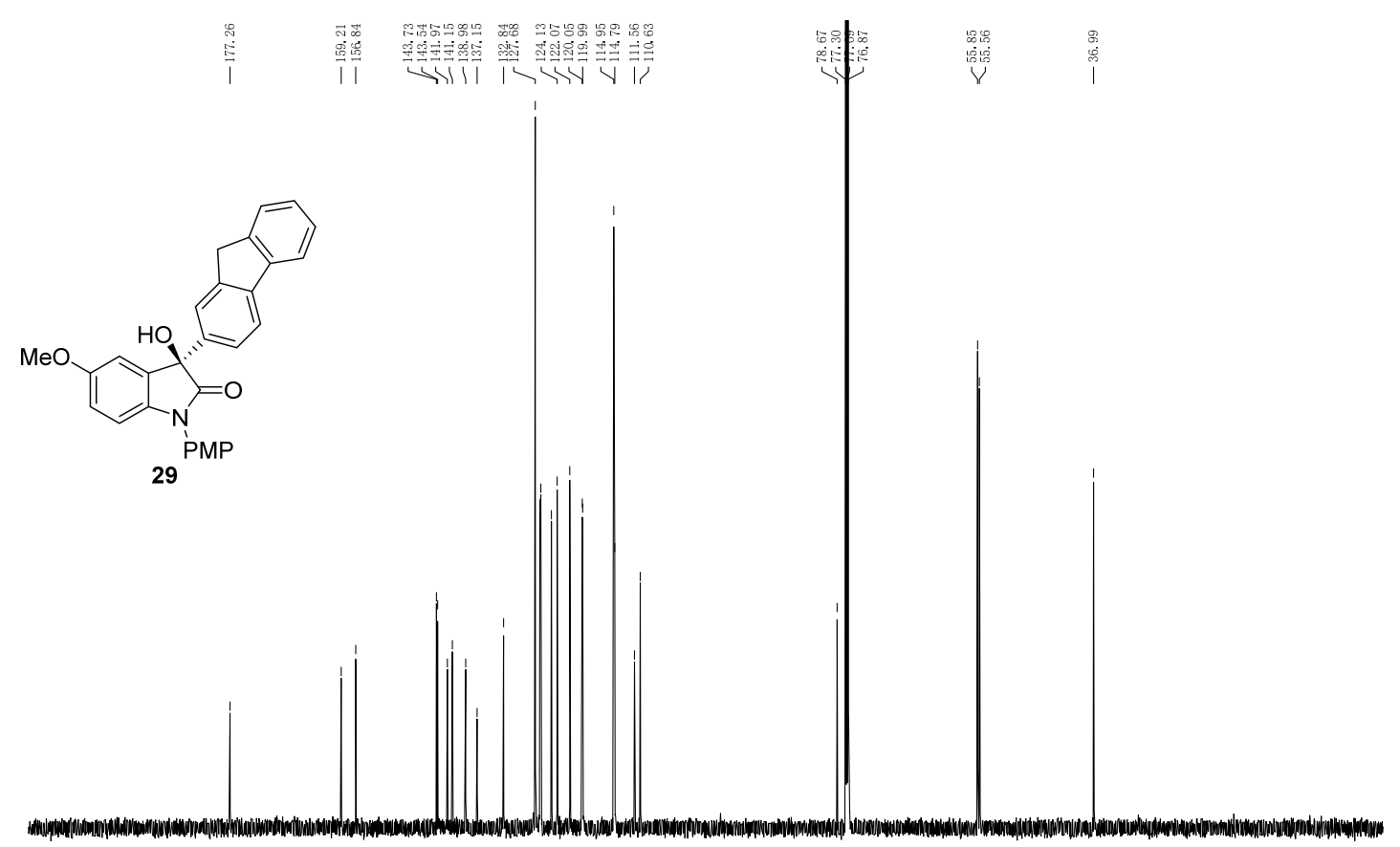

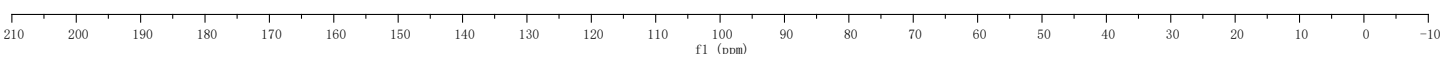


${ }^{1} \mathrm{H}$ NMR (600 MHz, $\mathrm{CDCl}_{3}$ ) of $\mathbf{3 0}$



${ }^{13} \mathrm{C}$ NMR $\left(150 \mathrm{MHz}, \mathrm{CDCl}_{3}\right)$ of 30
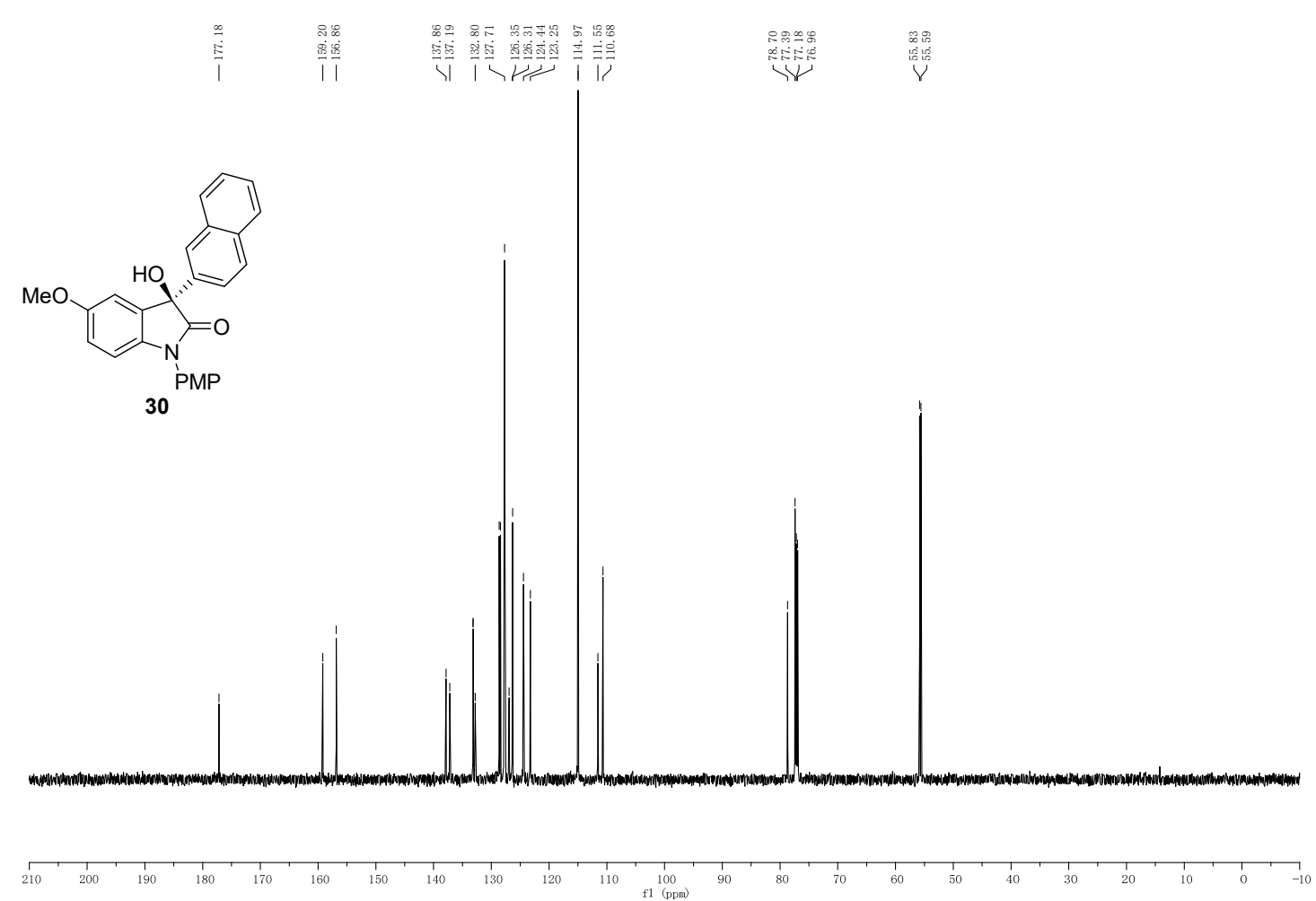
${ }^{1} \mathrm{H}$ NMR (600 MHz, $\mathrm{CDCl}_{3}$ ) of $\mathbf{3 1}$

践

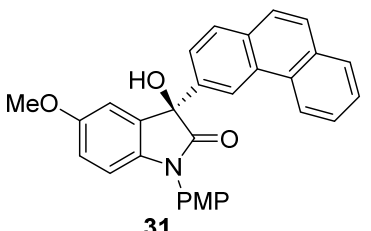

31

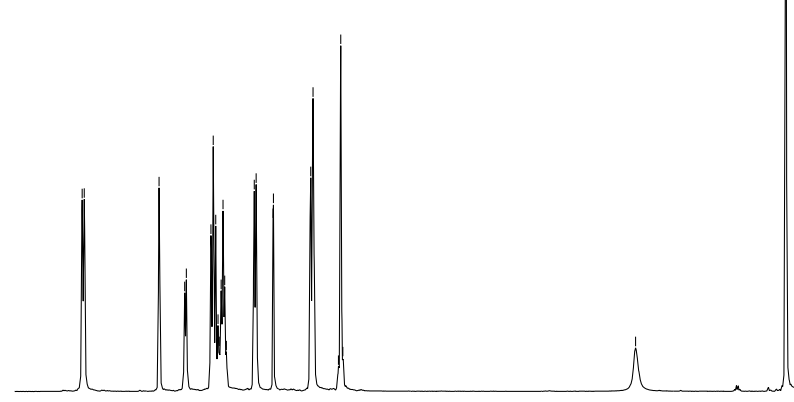

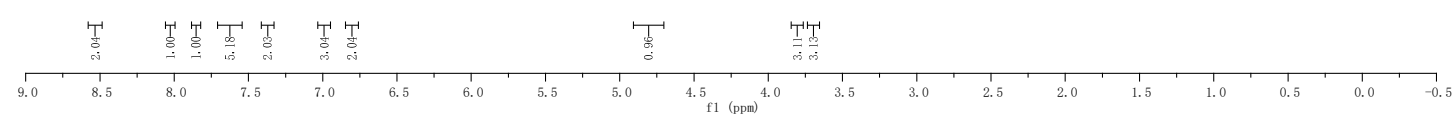

${ }^{13} \mathrm{C}$ NMR $\left(150 \mathrm{MHz}, \mathrm{CDCl}_{3}\right)$ of $\mathbf{3 1}$

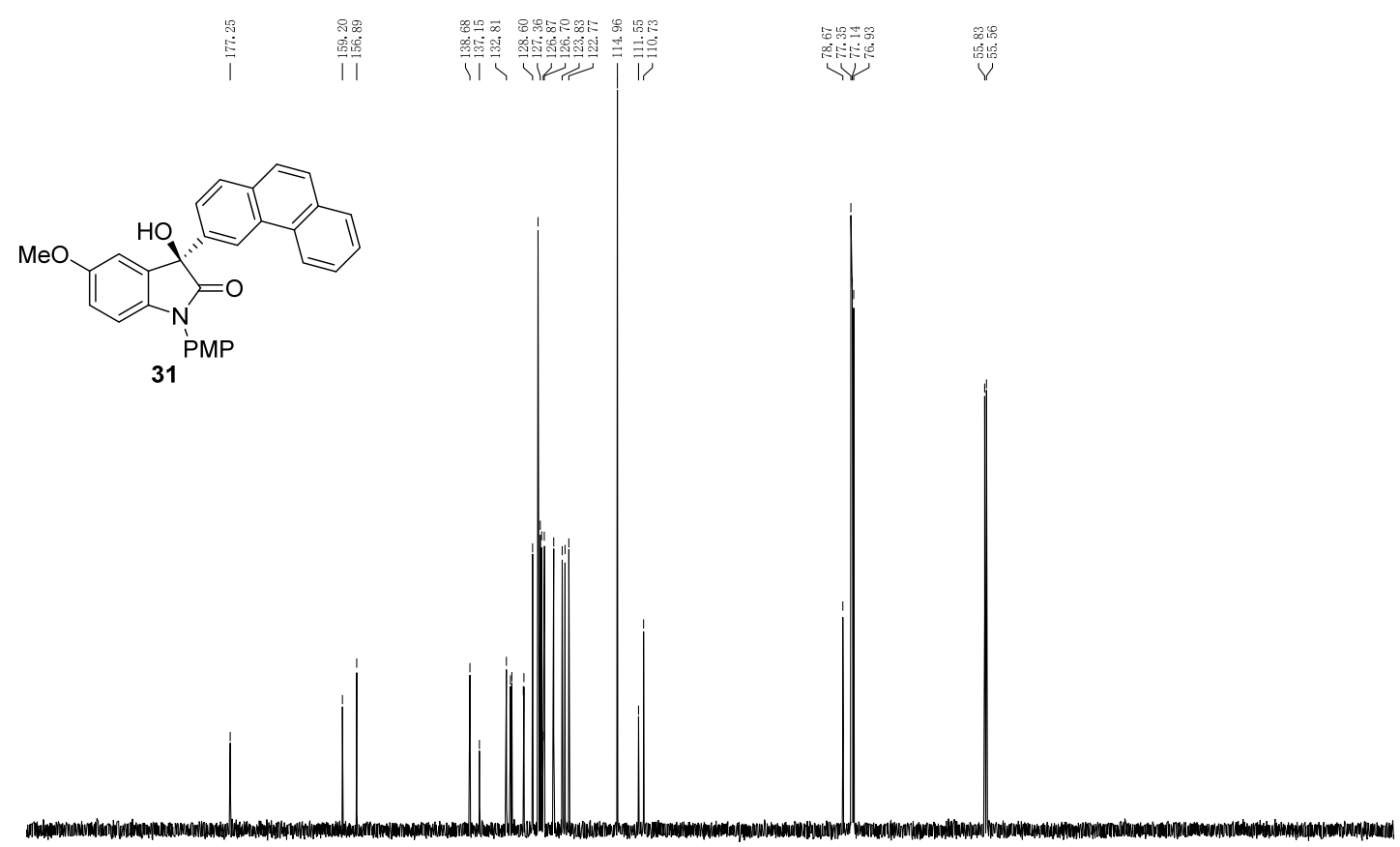

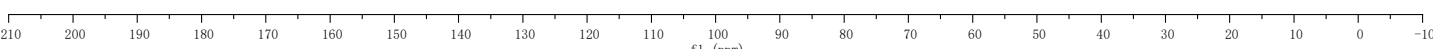


${ }^{1} \mathrm{H}$ NMR (600 MHz, $\mathrm{CDCl}_{3}$ ) of $\mathbf{3 2}$

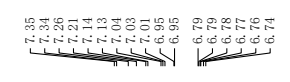

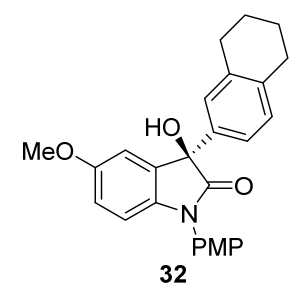

32
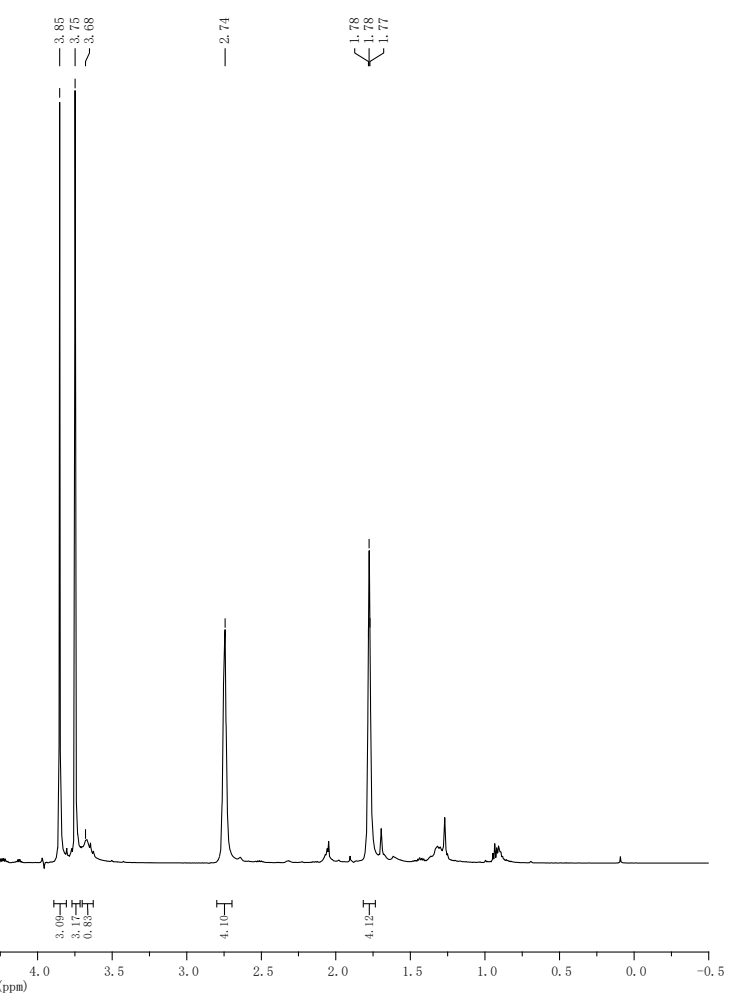

${ }^{13} \mathrm{C} \mathrm{NMR}\left(150 \mathrm{MHz}, \mathrm{CDCl}_{3}\right)$ of 32
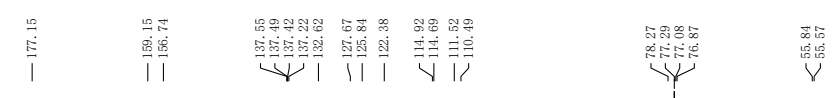

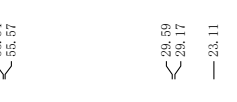

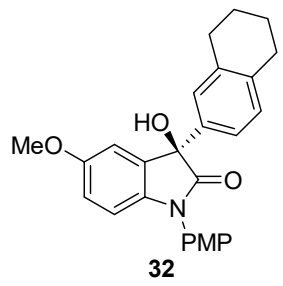

32

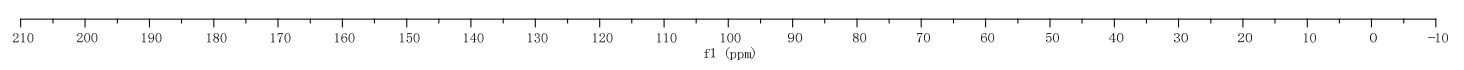

S80 
${ }^{1} \mathrm{H}$ NMR (600 MHz, $\mathrm{CDCl}_{3}$ ) of $\mathbf{3 3}$

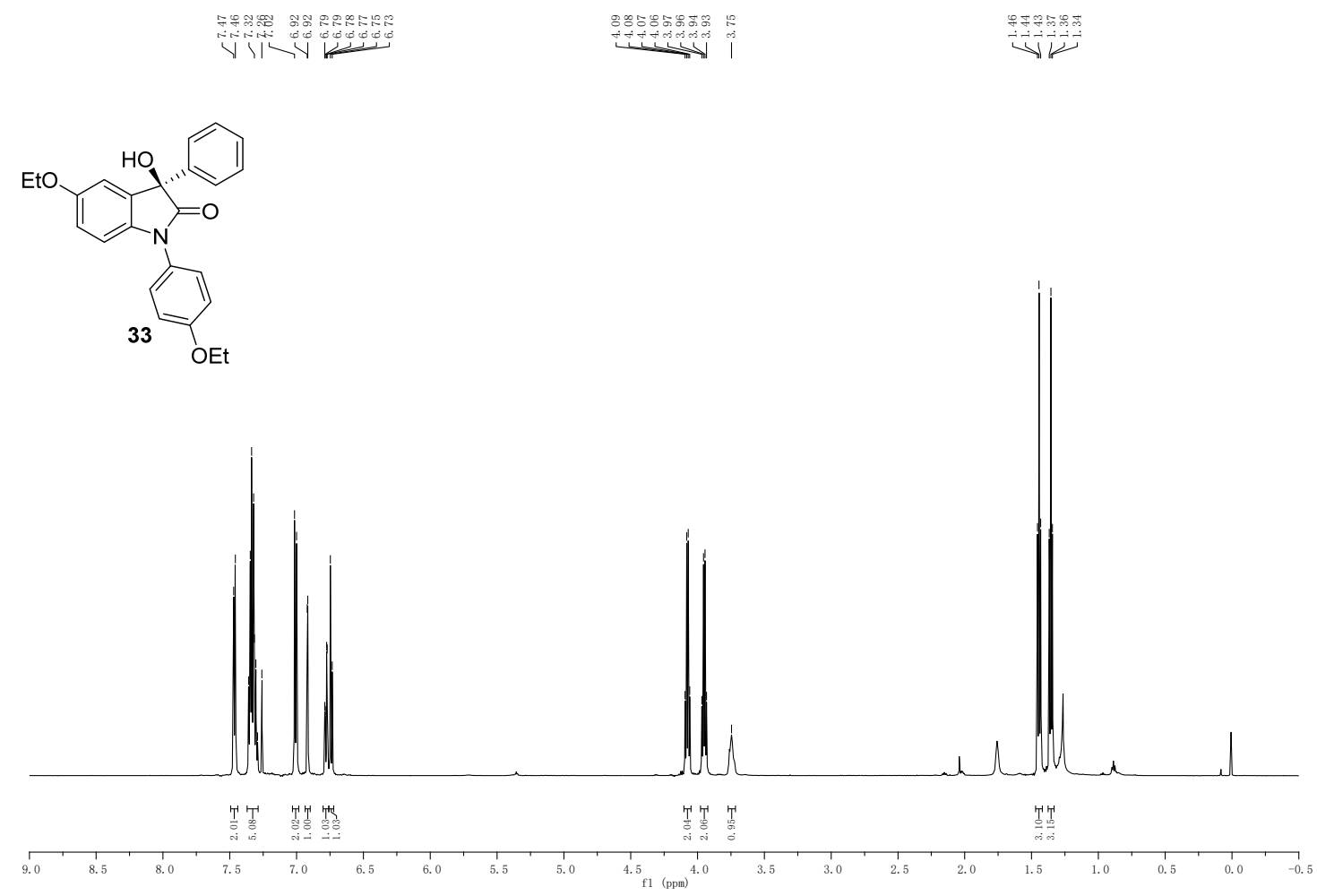

${ }^{13} \mathrm{C}$ NMR $\left(150 \mathrm{MHz}, \mathrm{CDCl}_{3}\right)$ of 33

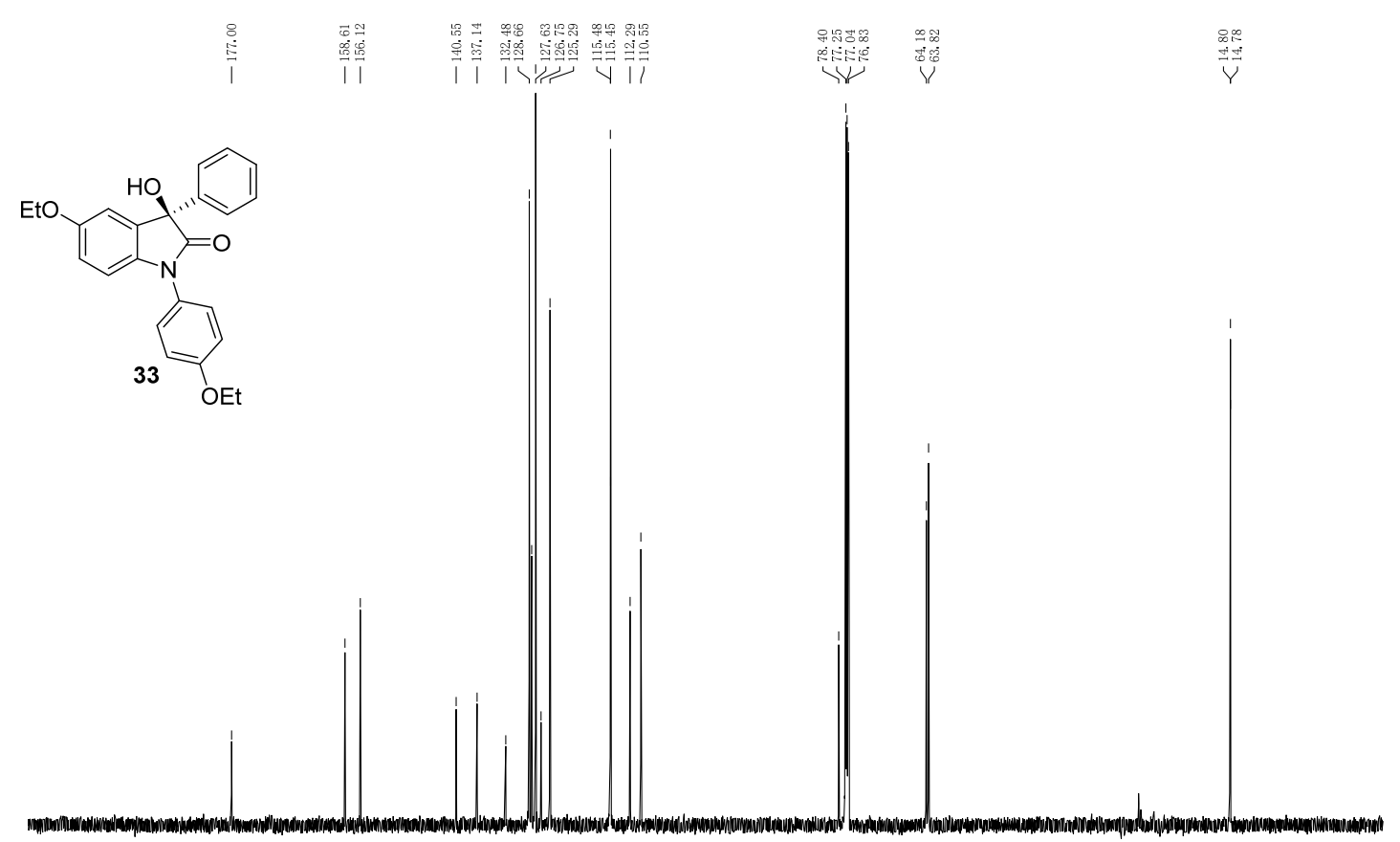

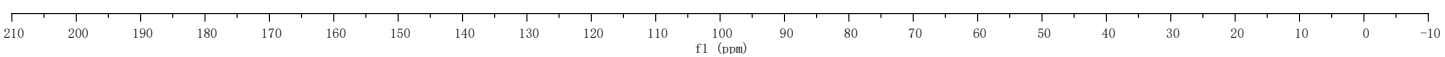


${ }^{1} \mathrm{H}$ NMR (600 MHz, $\mathrm{CDCl}_{3}$ ) of $\mathbf{3 4}$

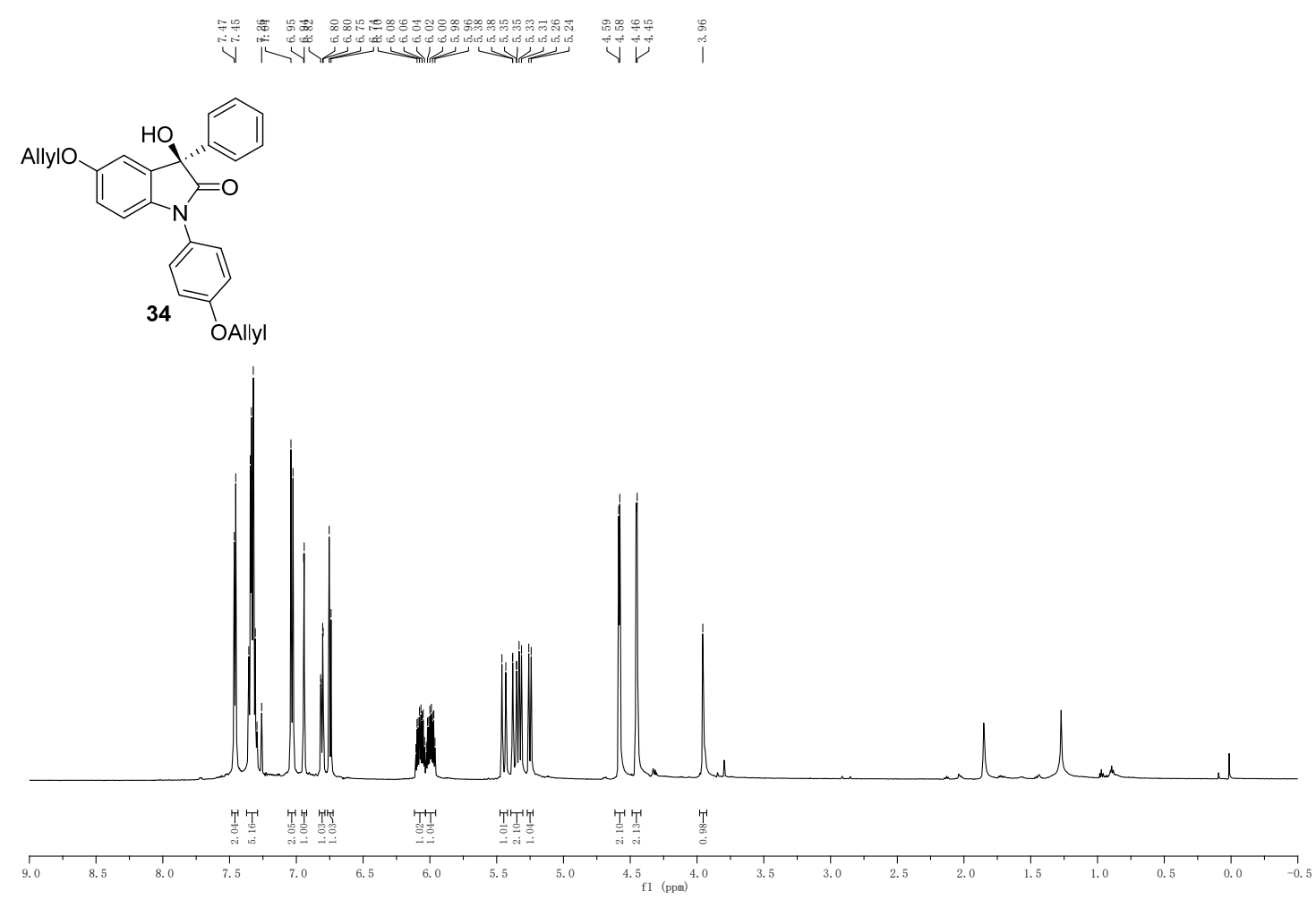

${ }^{13} \mathrm{C}$ NMR $\left(150 \mathrm{MHz}, \mathrm{CDCl}_{3}\right)$ of 34
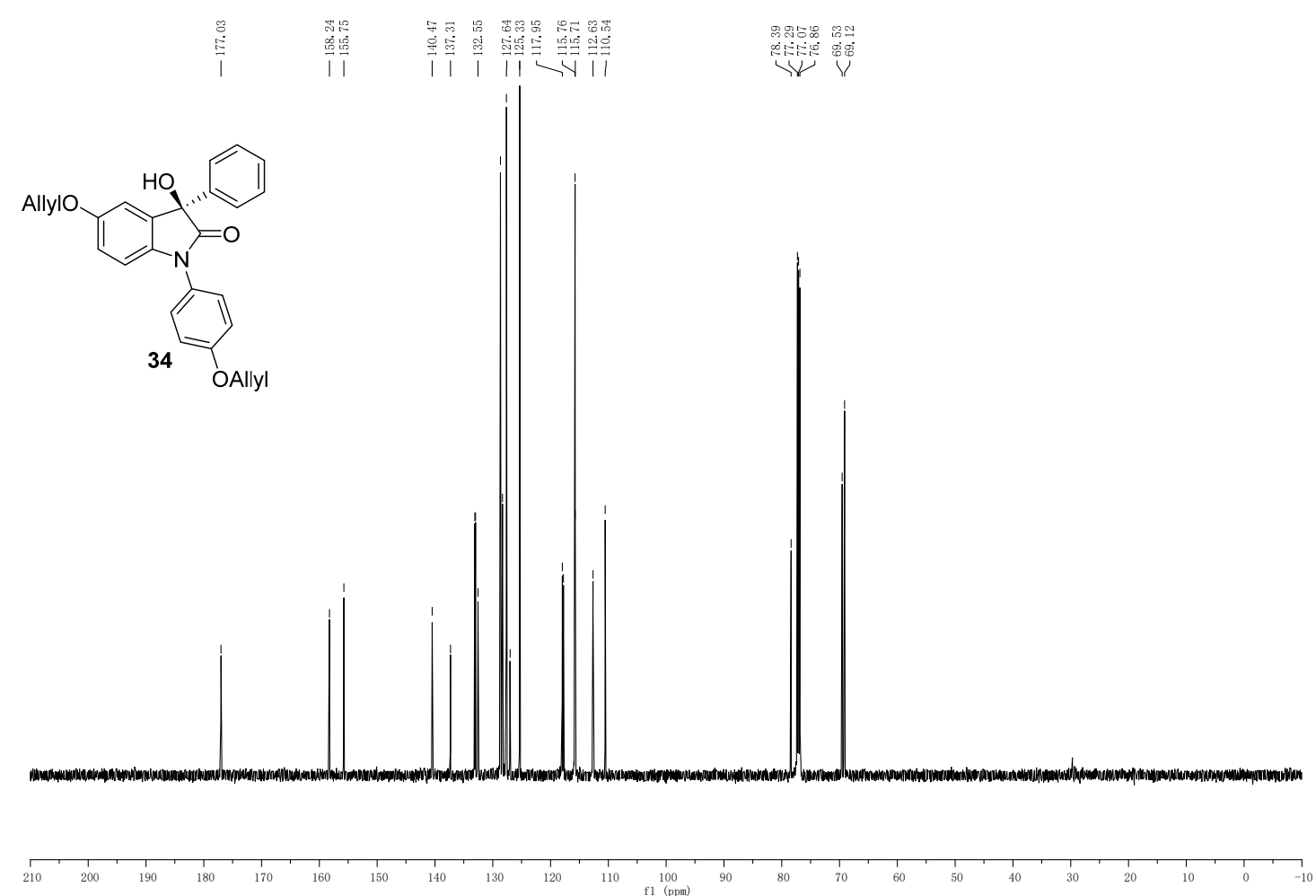
${ }^{1} \mathrm{H}$ NMR (600 MHz, $\mathrm{CDCl}_{3}$ ) of $\mathbf{3 5}$

|
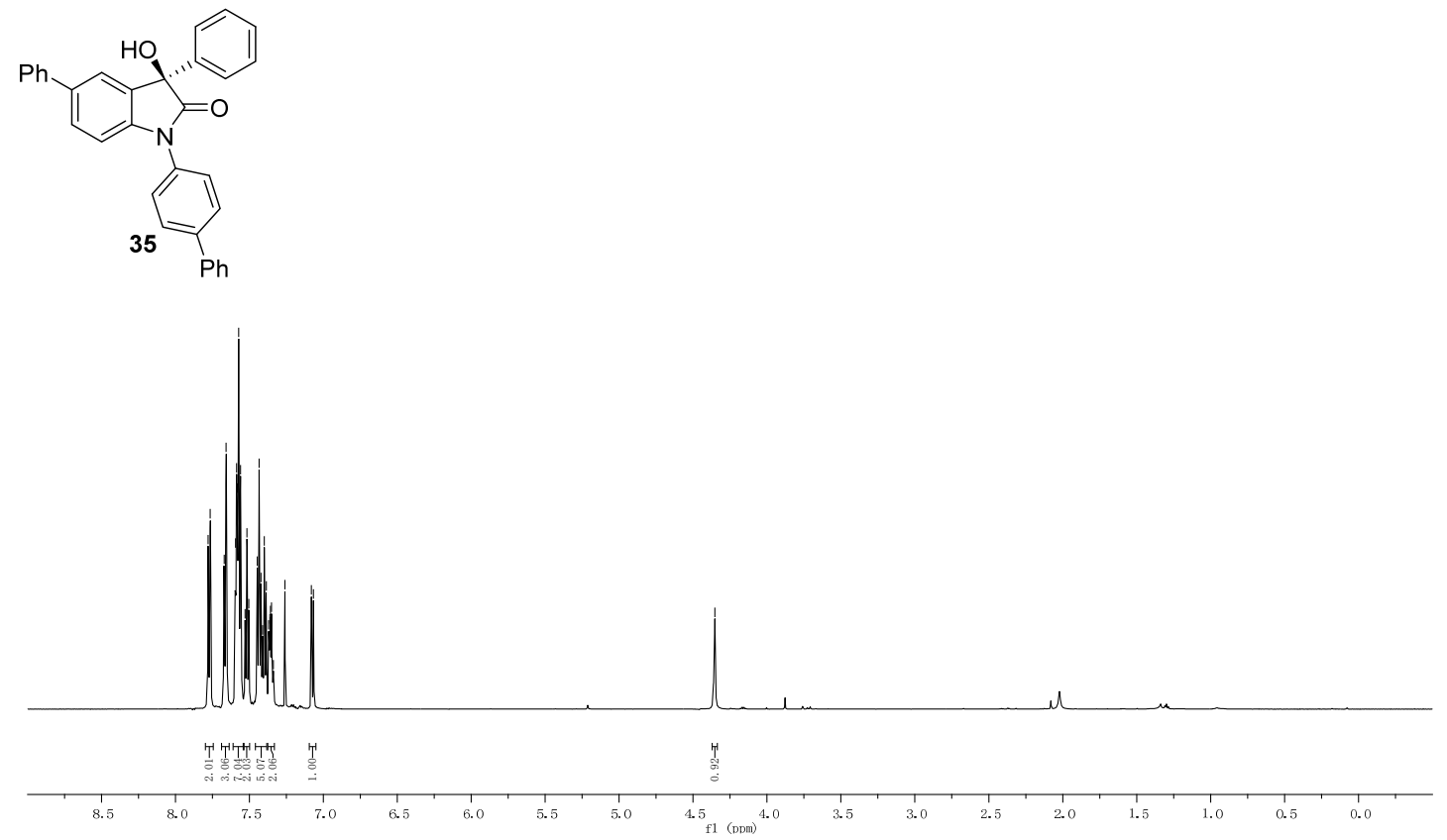

${ }^{13} \mathrm{C}$ NMR $\left(150 \mathrm{MHz}, \mathrm{CDCl}_{3}\right)$ of 35

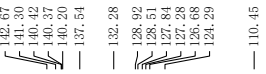

$\underbrace{4}$
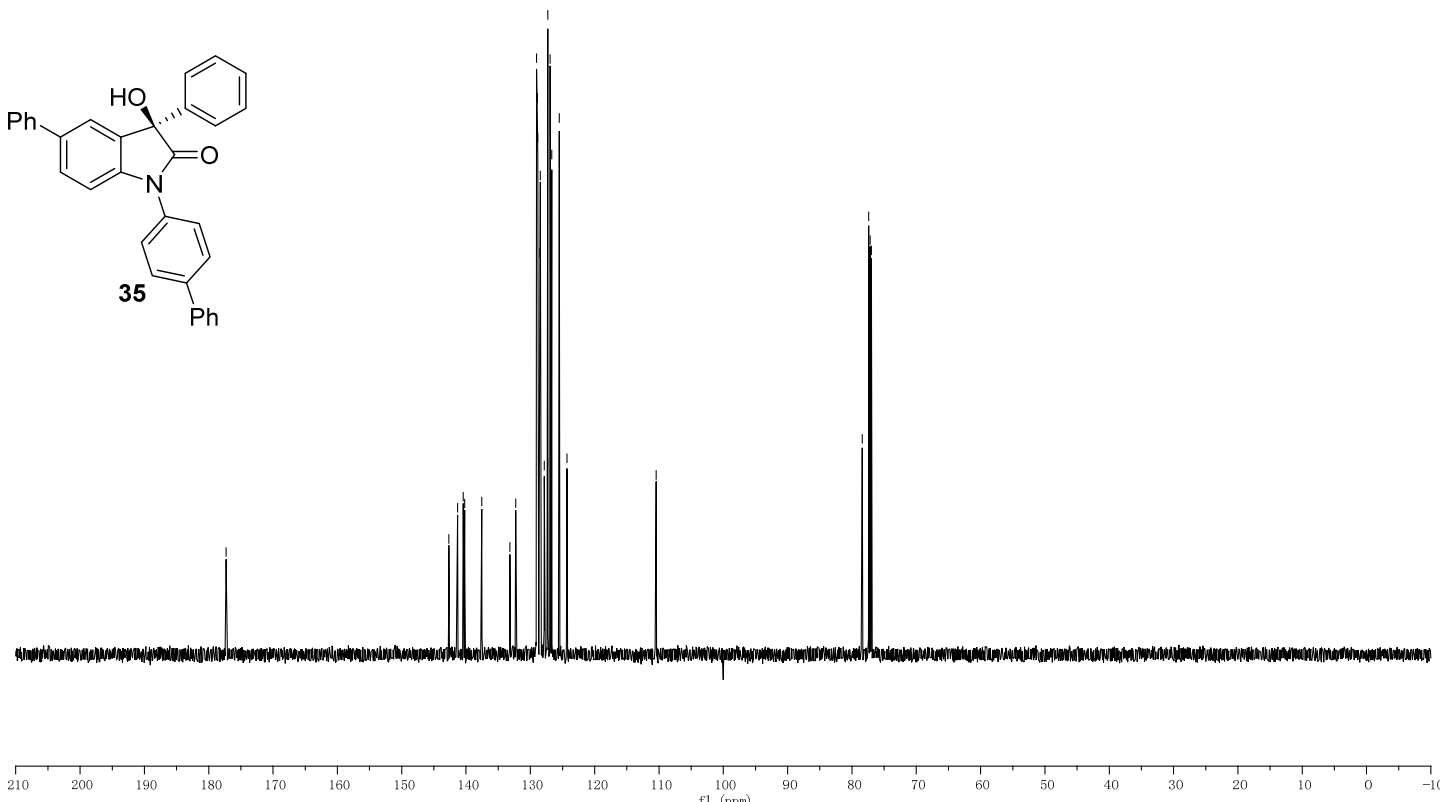
${ }^{1} \mathrm{H} \mathrm{NMR}\left(600 \mathrm{MHz}, \mathrm{CDCl}_{3}\right)$ of $\mathbf{3 6}$

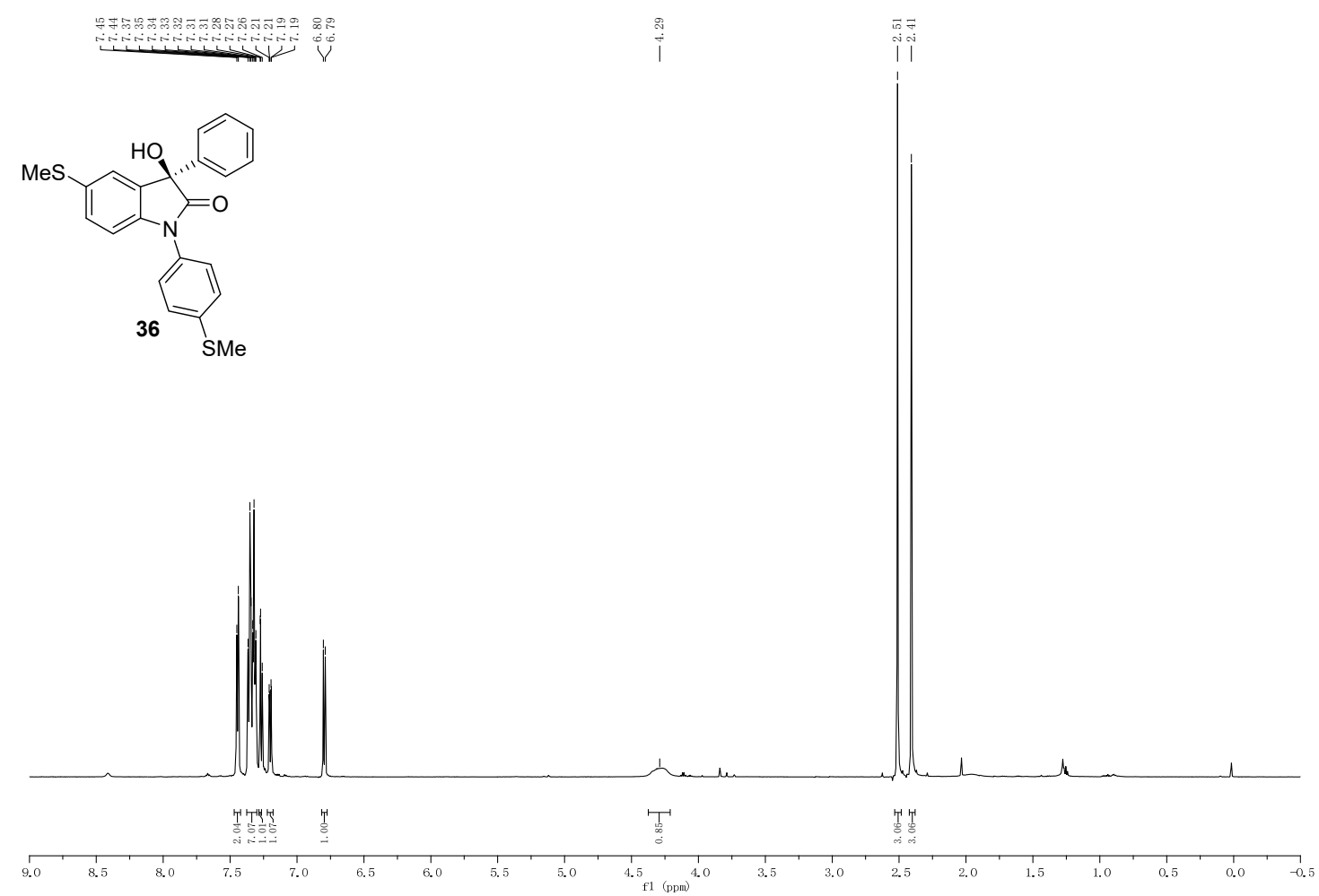

${ }^{13} \mathrm{C}$ NMR $\left(150 \mathrm{MHz}, \mathrm{CDCl}_{3}\right)$ of 36
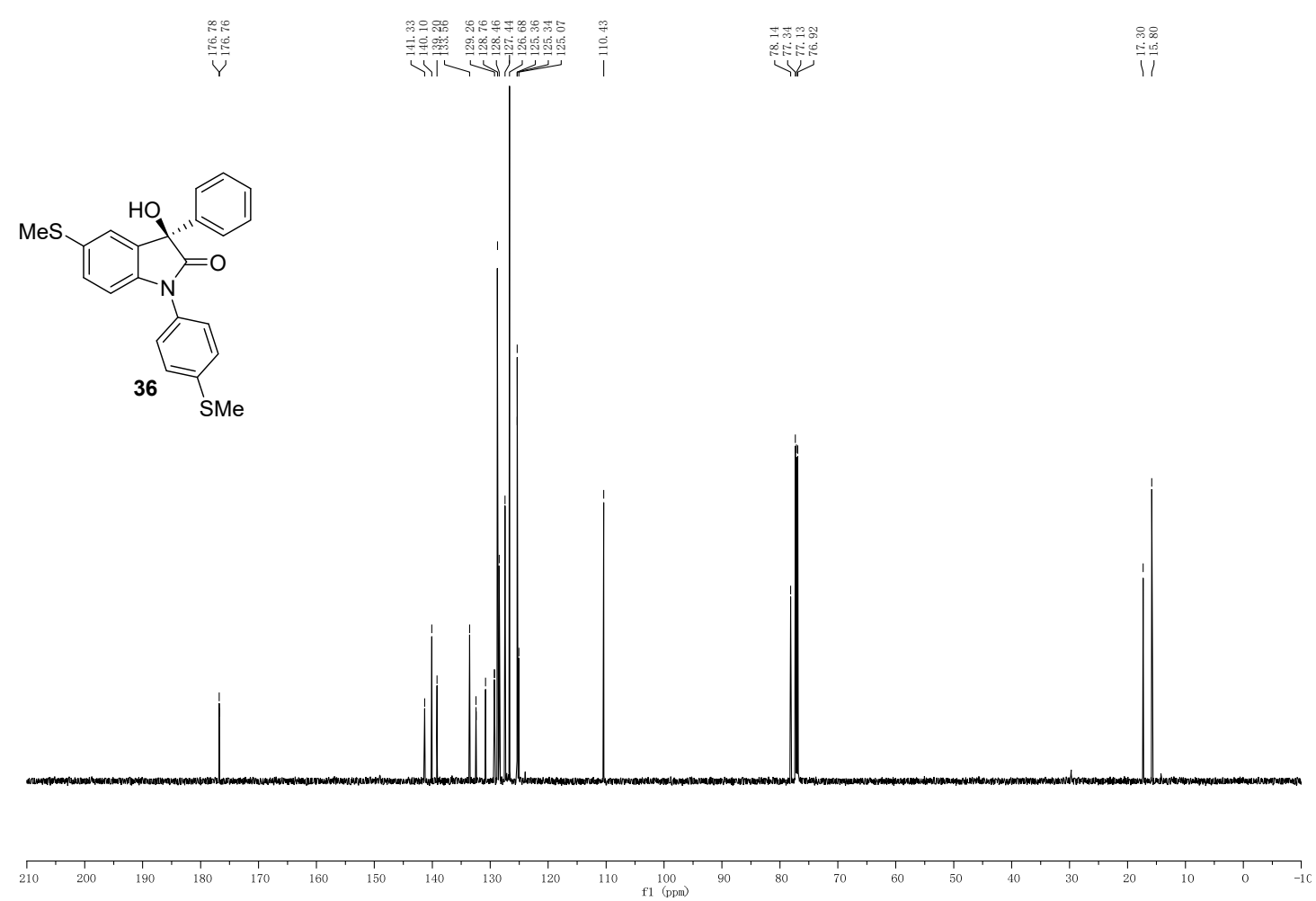
${ }^{1} \mathrm{H}$ NMR (600 MHz, $\mathrm{CDCl}_{3}$ ) of $\mathbf{3 7}$

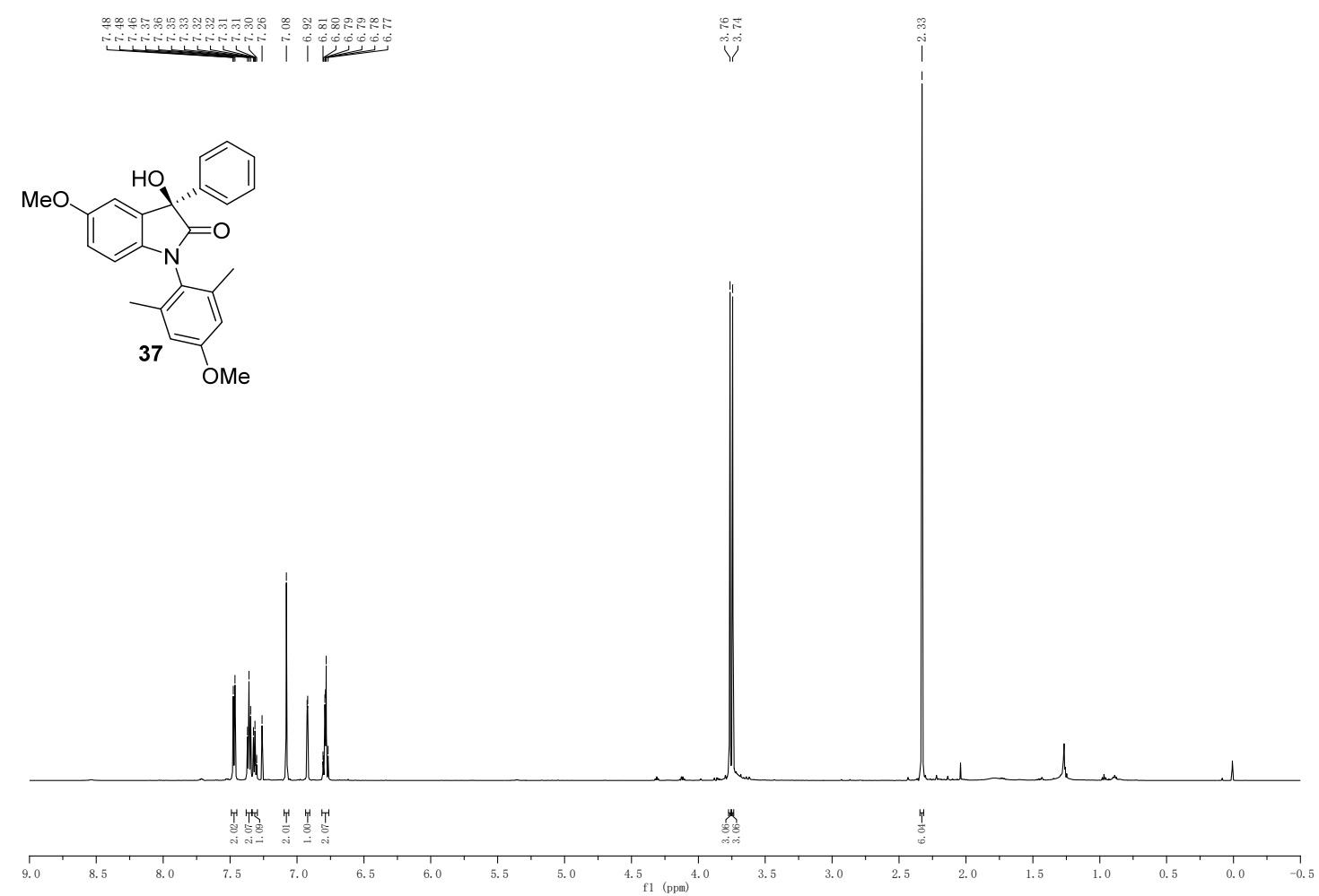

${ }^{13} \mathrm{C}$ NMR $\left(150 \mathrm{MHz}, \mathrm{CDCl}_{3}\right)$ of 37

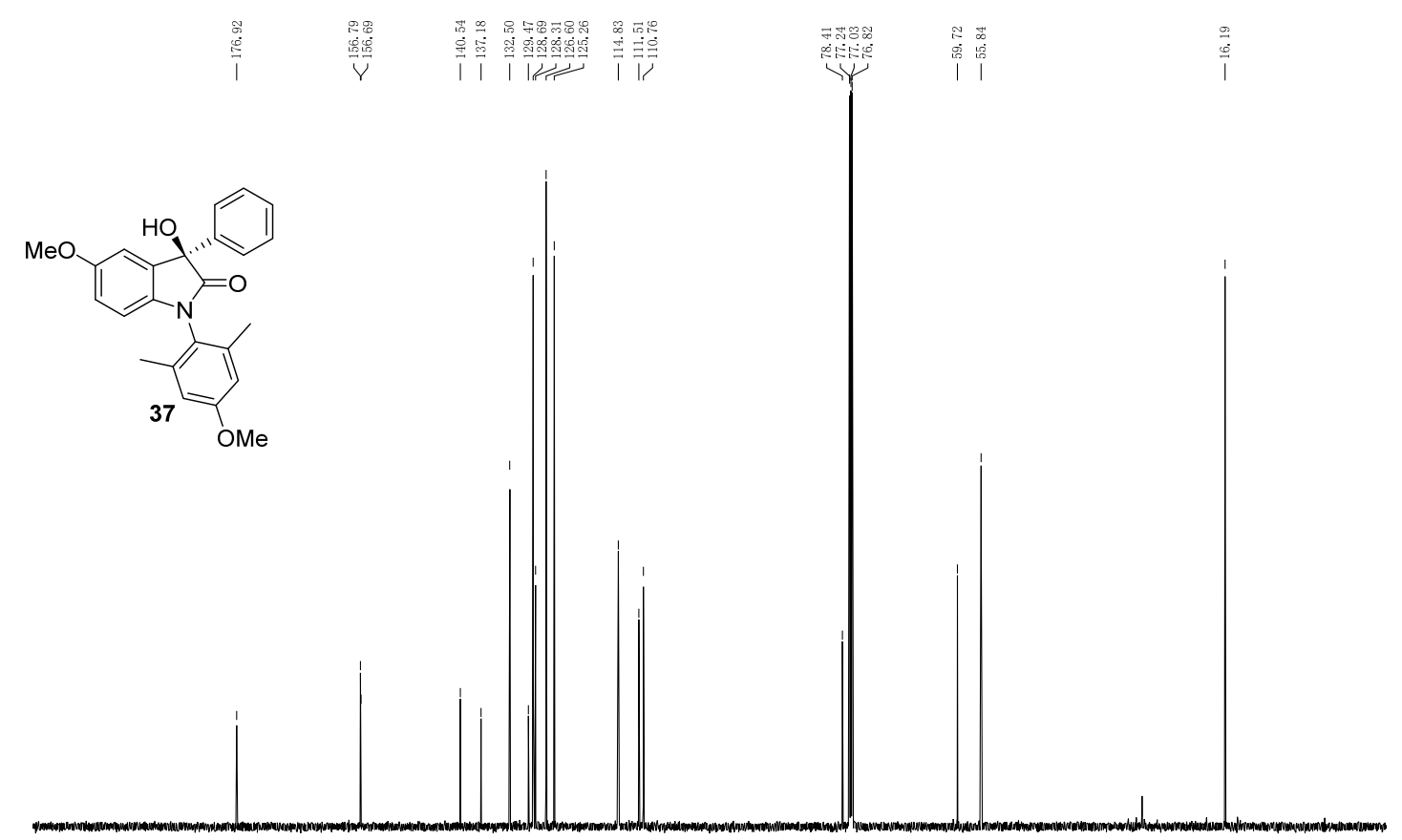


${ }^{1} \mathrm{H}$ NMR (600 MHz, $\left.\mathrm{CDCl}_{3}\right)$ of $\mathbf{3 8}$

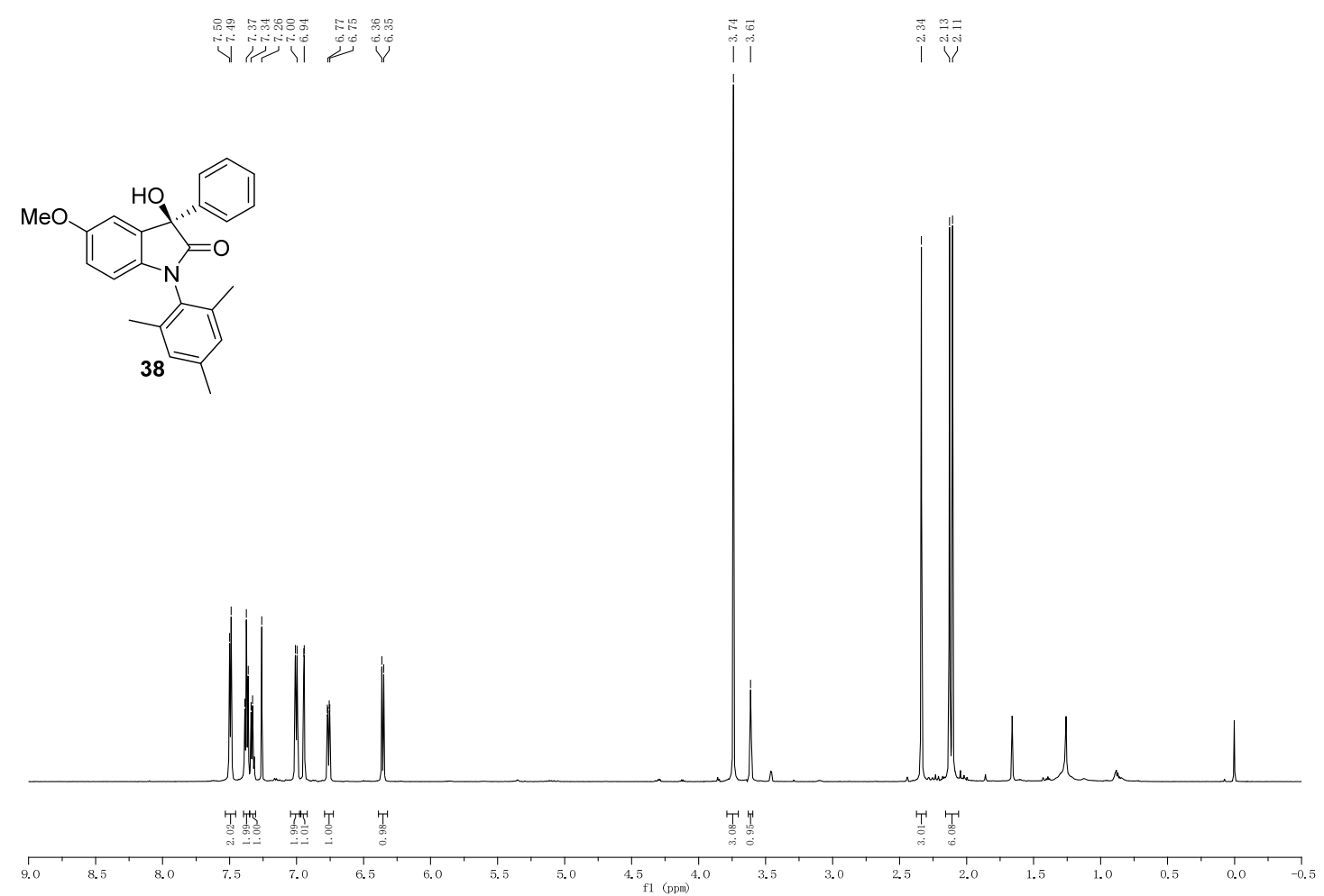

${ }^{13} \mathrm{C}$ NMR $\left(150 \mathrm{MHz}, \mathrm{CDCl}_{3}\right)$ of $\mathbf{3 8}$
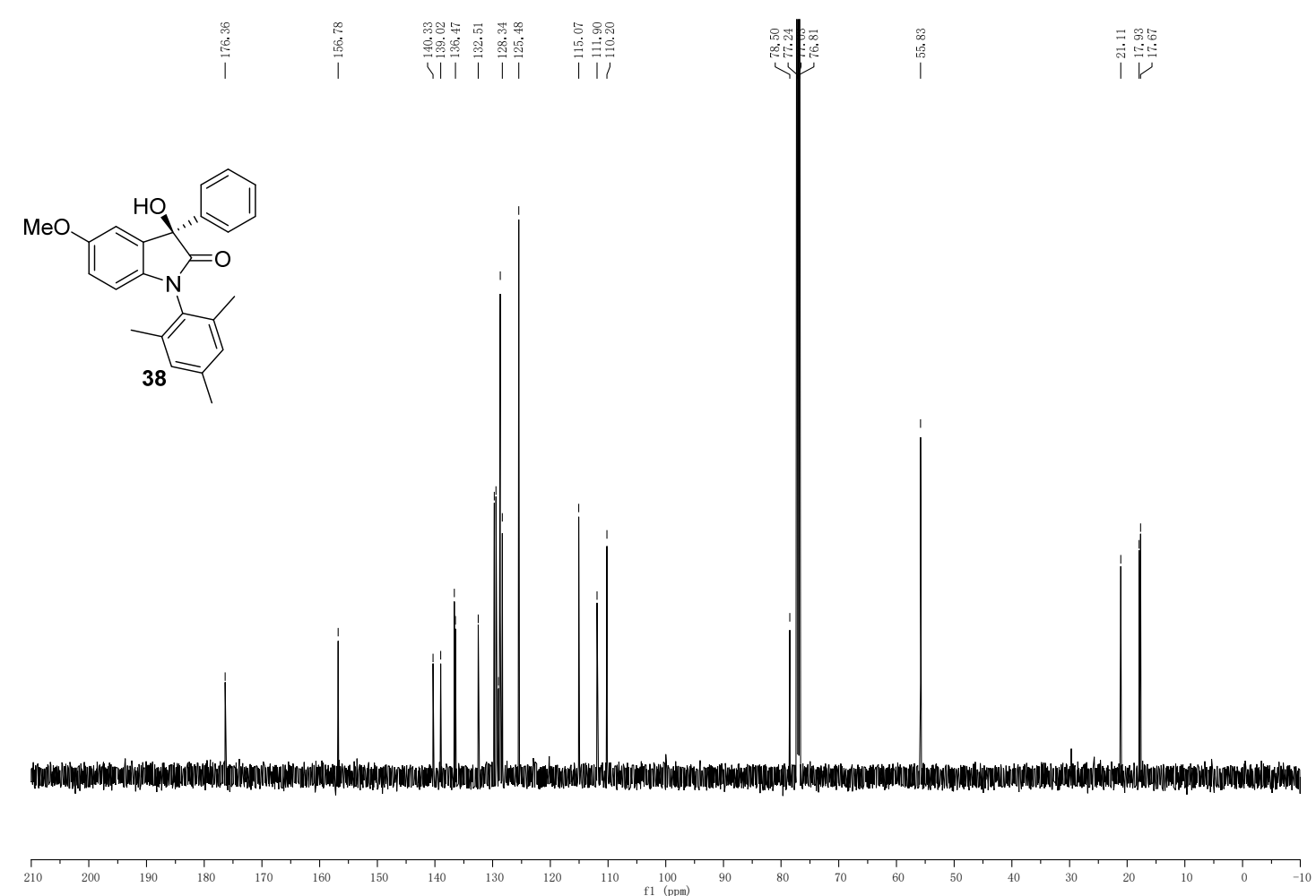
${ }^{1} \mathrm{H}$ NMR $\left(600 \mathrm{MHz}, \mathrm{CDCl}_{3}\right)$ of $\mathbf{4 1}$

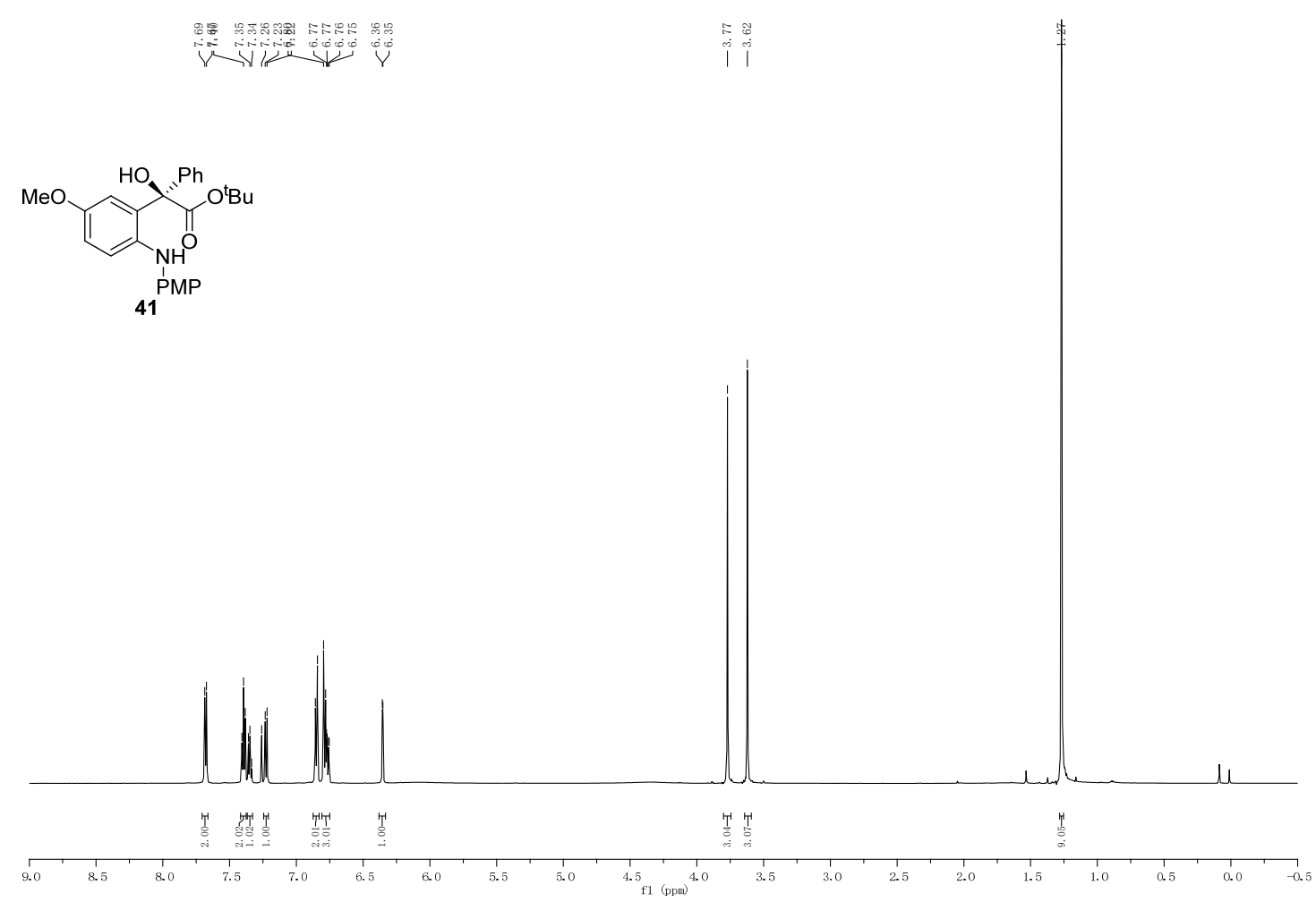

${ }^{13} \mathrm{C} \mathrm{NMR}\left(150 \mathrm{MHz}, \mathrm{CDCl}_{3}\right)$ of $4 \mathbf{1}$
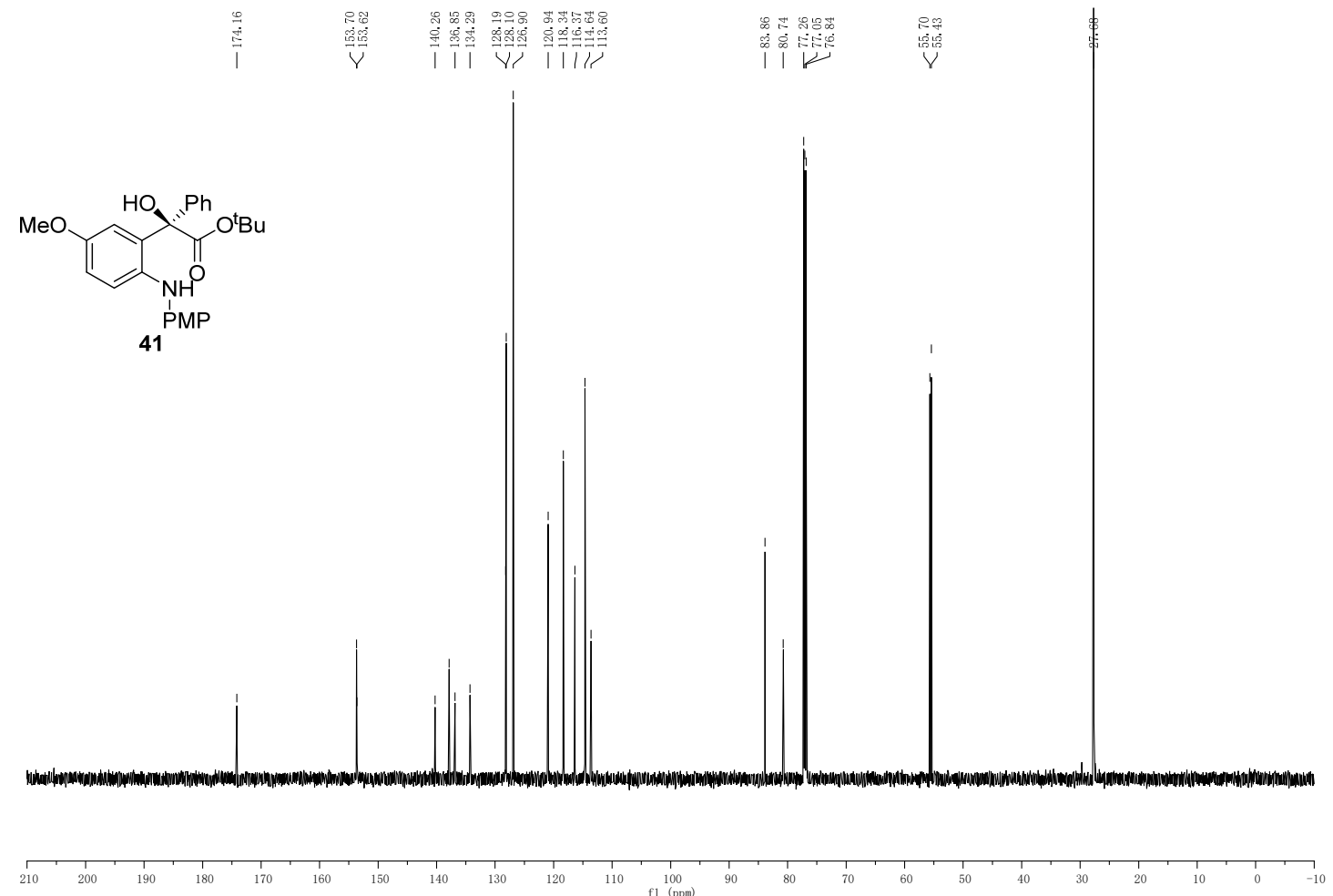


\section{HPLC Spectra}

HPLC Spectra of 3.
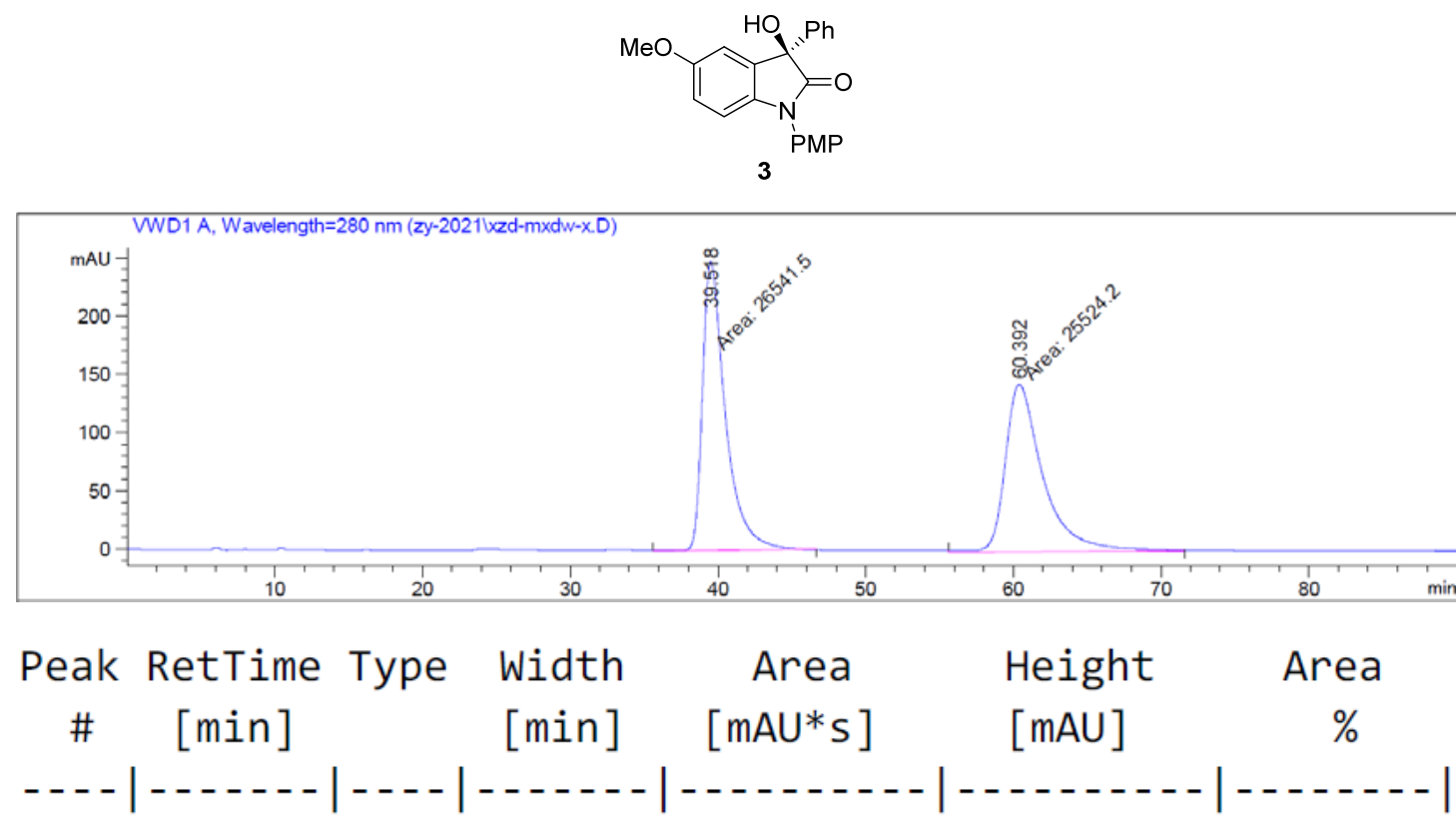

$\begin{array}{llllll}1 & 39.518 \text { MM } & 1.7920 & 2.65415 \mathrm{e} 4 & 246.85039 & 50.9769\end{array}$

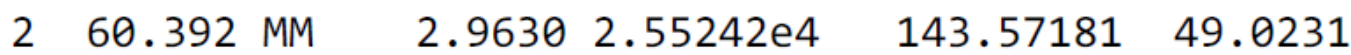



Peak RetTime Type width Area Height Area

\# $[\mathrm{min}] \quad[\mathrm{min}] \quad[\mathrm{mAU} * \mathrm{~s}] \quad[\mathrm{mAU}] \quad \%$

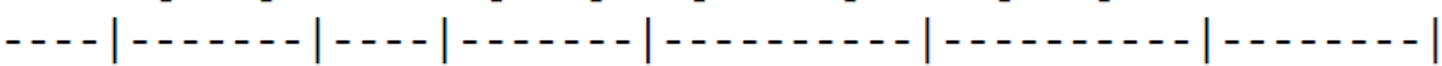

$1 \quad 39.515$ MM $\quad 1.8346 \quad 4.60044 \mathrm{e} 4 \quad 417.92371 \quad 97.7500$

$\begin{array}{lllllll}2 & 60.705 & \text { MM } & 2.3234 & 1058.93933 & 7.59620 & 2.2500\end{array}$ 
HPLC Spectra of 4.
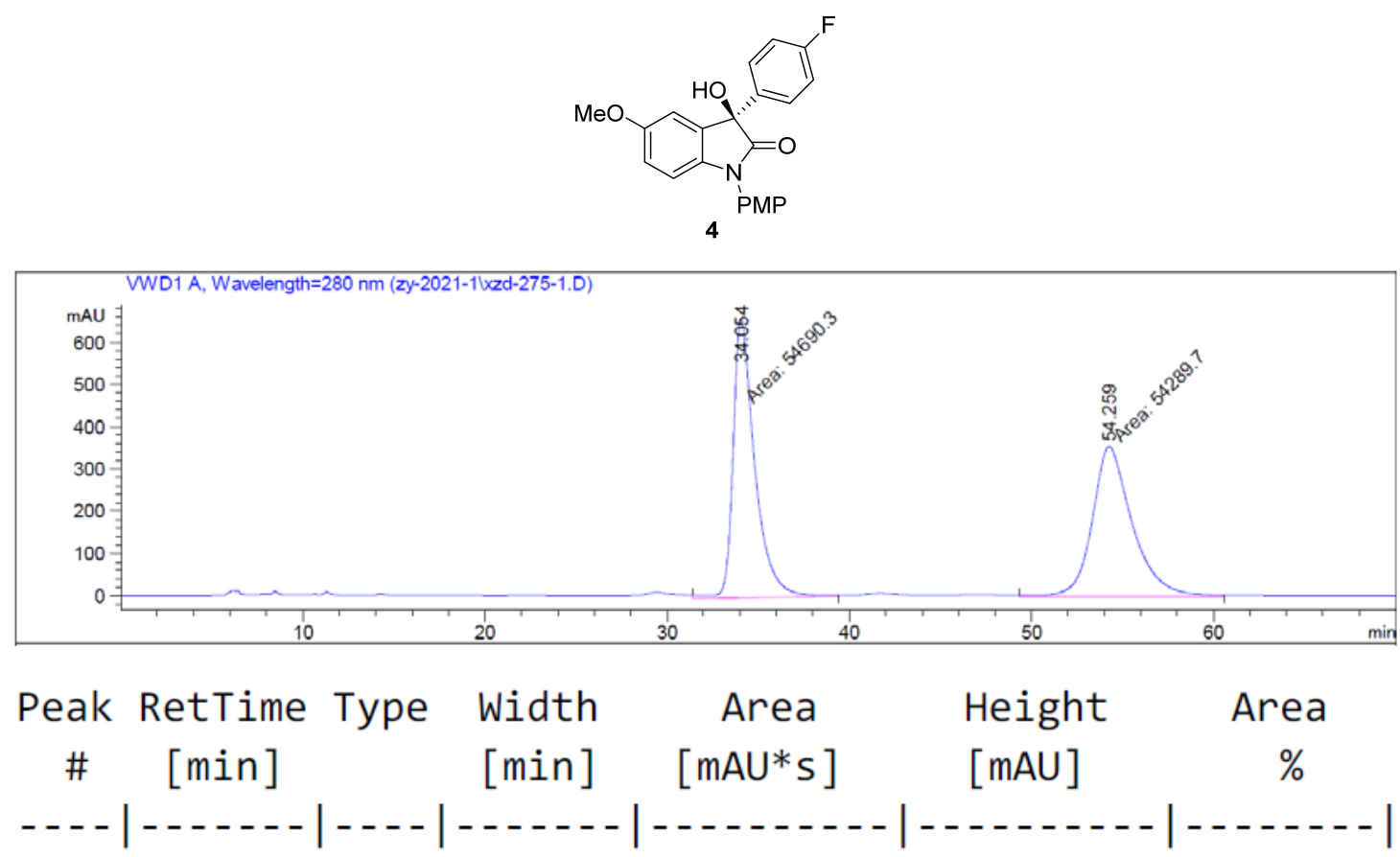
$134.054 \mathrm{MM}$
1.38175 .46903 e4 $\quad 659.69672 \quad 50.1838$

$254.259 \mathrm{MM}$

$2.5515 \quad 5.42897 \mathrm{e} 4$

$354.63116 \quad 49.8162$

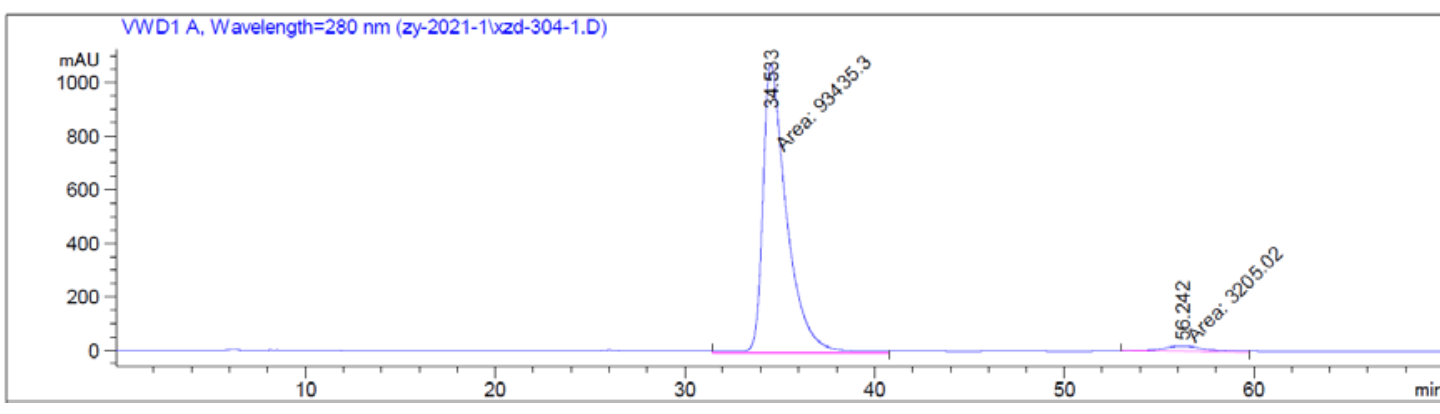

Peak RetTime Type Width Area Height Area

\# [min] [min $][\mathrm{mAU} * \mathrm{~s}] \quad[\mathrm{mAU}] \quad \%$

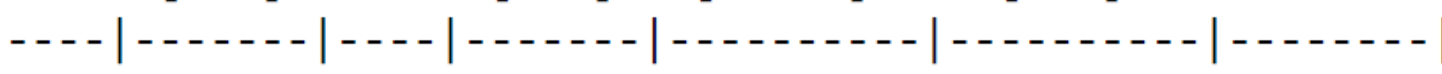

134.533 MM $\quad 1.4438 \quad 9.34353 \mathrm{e} 4 \quad 1078.60315 \quad 96.6836$

$2 \quad 56.242$ MM $2.6394 \quad 3205.02368 \quad 20.23813 \quad 3.3164$ 
HPLC Spectra of 5.
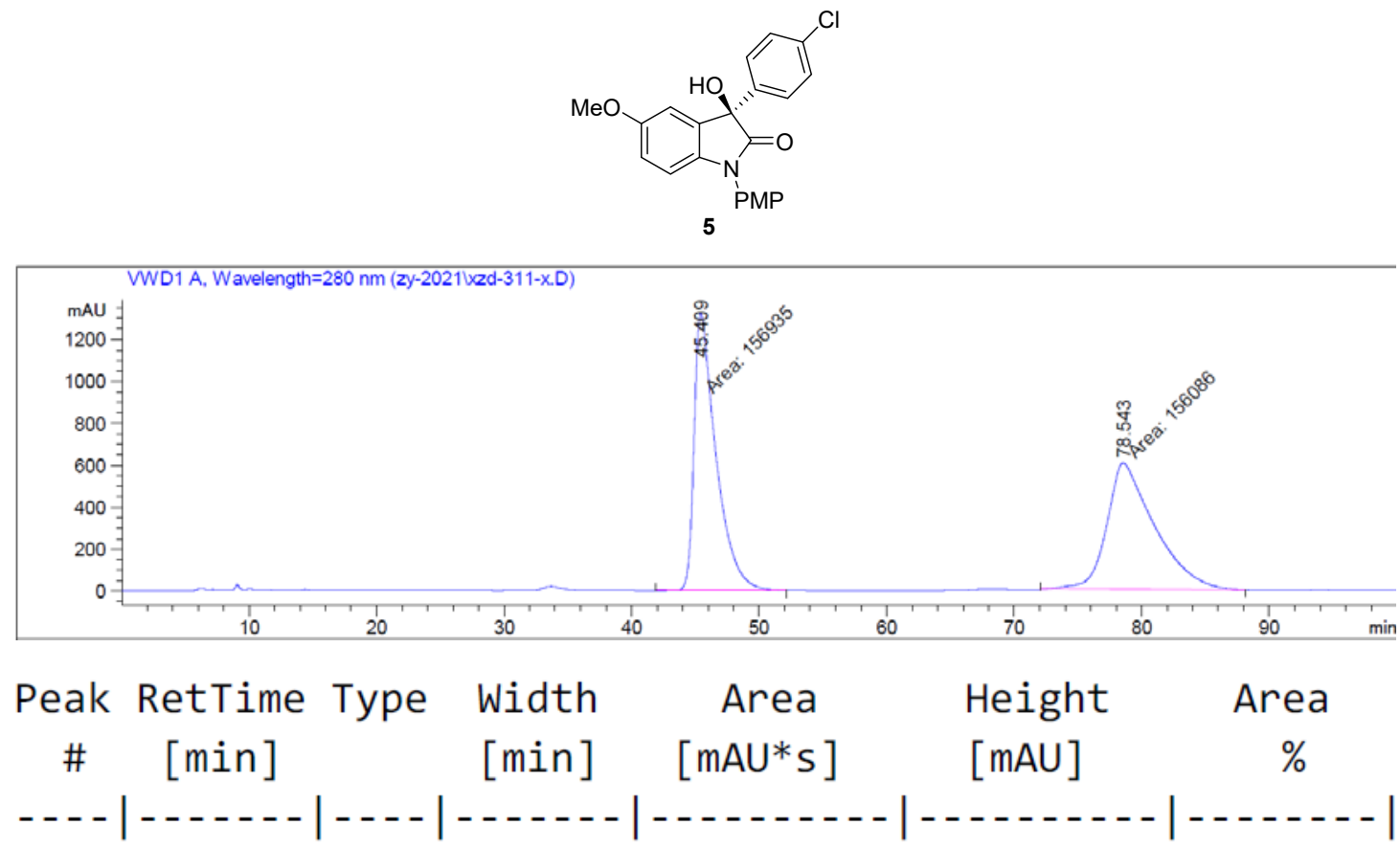

$\begin{array}{llllll}1 & 45.409 \text { MM } & 1.9823 & 1.56935 \mathrm{e} 5 & 1319.49097 & 50.1357\end{array}$

$2 \quad 78.543$ MM $4.32261 .56086 \mathrm{e} 5 \quad 601.82544 \quad 49.8643$

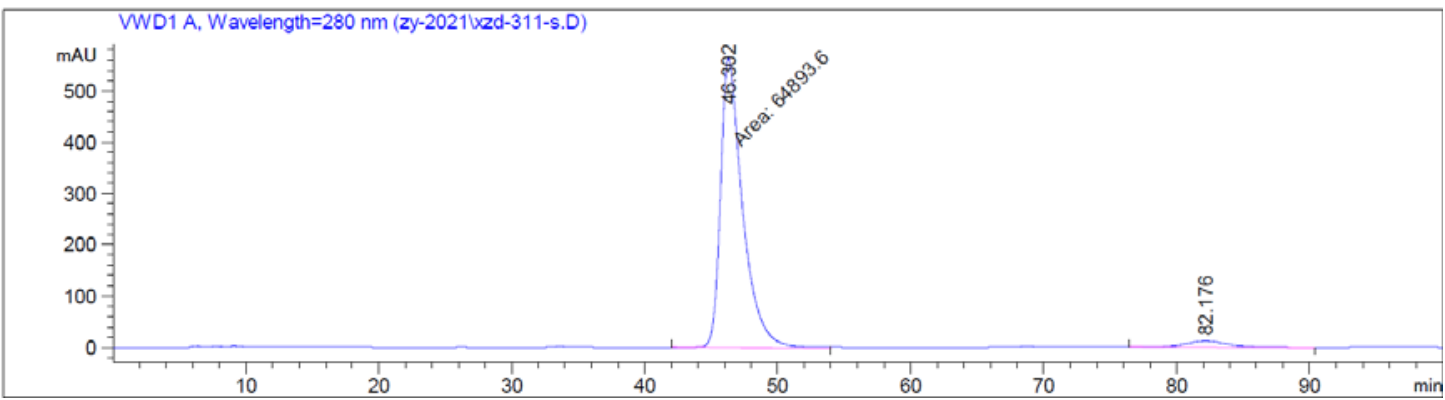

Peak RetTime Type Width Area Height Area

$\left.\begin{array}{llll}\# & {[\mathrm{~min}]} & {[\mathrm{min}]} & {[\mathrm{mAU} * \mathrm{~s}]}\end{array}\right]$

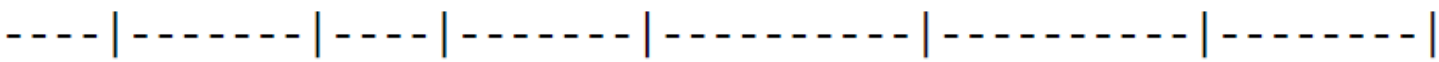

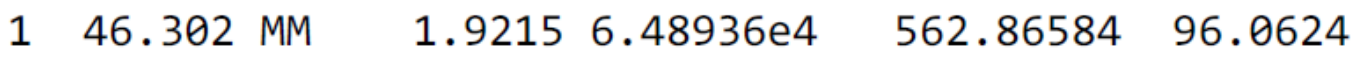

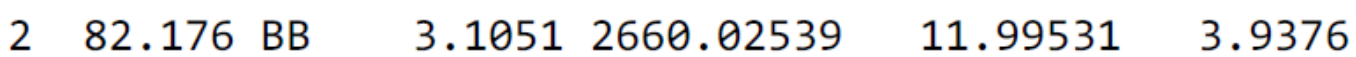


HPLC Spectra of 6.
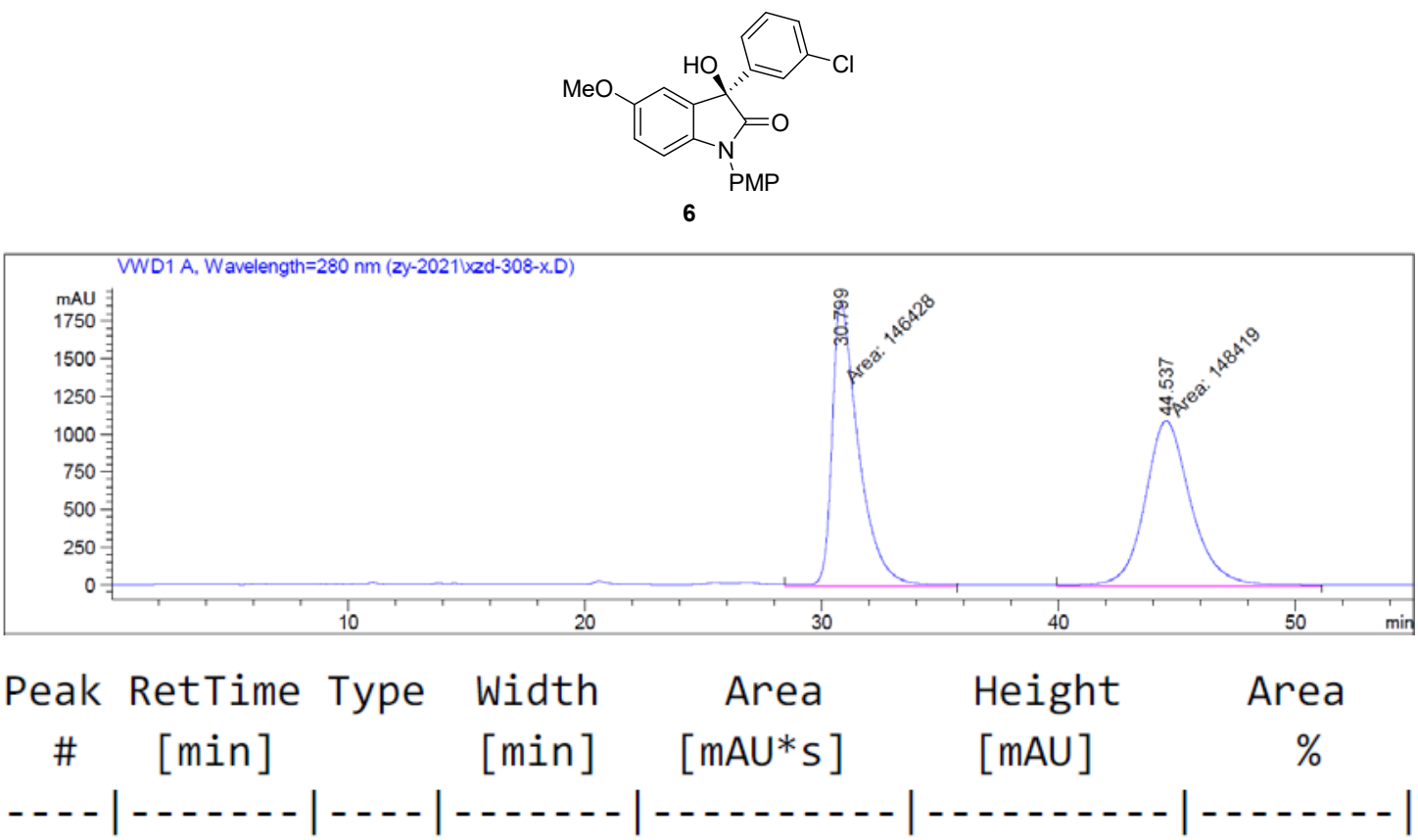

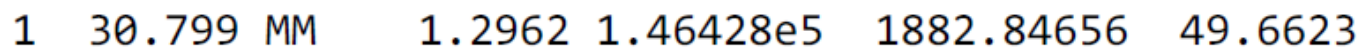

$\begin{array}{lllllll}2 & 44.537 \text { MM } & 2.2617 & 1.48419 \mathrm{e} 5 & 1093.69189 & 50.3377\end{array}$

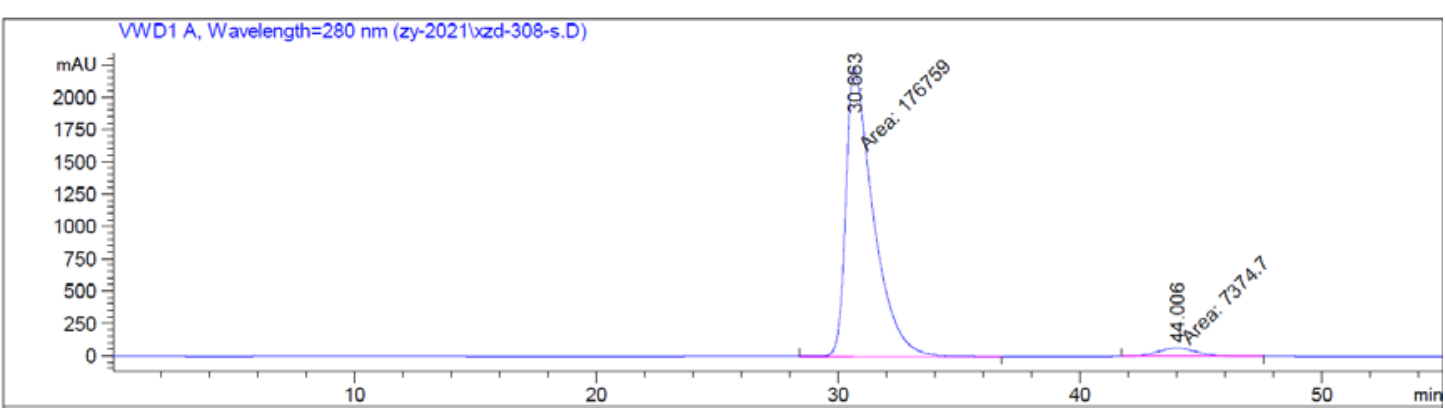

Peak RetTime Type Width Area Height Area

$\begin{array}{llll}\# & {[\mathrm{~min}]} & {[\mathrm{min}]} & {[\mathrm{mAU} * \mathrm{~s}]}\end{array}$

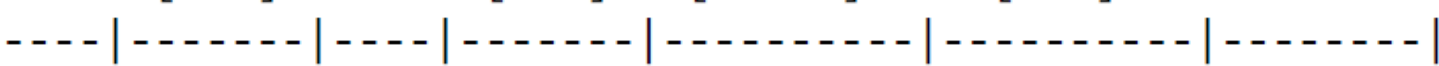

$\begin{array}{lllllll}1 & 30.663 \mathrm{MM} & 1.3187 & 1.76759 \mathrm{e} 5 & 2234.02515 & 95.9949\end{array}$

$\begin{array}{llllll}2 & 44.006 \text { MM } & 1.9314 & 7374.70361 & 63.63721 & 4.0051\end{array}$ 
HPLC Spectra of 7.
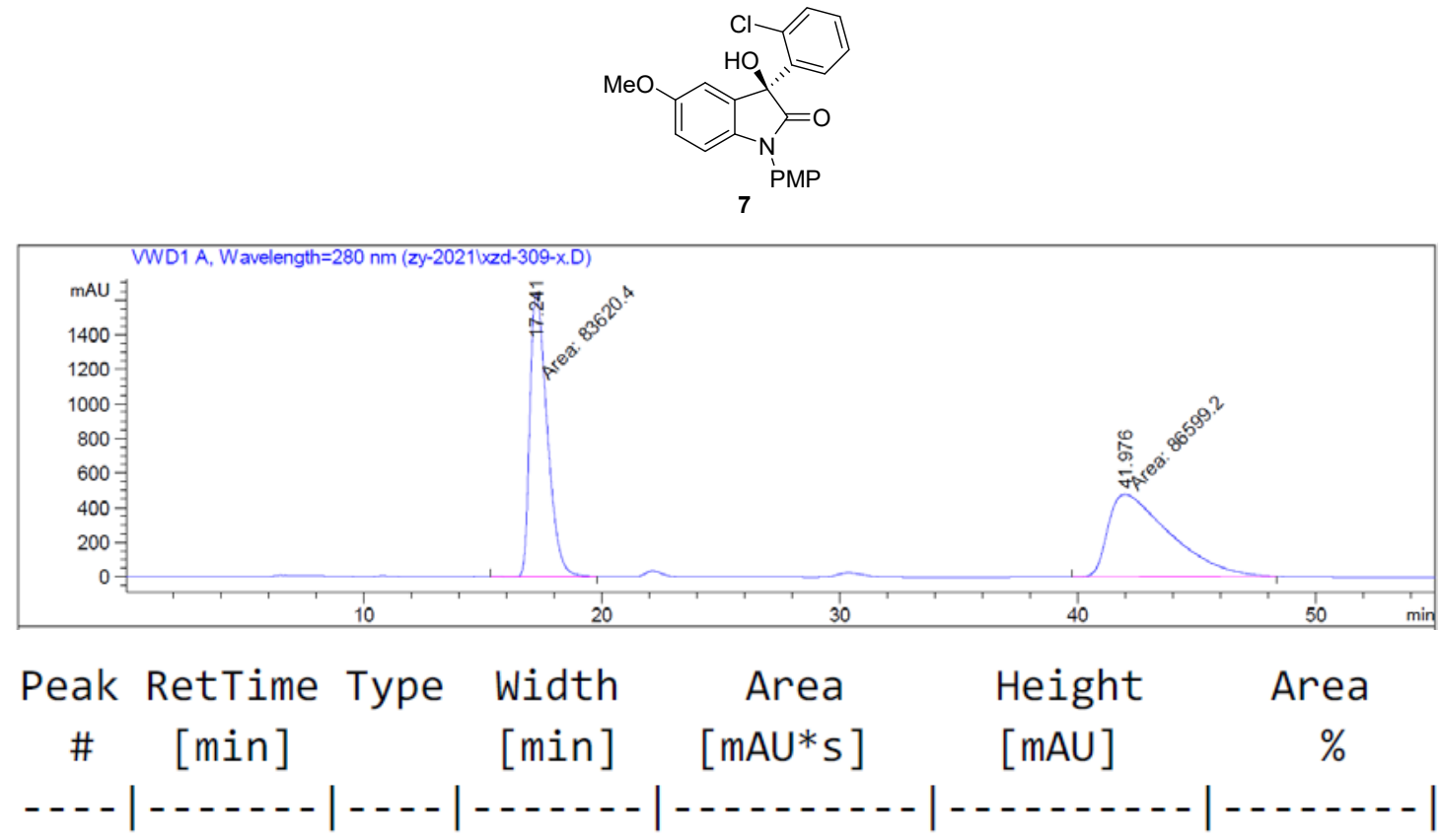
$117.241 \mathrm{MM}$
$0.8483 \quad 8.36204 \mathrm{e} 4 \quad 1642.94531 \quad 49.1250$
$241.976 \mathrm{MM}$
$3.01858 .65992 \mathrm{e} 4$
$478.15610 \quad 50.8750$

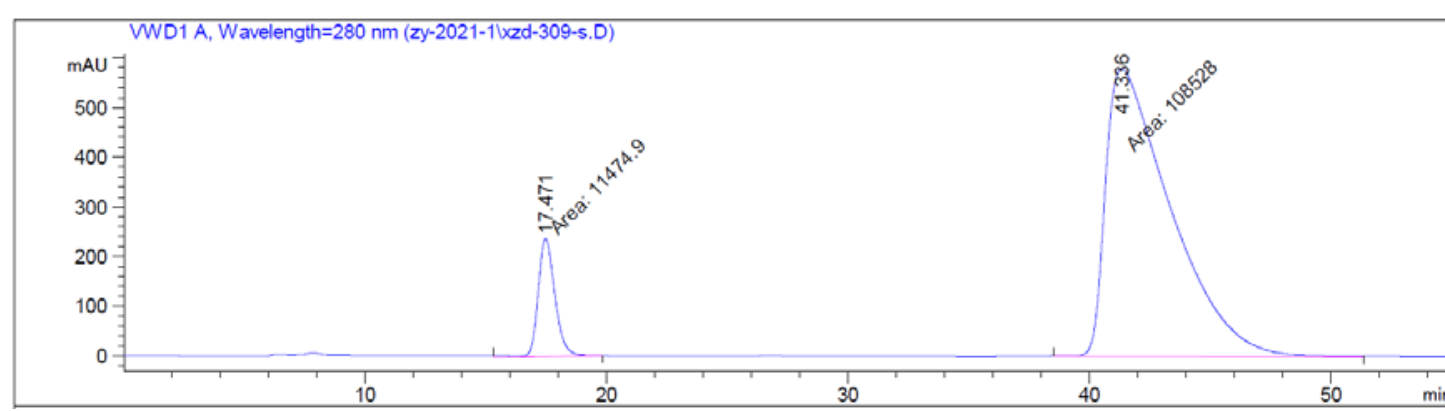

Peak RetTime Type Width Area Height Area

\# $[\mathrm{min}] \quad[\mathrm{min}] \quad[\mathrm{mAU} * \mathrm{~s}] \quad[\mathrm{mAU}] \quad \%$

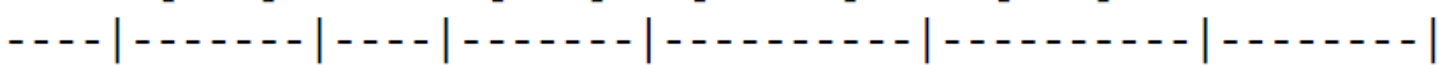
$117.471 \mathrm{MM}$
$0.80631 .14749 \mathrm{e} 4$
237.19571
9.5622
$241.336 \mathrm{MM}$
$3.11911 .08528 \mathrm{e} 5$
579.91498
90.4378 
HPLC Spectra of 8 .
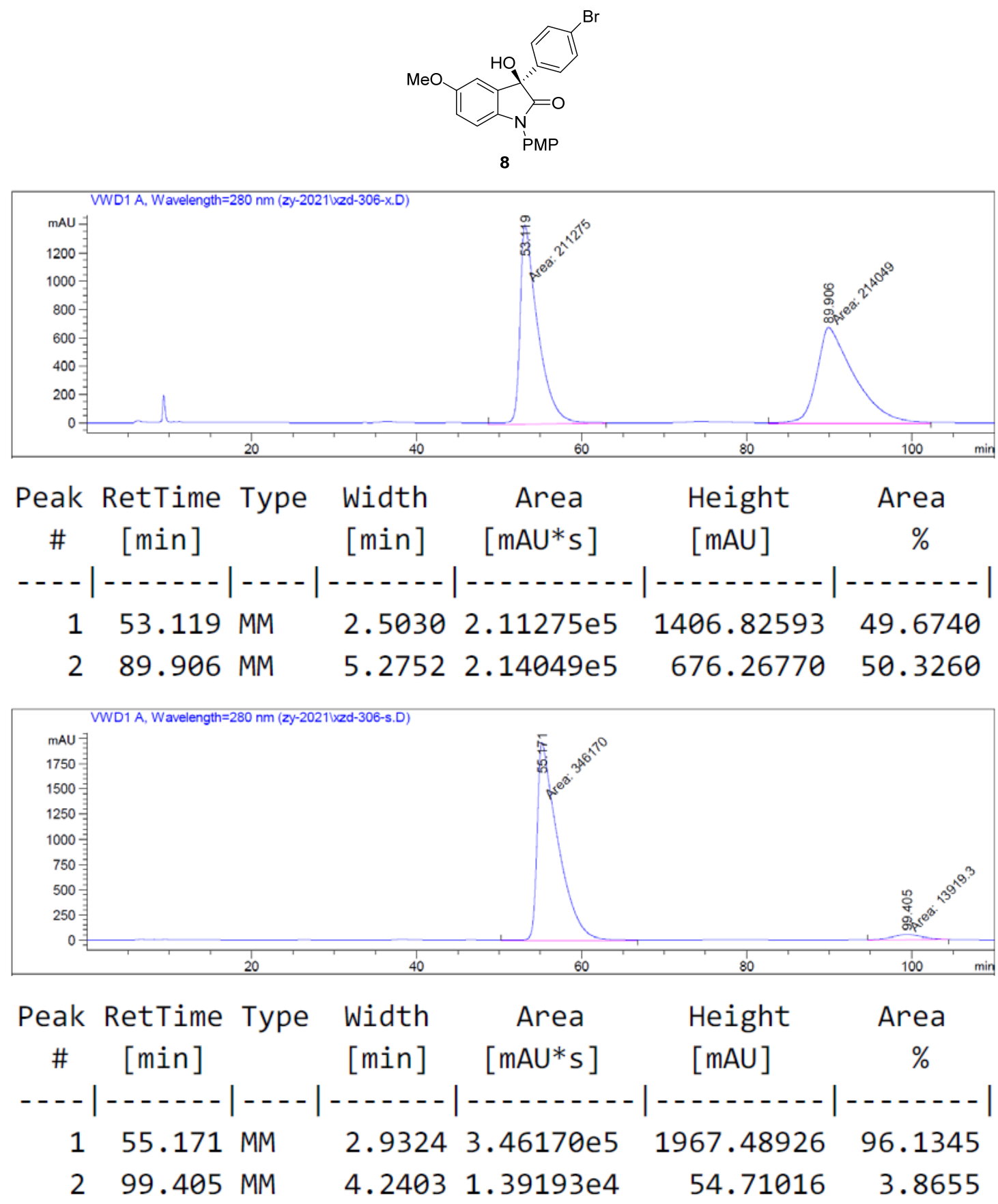
HPLC Spectra of 9.
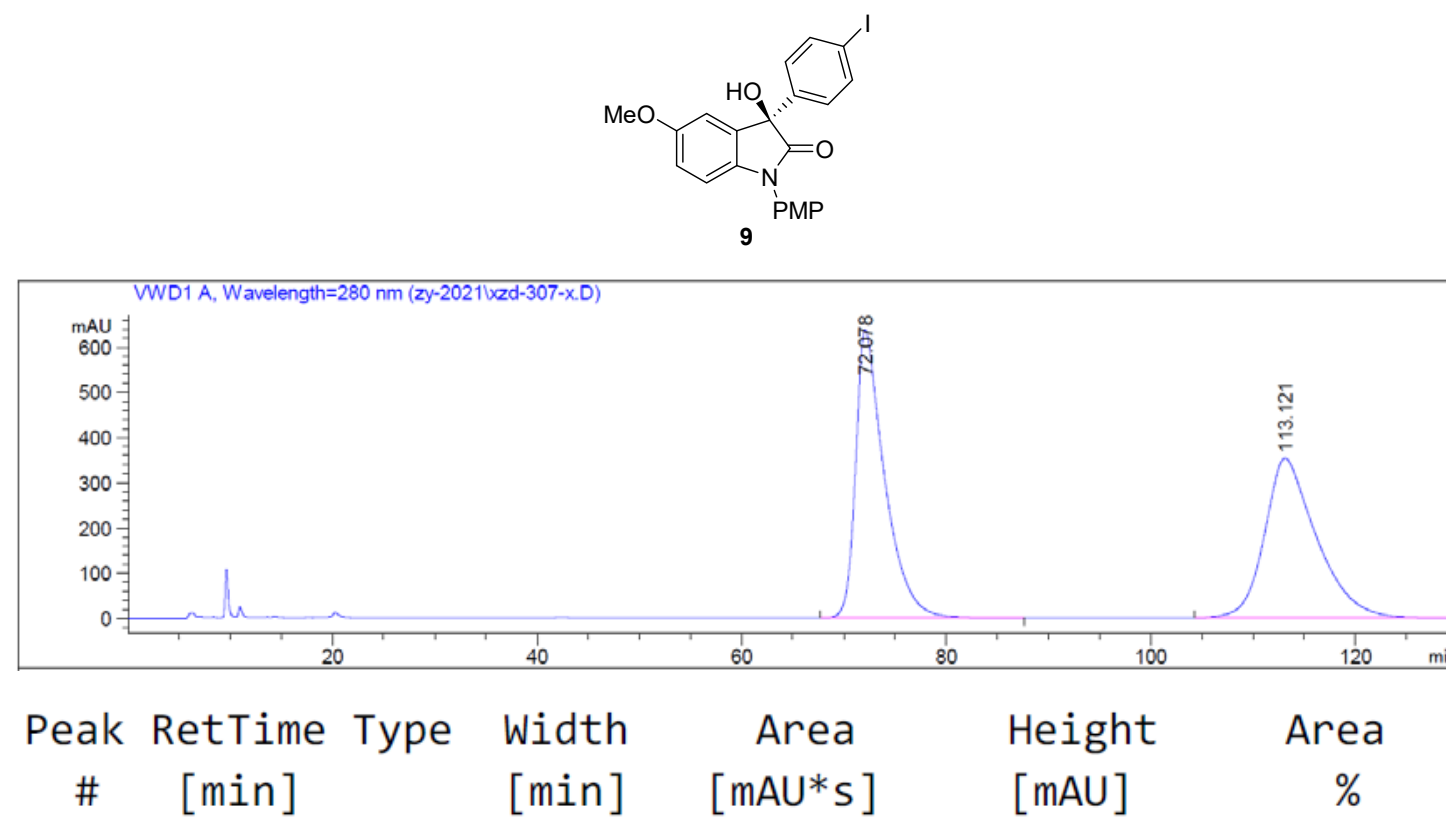

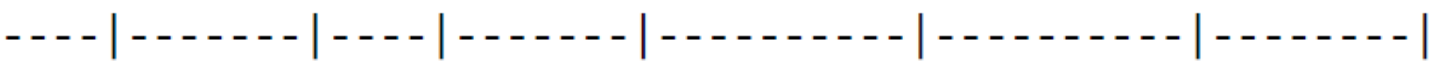

$$
\begin{aligned}
& \begin{array}{lllllll}
1 & 72.078 & \text { BB } & 2.8199 & 1.25374 \mathrm{e} 5 & 638.76294 & 49.9938
\end{array} \\
& \begin{array}{llllll}
2 & 113.121 \text { BB } \quad 5.0452 & 1.25405 \mathrm{e} 5 & 353.97415 & 50.0062
\end{array}
\end{aligned}
$$

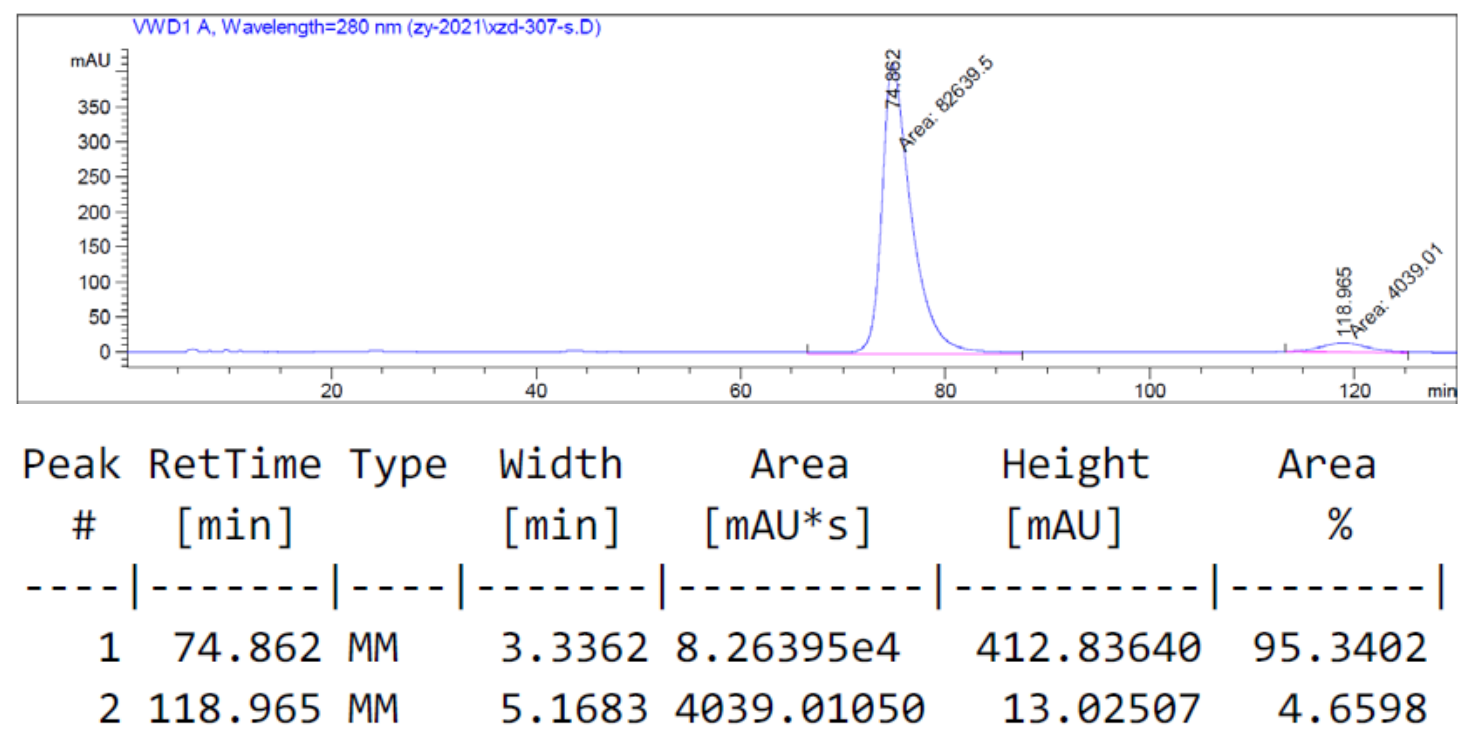


HPLC Spectra of $\mathbf{1 0 .}$
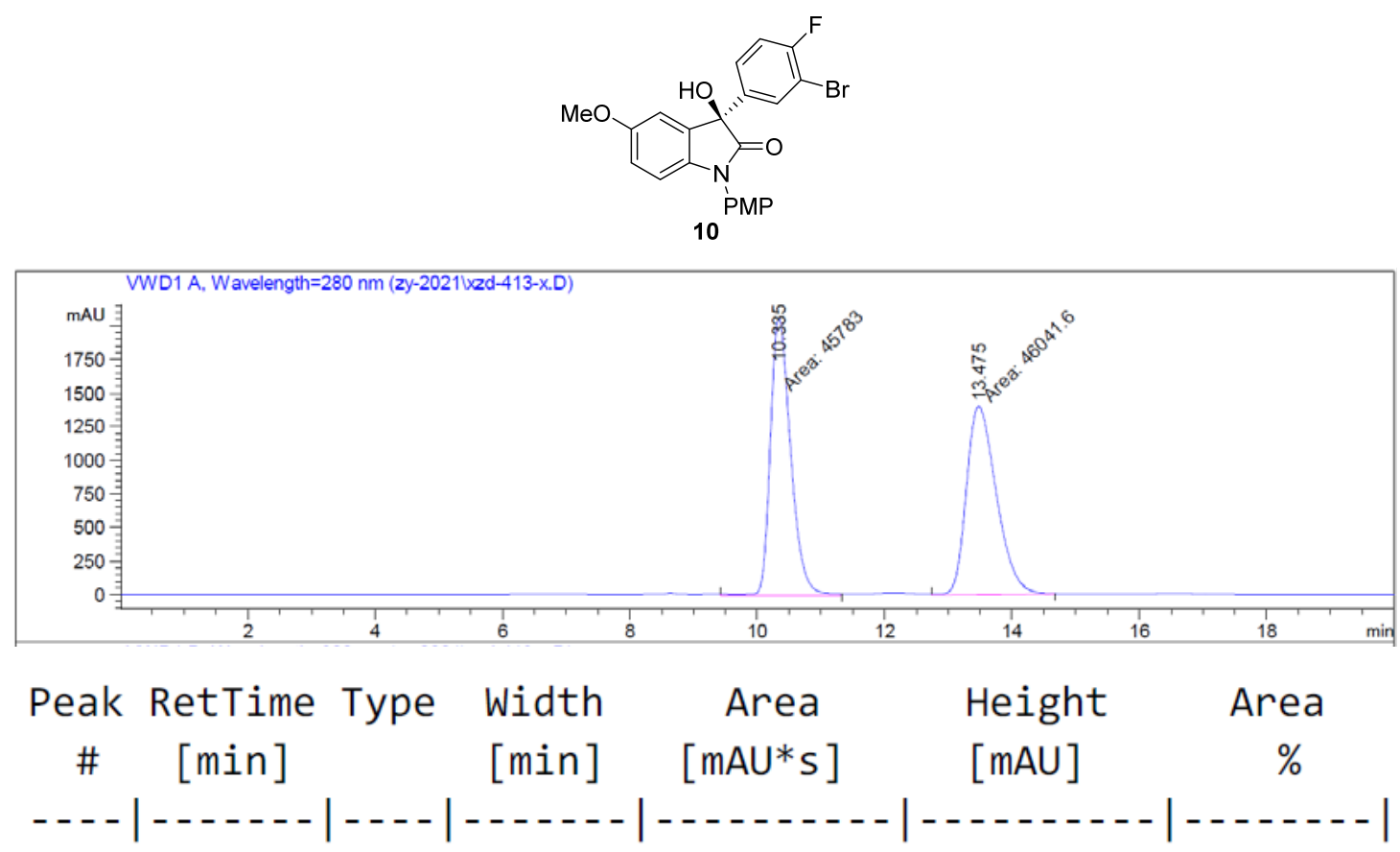

$1 \quad 10.335$ MM $\quad 0.3694 \quad 4.57830 \mathrm{e} 4 \quad 2065.81714 \quad 49.8592$

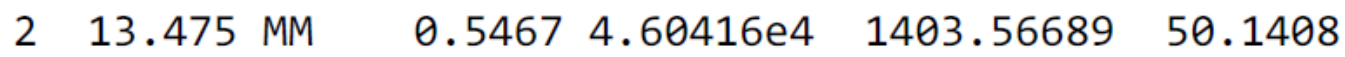

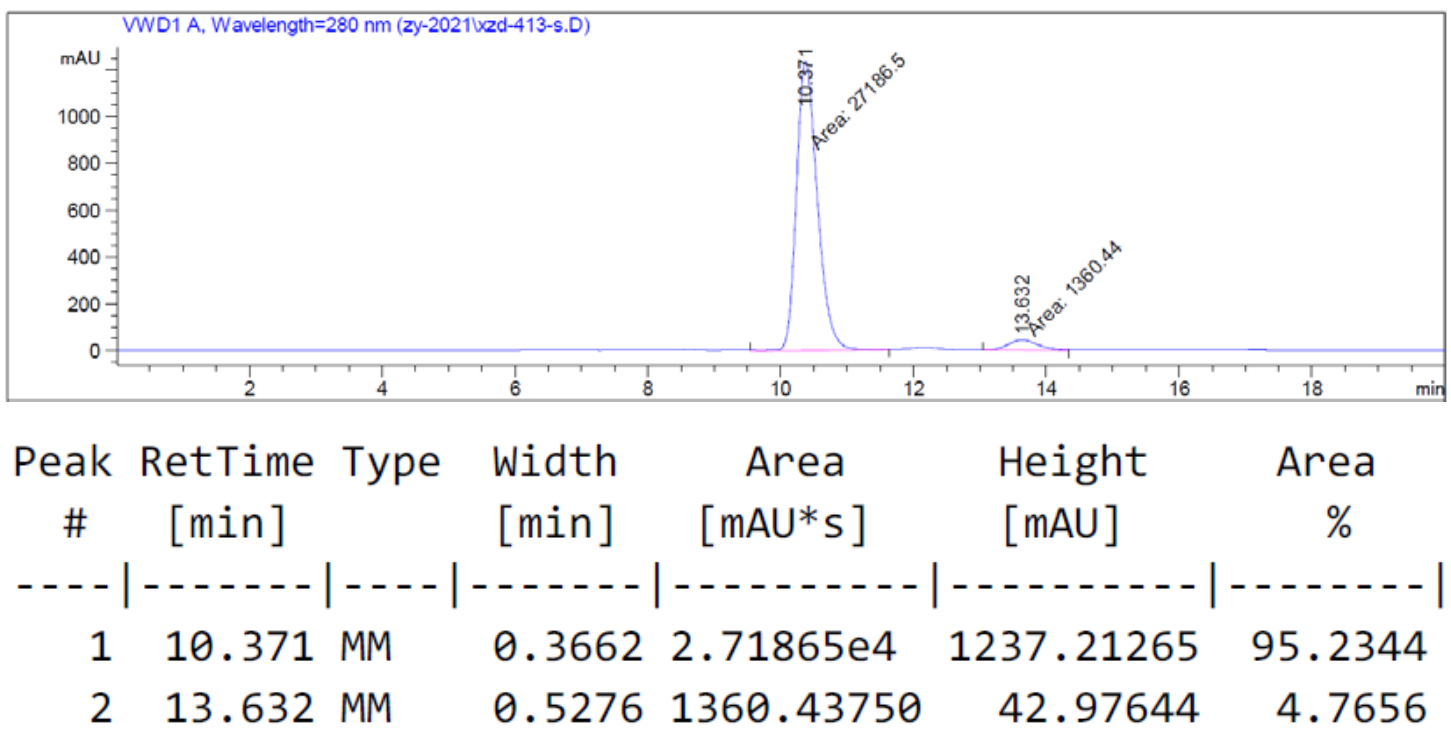


HPLC Spectra of 11.
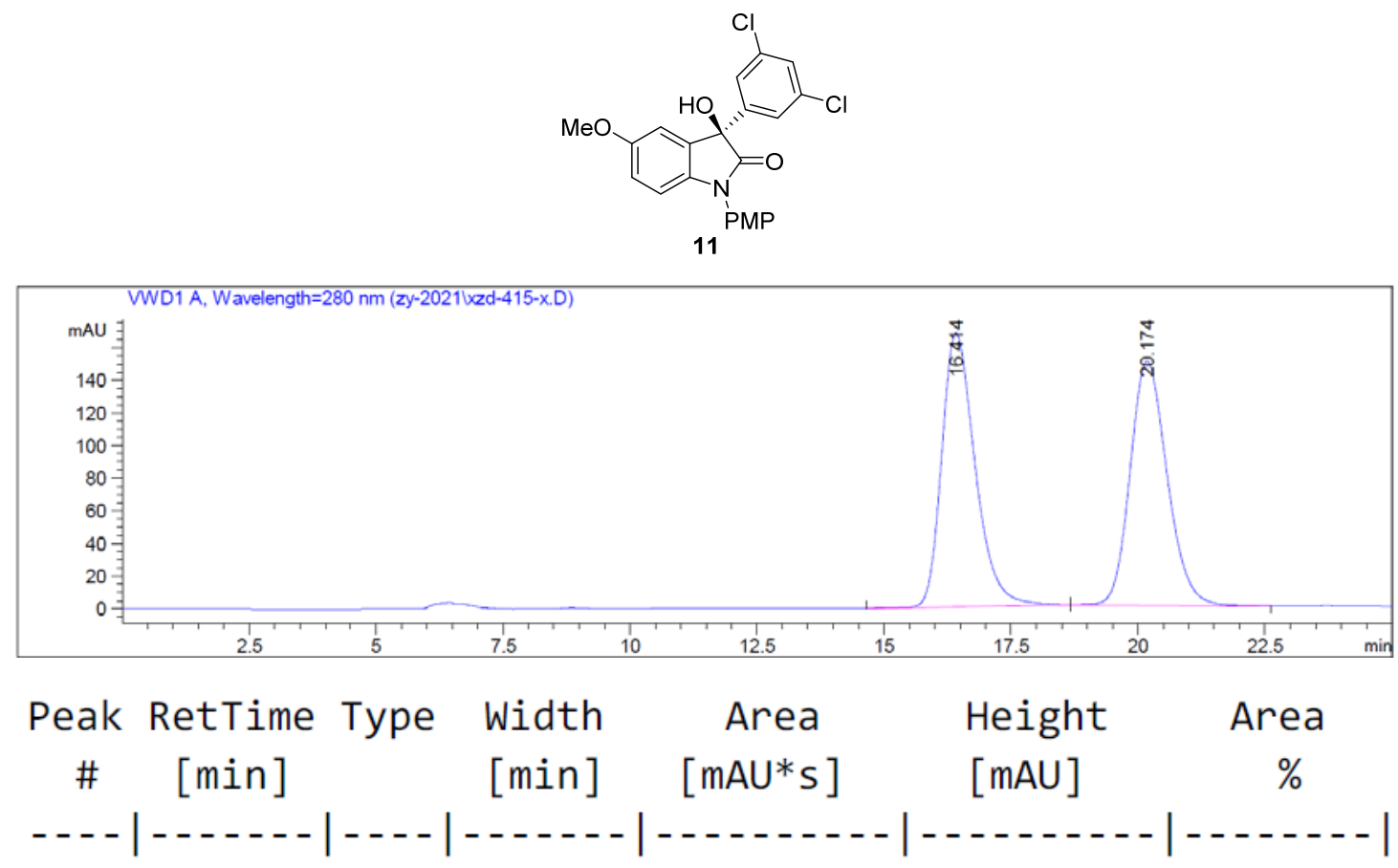

$\begin{array}{lllllll}1 & 16.414 & \text { BB } & 0.7012 & 7668.28809 & 167.78581 & 50.3282\end{array}$

$\begin{array}{lllllll}2 & 20.174 & \text { BB } & 0.7816 & 7568.26416 & 149.76472 & 49.6718\end{array}$

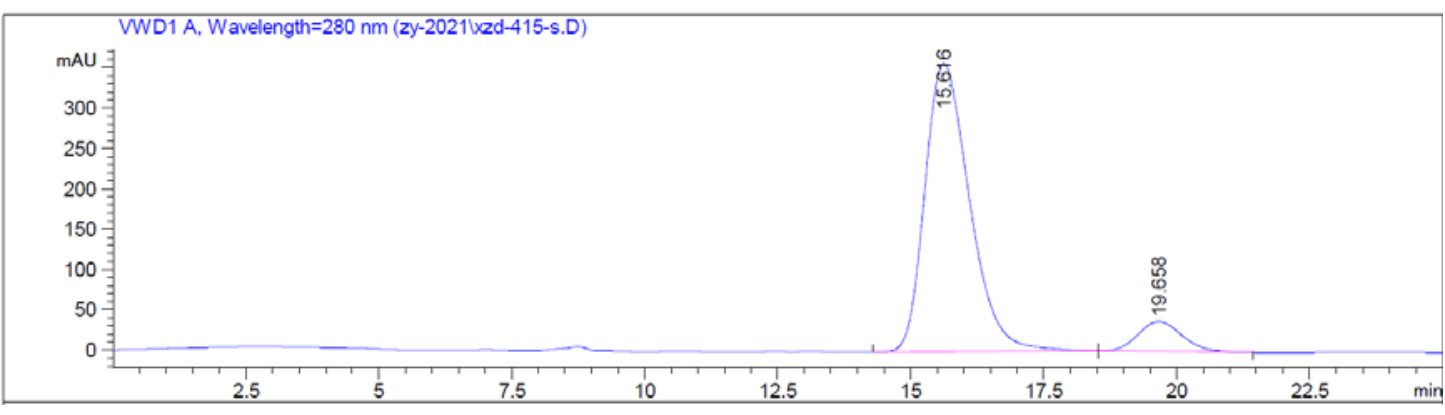

Peak RetTime Type Width Area Height Area

\# $[\mathrm{min}] \quad[\mathrm{min}] \quad[\mathrm{mAU} * \mathrm{~s}] \quad[\mathrm{mAU}] \quad \%$

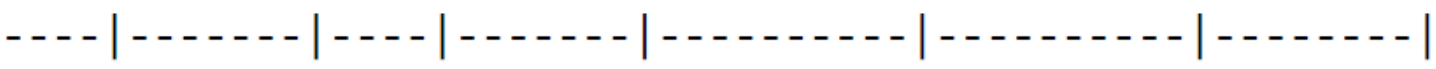

$\begin{array}{lllllll}1 & 15.616 & \text { BB } & 0.9064 & 2.08834 \mathrm{e} 4 & 357.01459 & 90.7497\end{array}$

$\begin{array}{lllllll}2 & 19.658 & \text { BВ } & 0.8959 & 2128.69678 & 37.07051 & 9.2503\end{array}$ 
HPLC Spectra of $\mathbf{1 2 .}$
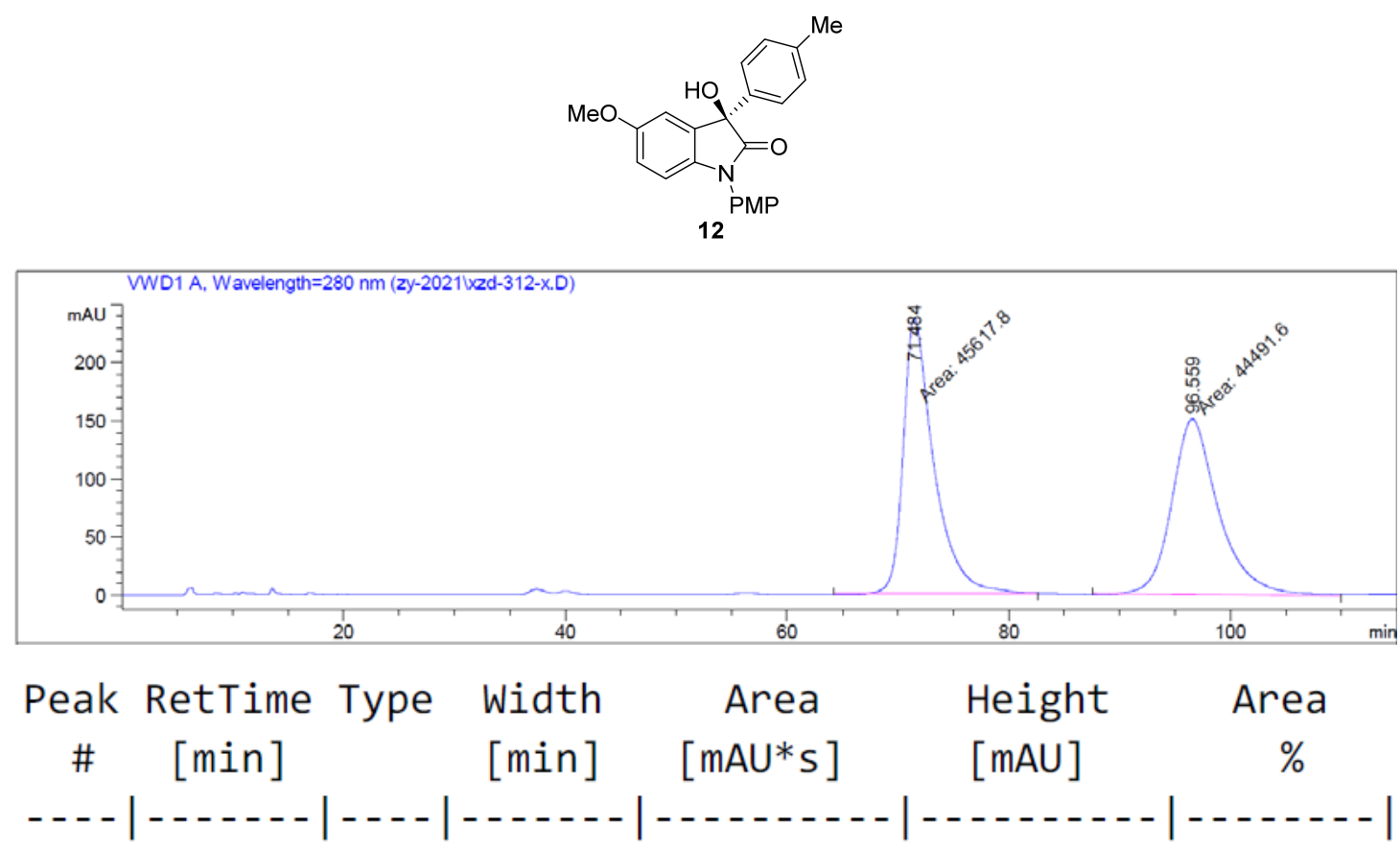

$\begin{array}{lllllll}1 & 71.484 \text { MM } & 3.2016 & 4.56178 \mathrm{e} 4 & 237.47266 & 50.6249\end{array}$

$\begin{array}{lllllll}2 & 96.559 \text { мM } & 4.9037 & 4.44916 \mathrm{e} 4 & 151.21700 & 49.3751\end{array}$

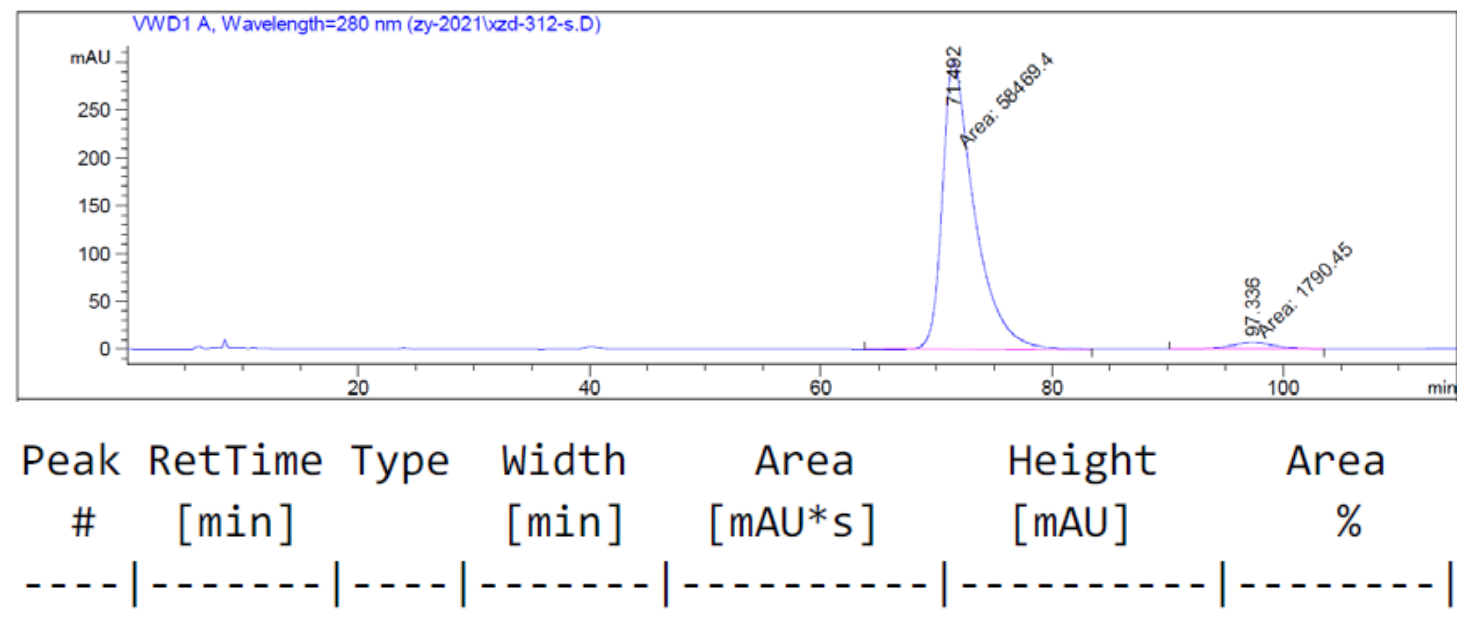

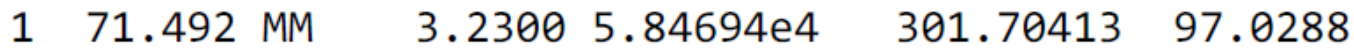

$\begin{array}{llllll}2 & 97.336 \text { MM } & 4.3343 & 1790.44543 & 6.88479 & 2.9712\end{array}$ 
HPLC Spectra of $\mathbf{1 3 .}$
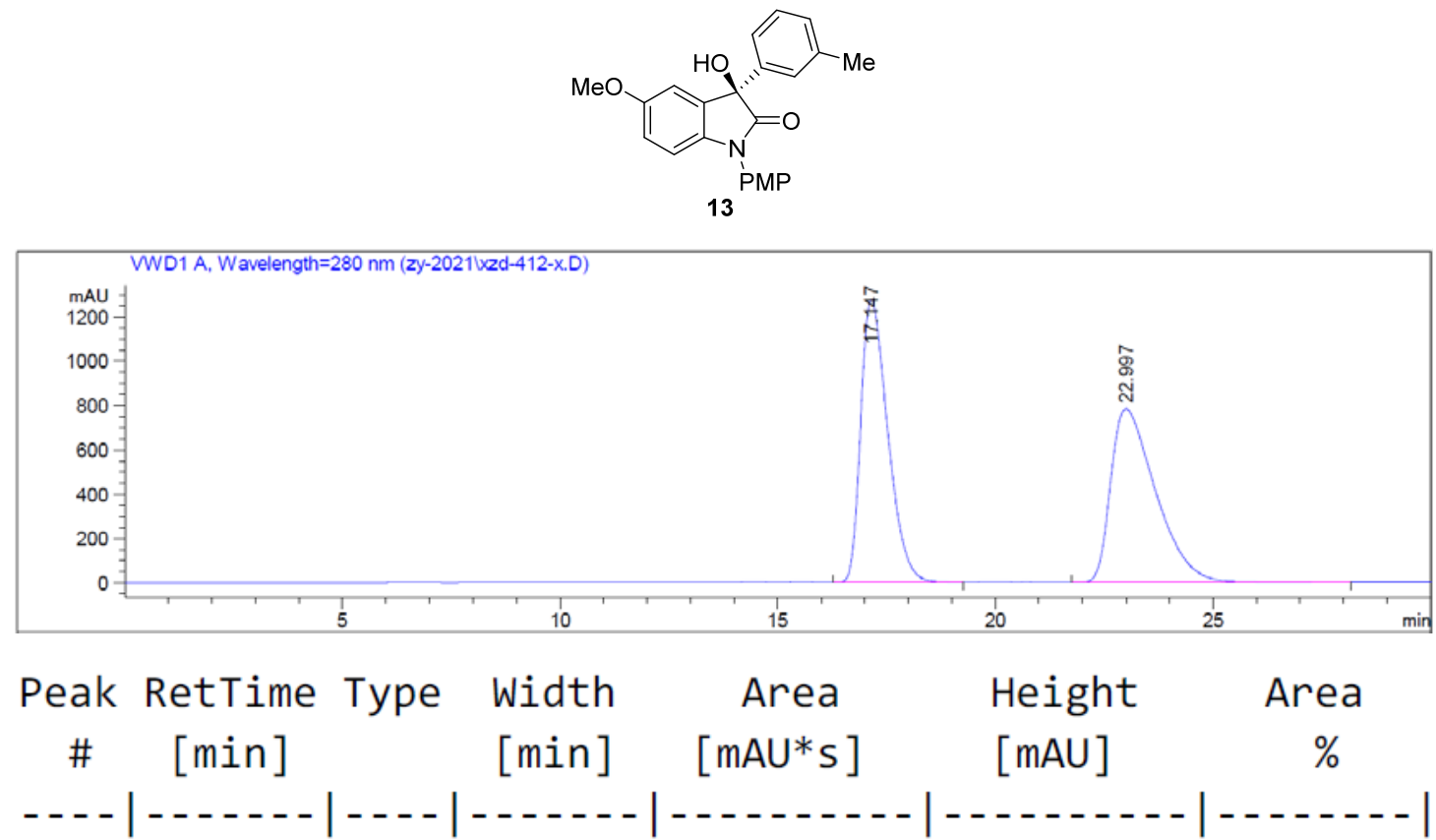
$117.147 \mathrm{BB}$
$\begin{array}{llll}0.6666 & 5.50517 \mathrm{e} 4 & 1277.86230 & 49.4417\end{array}$
$222.997 \mathrm{BB}$
$\begin{array}{llll}1.0956 & 5.62950 \mathrm{e} 4 & 784.08624 & 50.5583\end{array}$

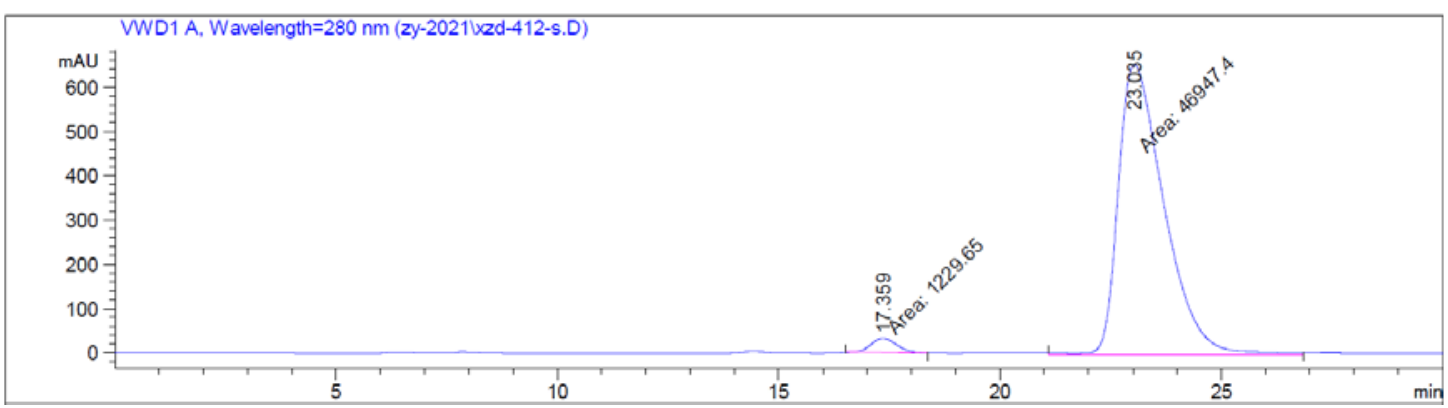

Peak RetTime Type Width Area Height Area \# $[\mathrm{min}] \quad[\mathrm{min}] \quad[\mathrm{mAU} * \mathrm{~s}] \quad[\mathrm{mAU}] \quad \%$

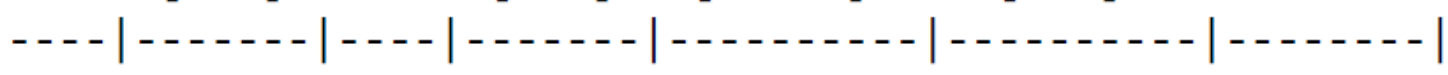

$\begin{array}{lllllll}1 & 17.359 & \text { MM } & 0.6503 & 1229.65137 & 31.51258 & 2.5524\end{array}$

$\begin{array}{llllll}2 & 23.035 \text { MM } & 1.1954 & 4.69474 \mathrm{e} 4 & 654.56140 & 97.4476\end{array}$ 
HPLC Spectra of 14.
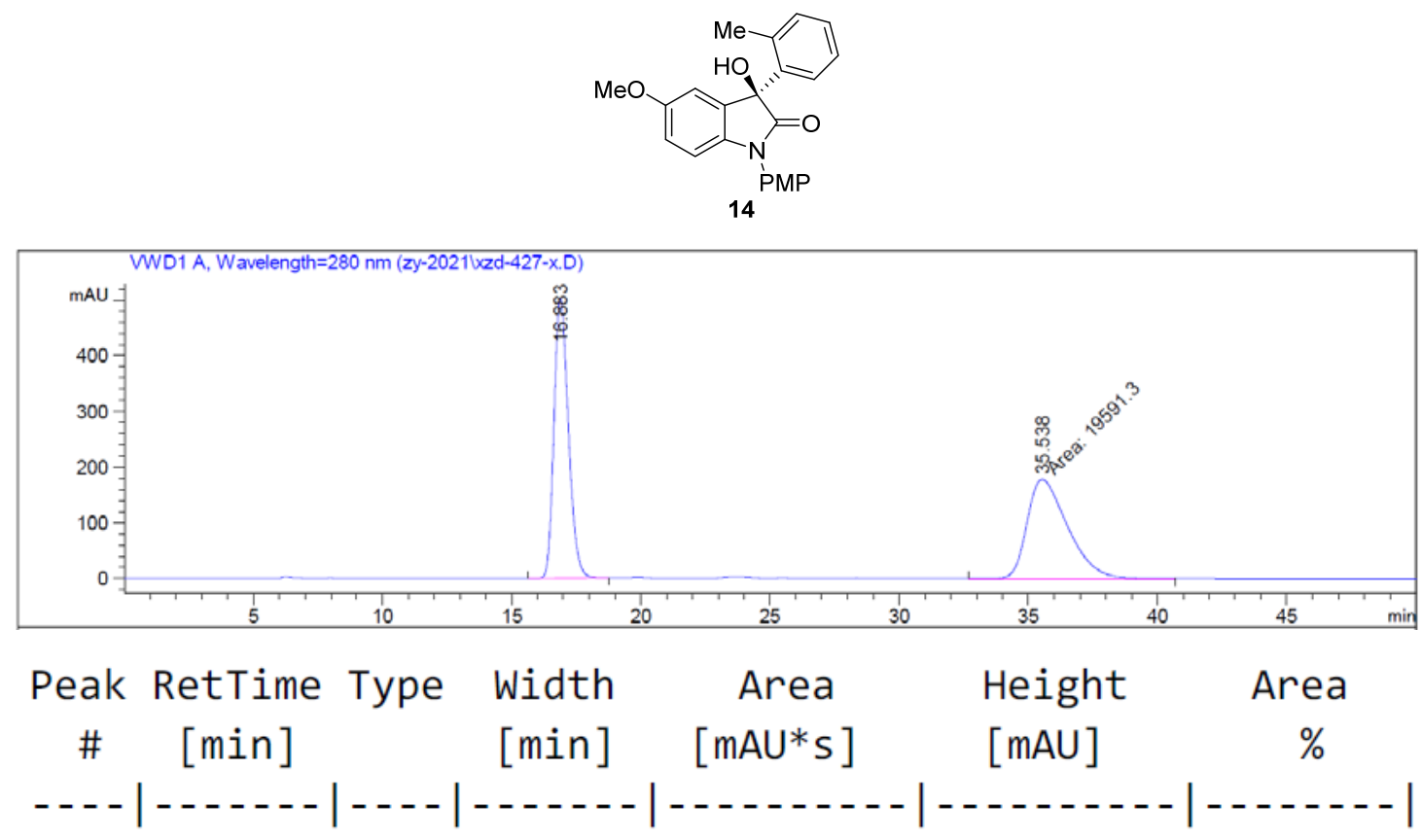

$\begin{array}{lllllll}1 & 16.883 & \text { BB } & 0.5981 & 1.94132 \mathrm{e} 4 & 503.15912 & 49.7717\end{array}$

$\begin{array}{lllllll}2 & 35.538 \text { MM } & 1.8290 & 1.95913 \mathrm{e} 4 & 178.52211 & 50.2283\end{array}$

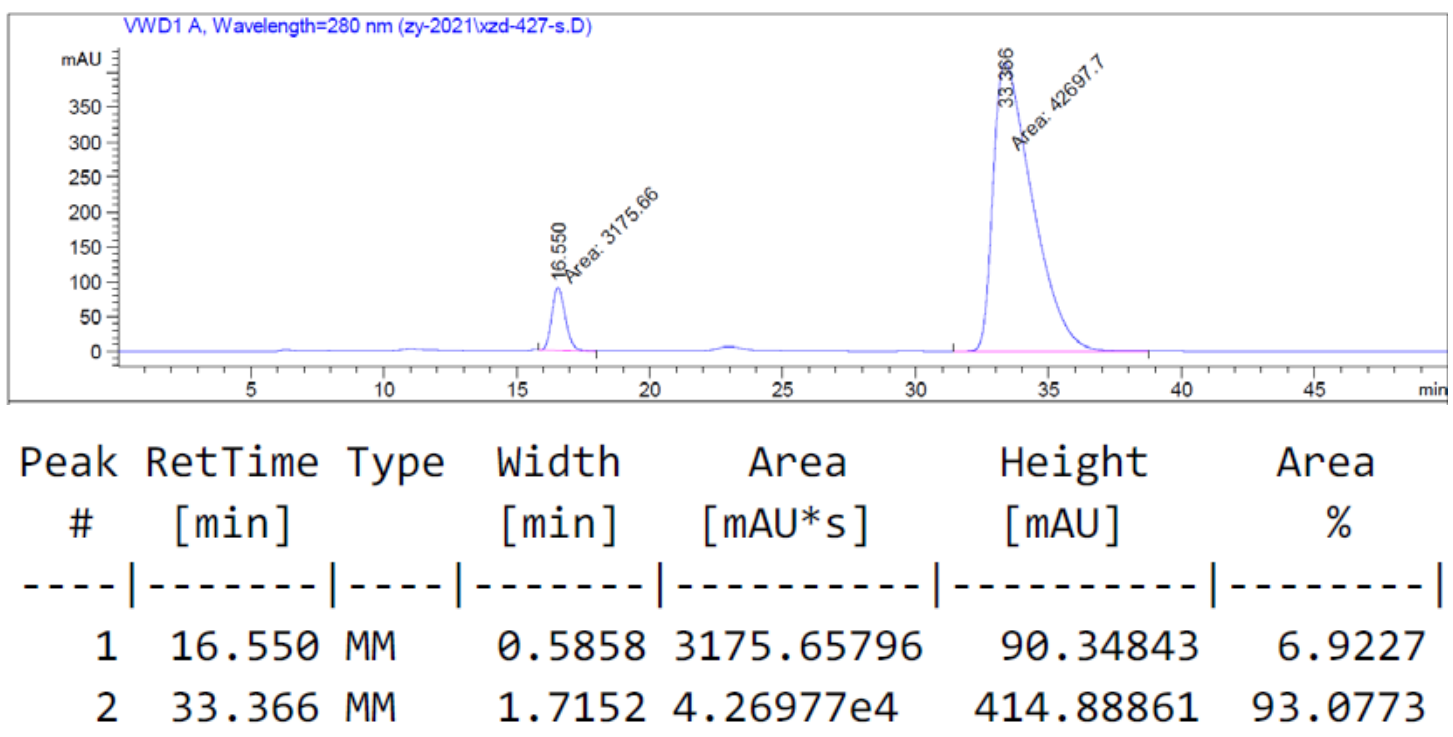


HPLC Spectra of $\mathbf{1 5 .}$
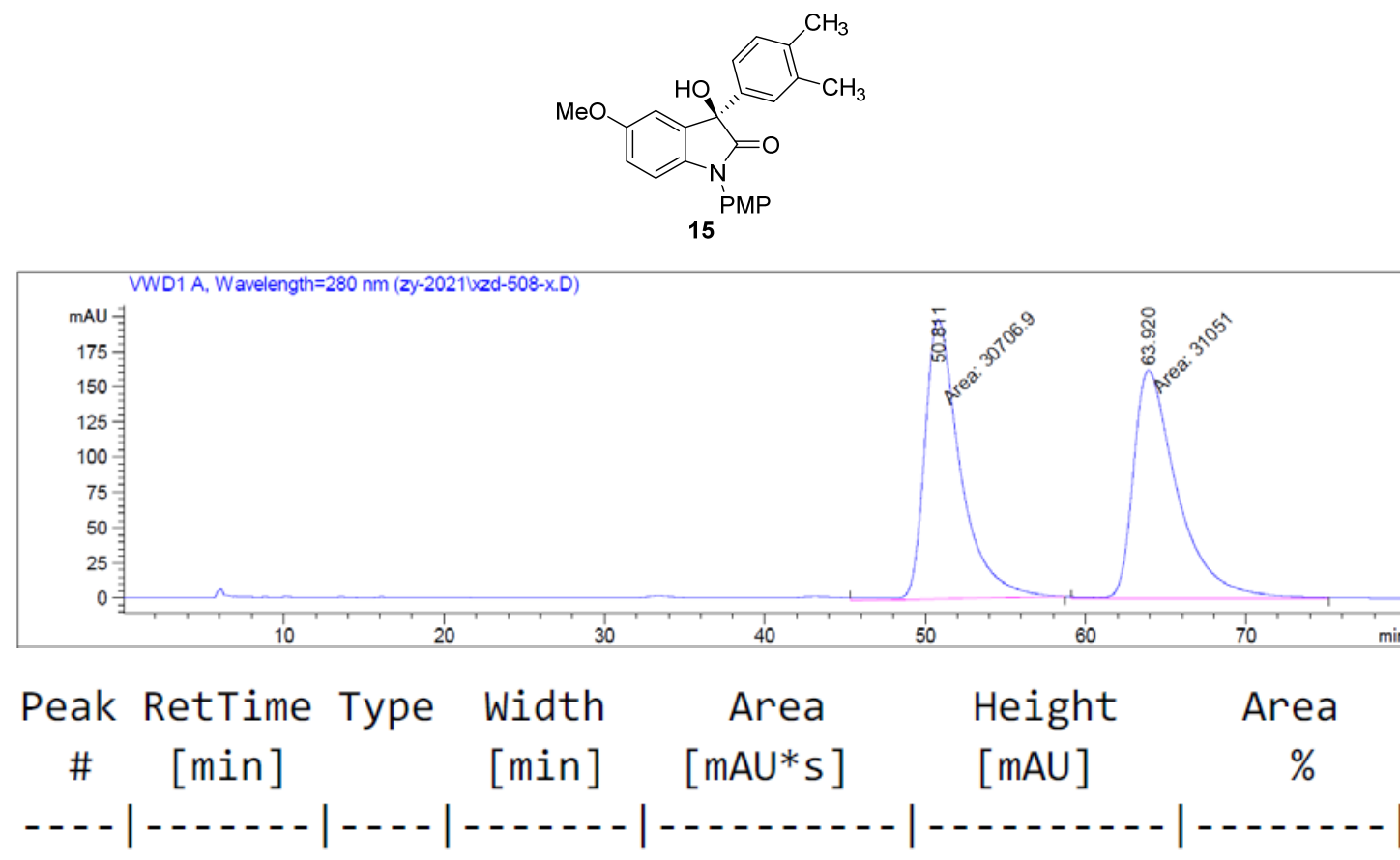

$\begin{array}{lllllll}1 & 50.811 & \text { MM } & 2.5811 & 3.07069 \mathrm{e} 4 & 198.27734 & 49.7214\end{array}$

$\begin{array}{lllllll}2 & 63.920 \text { MM } & 3.1984 & 3.10510 \mathrm{e} 4 & 161.80267 & 50.2786\end{array}$

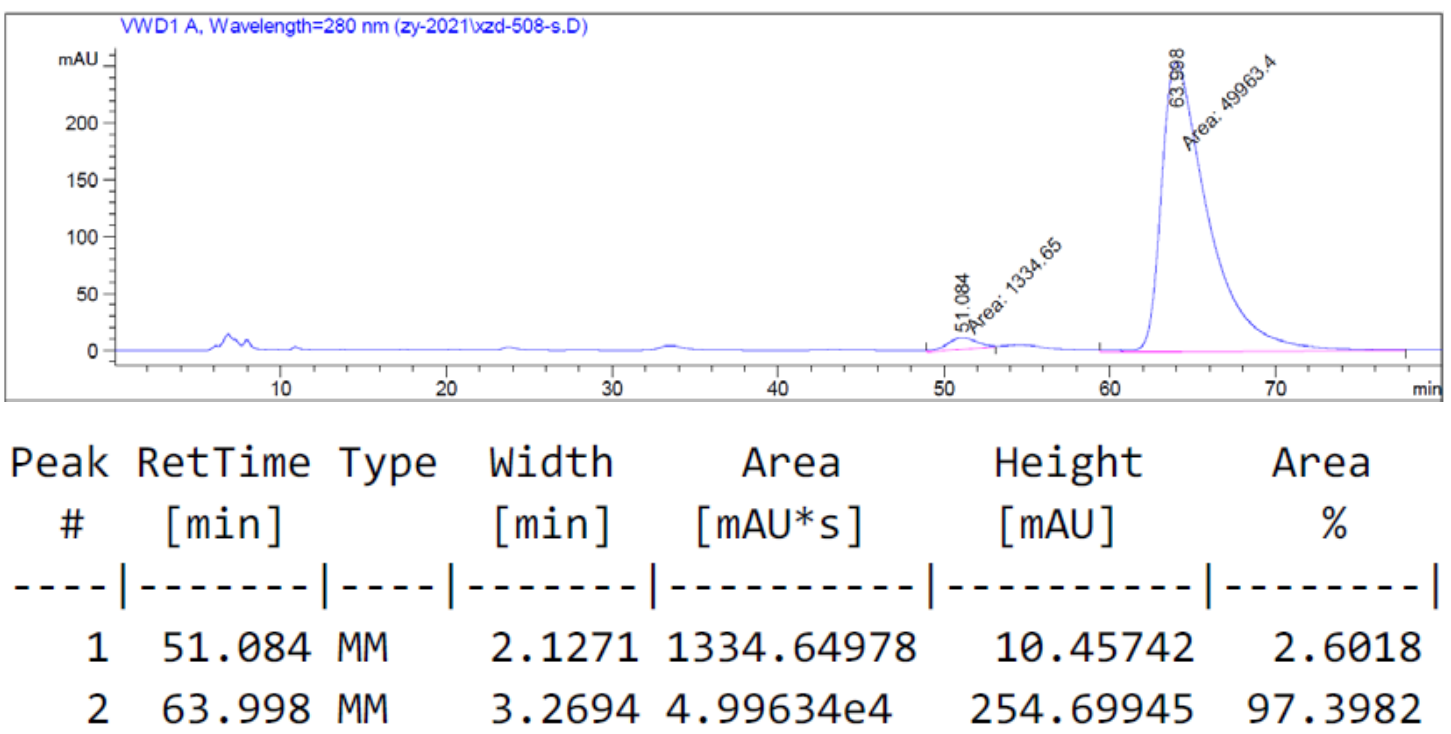


HPLC Spectra of $\mathbf{1 6 .}$
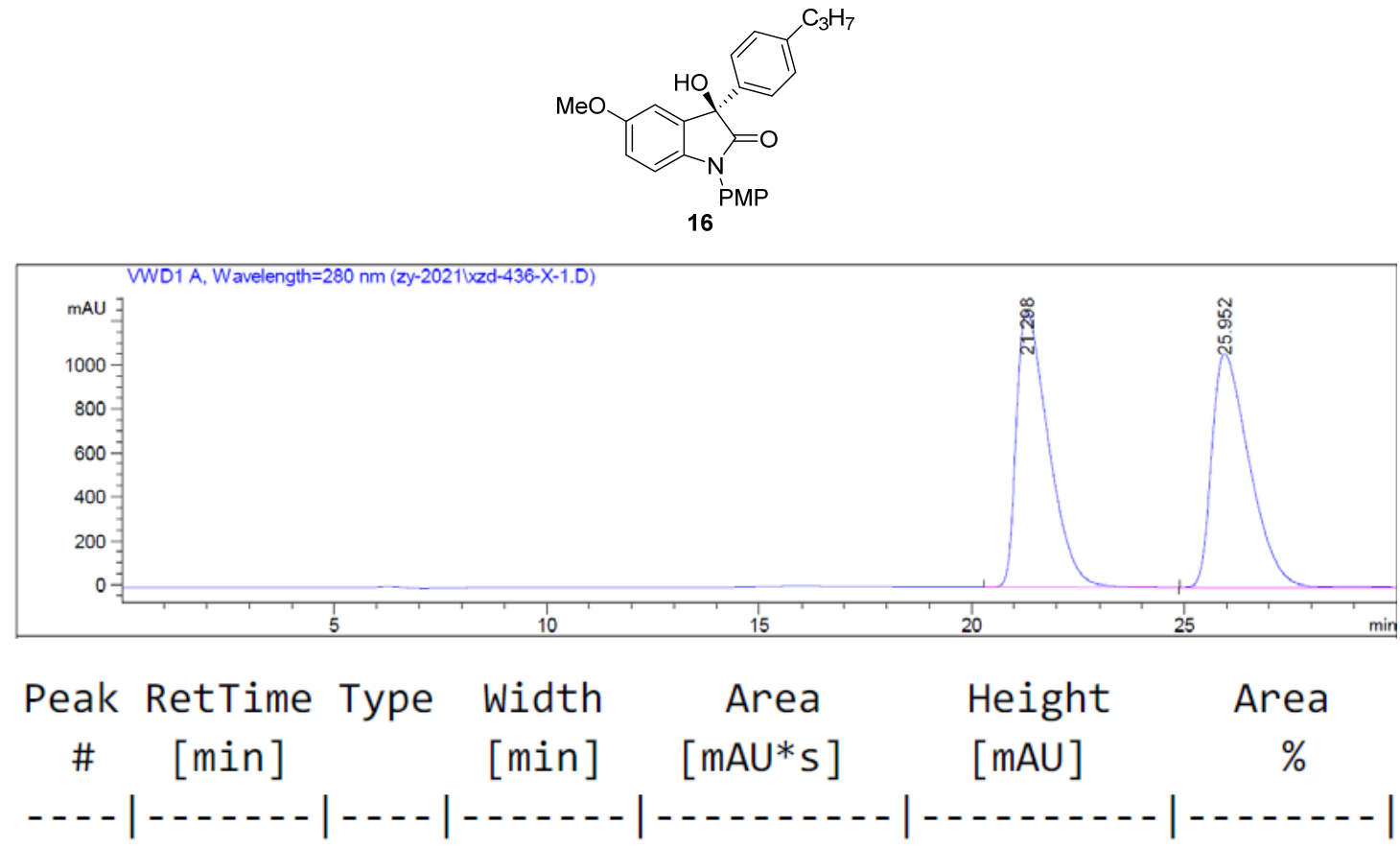

$\begin{array}{lllllll}1 & 21.298 \text { BB } & 0.8002 & 6.58207 \mathrm{e} 4 & 1254.31006 & 50.1825\end{array}$

$\begin{array}{lllllll}2 & 25.952 & \text { BV R } & 0.9388 & 6.53420 \mathrm{e} 4 & 1061.42981 & 49.8175\end{array}$

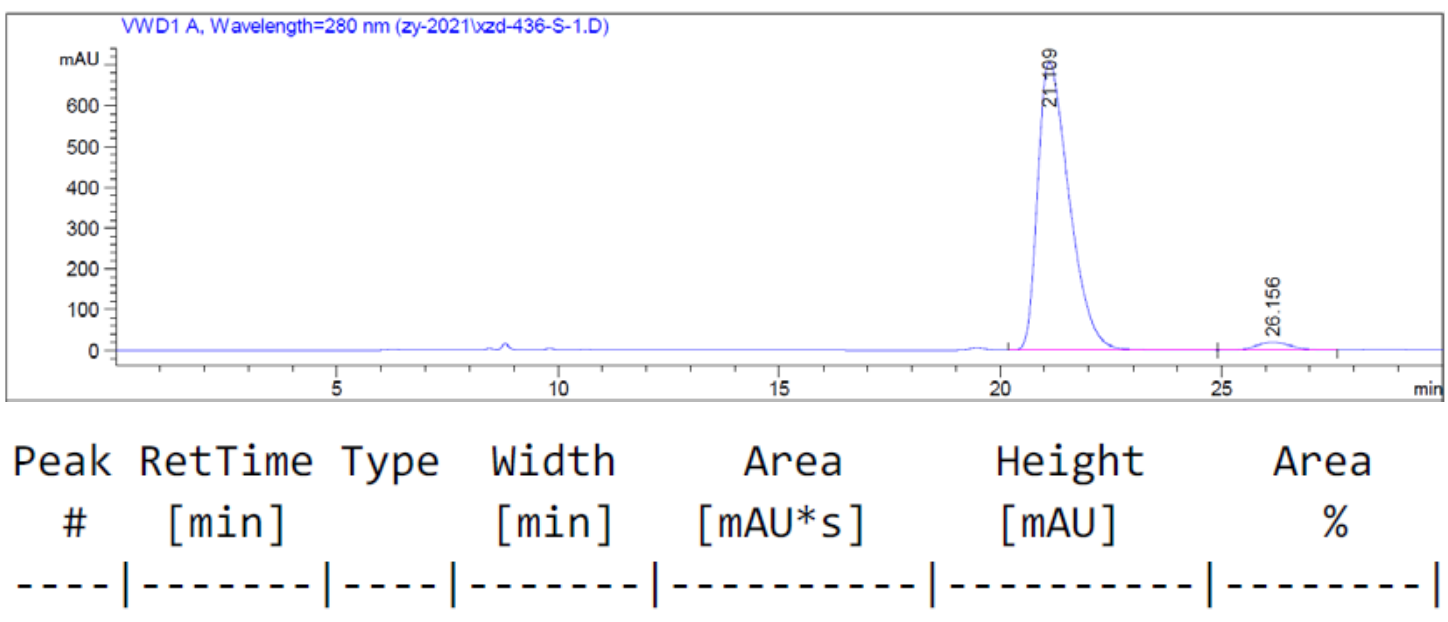

$\begin{array}{lllllll}1 & 21.109 & \text { BB } & 0.7636 & 3.50842 \mathrm{e} 4 & 706.26184 & 96.8992\end{array}$

$\begin{array}{lllllll}2 & 26.156 & \text { BB } & 0.8704 & 1122.69495 & 20.07806 & 3.1008\end{array}$ 
HPLC Spectra of 17.
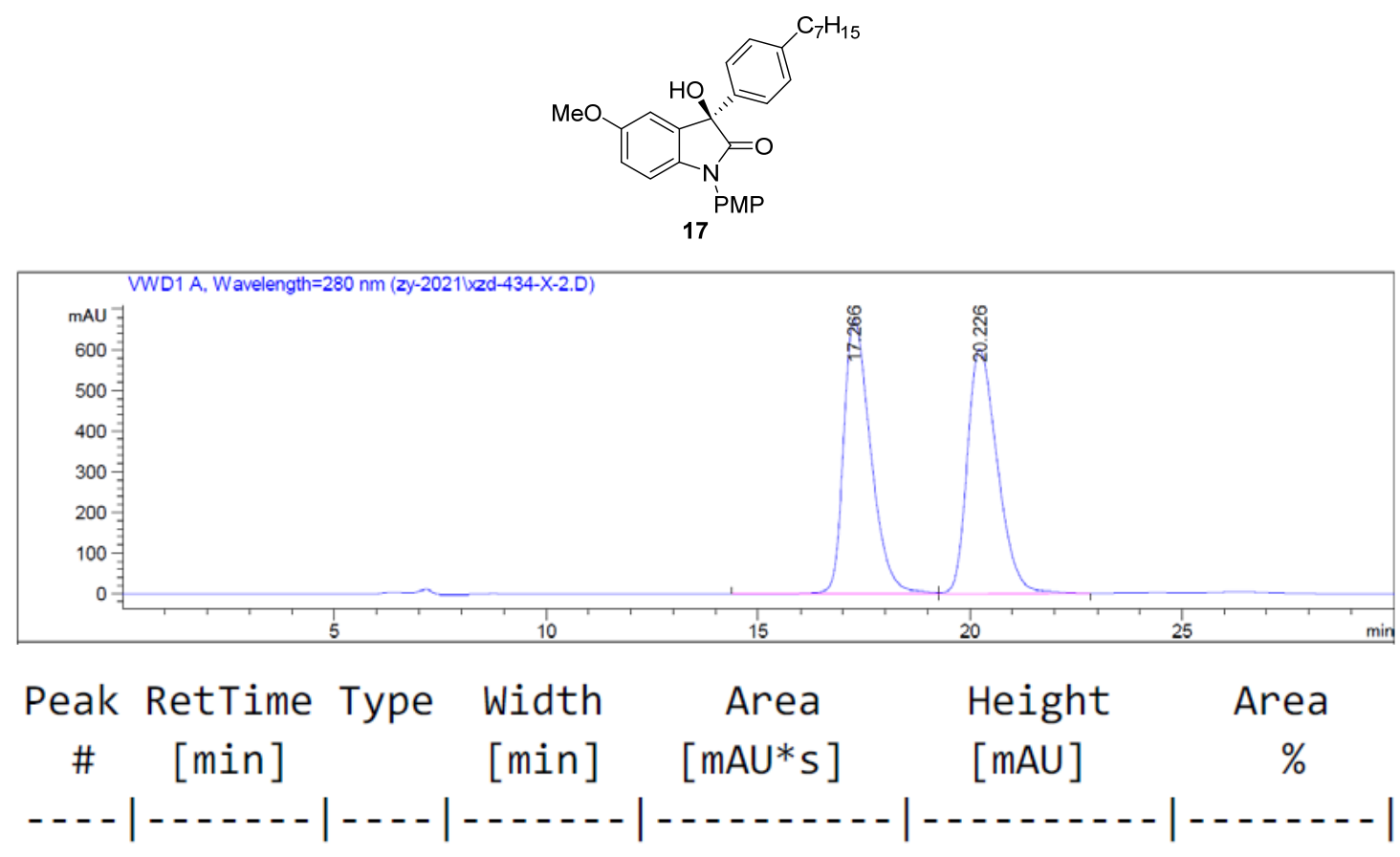

$\begin{array}{lllllll}1 & 17.266 & \text { BV } & 0.6806 & 3.00737 \mathrm{e} 4 & 676.51428 & 50.3495\end{array}$

$\begin{array}{lllllll}2 & 20.226 & \text { VB } & 0.7629 & 2.96561 \mathrm{e} 4 & 601.83826 & 49.6505\end{array}$

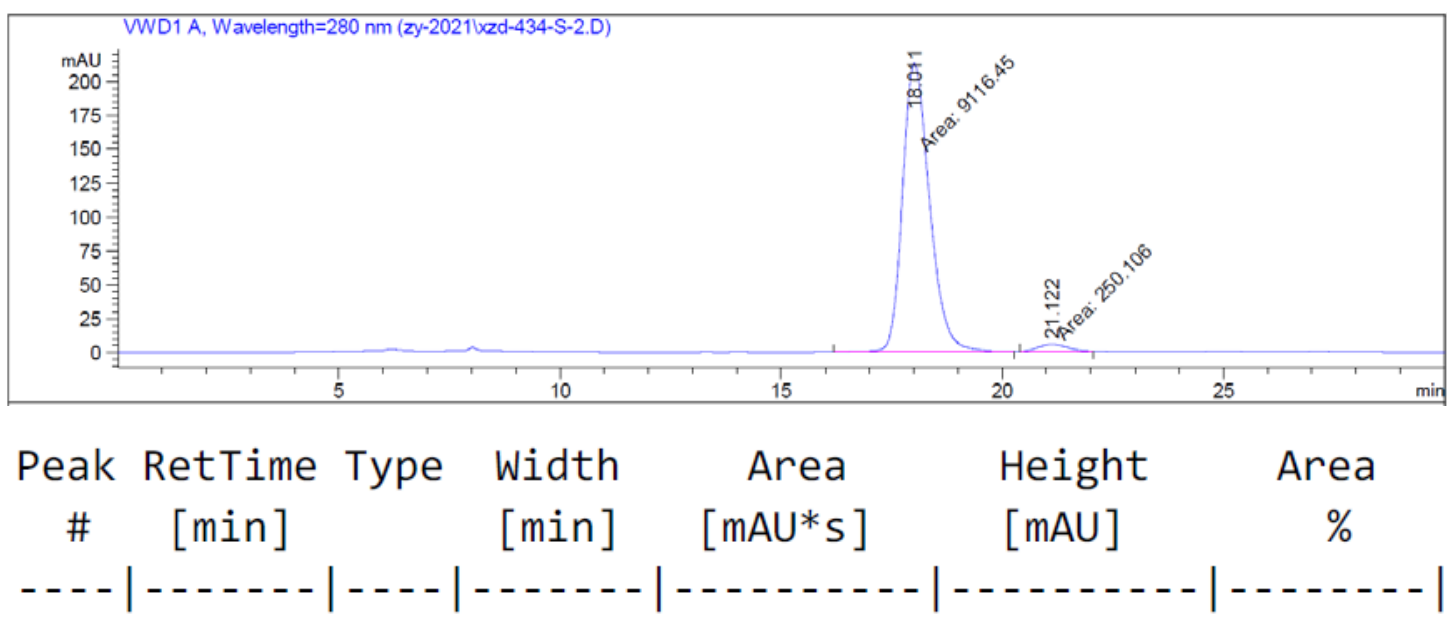

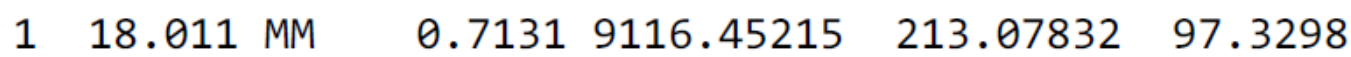

$\begin{array}{llllll}2 & 21.122 \text { MM } & 0.7566 & 250.10599 & 5.50969 & 2.6702\end{array}$ 
HPLC Spectra of $\mathbf{1 8 .}$
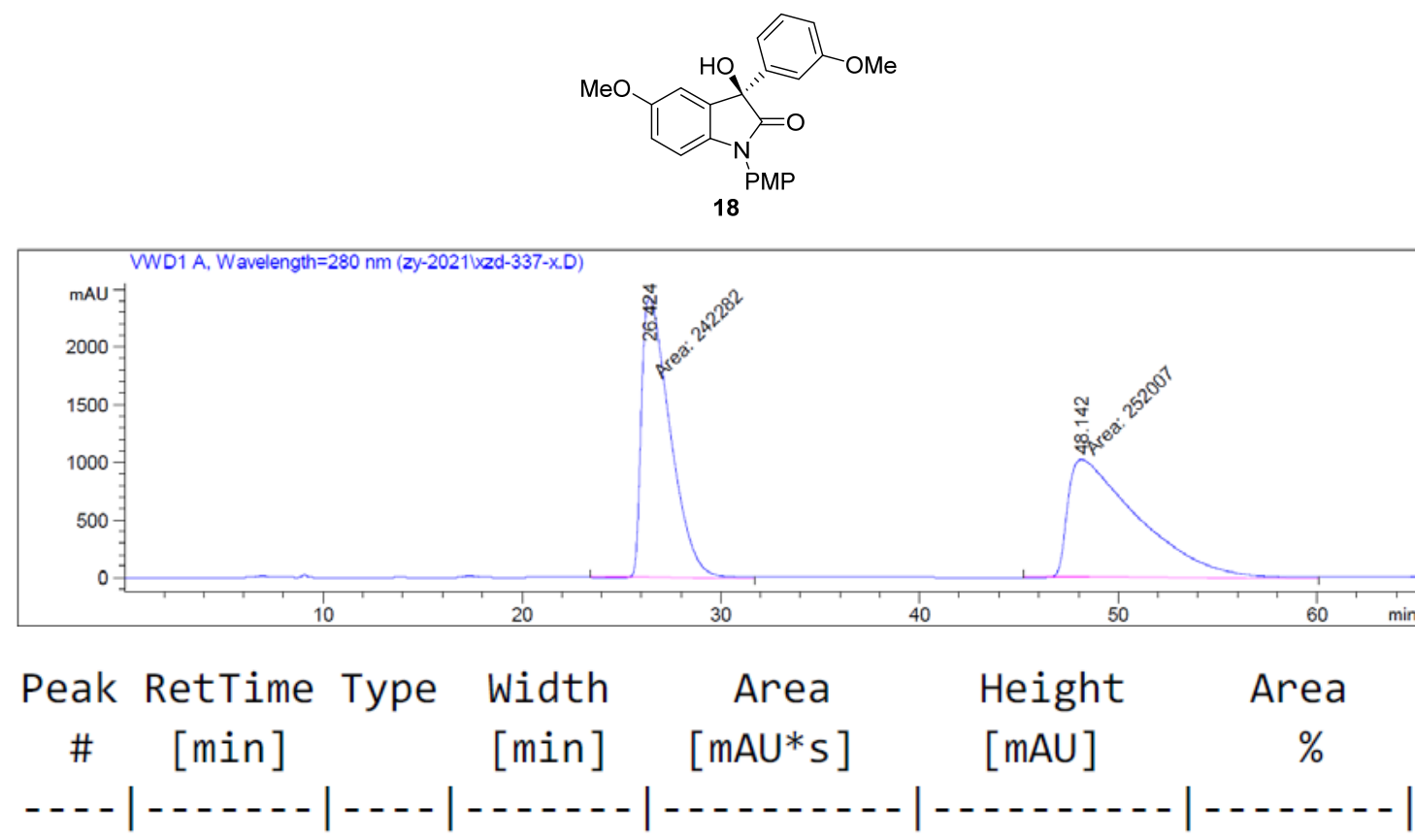

$\begin{array}{lllllll}1 & 26.424 \mathrm{MM} & 1.6616 & 2.42282 \mathrm{e} 5 & 2430.21191 & 49.0163\end{array}$

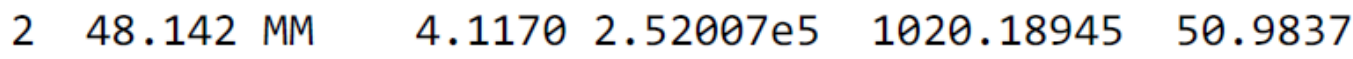

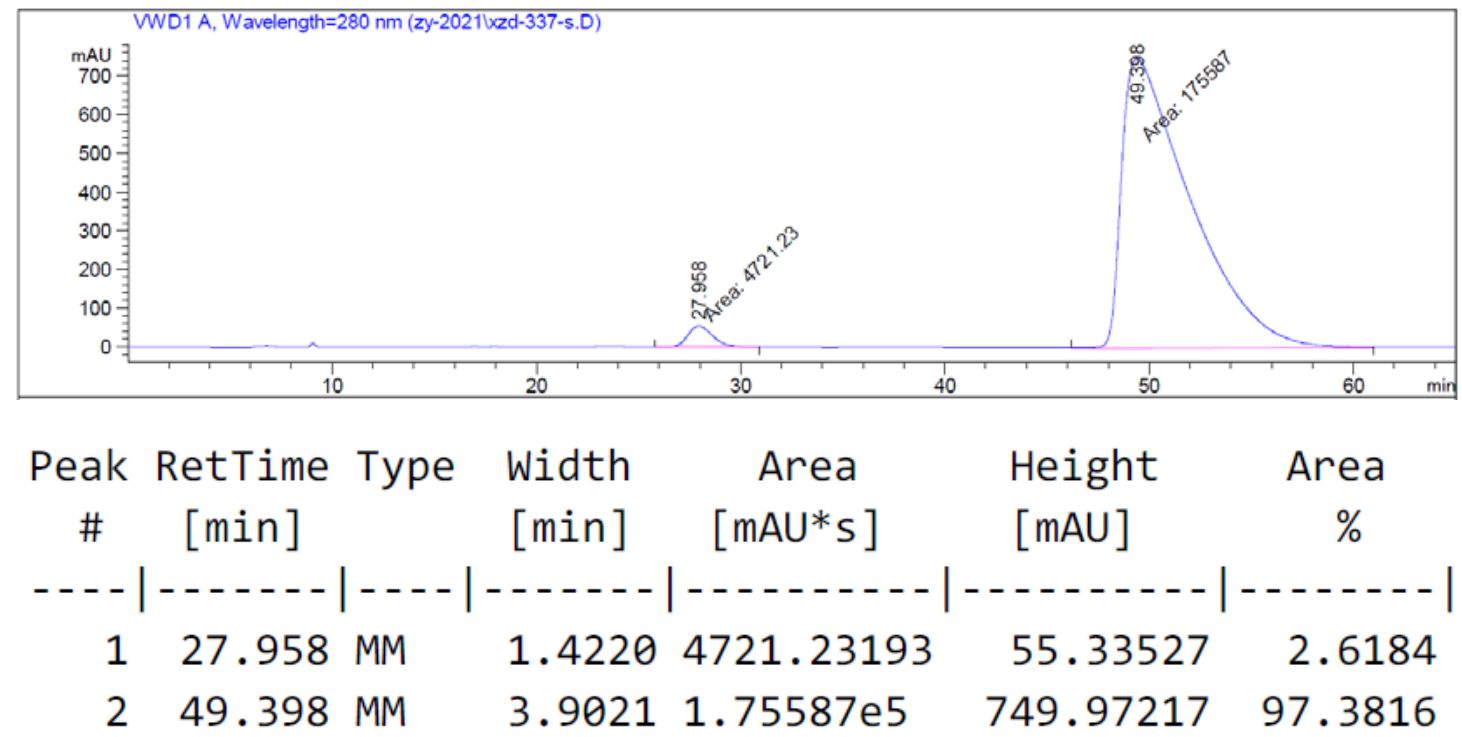


HPLC Spectra of 19.
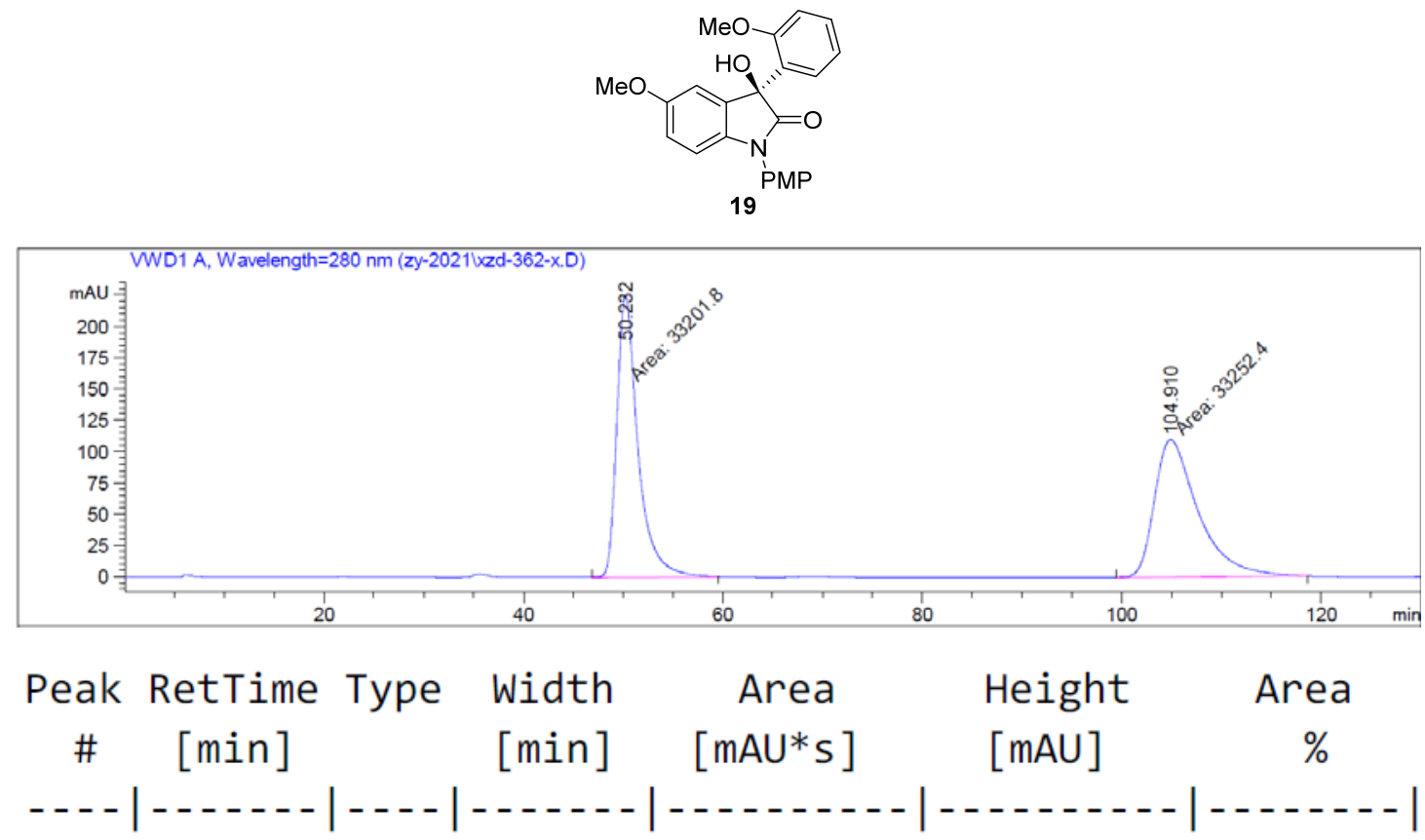

$\begin{array}{lllllll}1 & 50.232 & \text { MM } & 2.4613 & 3.32018 \mathrm{e} 4 & 224.82787 & 49.9619\end{array}$

$\begin{array}{llllll}2 & 104.910 \text { MM } & 5.0549 & 3.32524 \mathrm{e} 4 & 109.63779 & 50.0381\end{array}$

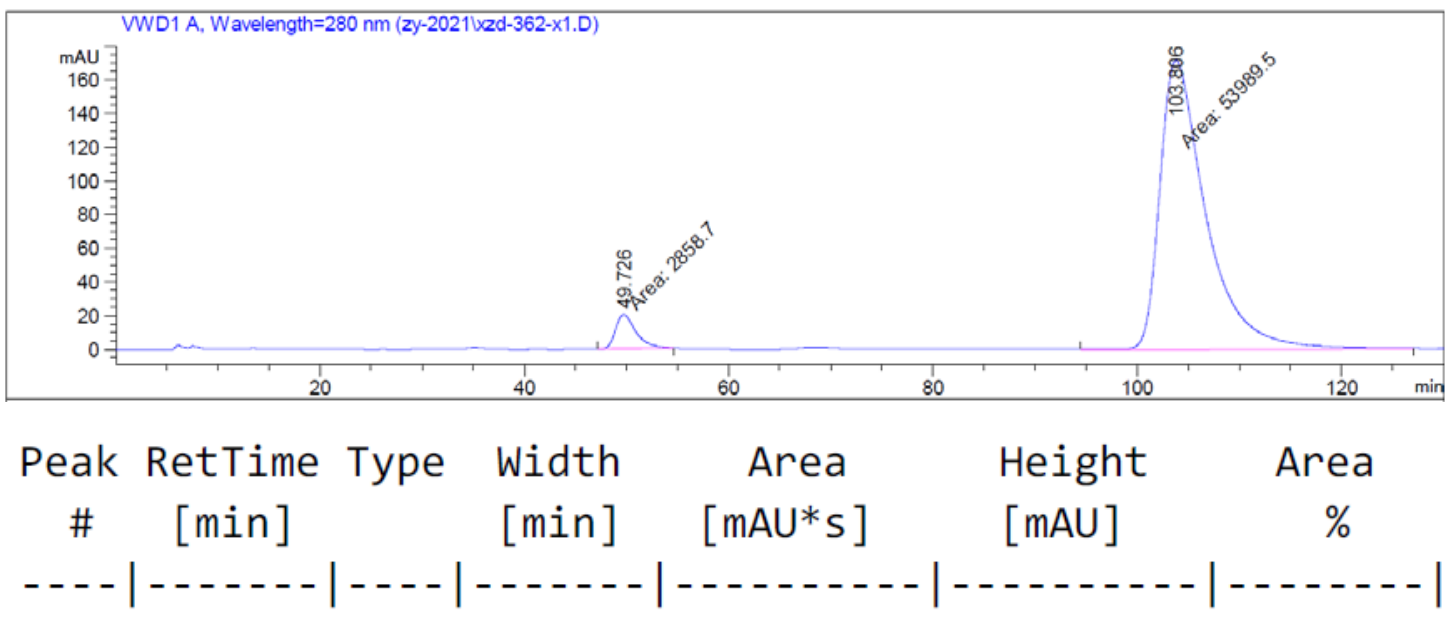

$\begin{array}{llllll}1 & 49.726 \text { MM } & 2.3259 & 2858.70313 & 20.48464 & 5.0287\end{array}$

$\begin{array}{llllll}2 & 103.806 \text { MM } & 5.2408 & 5.39895 \mathrm{e} 4 & 171.69510 & 94.9713\end{array}$ 
HPLC Spectra of $\mathbf{2 0 .}$
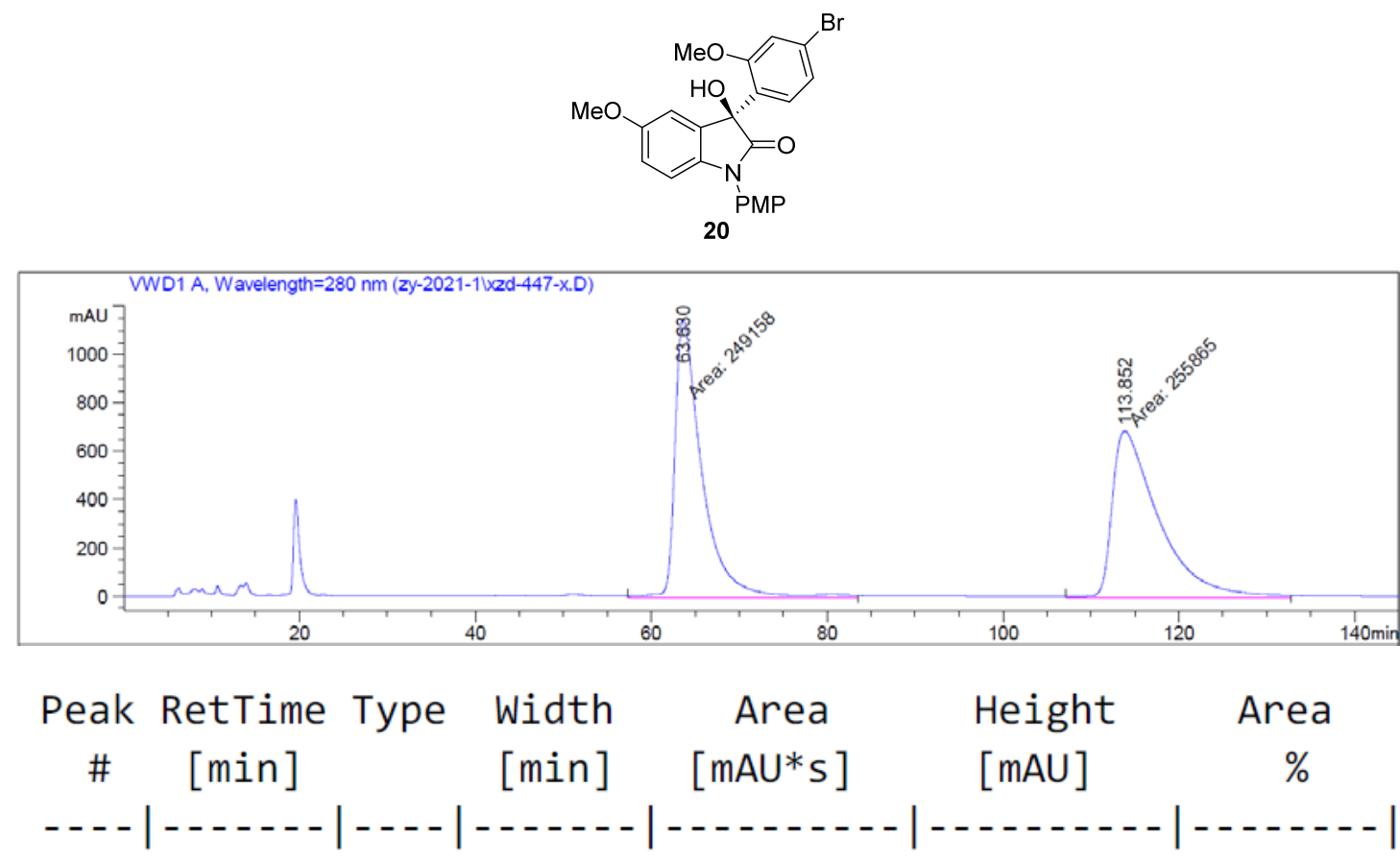

$\begin{array}{lllllll}1 & 63.630 & \text { MM } & 3.6004 & 2.49158 \mathrm{e} 5 & 1153.37695 & 49.3359\end{array}$

$\begin{array}{llllll}2 & 113.852 \text { MM } & 6.1745 & 2.55865 \mathrm{e} 5 & 690.64746 & 50.6641\end{array}$

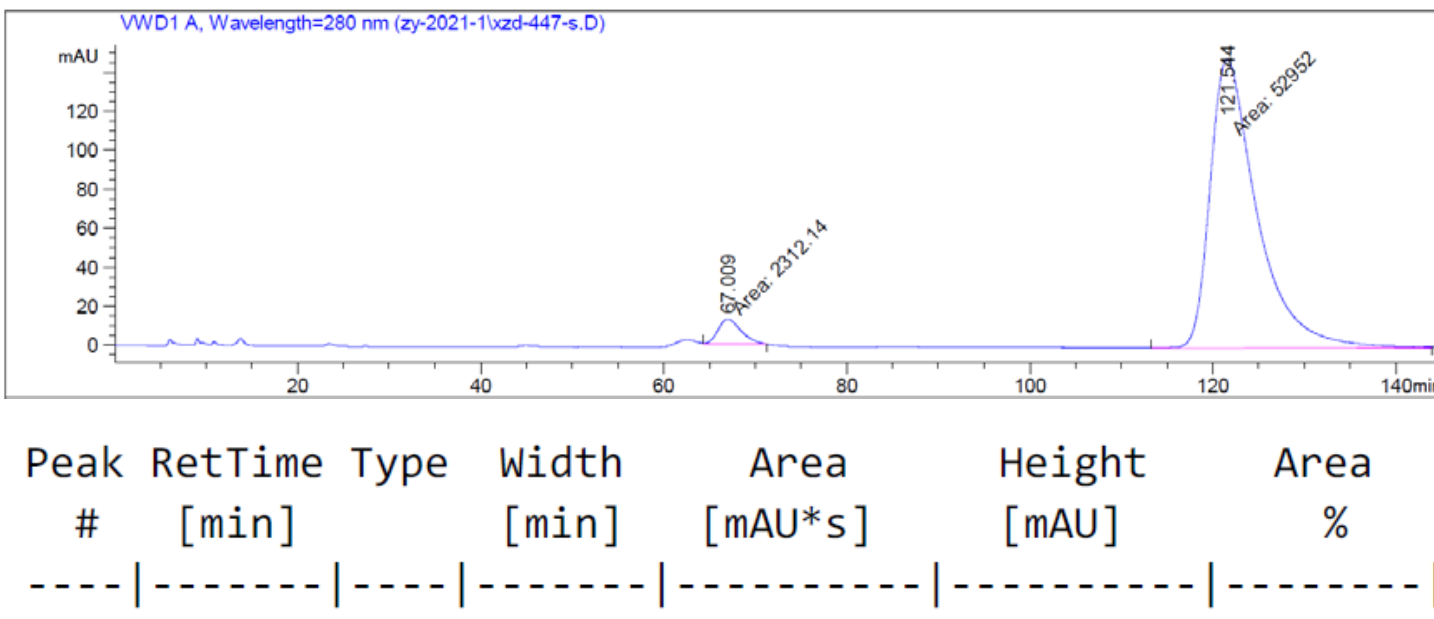

$\begin{array}{lllllll}1 & 67.009 & \text { MM } & 2.9676 & 2312.13989 & 12.98533 & 4.1838\end{array}$

$\begin{array}{llllll}2 & 121.544 \text { MM } & 5.9536 & 5.29520 \mathrm{e} 4 & 148.23419 & 95.8162\end{array}$ 
HPLC Spectra of 21.
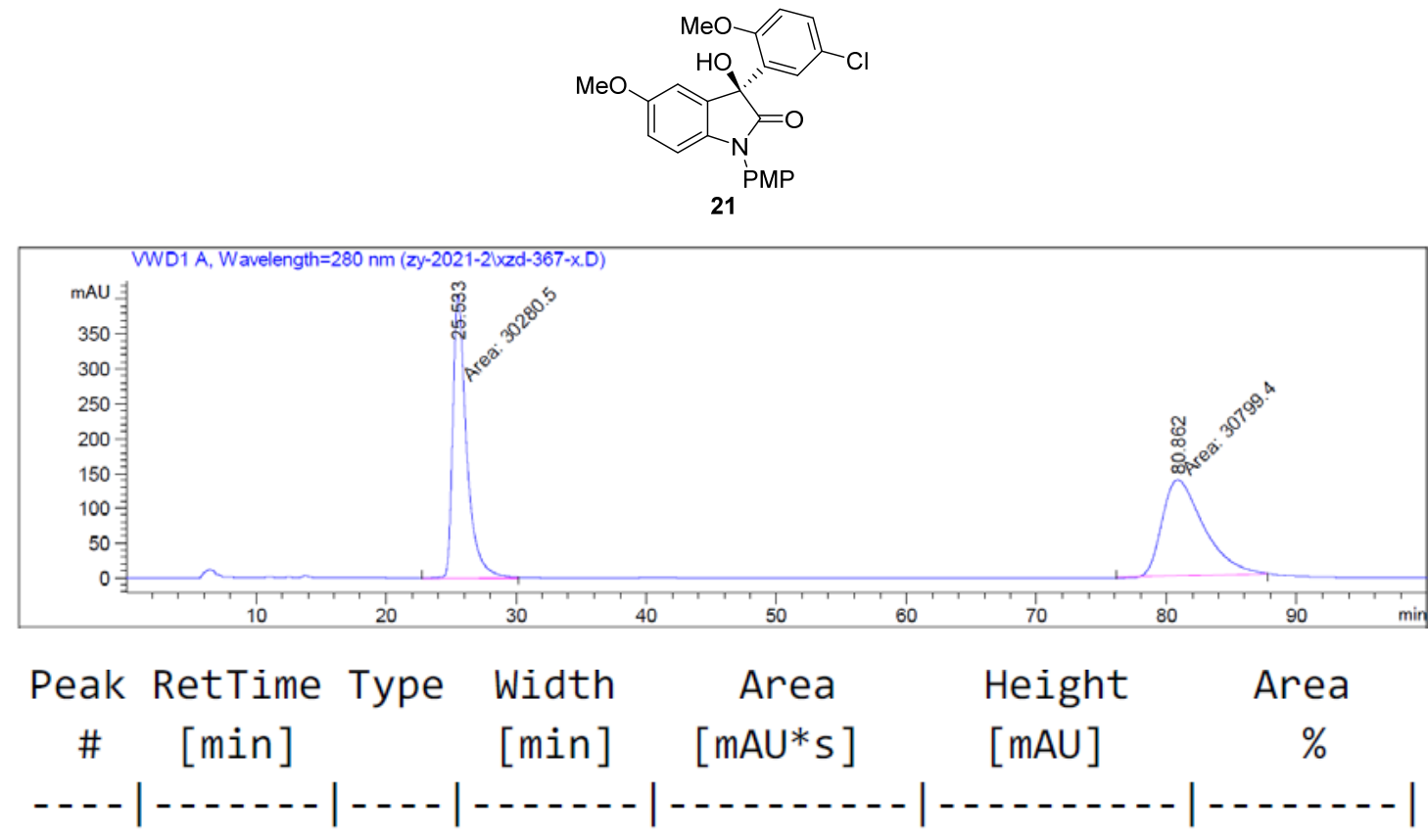

$\begin{array}{lllllll}1 & 25.533 \text { MM } & 1.2400 & 3.02805 \mathrm{e} 4 & 407.00168 & 49.5752\end{array}$

$\begin{array}{lllllll}2 & 80.862 \text { MM } \quad 3.7210 & 3.07994 \mathrm{e} & 137.95447 & 50.4248\end{array}$

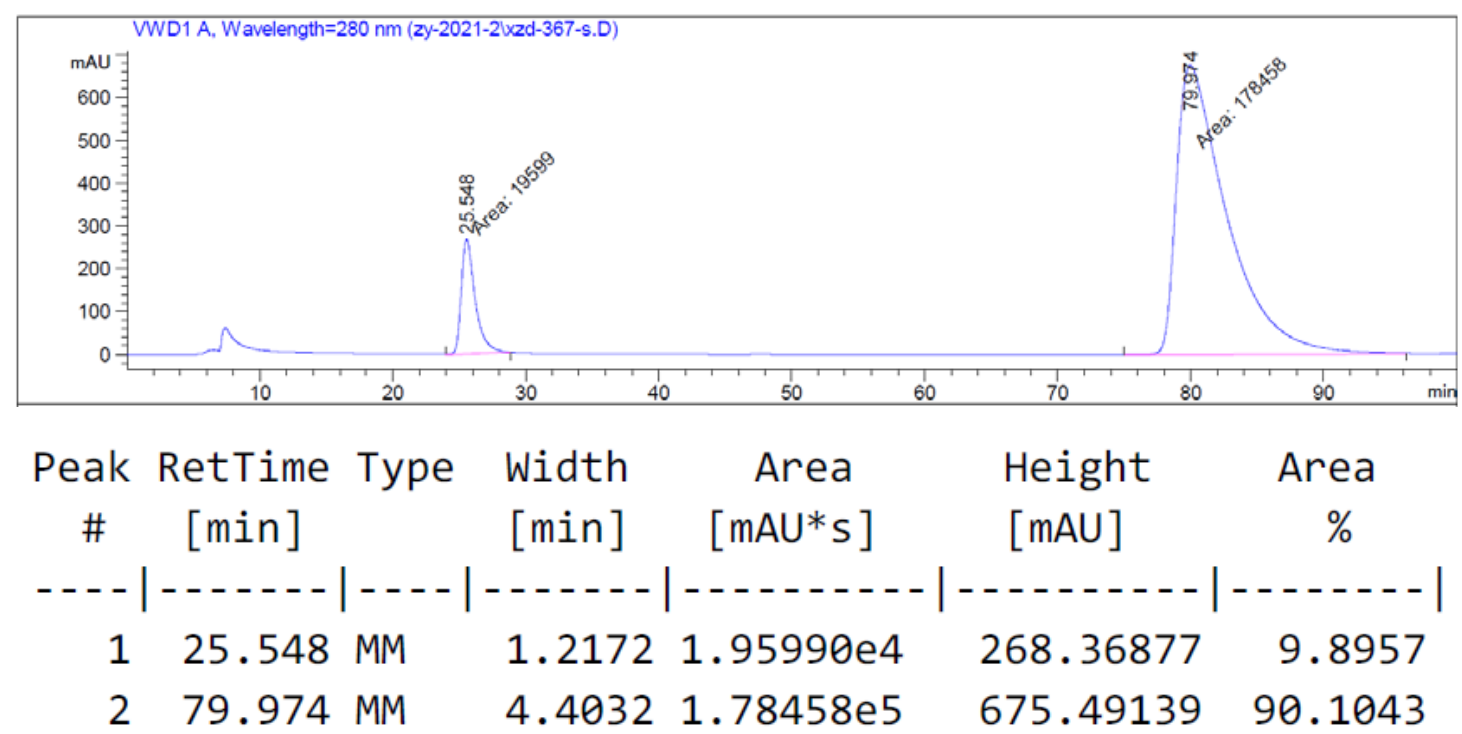


HPLC Spectra of 22.
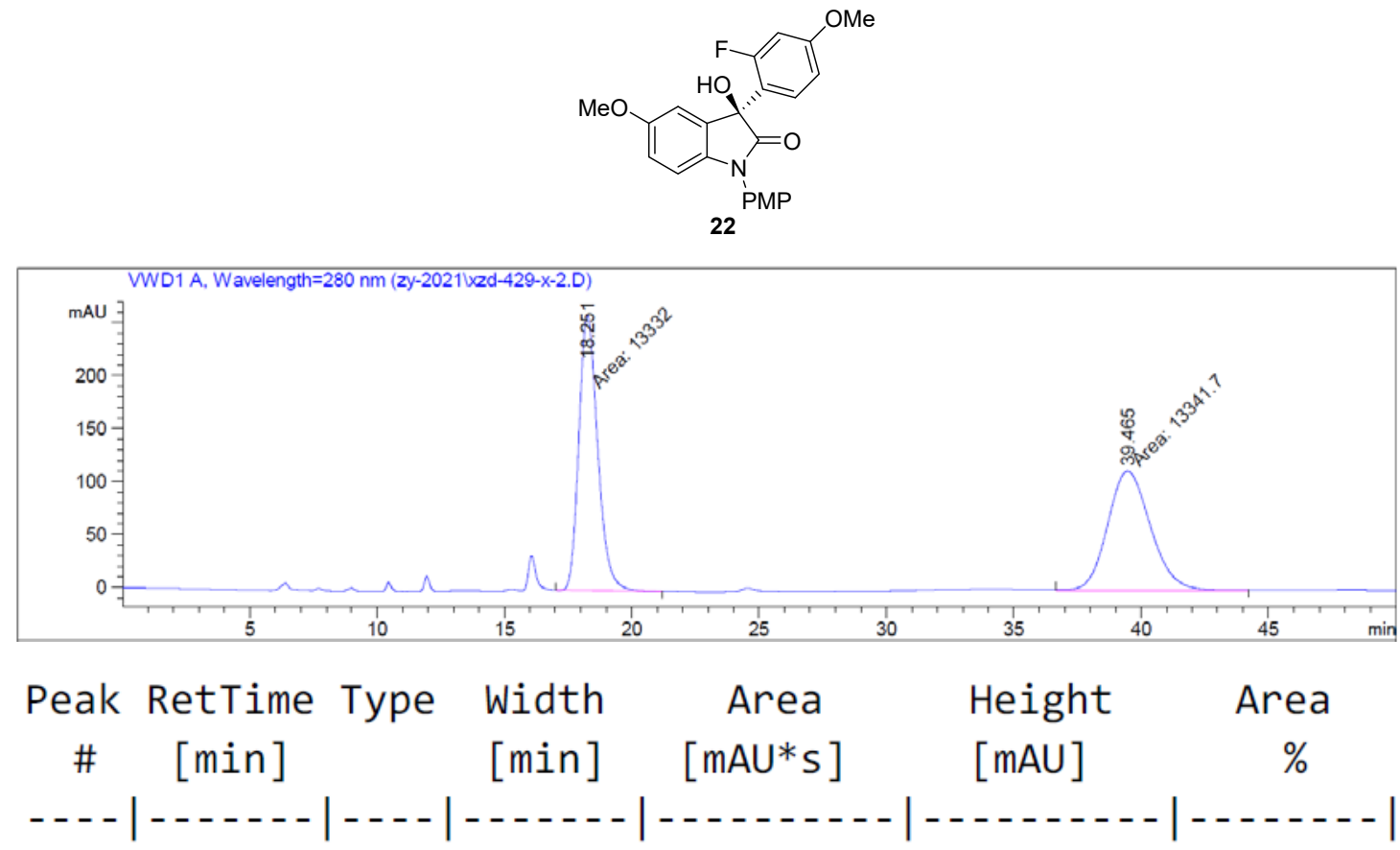

$\begin{array}{lllllll}1 & 18.251 & \text { MM } & 0.8540 & 1.33320 \mathrm{e} 4 & 260.18454 & 49.9819\end{array}$

$\begin{array}{llllll}2 & 39.465 \text { MM } & 1.9680 & 1.33417 \mathrm{e} 4 & 112.98778 & 50.0181\end{array}$

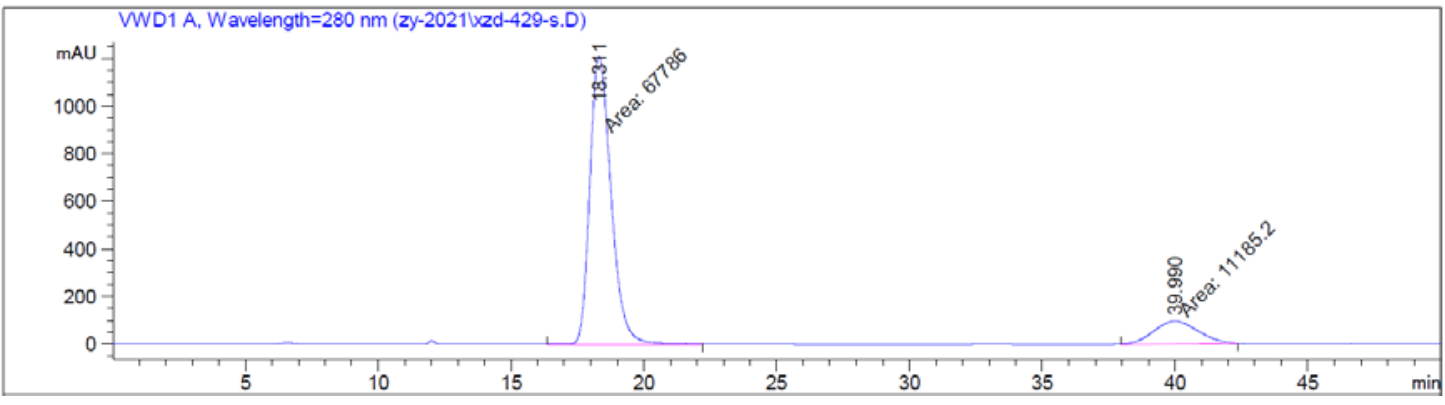

Peak RetTime Type Width Area Height Area

\# $[\mathrm{min}] \quad[\mathrm{min}] \quad[\mathrm{mAU} * \mathrm{~s}] \quad[\mathrm{mAU}] \quad \%$

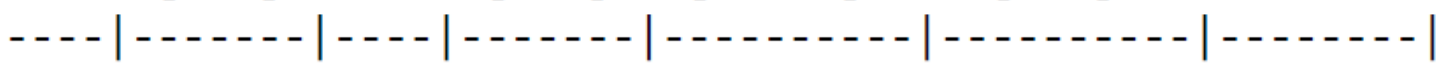

$\begin{array}{lllllll}1 & 18.311 & \text { MM } & 0.9328 & 6.77860 \mathrm{e} 4 & 1211.11072 & 85.8363\end{array}$

$\begin{array}{llllll}2 & 39.990 \text { MM } & 1.9376 & 1.11852 \mathrm{e} 4 & 96.21155 & 14.1637\end{array}$ 
HPLC Spectra of 23.
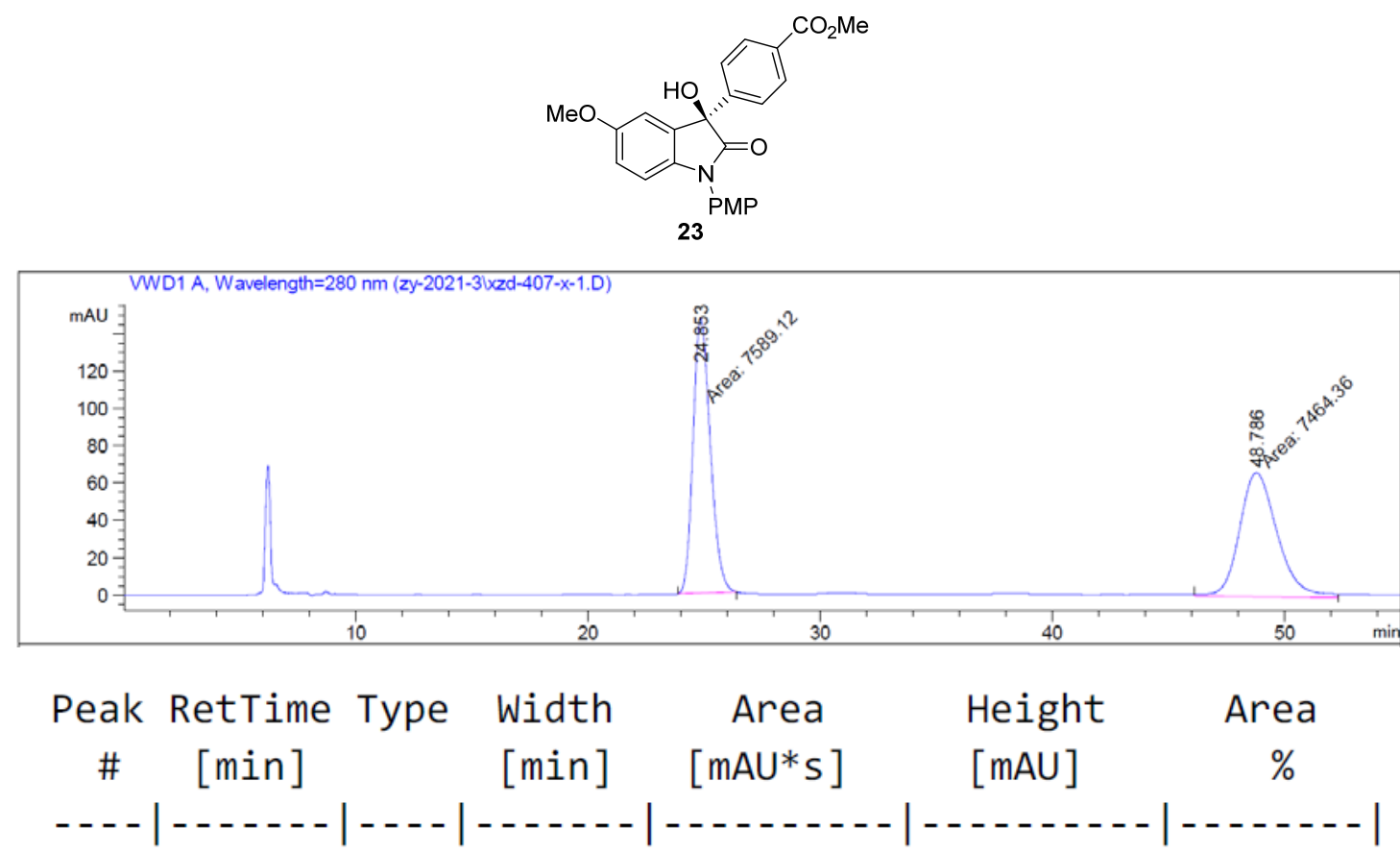

$\begin{array}{lllllll}1 & 24.853 \text { MM } & 0.8599 & 7589.11768 & 147.09985 & 50.4144\end{array}$

$\begin{array}{lllllll}2 & 48.786 \text { MM } & 1.8773 & 7464.35791 & 66.26744 & 49.5856\end{array}$

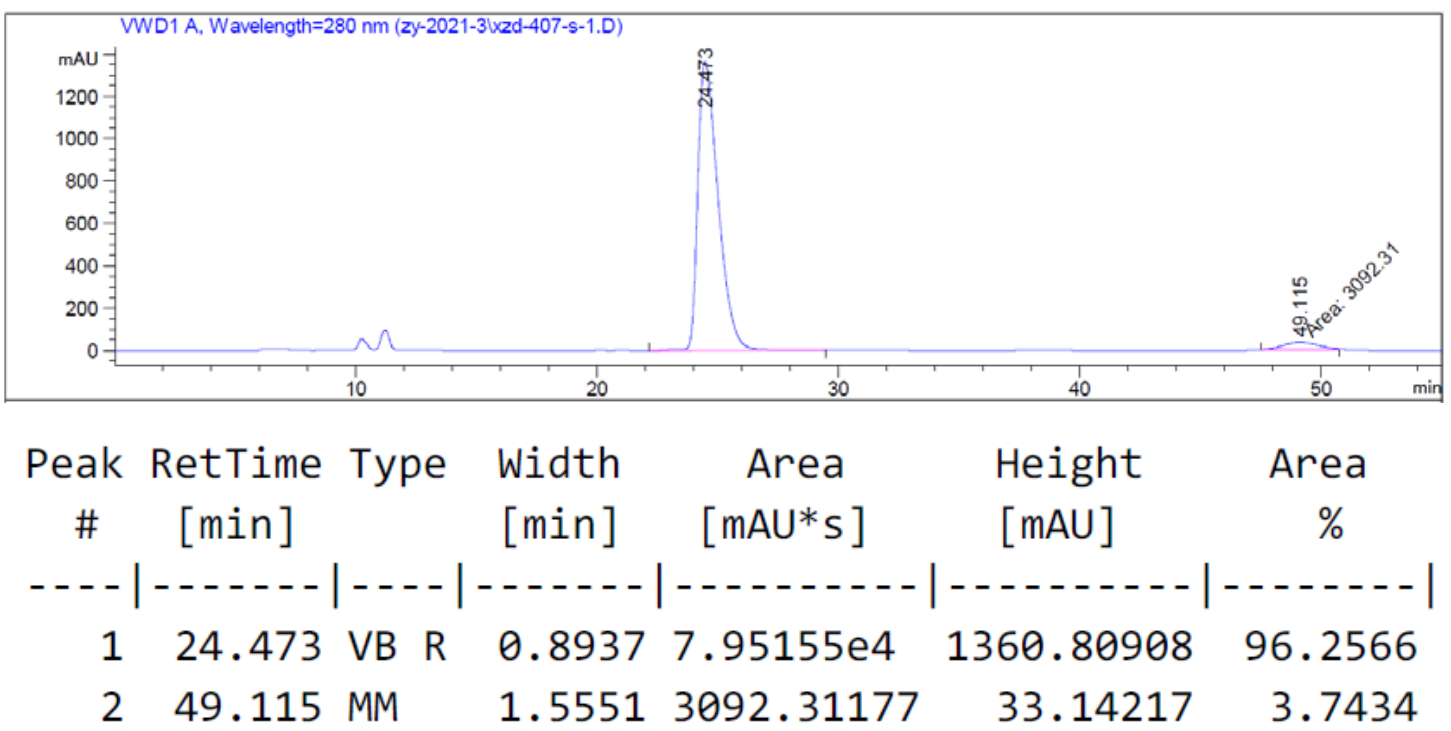


HPLC Spectra of 24.
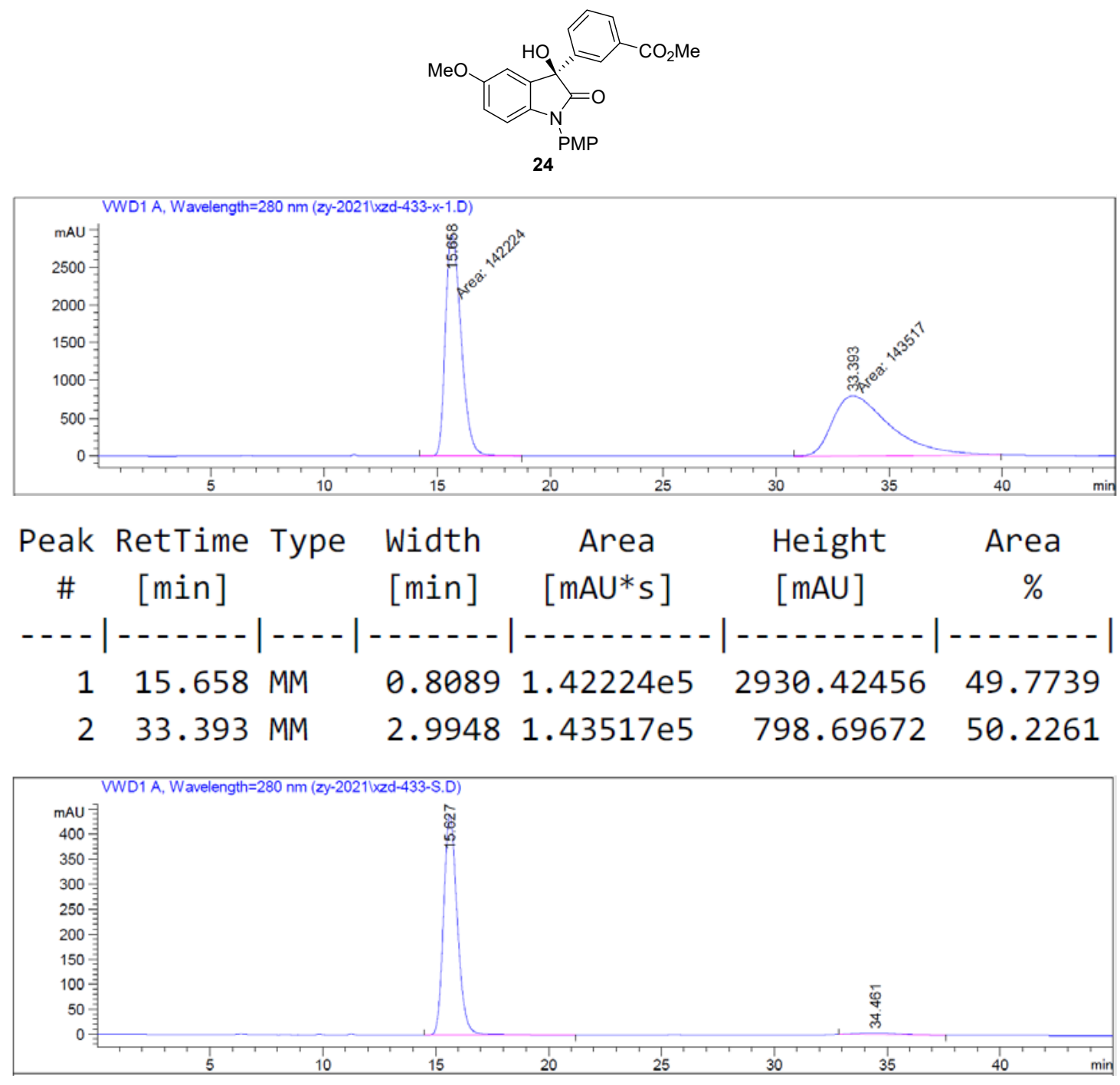

\begin{tabular}{|c|c|c|c|c|c|}
\hline $\begin{array}{c}\text { Peak } \\
\text { A }\end{array}$ & $\begin{array}{l}\text { RetTime Type } \\
\text { [min] }\end{array}$ & $\begin{array}{l}\text { Width } \\
\text { [min] }\end{array}$ & $\begin{array}{c}\text { Area } \\
{[\mathrm{mAU} * \mathrm{~s}]}\end{array}$ & $\begin{array}{l}\text { Height } \\
{[\mathrm{mAU}]}\end{array}$ & $\begin{array}{c}\text { Area } \\
\%\end{array}$ \\
\hline & & & & & \\
\hline 1 & 15.627 BB & 8 & $1.87182 \mathrm{e} 4$ & 440.42169 & 97.5758 \\
\hline 2 & $61 \mathrm{BB}$ & 60 & 465.03943 & 3.76771 & 2.4242 \\
\hline
\end{tabular}


HPLC Spectra of $\mathbf{2 5}$.
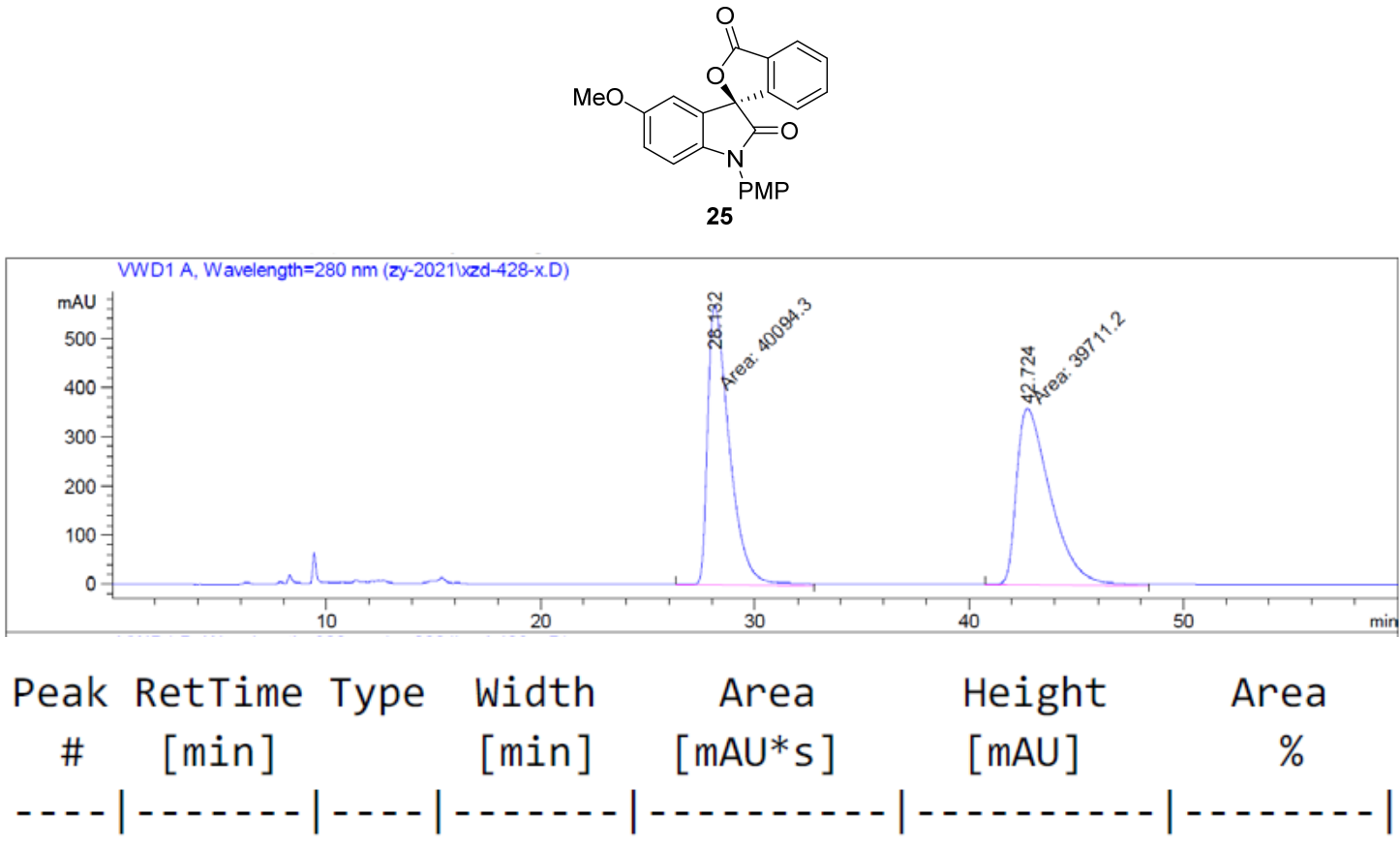

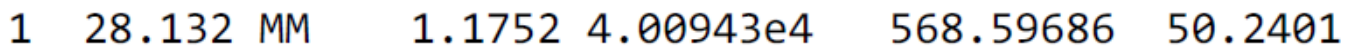

$\begin{array}{llllll}2 & 42.724 \text { MM } \quad 1.8479 & 3.97112 \mathrm{e} 4 & 358.16000 & 49.7599\end{array}$

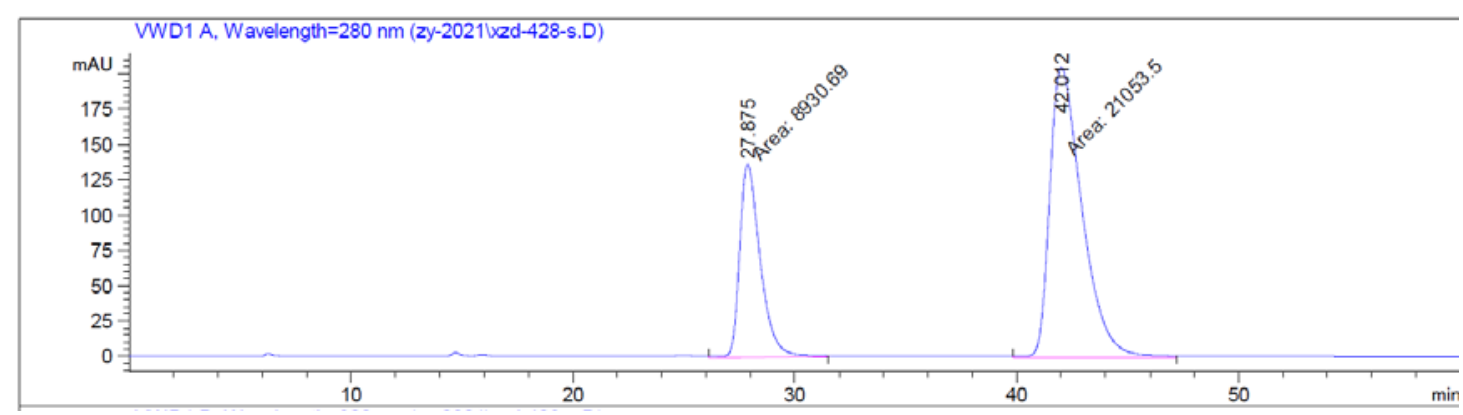

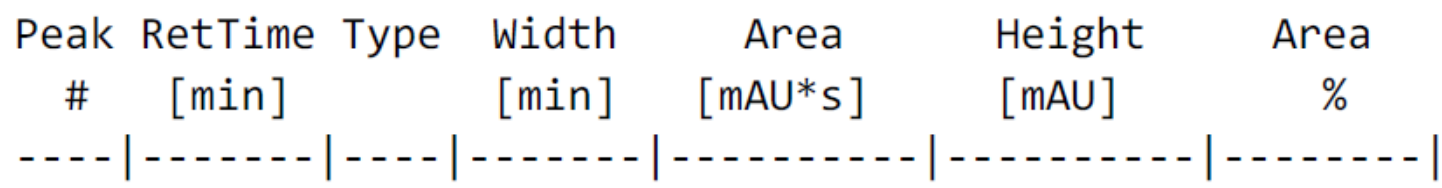

$\begin{array}{lllllll}1 & 27.875 & \text { MM } & 1.0868 & 8930.69238 & 136.96239 & 29.7847\end{array}$

$\begin{array}{llllll}2 & 42.012 \mathrm{MM} & 1.7034 & 2.10535 \mathrm{e} 4 & 205.99652 & 70.2153\end{array}$ 
HPLC Spectra of 26.
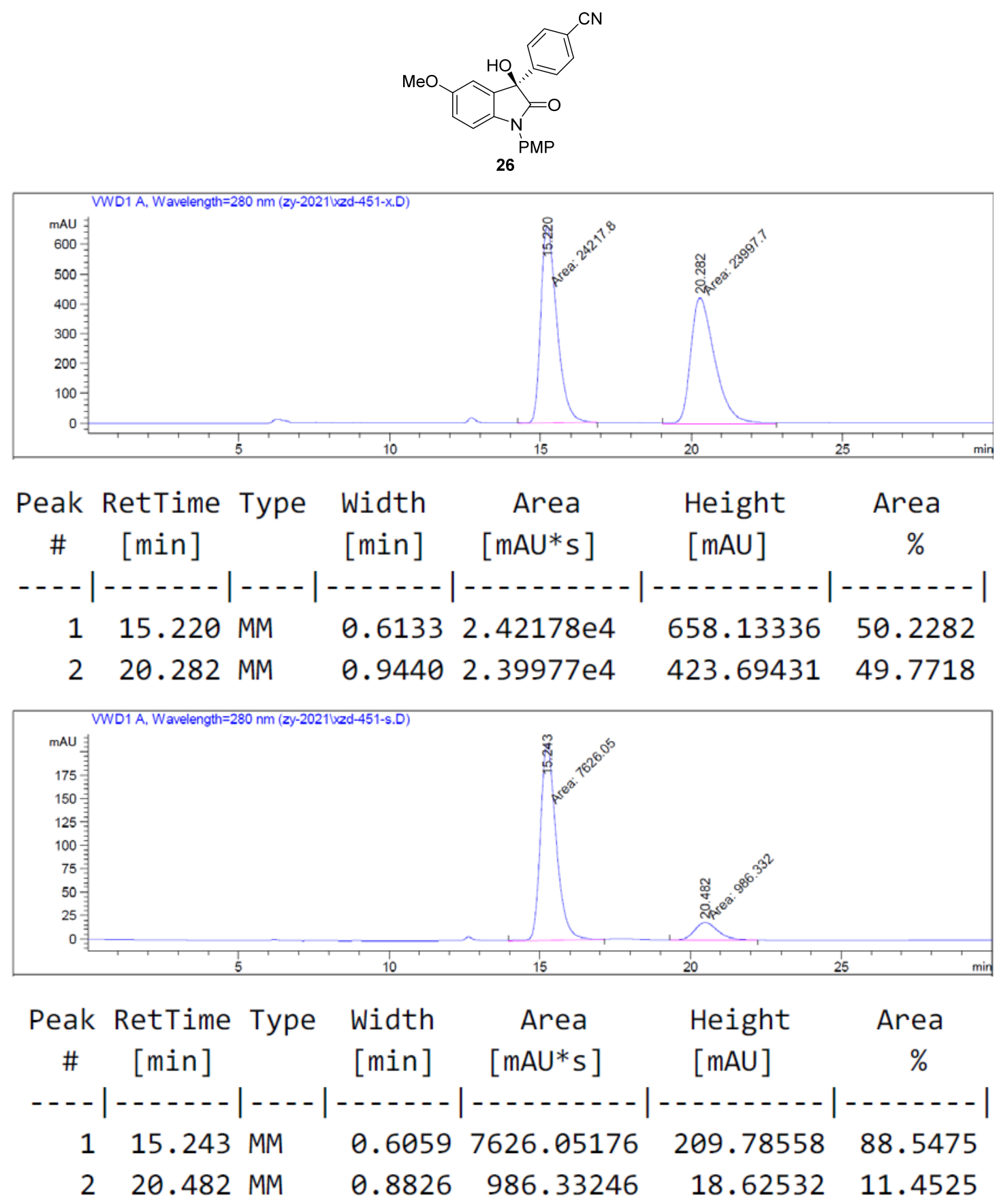
HPLC Spectra of 27.
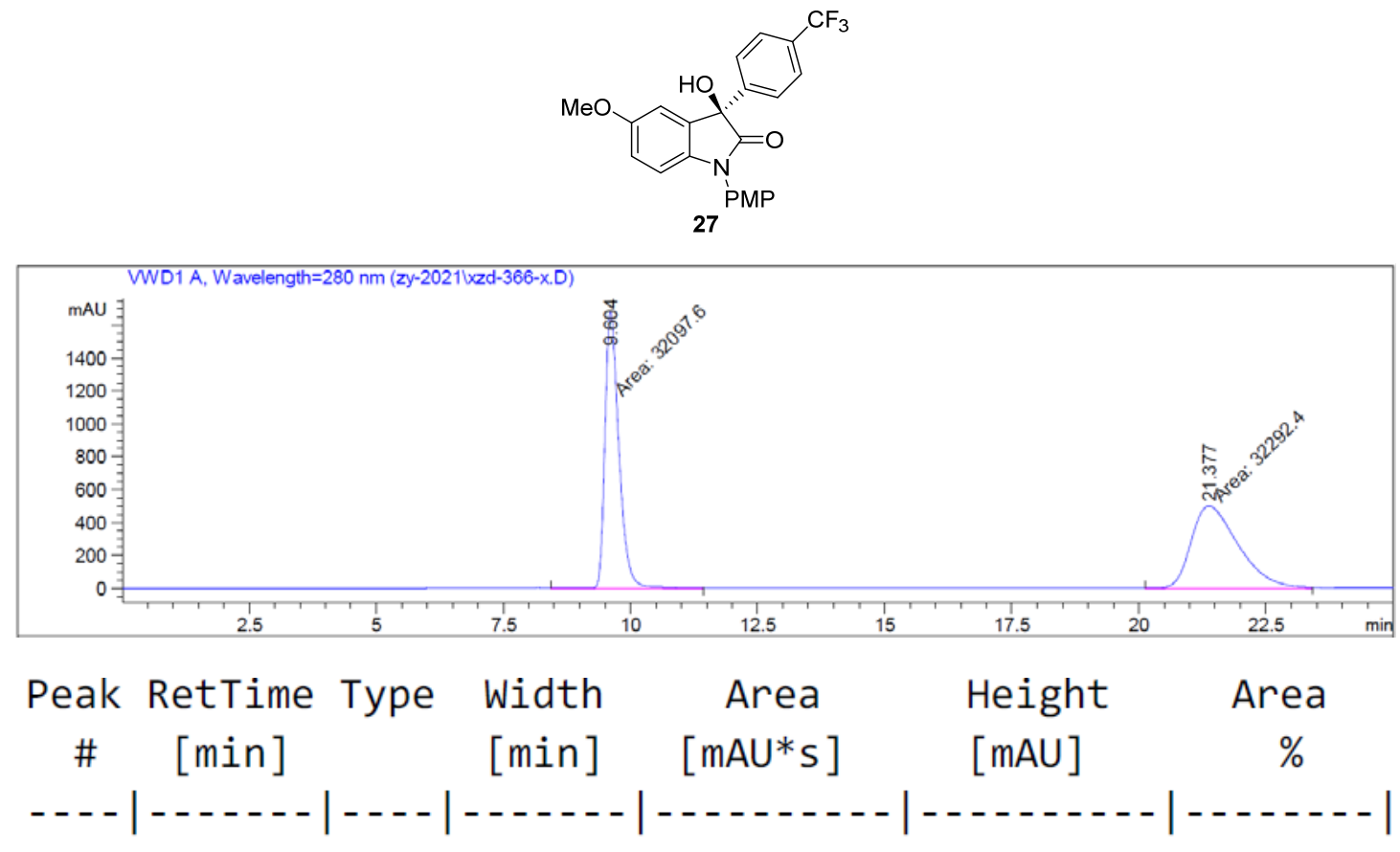

$\begin{array}{lllllll}1 & 9.604 \mathrm{MM} & 0.3189 & 3.20976 \mathrm{e} 4 & 1677.39929 & 49.8488\end{array}$

$\begin{array}{lllllll}2 & 21.377 \text { MM } & 1.0760 & 3.22924 \mathrm{e} 4 & 500.16907 & 50.1512\end{array}$
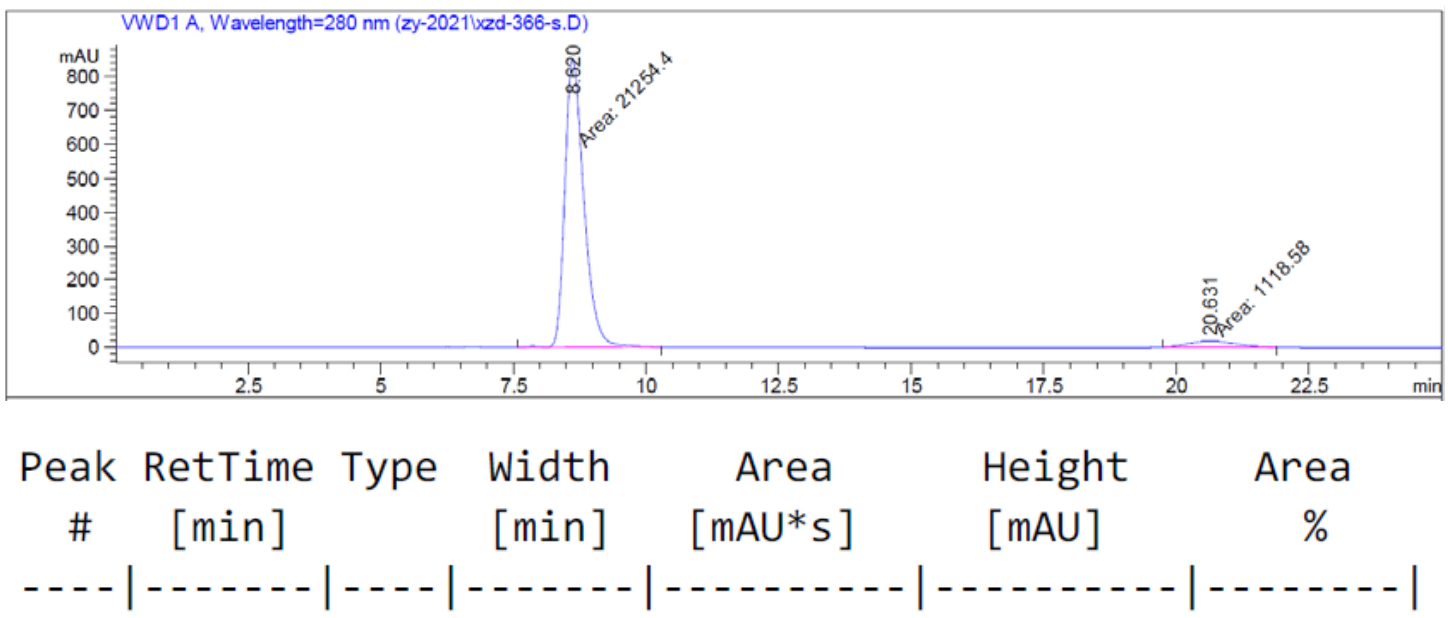
$18.620 \mathrm{MM}$
$0.4163 \quad 2.12544 \mathrm{e} 4 \quad 850.90790$
95.0003
$2 \quad 20.631 \mathrm{MM}$
$\begin{array}{lll}1.0117 & 1118.58252 \quad 18.42702\end{array}$
4.9997 
HPLC Spectra of $\mathbf{2 8}$
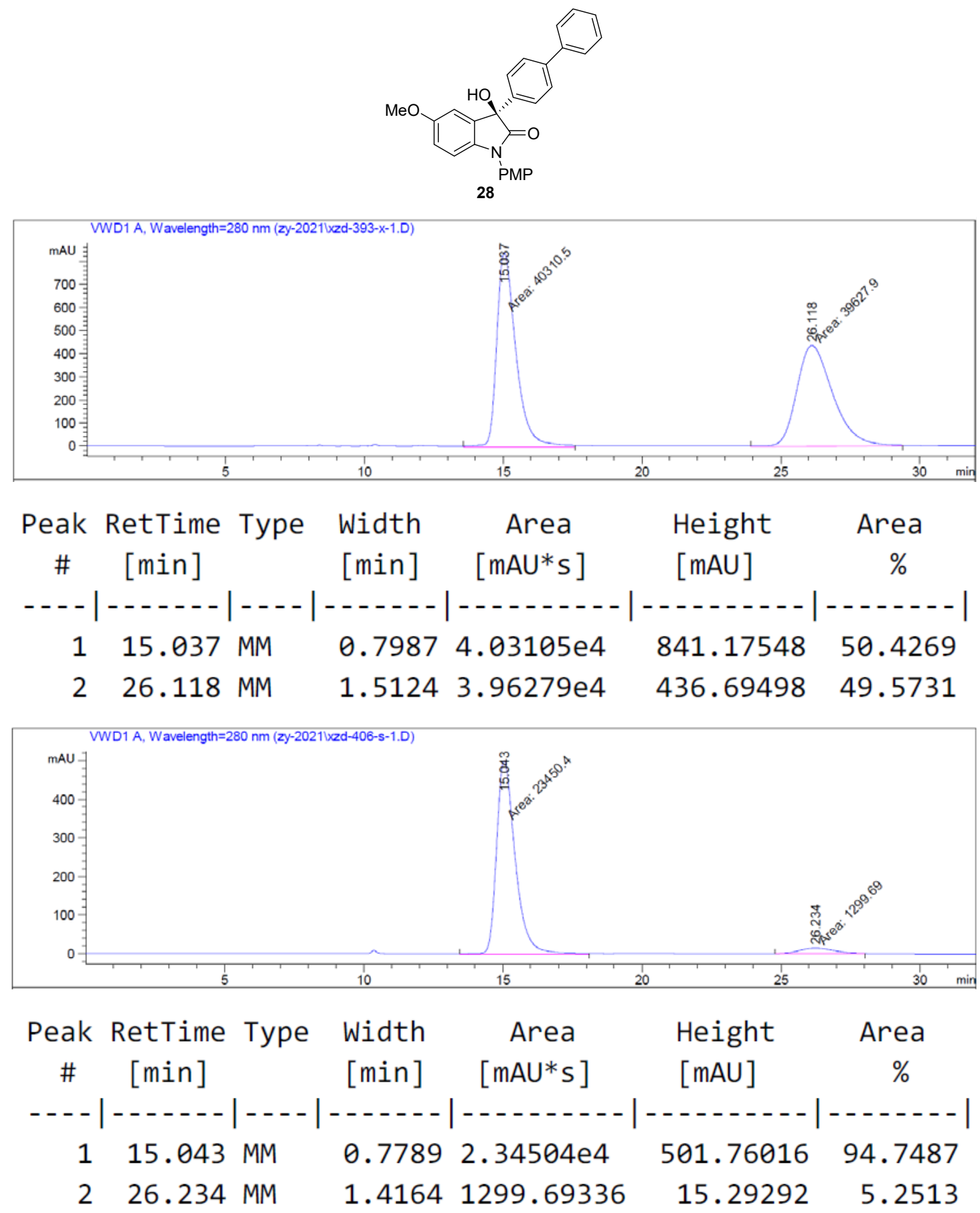
HPLC Spectra of 29.
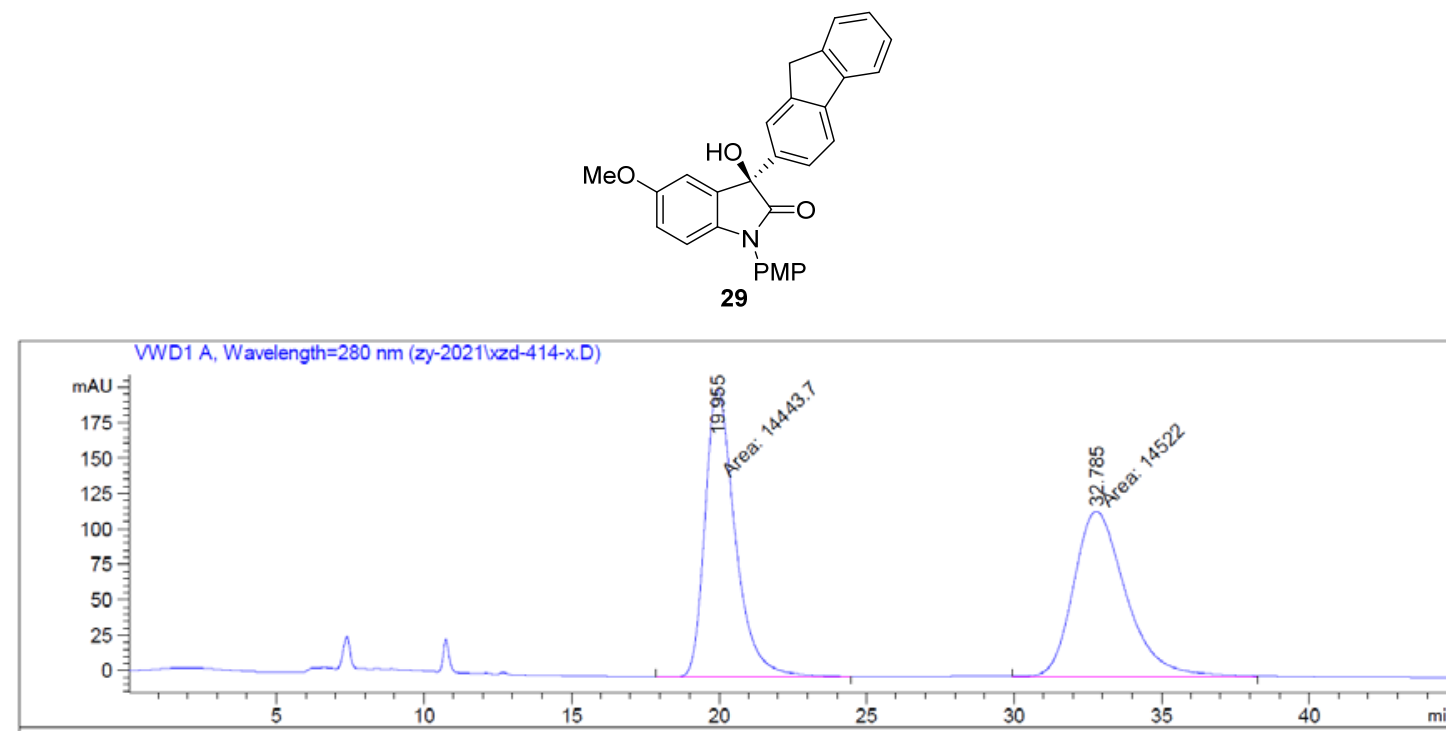

Peak RetTime Type Width Area Height Area

\# $\quad[\mathrm{min}] \quad[\mathrm{min}] \quad[\mathrm{mAU} * \mathrm{~s}] \quad[\mathrm{mAU}] \quad \%$

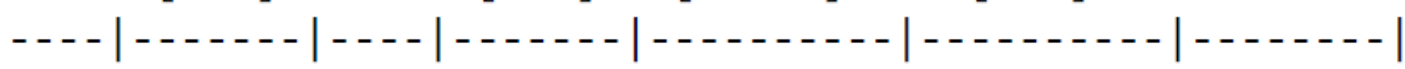

$\begin{array}{lllllll}1 & 19.955 & \text { MM } & 1.1867 & 1.44437 \mathrm{e} 4 & 202.86287 & 49.8648\end{array}$

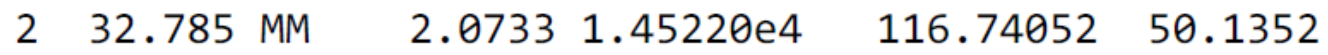

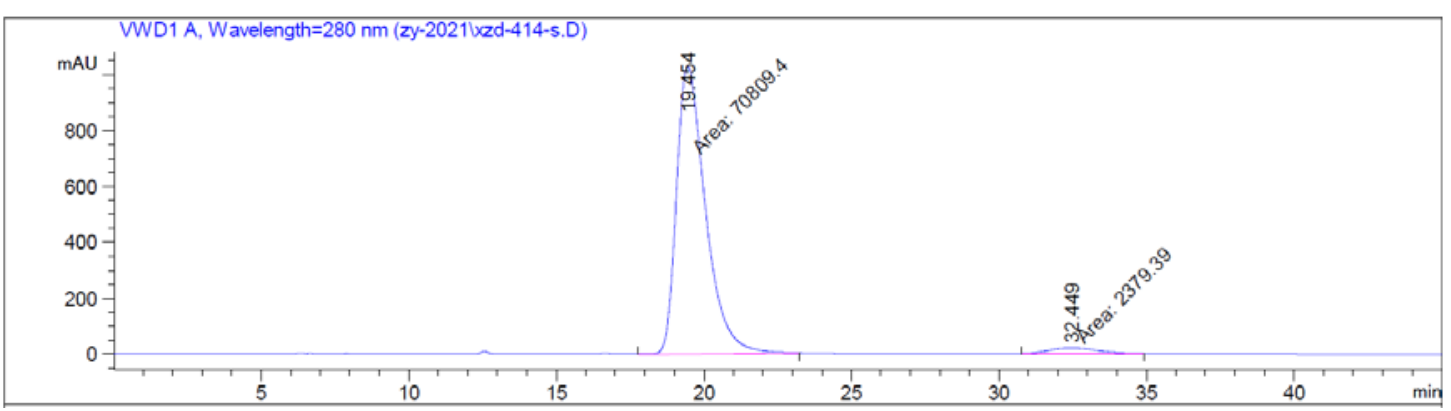

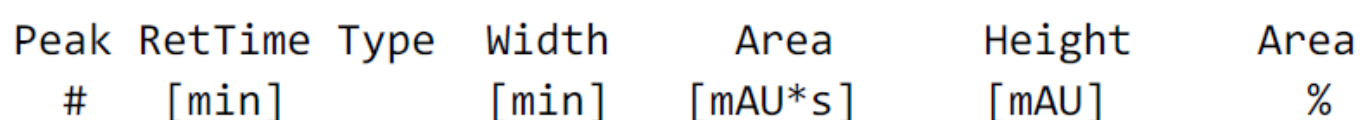

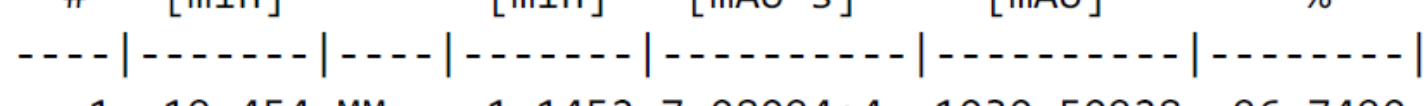

$\begin{array}{lllllll}1 & 19.454 \text { MM } & 1.1452 & 7.08094 \mathrm{e} 4 & 1030.50928 & 96.7490\end{array}$

$\begin{array}{llllll}2 & 32.449 \text { MM } & 1.8798 & 2379.38574 & 21.09661 & 3.2510\end{array}$ 
HPLC Spectra of $\mathbf{3 0}$.
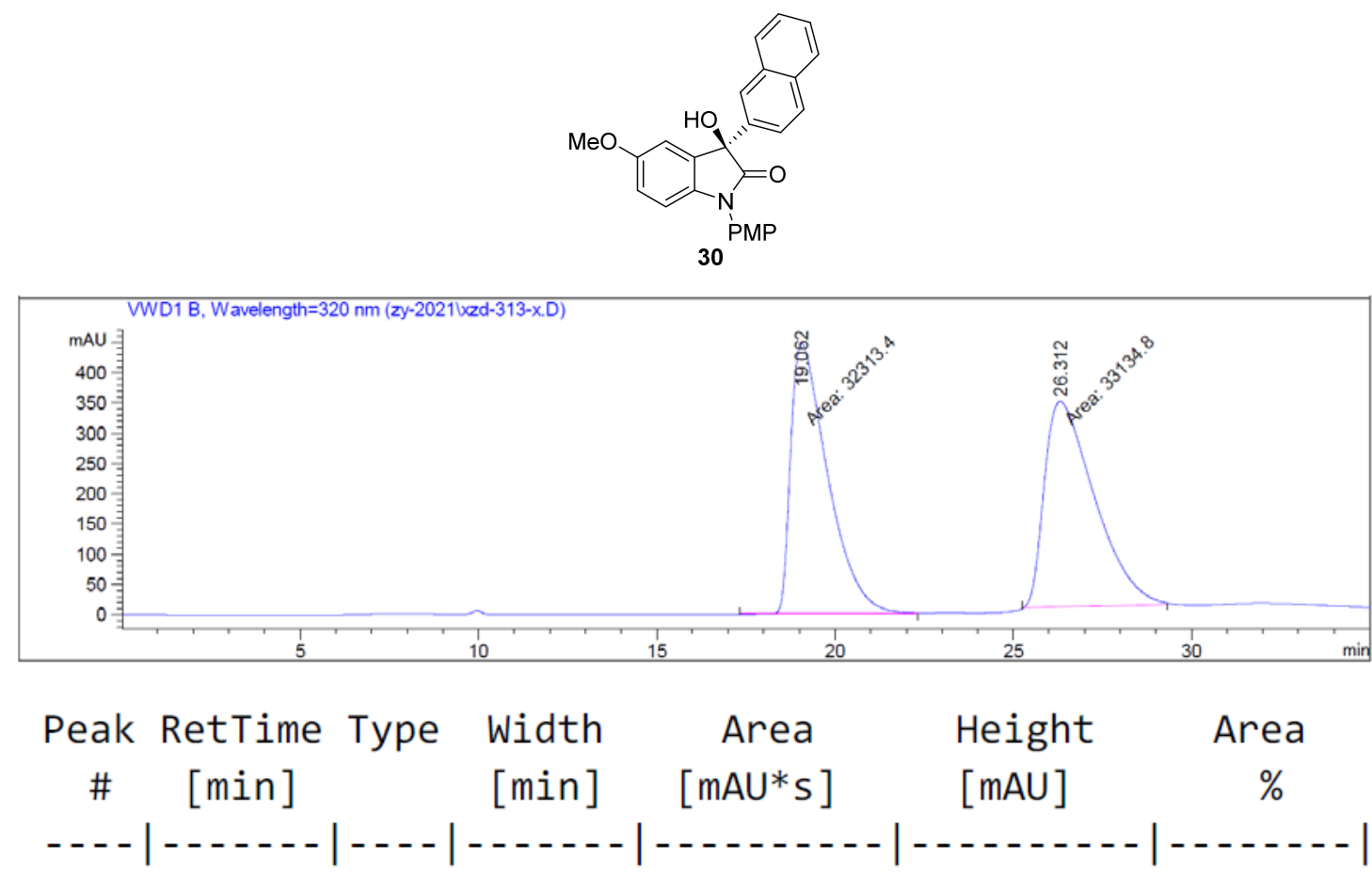

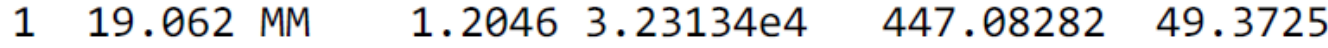

$2 \quad 26.312$ MM $\quad 1.6271 \quad 3.31348 \mathrm{e} 4 \quad 339.41116 \quad 50.6275$

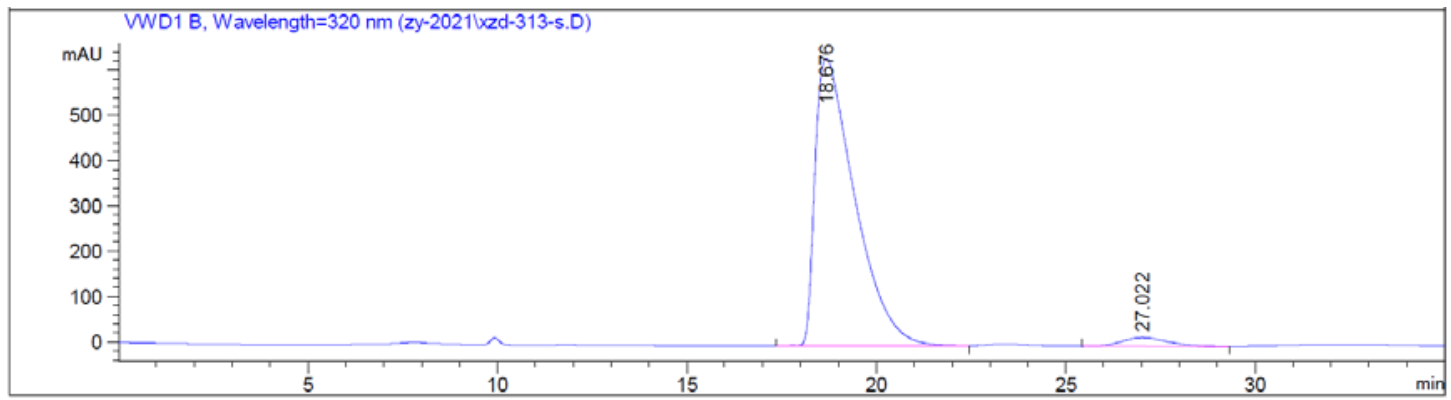

Peak RetTime Type Width Area Height Area

\# $\quad[\mathrm{min}] \quad[\mathrm{min}] \quad[\mathrm{mAU} * \mathrm{~s}] \quad[\mathrm{mAU}] \quad \%$

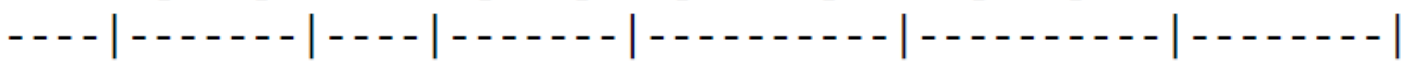

$\begin{array}{lllllll}1 & 18.676 & \text { VB R } & 1.0966 & 4.64012 \mathrm{e} 4 & 636.42285 & 96.7129\end{array}$

$2 \quad 27.022$ BB $\quad 1.3012 \quad 1577.10181 \quad 18.86840 \quad 3.2871$ 
HPLC Spectra of $\mathbf{3 1}$.
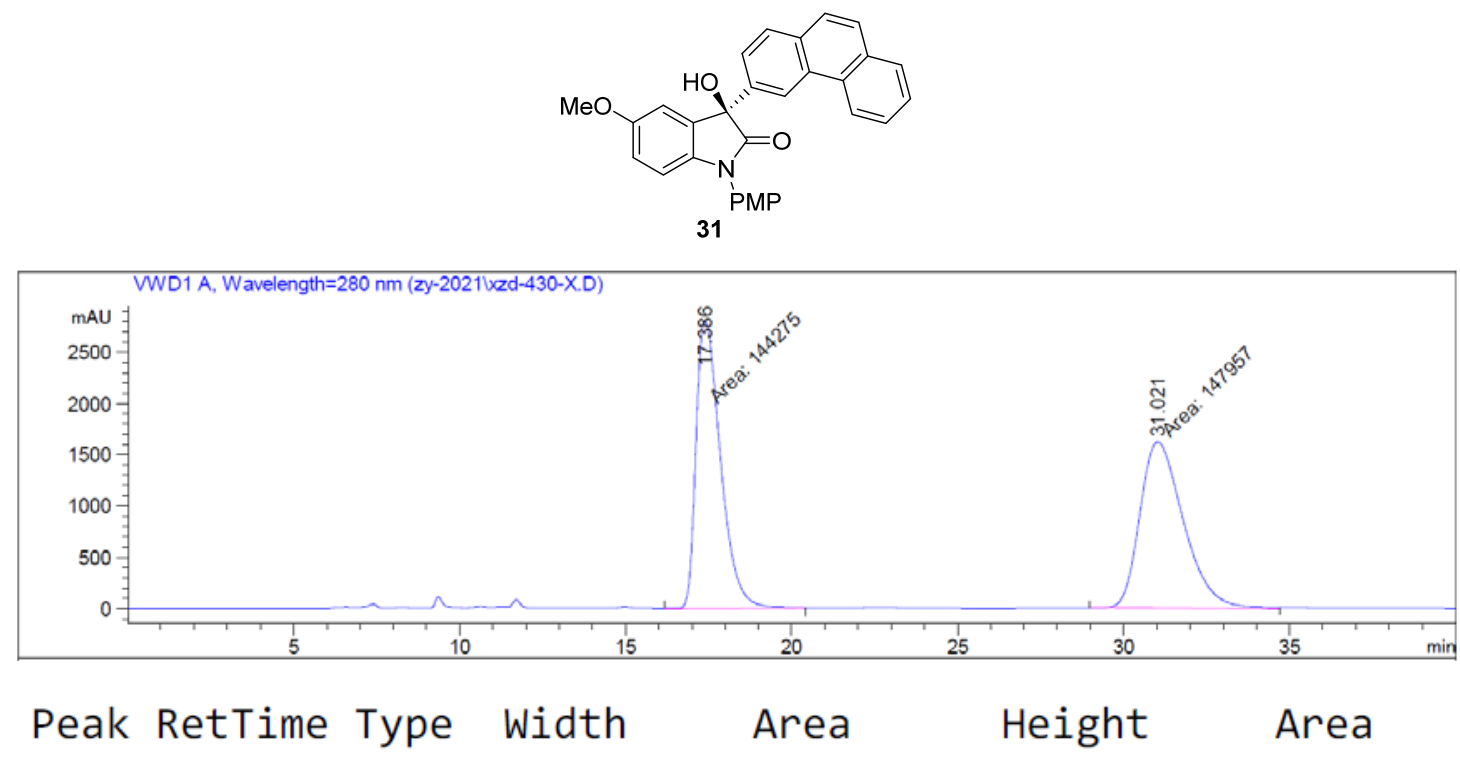

\# $[\mathrm{min}] \quad[\mathrm{min}] \quad[\mathrm{mAU} * \mathrm{~s}] \quad[\mathrm{mAU}] \quad \%$

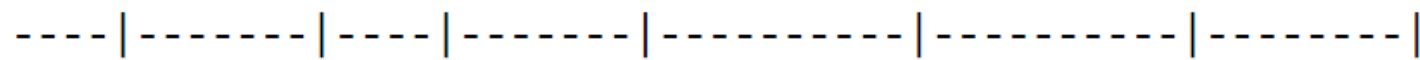

$\begin{array}{lllllll}1 & 17.386 \text { MM } & 0.8558 & 1.44275 \mathrm{e} 5 & 2809.78516 & 49.3700\end{array}$

$\begin{array}{llllll}2 & 31.021 \mathrm{MM} & 1.5233 & 1.47957 \mathrm{e} 5 & 1618.85950 & 50.6300\end{array}$

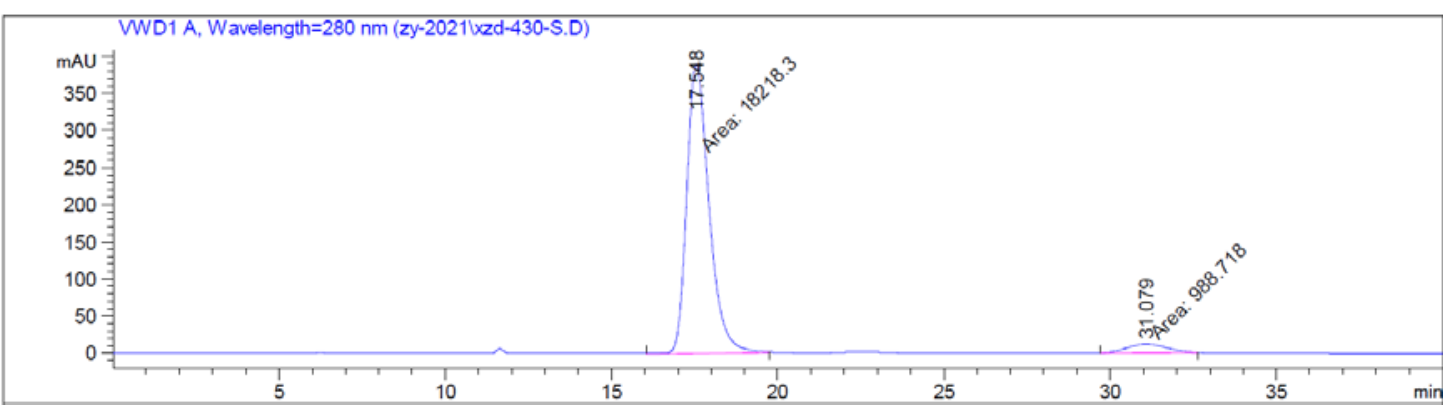

Peak RetTime Type Width Area Height Area

\# $[\mathrm{min}] \quad[\mathrm{min}] \quad[\mathrm{mAU} * \mathrm{~s}] \quad[\mathrm{mAU}] \quad \%$

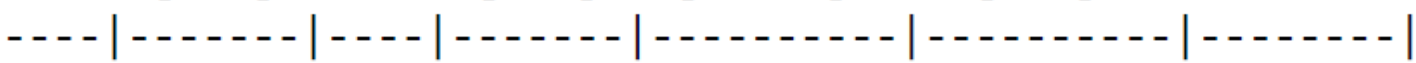

$\begin{array}{lllllll}1 & 17.548 \text { MM } & 0.7793 & 1.82183 \mathrm{e} 4 & 389.63379 & 94.8523\end{array}$

$\begin{array}{llllll}2 & 31.079 \text { MM } & 1.3782 & 988.71783 & 11.95646 & 5.1477\end{array}$ 
HPLC Spectra of 32.
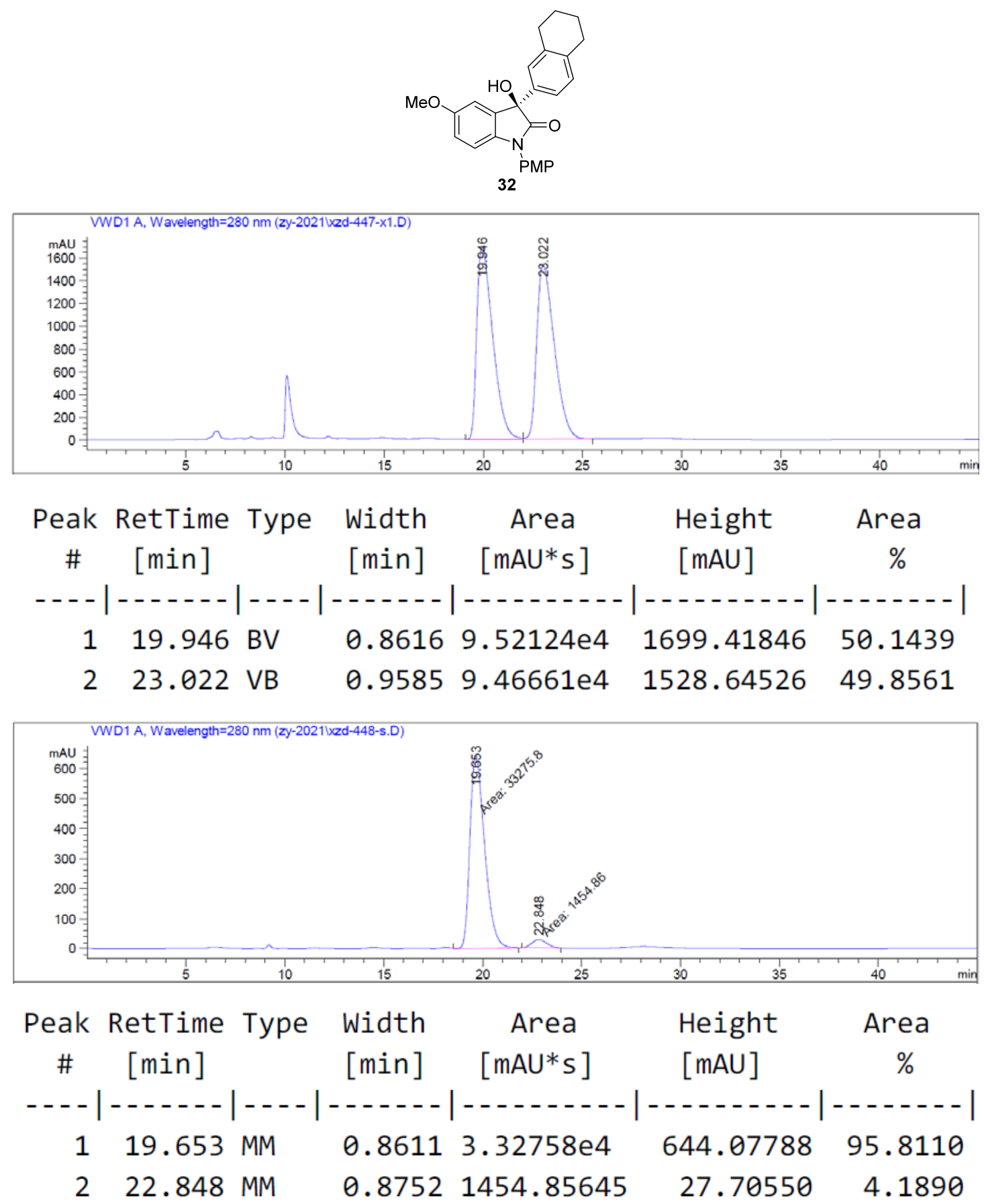
HPLC Spectra of $\mathbf{3 3}$.
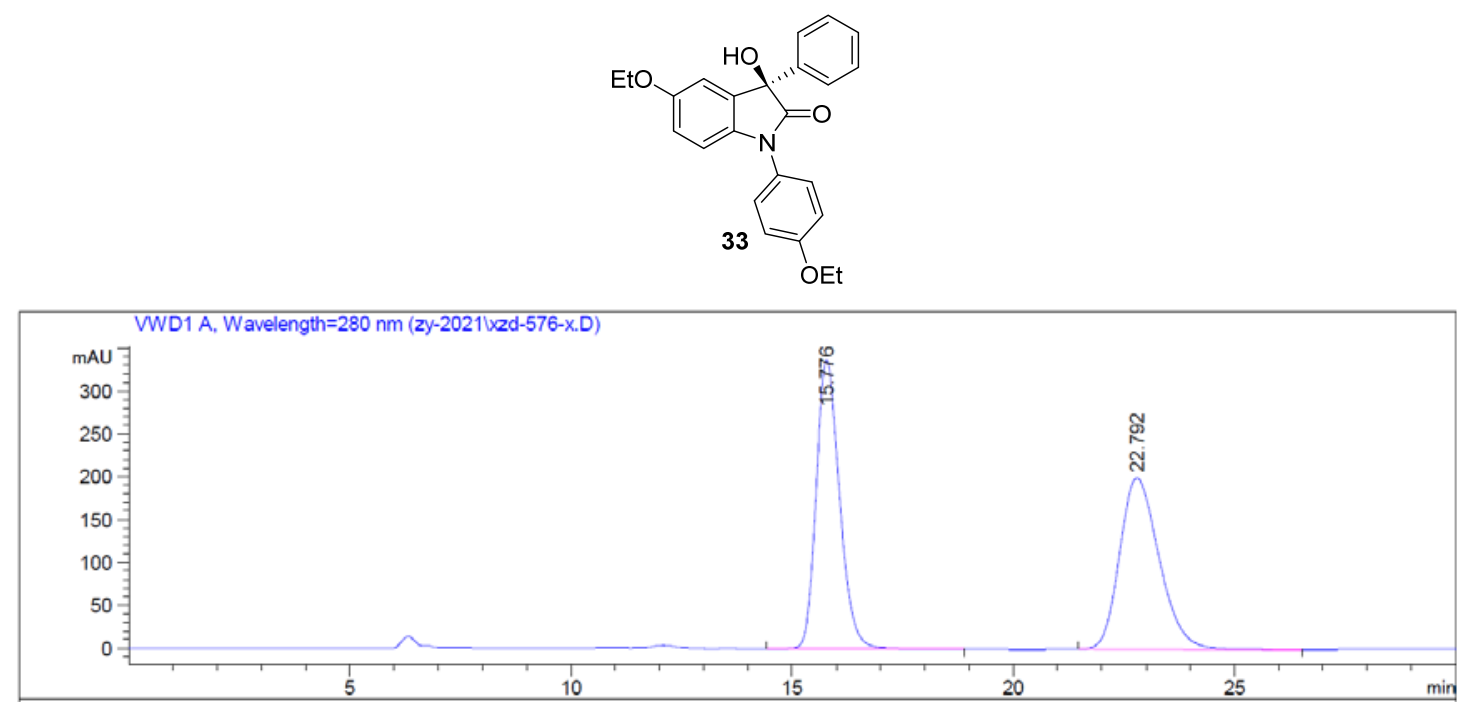

Peak RetTime Type Width Area Height Area

\# $[\mathrm{min}] \quad[\mathrm{min}] \quad[\mathrm{mAU} * \mathrm{~s}] \quad[\mathrm{mAU}] \quad \%$

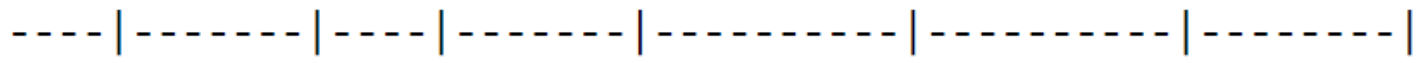

$\begin{array}{lllllll}1 & 15.776 & \text { BB } & 0.5671 & 1.23790 \mathrm{e} 4 & 336.56339 & 50.0261\end{array}$

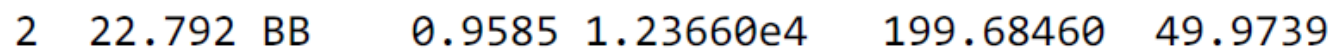

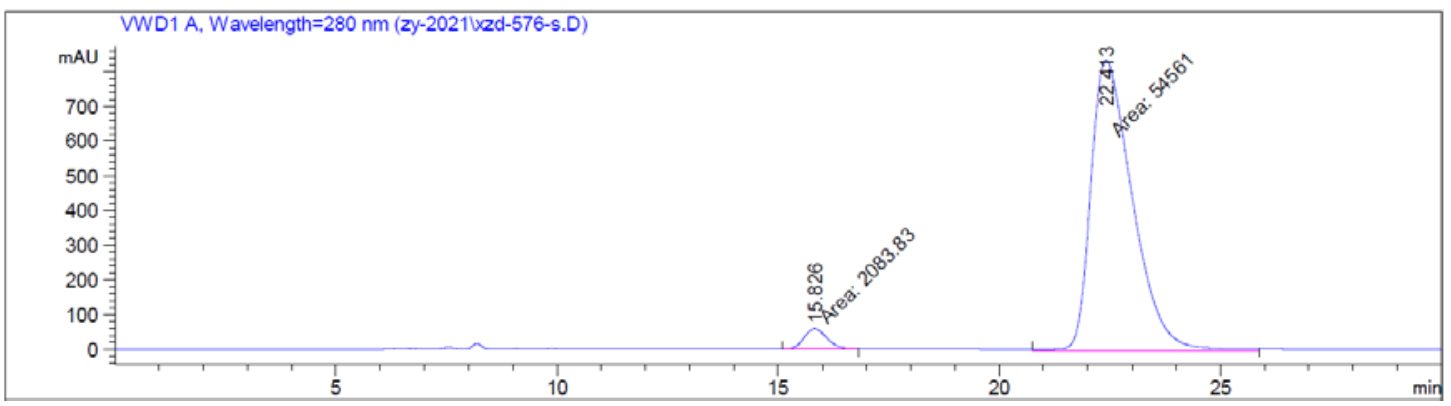

Peak RetTime Type Width Area Height Area

\# $[\mathrm{min}] \quad[\mathrm{min}] \quad[\mathrm{mAU} * \mathrm{~s}] \quad[\mathrm{mAU}] \quad \%$



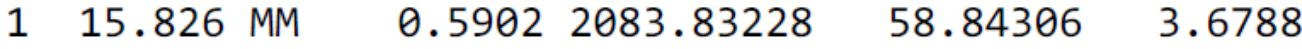

$\begin{array}{llllll}2 & 22.413 \text { MM } \quad 1.0891 & 5.45610 \mathrm{e} 4 & 834.93616 & 96.3212\end{array}$ 
HPLC Spectra of 34.
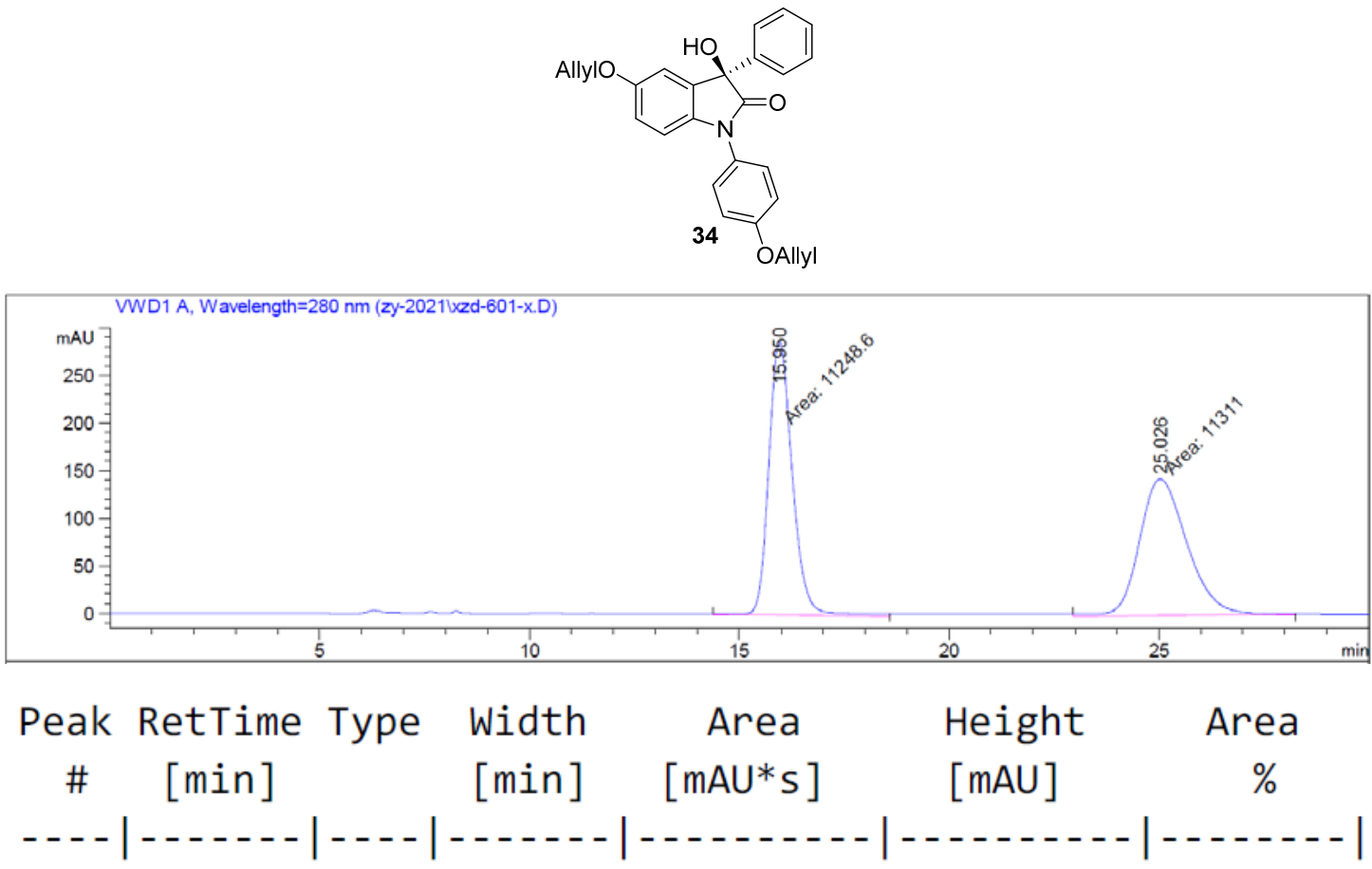

$\begin{array}{lllllll}1 & 15.950 \text { MM } & 0.6504 & 1.12486 \mathrm{e} 4 & 288.24744 & 49.8618\end{array}$

$\begin{array}{llllll}2 & 25.026 \text { MM } & 1.3138 & 1.13110 \mathrm{e} 4 & 143.48810 & 50.1382\end{array}$

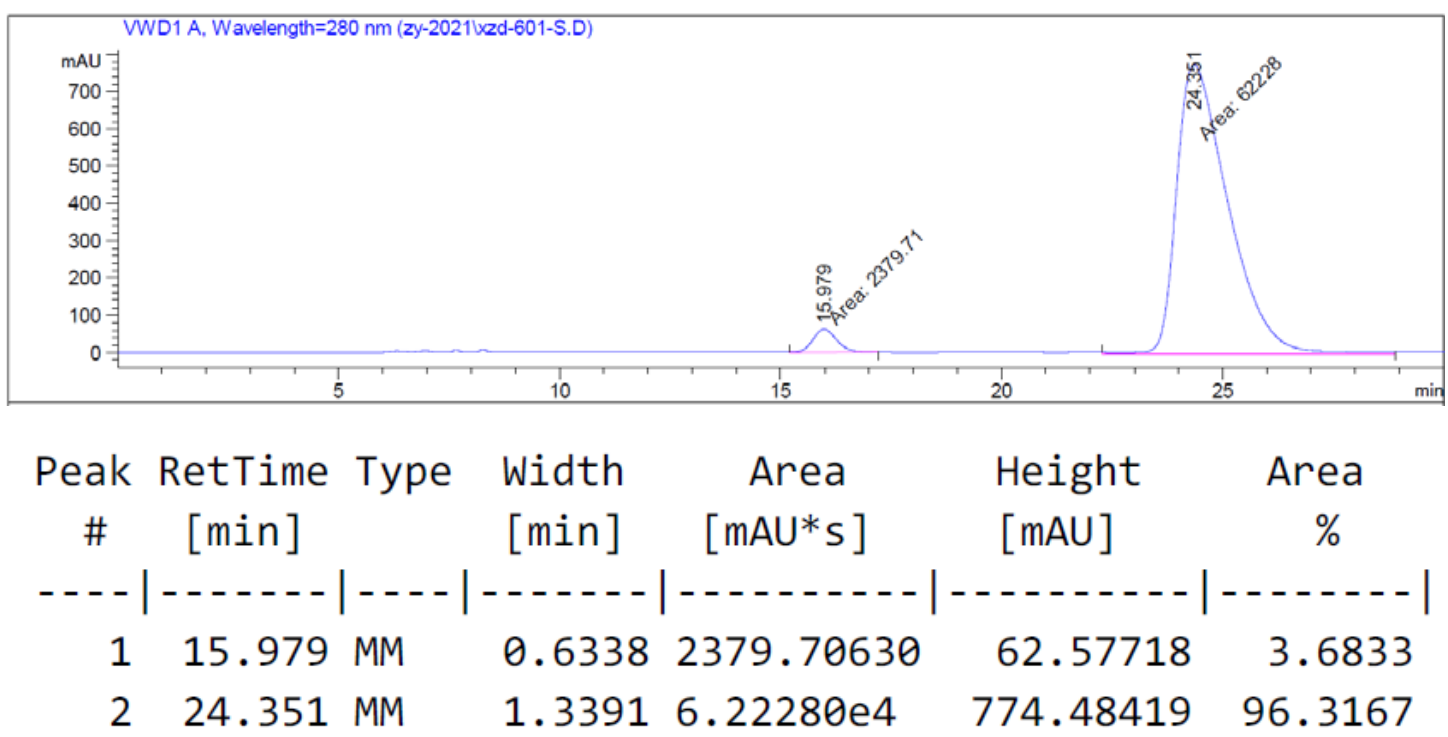


HPLC Spectra of 35.
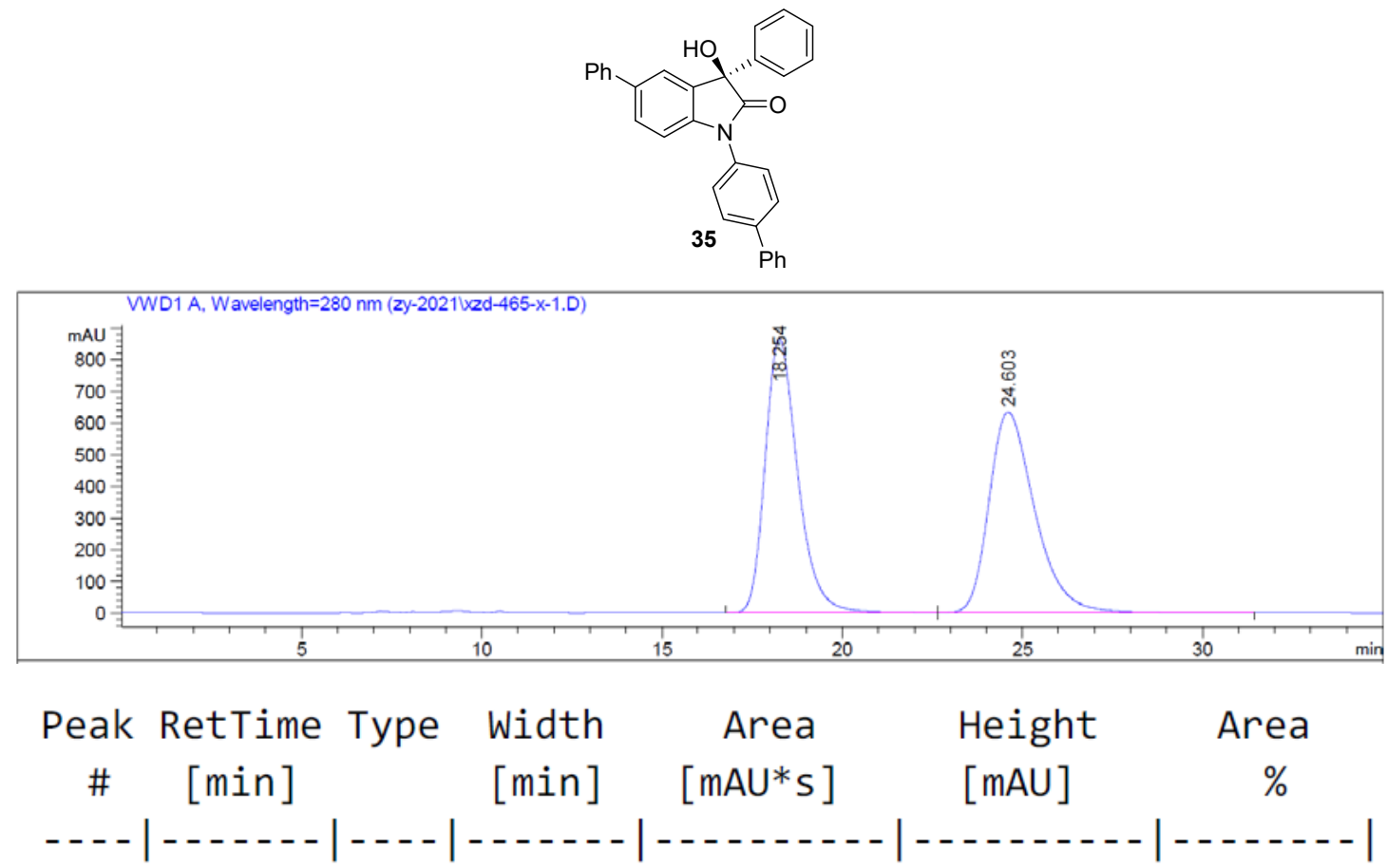

$\begin{array}{lllllll}1 & 18.254 & \text { BB } & 0.9581 & 5.41632 \mathrm{e} 4 & 865.46484 & 49.2367\end{array}$

$2 \quad 24.603$ BB $\quad 1.34625 .58425 \mathrm{e} 4 \quad 633.63342 \quad 50.7633$

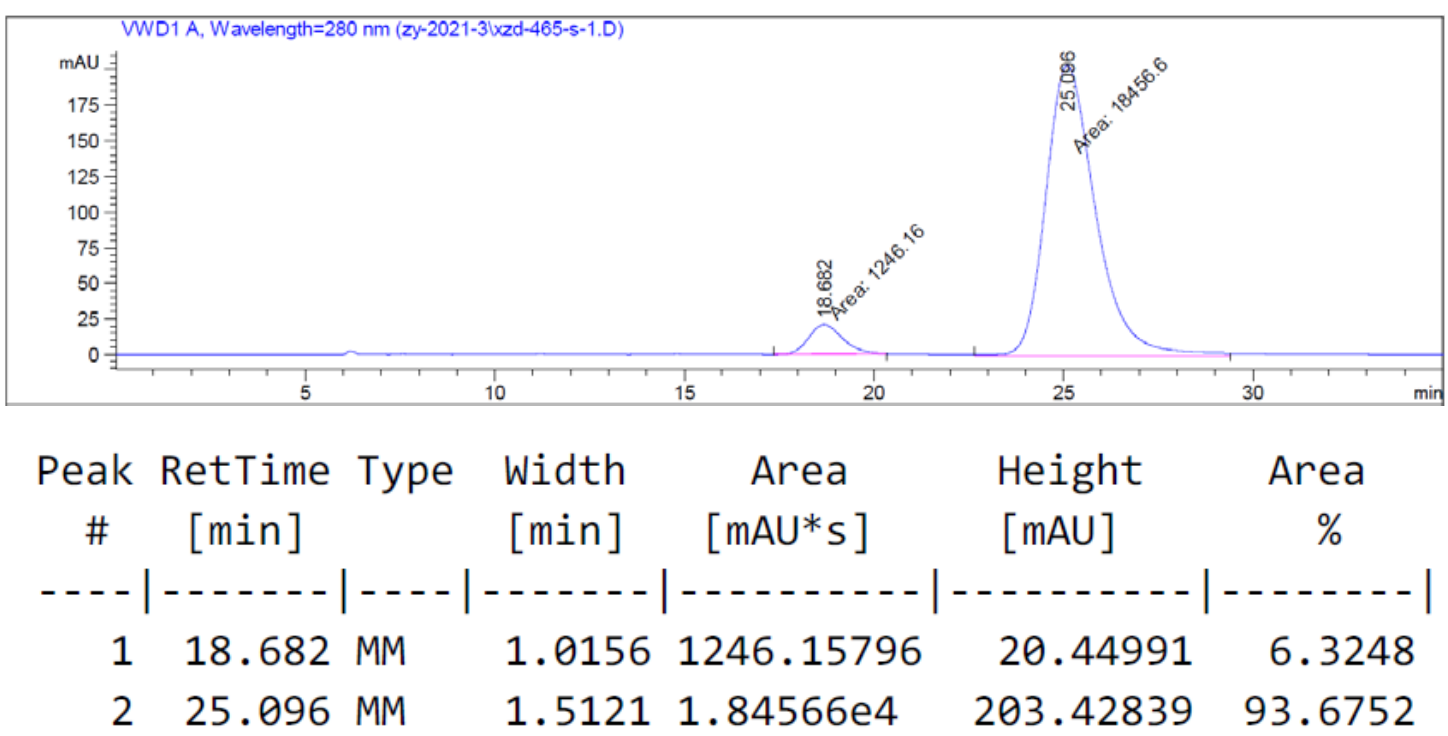


HPLC Spectra of $\mathbf{3 6}$.
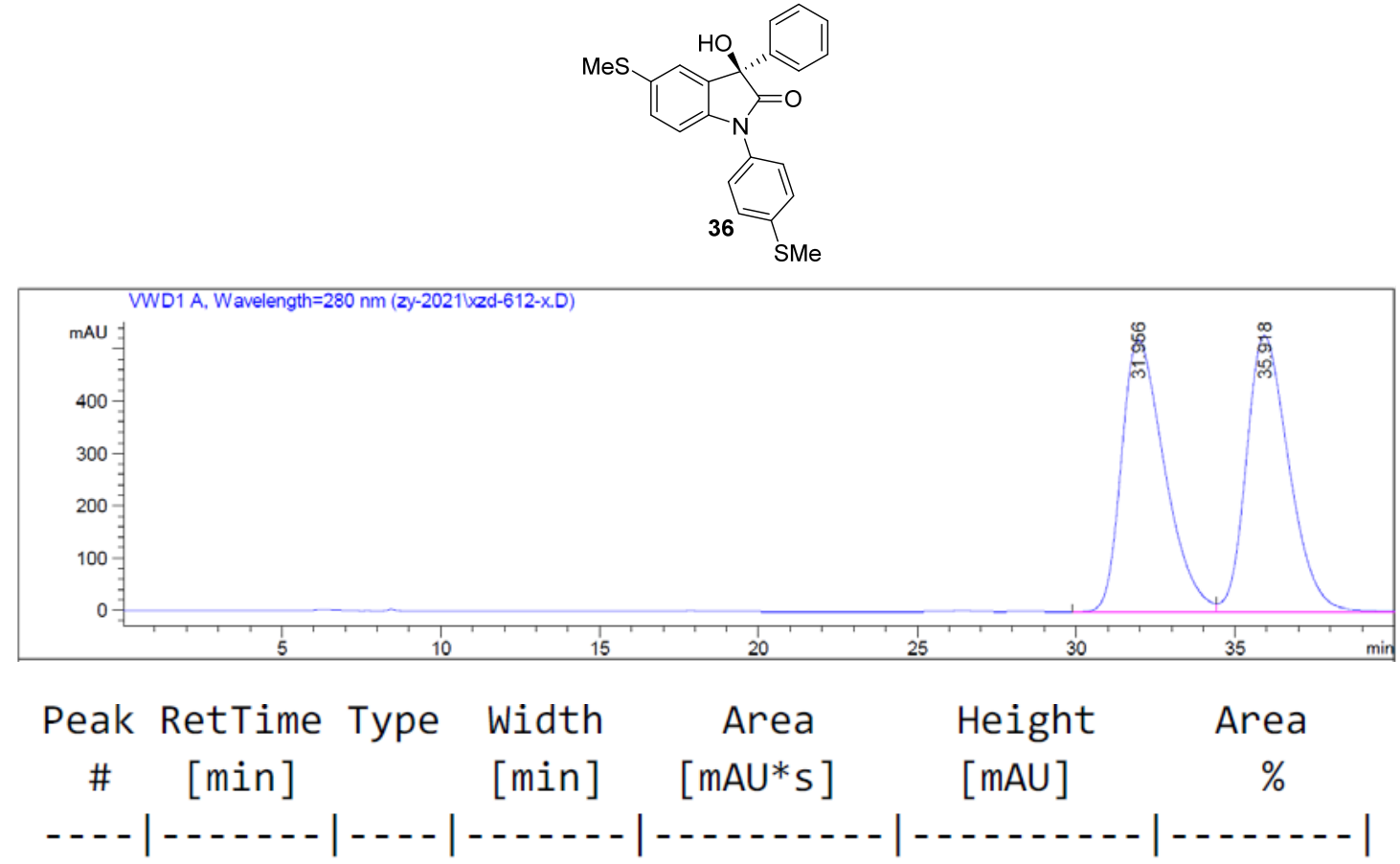

$\begin{array}{lllllll}1 & 31.956 & \text { BV } & 1.4418 & 4.89696 \mathrm{e} 4 & 519.45032 & 49.8124\end{array}$

$2 \quad 35.918$ VB $\quad 1.4381 \quad 4.93385 \mathrm{e} 4 \quad 528.03131 \quad 50.1876$

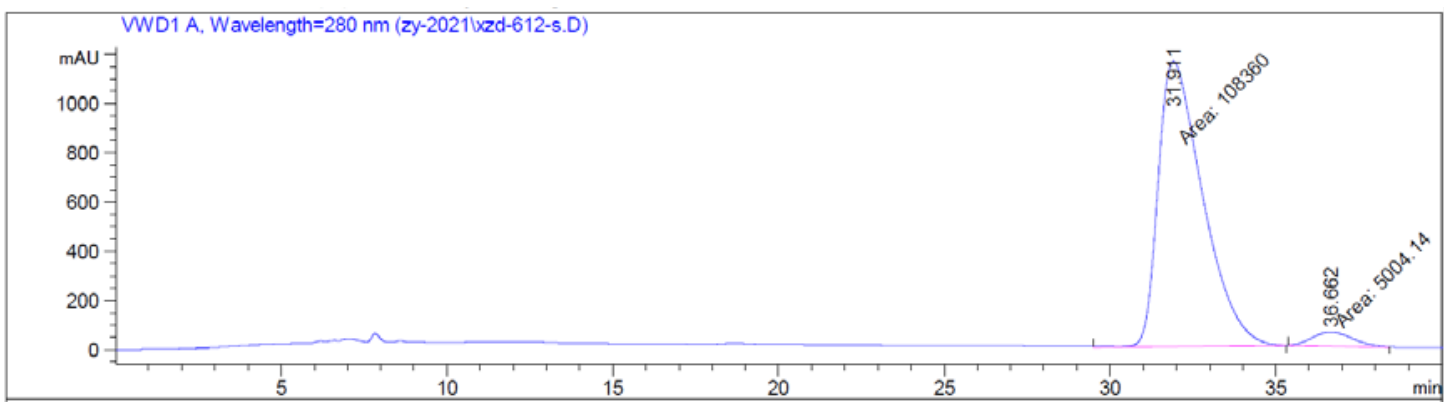

Peak RetTime Type Width Area Height Area

$\left.\begin{array}{llll}\# & {[\mathrm{~min}]} & {[\mathrm{min}]} & {[\mathrm{mAU} * \mathrm{~s}]}\end{array}\right]$

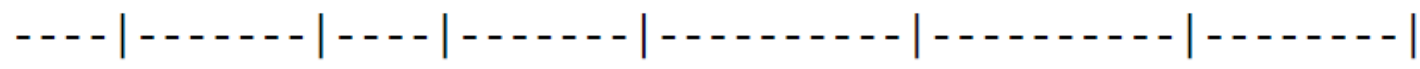

$\begin{array}{lllllll}1 & 31.911 \mathrm{MM} & 1.5576 & 1.08360 \mathrm{e} 5 & 1159.49658 & 95.5858\end{array}$

$\begin{array}{llllll}2 & 36.662 \text { MM } & 1.4254 & 5004.14404 & 58.51008 & 4.4142\end{array}$ 
HPLC Spectra of 37.
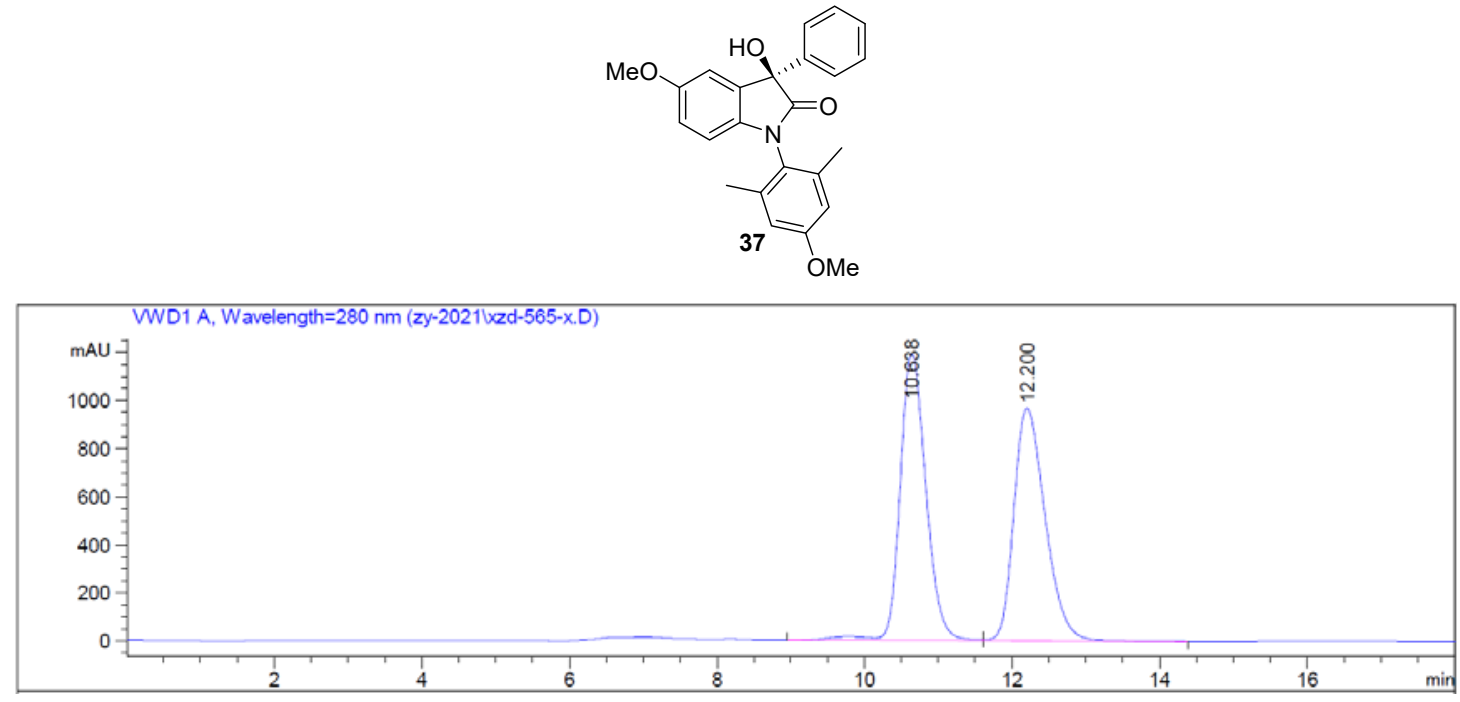

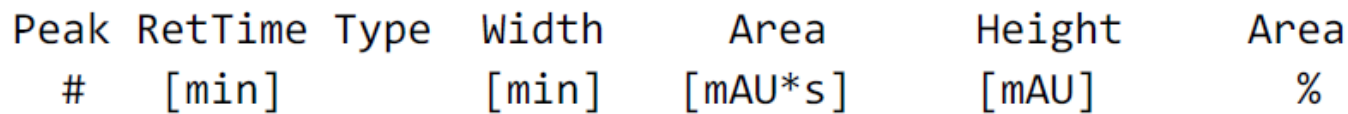

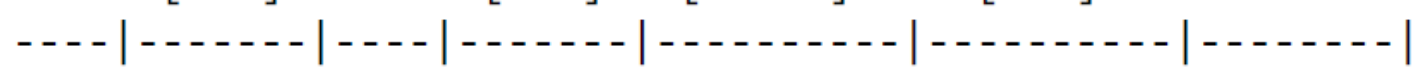

$\begin{array}{lllllll}1 & 10.638 & \text { VV R } & 0.3710 & 2.91901 \mathrm{e} 4 & 1194.80591 & 50.5581\end{array}$

$\begin{array}{lllllll}2 & 12.200 & V B & 0.4578 & 2.85457 e 4 & 968.70020 & 49.4419\end{array}$

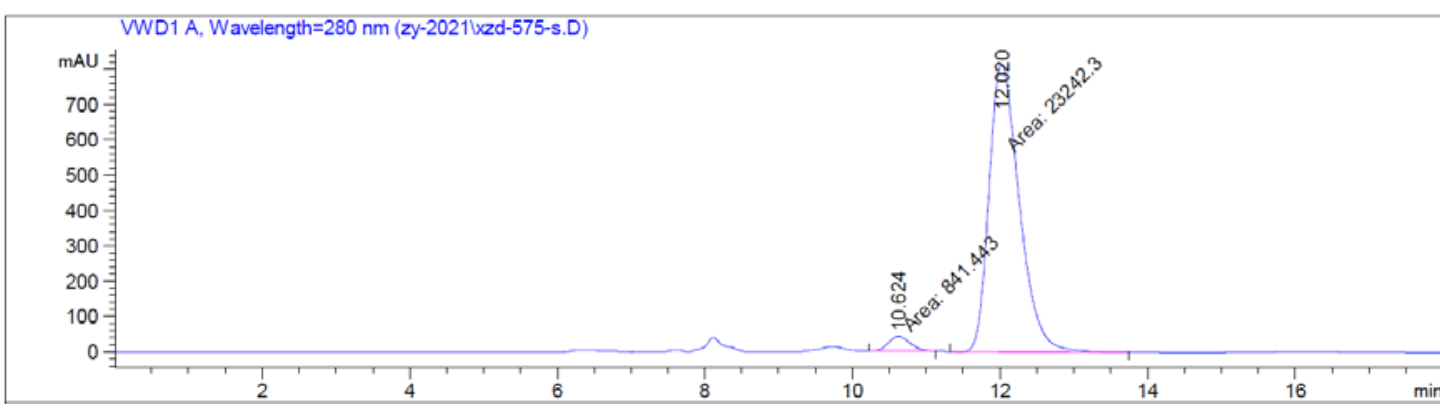

Peak RetTime Type Width Area Height Area

\# $\quad[\mathrm{min}] \quad[\mathrm{min}] \quad[\mathrm{mAU} * \mathrm{~s}] \quad[\mathrm{mAU}] \quad \%$

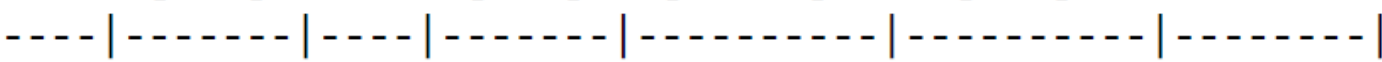

$\begin{array}{lllllll}1 & 10.624 & \text { MM } & 0.3354 & 841.44287 & 41.81080 & 3.4938\end{array}$

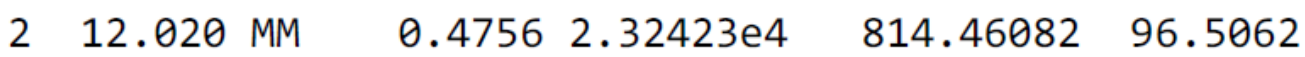


HPLC Spectra of $\mathbf{3 8}$.
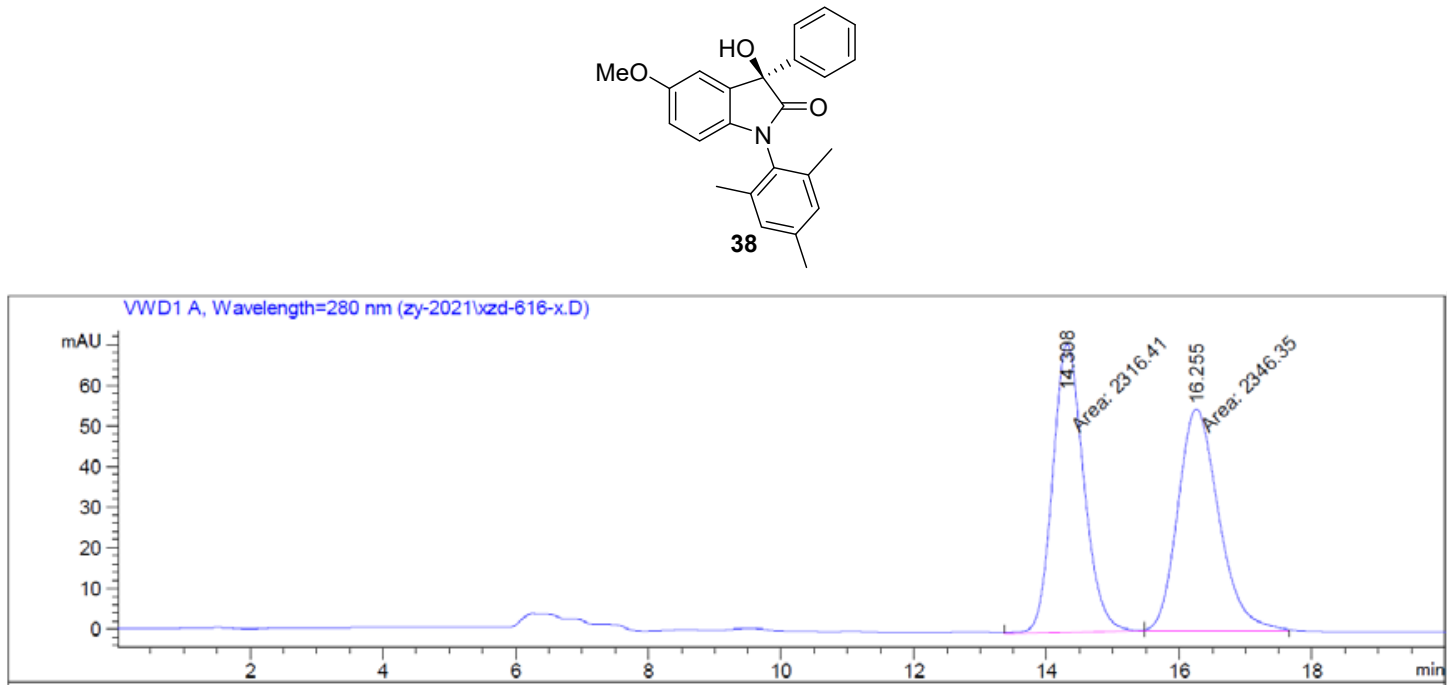

Peak RetTime Type Width Area Height Area

\# $[\mathrm{min}] \quad[\mathrm{min}] \quad[\mathrm{mAU} * \mathrm{~s}] \quad[\mathrm{mAU}] \quad \%$

- - - - | - - - - - | - - - | - - - - - - | - - - - - - - | - - - - - - - | - - - - - - |
114.308 MM
$\begin{array}{llll}0.5444 & 2316.40845 & 70.91544 & 49.6790\end{array}$

216.255 MM

$\begin{array}{llll}0.7166 & 2346.34644 \quad 54.57200 & 50.3210\end{array}$

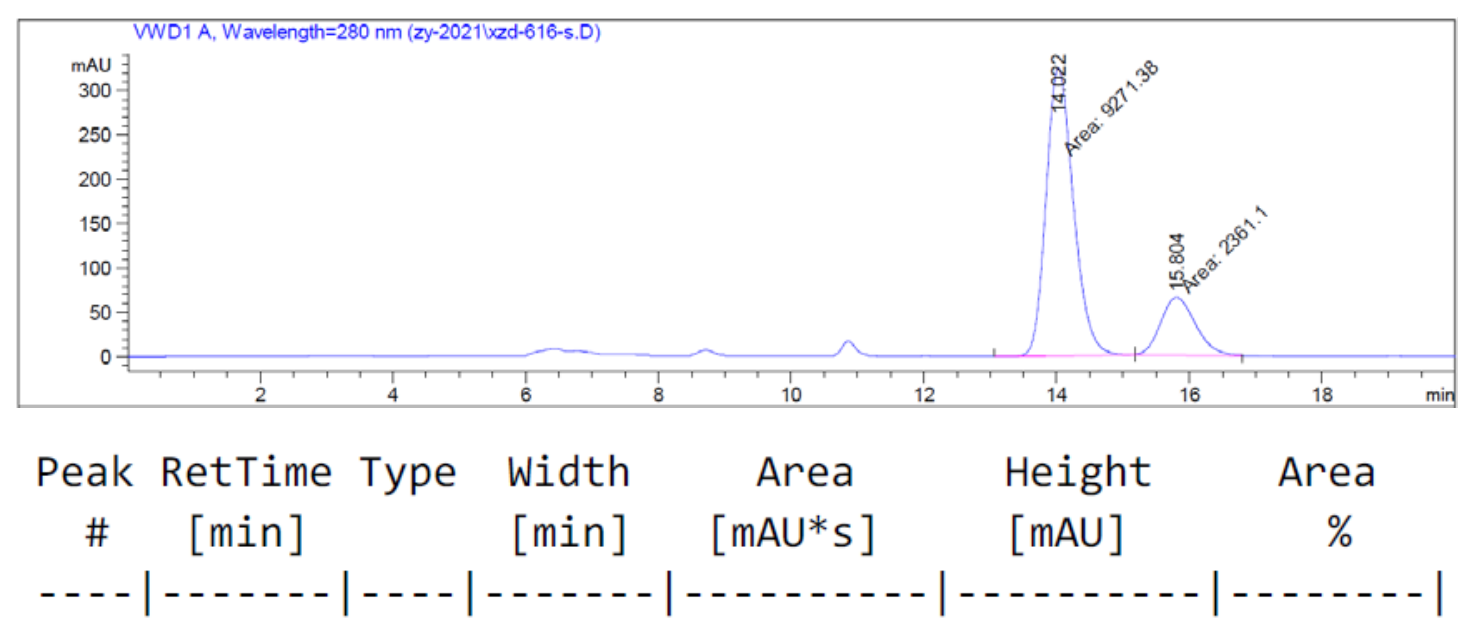

$\begin{array}{lllllll}1 & 14.022 & \text { MM } & 0.4768 & 9271.37988 & 324.05276 & 79.7025\end{array}$

$\begin{array}{llllll}2 & 15.804 \text { MM } \quad 0.6047 & 2361.09692 & 65.07349 & 20.2975\end{array}$ 
HPLC Spectra of $\mathbf{4 1 .}$
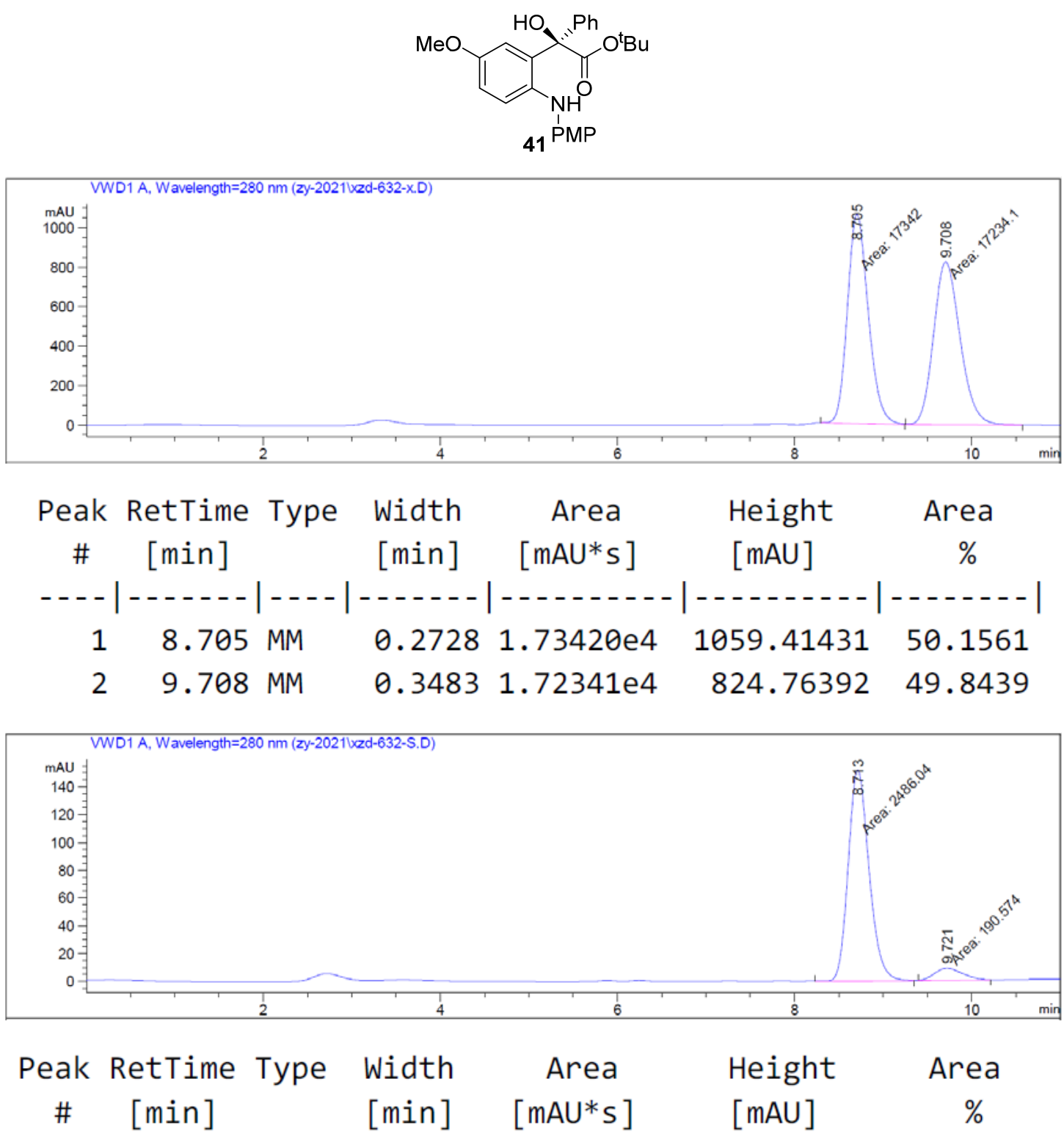

\begin{tabular}{rrr|rrr|r}
1 & 8.713 & MM & 0.2722 & 2486.04272 & 152.23254 & 92.8801 \\
2 & 9.721 & MM & 0.3655 & 190.57370 & 8.69108 & 7.1199
\end{tabular}


HPLC Spectra of Scale-up Experiment.
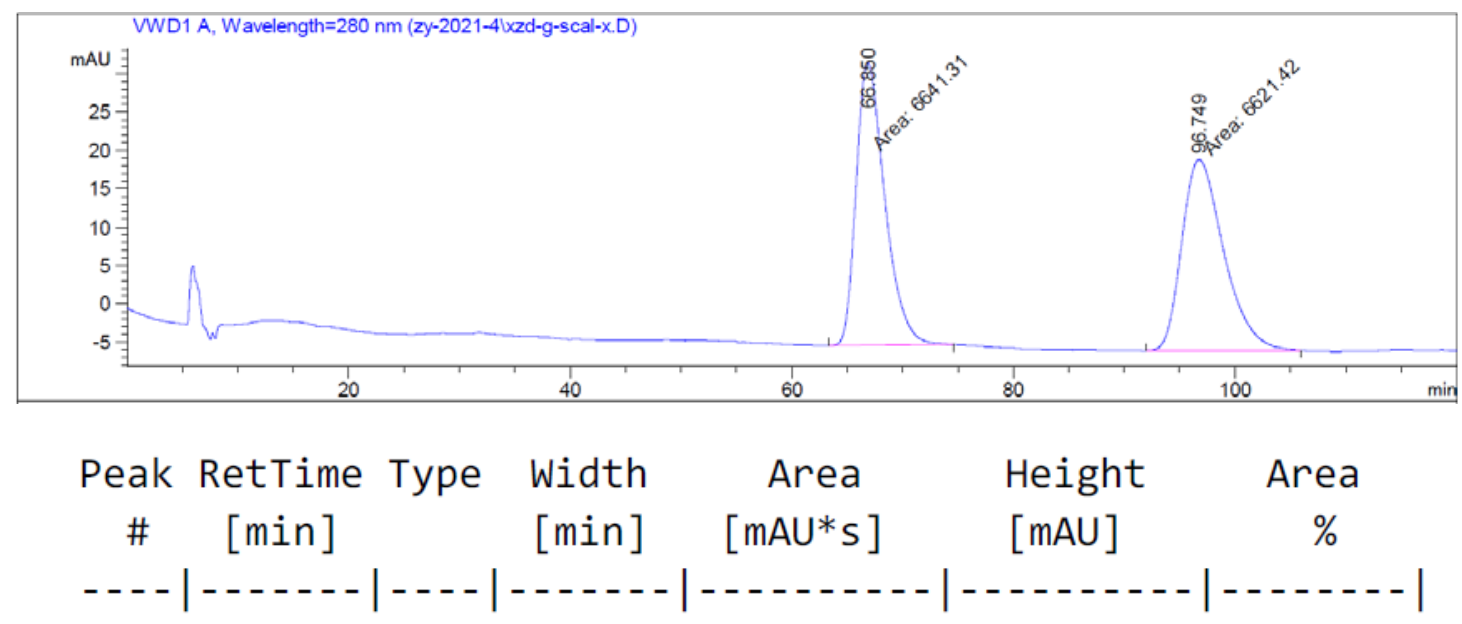
166.850 MM
3.00046641 .31445
$36.89099 \quad 50.0750$
296.749 MM
4.41426621 .42139
$25.00068 \quad 49.9250$

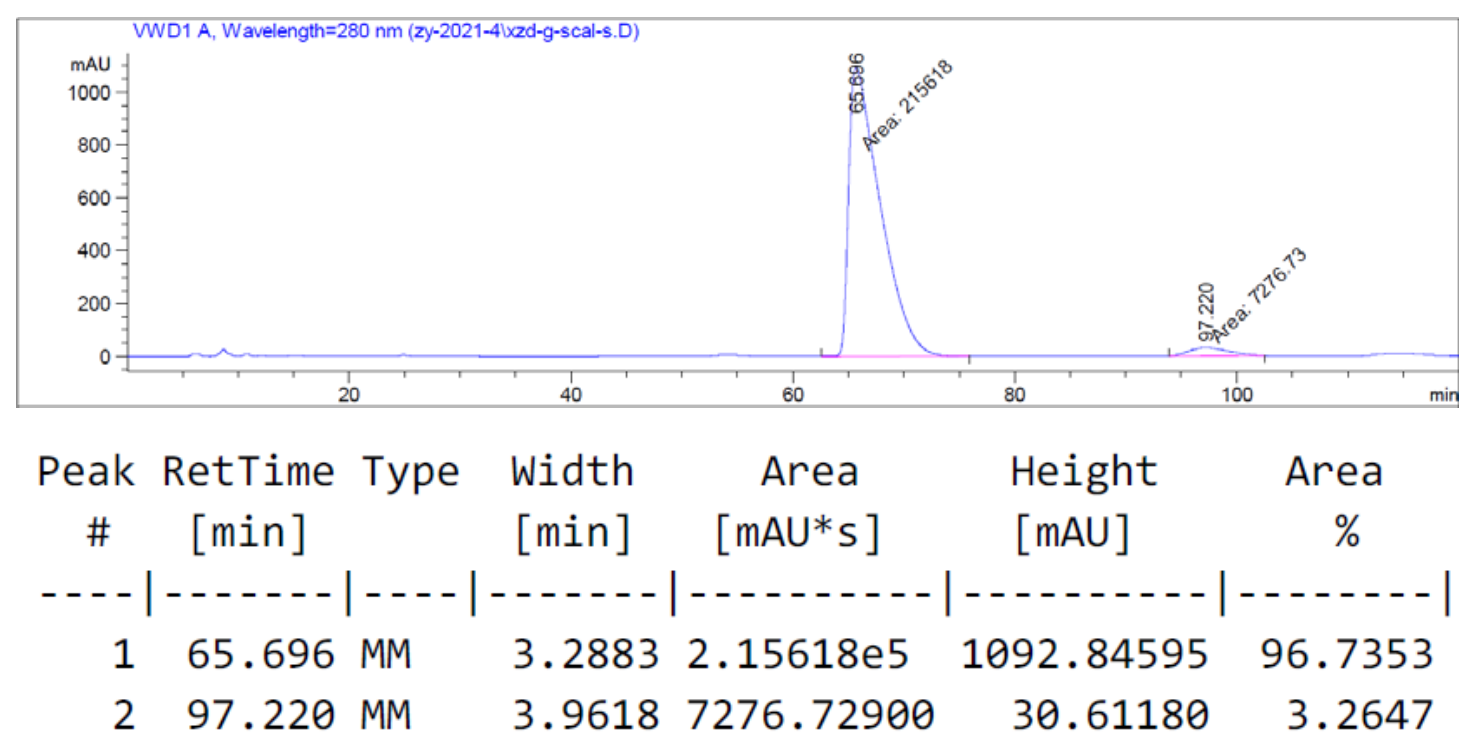

